Prepared in cooperation with the Bureau of Land Management,

Montana Department of Environmental Quality,

Montana Department of Fish, Wildlife, and Parks,

U.S. Environmental Protection Agency,

Wyoming Department of Environmental Quality, and

Wyoming Game and Fish Department

Assessment of Ecological Conditions and Potential Effects of Water Produced from Coalbed Natural Gas Development on Biological Communities in Streams of the Powder River Structural Basin, Wyoming and Montana, 2005-08

Scientific Investigations Report 2010-5124 
FRONT COVER AND BOTTOM RIGHT ON BACK COVER:

Powder River below Burger Draw, Wyo. (site P4), July 19, 2010.

Photograph by Gregory K. Boughton, U.S. Geological Survey.

BACK COVER, LEFT HALF AND MIDDLE RIGHT:

Powder River near Arvada, Wyo., July 22, 2010.

Photograph by David A. Peterson, U.S. Geological Survey.

Note: The location of Arvada is shown in figure 1. This photograph was not an established sampling site during 2005-08, and is located between sites P8 and P9.

BACK COVER, TOP RIGHT:

Little Powder River above Dry Creek near Weston, Wyo. (site P15), July 6, 2010.

Photograph by Gregory K. Boughton, U.S. Geological Survey. 


\section{Assessment of Ecological Conditions and Potential Effects of Water Produced from Coalbed Natural Gas Development on Biological Communities in Streams of the Powder River Stuctural Basin, Wyoming and Montana, 2005-08}

By David A. Peterson, Melanie L. Clark, Katharine Foster, Peter R. Wright, and Gregory K. Boughton

Prepared in cooperation with the Bureau of Land Management,

Montana Department of Environmental Quality,

Montana Department of Fish, Wildlife, and Parks, U.S. Environmental Protection Agency,

Wyoming Department of Environmental Quality, and

Wyoming Game and Fish Department

Scientific Investigations Report 2010-5124 


\section{U.S. Department of the Interior \\ KEN SALAZAR, Secretary \\ U.S. Geological Survey \\ Marcia K. McNutt, Director}

\section{U.S. Geological Survey, Reston, Virginia: 2010}

For more information on the USGS - the Federal source for science about the Earth, its natural and living resources, natural hazards, and the environment, visit http://www.usgs.gov or call 1-888-ASK-USGS

For an overview of USGS information products, including maps, imagery, and publications, visit http://www.usgs.gov/pubprod

To order this and other USGS information products, visit http://store.usgs.gov

Any use of trade, product, or firm names is for descriptive purposes only and does not imply endorsement by the U.S. Government.

Although this report is in the public domain, permission must be secured from the individual copyright owners to reproduce any copyrighted materials contained within this report.

Suggested citation:

Peterson, D.A., Clark, M.L., Foster, Katharine, Wright, P.R., and Boughton, G.K., 2010, Assessment of ecological conditions and potential effects of water produced from coalbed natural gas development on biological communities in streams of the Powder River structural basin, Wyoming and Montana, 2005-08: U.S. Geological Survey Scientific Investigations Report 2010-5124, 84 p. 


\section{Contents}

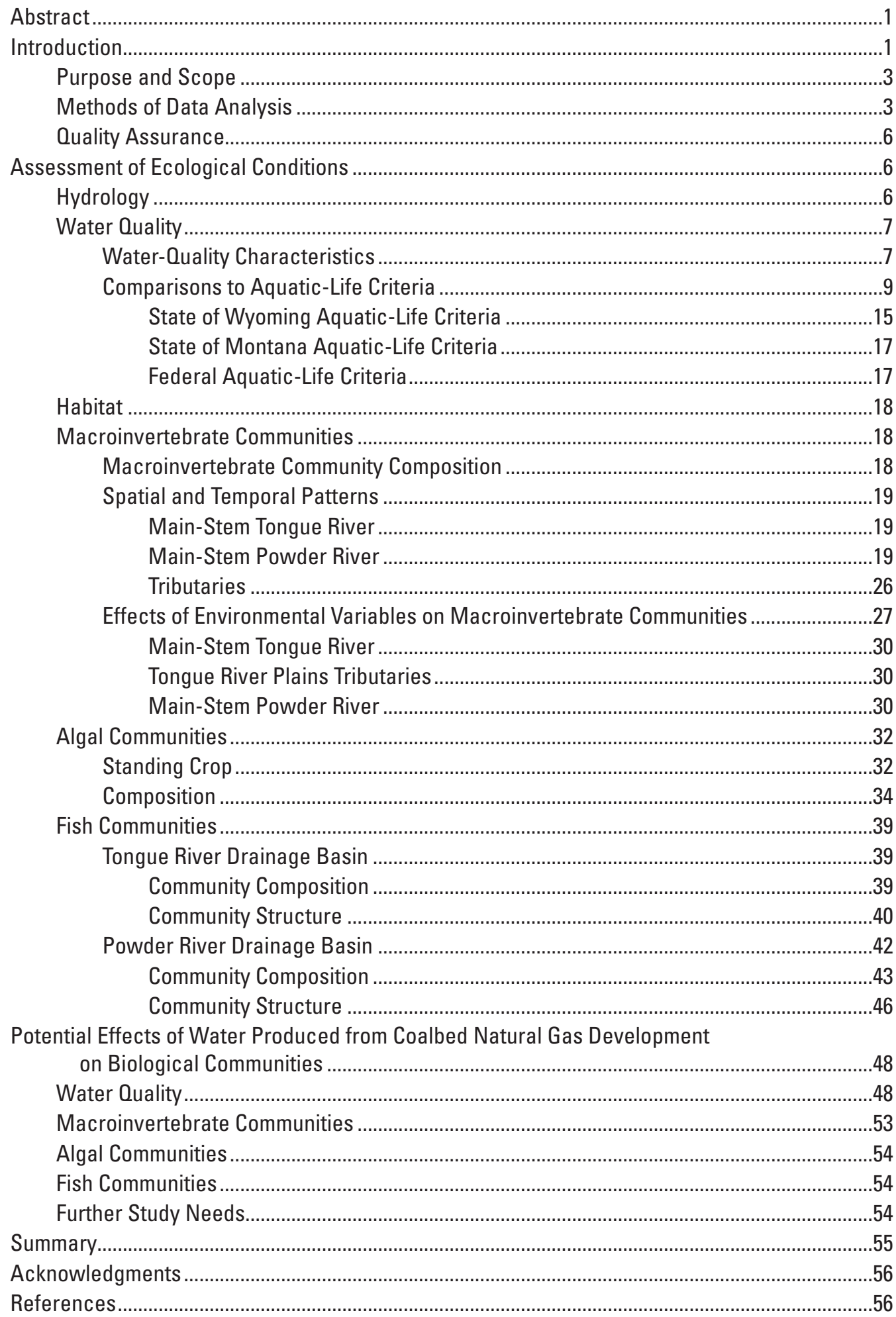

Appendix. Summary Statistics for Water-Quality Constituents, Powder River Structural

Basin, Wyoming and Montana, Water Years 2005-08. 


\section{Figures}

1. Location of aquatic ecology sampling sites in the Powder River structural basin, Wyoming and Montana, 2005-08

2. Cumulative water production as of September 30,2008 , from coalbed natural gas wells in the Powder River structural basin, Wyoming and Montana

3. Streamflow at the time of ecological sampling - Mean streamflow values from 2005 to 2008 for sites on $(A)$ main-stem Tongue River, and $(B)$ mainstem Powder River; instantaneous streamflow values for selected sites on (C) main-stem Tongue River, and (D) main-stem Powder River, Wyoming, and Montana

4. Mean values for specific conductance, dissolved sodium, and alkalinity for the Tongue River and Powder River during ecological sampling events, Wyoming and Montana, 2005-08

5. Similarities of macroinvertebrate community taxonomic composition within stream groups depicted by nonmetric multidimensional scaling ordinations, Powder River structural basin, Wyoming and Montana, 2007-08.

6. Selected macroinvertebrate community metrics by stream group, Powder River structural basin, Wyoming and Montana, 2005-08.

7. Mean values for selected macroinvertebrate community metrics by site and by year, main-stem Tongue River, Wyoming and Montana, 2005-08

8. Mean values for selected macroinvertebrate metrics by site and by year, main-stem Powder River, Wyoming and Montana, 2005-08

9. Principal components ordination showing relations among sampling sites based on selected environmental variables, Powder River structural basin, Wyoming and Montana, 2007

10. Selected environmental variables correlated with macroinvertebrate communities, Powder River structural basin, 2005-08

11. Similarities of algal community taxonomic composition within drainage basins and within habitats, depicted by nonmetric multidimensional scaling ordination, Powder River structural basin, Wyoming and Montana, 2007.

12. Algal community composition by drainage basin and habitat, Powder River structural basin, 2007

13. Relative abundance of fish by species in samples from the Tongue River drainage basin, Wyoming and Montana, 2005-06 and 2008

14. Selected unadjusted fish community metric values for the main-stem Tongue River, Wyoming and Montana, 2005-06 and 2008

15. Index of Biotic Integrity scores (IBI) for fish communities of the Tongue River tributaries, Wyoming and Montana, 2005-06 and 2008

16. Relative abundance of fish by species in samples from the Powder River drainage basin, Wyoming and Montana, 2004-06 and 2008.

17. Selected unadjusted fish community metric values for the main-stem

Powder River, Wyoming and Montana, 2004-06 and 2008.

18. Index of Biotic Integrity (IBI) scores for fish communities in selected Powder River tributaries, Wyoming, 2005-06 and 2008. 


\section{Tables}

1. Ecological sampling sites on streams in the Powder River structural basin, Wyoming and Montana, 2005-08

2. Biological quality-control samples, Powder River structural basin, Wyoming and Montana, 2005-08

3. Environmental variables associated with ecological samples, Powder River structural basin, Wyoming and Montana, 2005-08.

4. State and Federal criteria for the protection of aquatic life

5. Aquatic-life criteria comparison for water samples collected from study sites in the Powder River structural basin, Wyoming and Montana, water years 2005-08

6. Macroinvertebrate community metrics for streams in the Powder River structural basin, Wyoming and Montana, 2005-08

7. Analysis of variance in macroinvertebrate community metrics, main-stem Tongue River and main-stem Powder River, Wyoming and Montana, 2005-08

8. Environmental variables best correlated with macroinvertebrate community taxonomic composition, by stream group and year, Powder River structural basin, Wyoming and Montana, 2005-08

9. Correlation coefficients of macroinvertebrate community metrics with environmental variables, main-stem Powder River, Wyoming and Montana, 2005-08

10. Algal chlorophyll-a concentration, ash-free dry mass concentration, density, and biovolume, Powder River structural basin, Wyoming and Montana, 2005 and 2007.

11. Algal community metrics for streams in the Powder River structural basin, Wyoming and Montana, 2005 and 2007

12. Fish abundance in samples from the Tongue River drainage basin, Wyoming and Montana, 2005-06 and 2008

13. Ecological characteristics of fish species sampled in the Powder River Structural Basin, Wyoming and Montana, 2005-08.

14. Unadjusted fish community metric values for the main-stem Tongue River, Wyoming and Montana, 2005-06 and 2008

15. Analysis of variance in fish community metrics, main-stem Tongue River and main-stem Powder River, Wyoming and Montana, 2005-08

16. Fish community metric and Index of Biotic Integrity (IBI) scores for the Tongue and Powder River tributaries, Wyoming and Montana, 2005-06 and 2008

17. Fish abundance in samples from Powder River drainage basin, Wyoming and Montana, 2004-06 and 2008

18. Unadjusted fish community metric values for the main-stem Powder River, Wyoming and Montana, 2004-06 and 2008 


\section{Conversion Factors and Datums}

\begin{tabular}{lcl}
\hline Multiply & By & To obtain \\
\hline inch (in.) & 2.54 & centimeter $(\mathrm{cm})$ \\
foot $(\mathrm{ft})$ & 0.3048 & meter $(\mathrm{m})$ \\
mile $(\mathrm{mi})$ & 1.609 & kilometer $(\mathrm{km})$ \\
acre-foot (acre-ft) & 1,233 & cubic meter $\left(\mathrm{m}^{3}\right)$ \\
cubic foot per second $(\mathrm{ft} / \mathrm{s})$ & 0.02832 & cubic meter per second $\left(\mathrm{m}^{3} / \mathrm{s}\right)$ \\
micrometer $(\mu \mathrm{m})$ & 0.00003937 & inch $(\mathrm{in})$. \\
millimeter $(\mathrm{mm})$ & 0.03937 & inch $(\mathrm{in})$. \\
cubic centimeter per square meter $\left(\mathrm{cm}^{3} / \mathrm{m}^{2}\right)$ & 0.6566 & cubic inch per square foot $\left(\mathrm{in}^{3} / \mathrm{ft}^{2}\right)$ \\
meter per second $(\mathrm{m} / \mathrm{s})$ & 3.281 & foot per second $(\mathrm{ft} / \mathrm{s})$ \\
milligram $(\mathrm{mg})$ & 0.00003527 & ounce, avoirdupois $(\mathrm{oz})$ \\
milligram per square meter $\left(\mathrm{mg} / \mathrm{m}^{2}\right)$ & 0.000003278 & ounce per square foot $\left(\mathrm{oz} / \mathrm{ft}^{2}\right)$ \\
gram per square meter $\left(\mathrm{g} / \mathrm{m}^{2}\right)$ & 0.003278 & ounce per square foot $\left(\mathrm{oz} / \mathrm{ft}^{2}\right)$ \\
\hline
\end{tabular}

Temperature in degrees Celsius $\left({ }^{\circ} \mathrm{C}\right)$ may be converted to degrees Fahrenheit $\left({ }^{\circ} \mathrm{F}\right)$ as follows:

$$
{ }^{\circ} \mathrm{F}=\left(1.8 x^{\circ} \mathrm{C}\right)+32 \text {. }
$$

Vertical coordinate information is referenced to the National Geodetic Vertical Datum of 1929 (NGVD 29).

Elevation, as used in this report, refers to distance above the vertical datum.

Horizontal coordinate information is referenced to the North American Datum of 1927 (NAD 27).

Specific conductance is given in microsiemens per centimeter at 25 degrees Celsius $(\mu \mathrm{S} / \mathrm{cm}$ at $\left.25^{\circ} \mathrm{C}\right)$.

Concentrations of chemical constituents in water are given in either milligrams per liter (mg/L) or micrograms per liter $(\mu \mathrm{g} / \mathrm{L})$. Concentrations of algal constituents are given in milligrams per square meter $\left(\mathrm{mg} / \mathrm{m}^{2}\right)$, grams per square meters $\left(\mathrm{g} / \mathrm{m}^{2}\right)$, billion cells per square meter, or cubic centimeters per square meter $\left(\mathrm{cm}^{3} / \mathrm{m}^{2}\right)$.

The water year begins 0ctober 1 and ends September 30, and is designated by the year in which it ends. For example, water year 2005 begins October 1, 2004, and ends September 30, 2005.

\section{Abbreviations, Initializms, and Acronyms}

$\begin{array}{ll}< & \text { less than } \\ \leq & \text { less than or equal to } \\ > & \text { greater than } \\ \geq & \text { greater than or equal to } \\ \text { AFDM } & \text { ash-free dry mass } \\ \text { ANOVA } & \text { analysis of variance } \\ \text { ATG } & \text { Aquatic Task Group } \\ \text { BLM } & \text { Bureau of Land Management } \\ \text { CBNG } & \text { coalbed natural gas } \\ \text { D50 } & \text { diameter of the 50th percentile (median) of particles } \\ \text { D84 } & \text { diameter of the 84th percentile of particles } \\ \text { IBI } & \text { Index of Biotic Integrity }\end{array}$




$\begin{array}{ll}\text { IWG } & \text { Interagency Working Group } \\ \text { MDEQ } & \text { Montana Department of Environmental Quality } \\ \text { NMDS } & \text { nonmetric multidimensional scaling } \\ \mathrm{p} & \text { probability level } \\ \text { PC } & \text { principal components } \\ \text { PCA } & \text { principal components analysis } \\ \text { PRMS } & \text { Powder River main stem } \\ \text { OC } & \text { quality control } \\ r & \text { correlation coefficient } \\ \text { TRMT } & \text { Tongue River main stem and mountainous tributaries } \\ \text { TRPT } & \text { Tongue River plains tributaries } \\ \text { USEPA } & \text { U.S. Environmental Protection Agency } \\ \text { USGS } & \text { U.S. Geological Survey } \\ \text { WDEO } & \text { Wyoming Department of Environmental Quality } \\ \text { WGFD } & \text { Wyoming Game and Fish Department }\end{array}$





\title{
Assessment of Ecological Conditions and Potential Effects of Water Produced from Coalbed Natural Gas Development on Biological Communities in Streams of the Powder River Structural Basin, Wyoming and Montana, 2005-08
}

\author{
By David A. Peterson, Melanie L. Clark, Katharine Foster, Peter R. Wright, and Gregory K. Boughton
}

\section{Abstract}

Ongoing development of coalbed natural gas in the Powder River structural basin in Wyoming and Montana led to formation of an interagency task group to address concerns about the effects of the resulting production water on biological communities in streams of the area. The interagency task group developed a monitoring plan and conducted sampling of macroinvertebrate, algal, and fish communities at 47 sites during 2005-08 to document current ecological conditions and determine existing and potential effects of water produced from coalbed natural gas development on biological communities.

Macroinvertebrate, algal, and fish community composition varied between drainage basins, among sites within drainage basins, and by year. Macroinvertebrate communities of the main-stem Tongue River were characterized by higher taxa richness and higher abundance of Ephemeroptera, for example, compared to macroinvertebrate communities in plains tributaries of the Tongue River and the main-stem Powder River. Fish communities of the Tongue River were characterized by higher taxa richness and abundance of introduced species compared to the Powder River where native species were dominant.

Macroinvertebrate community metric values from sites in the middle reach of the main-stem Powder River, from below Willow Creek to below Crazy Woman Creek, differed from metric values in the upper and lower reaches of the Powder River. Metrics indicative of communitywide differences included measures of taxa richness, relative abundance, feeding mode, and tolerance. Some of the variation in the macroinvertebrate communities could be explained by variation in environmental variables, including physical (turbidity, embeddedness, bed substrate size, and streamflow) and chemical (alkalinity and specific conductance) variables. Of these environmental variables, alkalinity was the best indicator of coalbed natural gas development because of the sodiumbicarbonate signature of the production water.
Algal samples from the main-stem Powder River generally confirmed the pattern observed in the macroinvertebrate communities. Algal communities at sites in the middle reach of the Powder River commonly were characterized by dominance by a single taxon and by low biovolume of algae compared to other sites.

In contrast to the macroinvertebrate and algal communities, species richness of fish communities was highest in the middle reach of the Powder River. Although a few significant differences in fish metrics were determined along the main-stem Powder River, the differences did not correspond to the pattern observed for the macroinvertebrate and algae communities.

Differences in biological communities were noted between years, potentially due to the effects of drought. Macroinvertebrate community metrics, such as Diptera taxa richness, were significantly different in the severe drought year of 2006 from metric values in 2005 and 2007-08. Waterquality data collected during the study indicated that, with few exceptions, water-quality constituents generally did not exceed State or Federal acute and chronic criteria for the protection of aquatic life.

\section{Introduction}

Development of energy and mineral resources in the Powder River structural basin in northeastern Wyoming and southeastern Montana (fig. 1) includes coalbed natural gas, conventional oil and gas, and coal mining. A common theme of coalbed natural gas (CBNG) development is discharge of groundwater that commonly is saline or unsuitable for irrigation of crops and has unknown effects on the aquatic communities inhabiting streams that receive the water (Bureau of Land Management, 2009). 


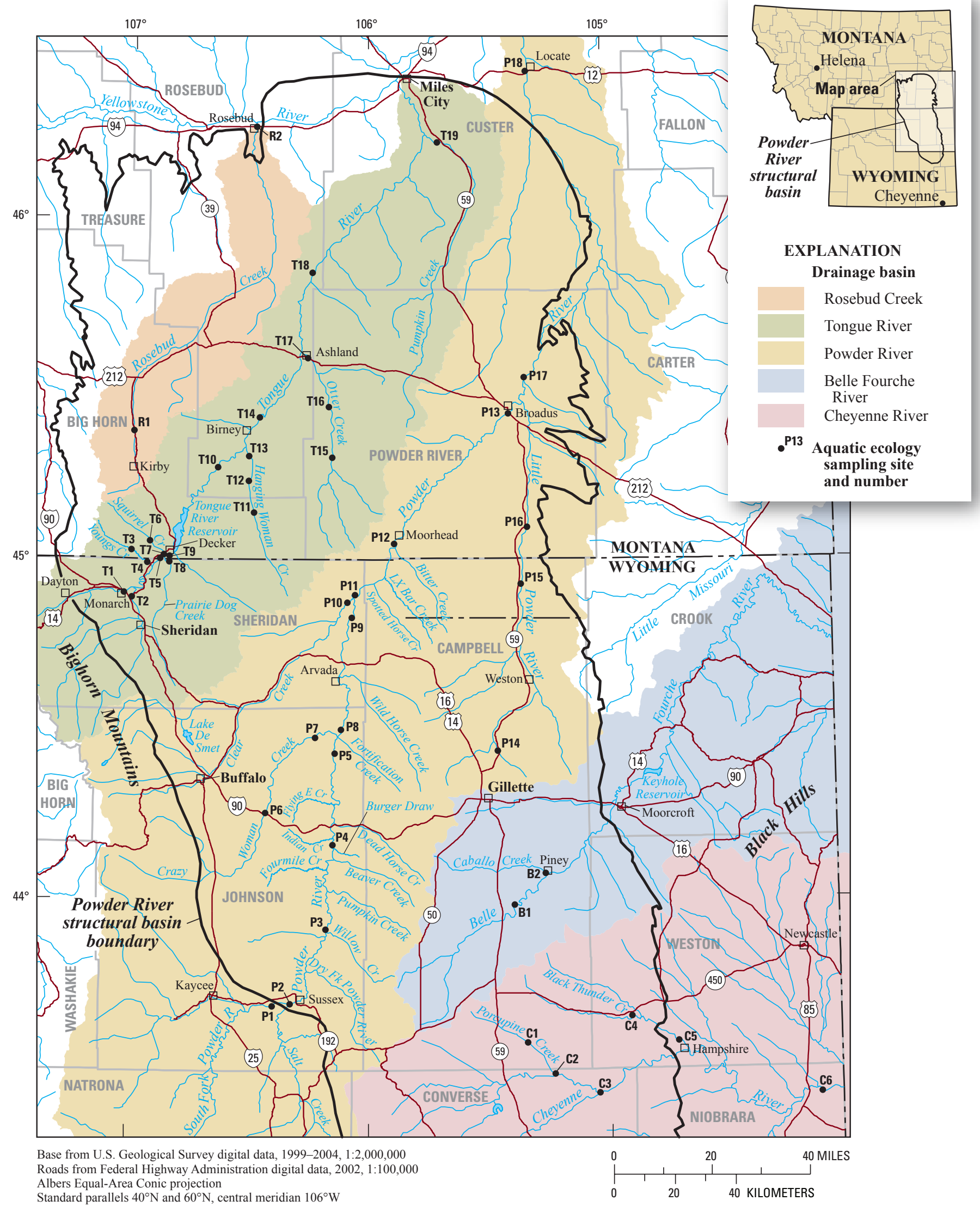

Figure 1. Location of aquatic ecology sampling sites in the Powder River structural basin, Wyoming and Montana, 2005-08. 
A total of 44,564 CBNG wells have been permitted in the Wyoming part of the study area as of September 30, 2008, and about one-half $(19,172)$ of those wells were producing water (fig. 2; Wyoming Oil and Gas Conservation Commission, 2010). Comparison of the cumulative production data from 2006 to those from 2008 (fig. 2) indicated that most of the development during 2007-08 occurred in the Powder River and Tongue River drainage basins. For example, cumulative water production in the Powder River drainage in Wyoming during 2008 was more than twice the amount of cumulative water production in 2006.

Substantial CBNG resources also exist in the Montana part of the study area but are largely undeveloped compared to Wyoming. Permit data from the Montana Department of Environmental Quality (MDEQ) (written commun., 2010) indicate cumulative CBNG water production as of October 2008 was about 19,237 acre-feet (acre-ft) from 150 or more wells in the Tongue River drainage basin in Montana. At maximum development in the study area, CBNG wells are expected to number 60,000 in Wyoming and more than 10,000 in Montana (Stricker and others, 2006).

To address concerns about the potential effects of CBNG development on cultural and natural resources, the U.S. Department of the Interior Bureau of Land Management (BLM) formed an Interagency Working Group (IWG) of Federal, State, and tribal agencies. The charter of the IWG states that it "... was established as the forum for government agencies to address, discuss, and find solutions to issues of common concern to all parties involved in permitting and monitoring of CBNG development" (Powder River Natural Gas Interagency Working Group, 2004). The IWG charter also provides for establishment of working groups to address technical issues as envisioned by the April 2003 Record of Decision (Bureau of Land Management, 2003). One working group, the Aquatic Task Group (ATG), was tasked with assessing potential effects of $\mathrm{CBNG}$ produced water on aquatic ecological resources. Agencies involved in the ATG include the BLM, the Wyoming Department of Environmental Quality (WDEQ), MDEQ, the Wyoming Game and Fish Department, the Montana Department of Fish, Wildlife, and Parks, and the U.S. Environmental Protection Agency (USEPA).

The ATG developed a monitoring plan to meet two main objectives: (1) establish current ecological conditions for aquatic biota and their habitat and (2) determine existing and potential effects of CBNG produced water on aquatic life (Bureau of Land Management, 2009). The assessment of current ecological conditions as of 2005-08 was performed by the U.S. Geological Survey (USGS) under the direction of the ATG. The determination of effects from CBNG-produced water is addressed in part by the current conditions data assessment, as well as by studies of potential effects of CBNG water on fish communities in the study area (Davis, 2008; Skaar and others, 2006) and a literature review of the effects of CBNG activities on fish communities (Davis and others, 2009). Additional information about CBNG development and monitoring is available from the BLM at http://www.wy.blm.gov/prbgroup/; the WDEQ at http://deq.state.wy.us/wqd/WYPDES_Permitting/WYPDES_cbm/cbm.asp; and the USGS at $h t t p: / / w y . w a t e r . u s g s . g o v /$.

\section{Purpose and Scope}

This report is a companion report to Peterson and others (2009) that was prepared in cooperation with the ATG and that described conditions for biological communities (macroinvertebrates, algae, and fish) for 2005-06 at 47 sites (fig. 1; table 1). Data collection continued in 2007-08 at these same 47 sites and included environmental variables (water quality and habitat) and biological communities (macroinvertebrate and algae sampling in 2007 and macroinvertebrate and fish sampling in 2008), but the 2007-08 data had not been published previous to this report. The types of samples and number of sites sampled varied annually due to drought and program constraints.

The purpose of this report is to (1) assess the current (2005-08) ecological conditions for environmental variables and biological communities, using new data from 2007-08 in conjunction with previously published data from 2005-06, and (2) describe potential effects of natural and anthropogenic environmental variables, including CBNG-produced water, on biological communities to the extent possible from the current conditions data.

For the sake of brevity, some of the previously published material (Peterson and others, 2009) is either omitted from this report or summarized and referenced. For example, the methods of sample collection and analysis in 2007-08 were the same as those used in 2005-06; therefore, descriptions of some collection or analytical methods that were previously published in Peterson and others (2009) are omitted from this report. Additional information about the description of the study area, reachwide habitat data from 2005, macroinvertebrate models from 2005-06, and seasonal fish community data from 2004-06 may be found in Peterson and others (2009).

\section{Methods of Data Analysis}

Biological community metrics were calculated using procedures and attributes described by Cuffney (2003) for macroinvertebrates, Porter (2008) for algae, and Bramblett and others (2005) for fish. Macroinvertebrate tolerance scores (Hilsenhoff, 1987; Cuffney, 2003) were assigned to one of three ranges: intolerant, greater than or equal to $(\geq) 0$ to less than or equal to $(\leq)$ 4; moderately tolerant, greater than $(>) 4$ to less than $(<) 7$; and tolerant, $\geq 7$ to $\leq 10$. Statistical comparisons of macroinvertebrate and fish community metrics were performed in S+ (TIBCO Software Inc., 2008) at a probability level (p) of 0.05 . Following procedures described by Helsel and Hirsch (2002), KruskalWallis rank sum tests were used to test for significant differences in metrics between sites and between years, and metrics identified as containing significant differences were then subjected to further evaluation using analysis of variance (ANOVA) and Tukey's test for multiple comparisons on rank-transformed data. 


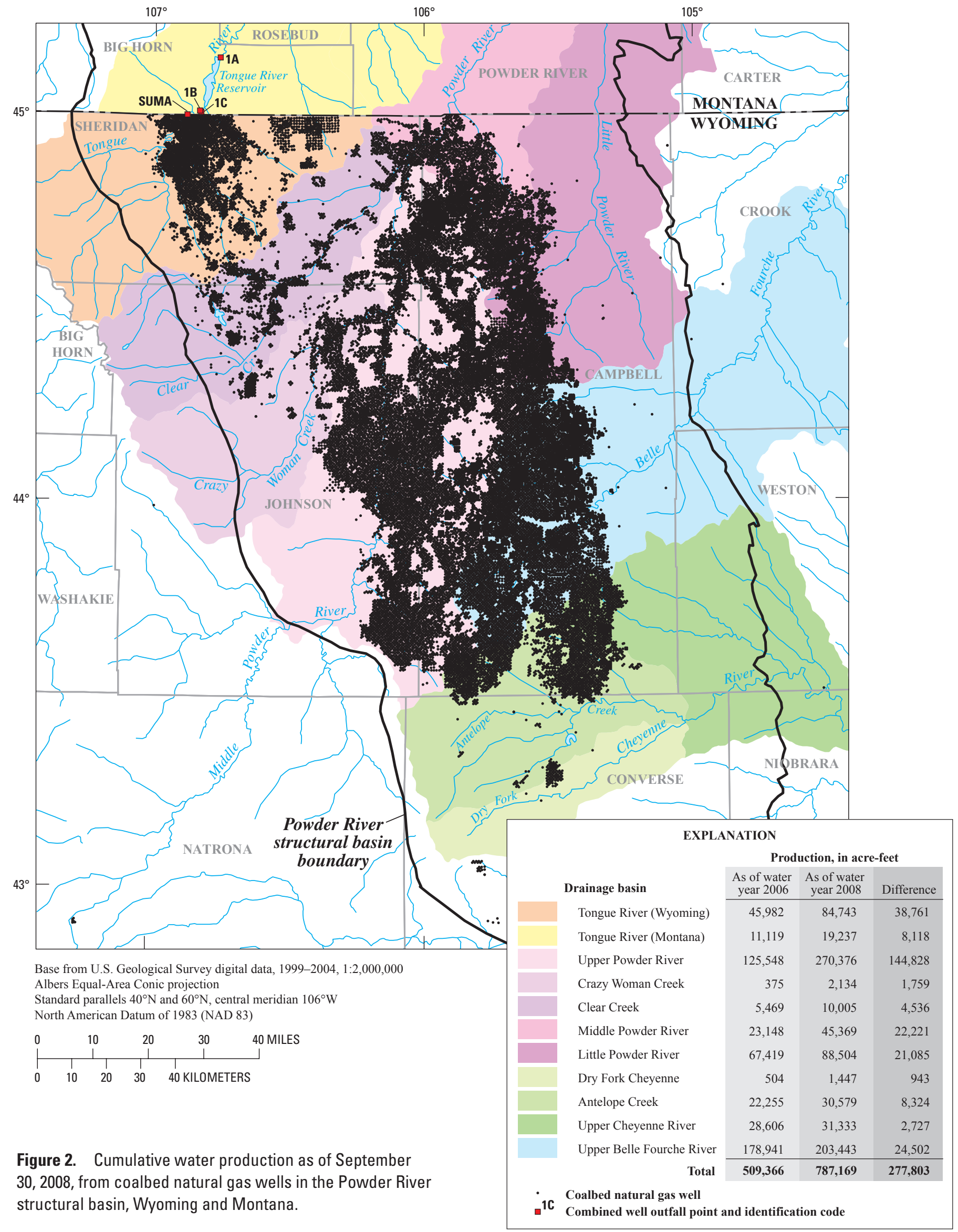


Table 1. Ecological sampling sites on streams in the Powder River structural basin, Wyoming and Montana, 2005-08.

[Shaded cells indicate main-stem sampling sites on the Tongue or Powder River. DMS, degrees, minutes, seconds; km², square kilometers; m, meters; P, plains; M, mountains]

\begin{tabular}{|c|c|c|c|c|c|c|c|c|c|}
\hline $\begin{array}{l}\text { Site } \\
\text { number } \\
\text { (fig. 1) }\end{array}$ & $\begin{array}{c}\text { U.S. Geological } \\
\text { Survey site } \\
\text { identification number }\end{array}$ & Site name & $\begin{array}{l}\text { Abbreviated } \\
\text { site name }\end{array}$ & $\begin{array}{l}\text { Latitude } \\
\text { (DMS) }\end{array}$ & $\begin{array}{l}\text { Longitude } \\
\text { (DMS) }\end{array}$ & $\begin{array}{c}\text { Drainage } \\
\text { area } \\
\left(\mathrm{km}^{2}\right)\end{array}$ & $\begin{array}{l}\text { Elevation } \\
\text { (m) }\end{array}$ & $\begin{array}{l}\text { Stream } \\
\text { head- } \\
\text { waters }\end{array}$ & $\begin{array}{c}\text { Reach } \\
\text { length } \\
\text { (m) }\end{array}$ \\
\hline R1 & 06295113 & Rosebud Creek at reservation boundary, near Kirby, MT & Upper Rosebud Creek & 452140 & 1065923 & 319 & 1,152 & $\mathrm{P}$ & 200 \\
\hline R2 & 06296003 & Rosebud Creek at mouth, near Rosebud, MT & Rosebud Creek at mouth & 461553 & 1062830 & 3,372 & 756 & $\mathrm{P}$ & 200 \\
\hline T1 & 06299980 & Tongue River at Monarch, WY & Tongue River at Monarch & 445401 & 1070113 & 1,238 & 1,103 & M & 970 \\
\hline $\mathrm{T} 2$ & 06305700 & Goose Creek near Acme, WY & Goose Creek & 445311 & 1065918 & 1,070 & 1,103 & M & 640 \\
\hline T3 & 450137106595101 & Youngs Creek near reservation boundary, near Decker, MT & Upper Youngs Creek & 450137 & 1065915 & 56 & 1,155 & $\mathrm{P}$ & 200 \\
\hline $\mathrm{T} 4$ & 445832106551401 & Youngs Creek above mouth, near Decker, MT & Youngs Creek at mouth & 445832 & 1065514 & 161 & 1,088 & $\mathrm{P}$ & 200 \\
\hline T5 & 445957106524701 & Tongue River below Youngs Creek, near Decker, MT & Tongue River below Youngs Creek & 445957 & 1065247 & 3,711 & 1,058 & M & 1,000 \\
\hline T6 & 06306100 & Squirrel Creek near Decker, MT & Upper Squirrel Creek & 450302 & 1065524 & 87 & 1,122 & $\mathrm{P}$ & 200 \\
\hline $\mathrm{T} 7$ & 450047106514201 & Squirrel Creek above mouth at Decker, MT & Squirrel Creek at mouth & 450047 & 1065142 & 112 & 972 & $\mathrm{P}$ & 200 \\
\hline $\mathrm{T} 8$ & 06306250 & Prairie Dog Creek near Acme, WY & Prairie Dog Creek & 445902 & 1065021 & 927 & 1,052 & $\mathrm{P}$ & 170 \\
\hline T9 & 06306300 & Tongue River at State line, near Decker, MT & Tongue River at State line & 450032 & 1065008 & 3,763 & 1,045 & M & 1,000 \\
\hline T10 & 451607106372801 & Tongue River at Prairie Dog Creek, near Decker, MT & Tongue River above Hanging Woman Creek & 451607 & 1063728 & 5,208 & 890 & M & 1,000 \\
\hline T11 & 06307570 & Hanging Woman Creek below Horse Creek, near Birney, MT & Upper Hanging Woman Creek & 450804 & 1062904 & 831 & 954 & $\mathrm{P}$ & 200 \\
\hline T12 & 451340106295501 & Hanging Woman Creek below Hay Gulch, near Birney, MT & Middle Hanging Woman Creek & 451344 & 1062957 & 958 & 997 & $\mathrm{P}$ & 200 \\
\hline T13 & 06307600 & Hanging Woman Creek near Birney, MT & Hanging Woman Creek at mouth & 451757 & 1063028 & 1,217 & 960 & $\mathrm{P}$ & 200 \\
\hline T14 & 06307616 & Tongue River at Birney Day School Bridge, near Birney, MT & Tongue River at Birney Day School & 452442 & 1062726 & 6,788 & 933 & M & 1,000 \\
\hline T15 & 451732106085001 & Otter Creek below Taylor Creek, near Otter, MT & Upper Otter Creek & 451732 & 1060853 & 803 & 991 & $\mathrm{P}$ & 200 \\
\hline T16 & 452642106091201 & Otter Creek below Tenmile Creek, near Ashland, MT & Middle Otter Creek & 452642 & 1060912 & 1,331 & 945 & $\mathrm{P}$ & 200 \\
\hline T17 & 06307740 & Otter Creek at Ashland, MT & Otter Creek at mouth & 453518 & 1061517 & 1,831 & 889 & $\mathrm{P}$ & 200 \\
\hline T18 & 06307830 & Tongue River below Brandenberg Bridge, near Ashland, MT & Tongue River below Brandenbe & 455023 & 1061309 & 10,225 & 841 & M & 1,000 \\
\hline T19 & 06308400 & Pumpkin Creek near Miles City, MT & Pumpkin Creek & 461342 & 1054124 & 1,805 & 759 & $\mathrm{P}$ & 200 \\
\hline P1 & 434056106244101 & Powder River above Lone Tree Draw, near Sussex, WY & Powder River above Salt Creek & 434056 & 1062441 & 5,828 & 1,350 & $\mathrm{P}$ & ${ }^{1} 3,218$ \\
\hline P2 & 434124106192401 & Powder River below Salt Creek, near Sussex, WY & Powder River below Salt Creek & 434124 & 1061924 & 7,980 & 1,338 & $\mathrm{P}$ & 13,218 \\
\hline P3 & 435453106104701 & Powder River below Willow Creek, near Sussex, WY & Powder River below Willow Creek & 435453 & 1061047 & 9,808 & 1,311 & $\mathrm{P}$ & 13,218 \\
\hline P4 & 440919106091401 & Powder River above Van Houghton Draw, near Buffalo, WY & Powder River below Burger Draw & 440919 & 1060914 & 11,111 & 1,216 & $\mathrm{P}$ & 13,218 \\
\hline P5 & 442538106082001 & Powder River below Mitchell Draw, near Arvada, WY & Powder River above Crazy Woman Creek & 442538 & 1060820 & 12,564 & 1,152 & $\mathrm{P}$ & 13,218 \\
\hline P6 & 441532106251301 & Crazy Woman Creek below I-90, near Buffalo, WY & Crazy Woman Creek below I-90 & 441532 & 1062513 & 1,769 & 1,280 & M & 340 \\
\hline P7 & 442817106133001 & Crazy Woman Creek near Upper Station, near Arvada, WY & Crazy Woman Creek near mouth & 442817 & 1061330 & 2,385 & 1,167 & M & 490 \\
\hline P8 & 443025106061601 & Powder River below Crazy Woman Creek, near Arvada, WY & Powder River below Crazy Woman Creek & 443025 & 1060616 & 15,286 & 1,134 & $\mathrm{P}$ & ${ }^{13}, 218$ \\
\hline P9 & 444857106030401 & Powder River above Ivy Creek, near Arvada, WY & Powder River above Clear Creek & 444857 & 1060304 & 17,050 & 1,062 & $\mathrm{P}$ & ${ }^{13}, 218$ \\
\hline P10 & 06324000 & Clear Creek near Arvada, WY & Clear Creek & 445218 & 1060456 & 2,875 & 1,069 & M & 750 \\
\hline P11 & 445339106032501 & Powder River below Clear Creek, near Arvada, WY & Powder River below Clear Creek & 445339 & 1060325 & 20,106 & 1,059 & $\mathrm{P}$ & 13,218 \\
\hline P12 & 06324500 & Powder River at Moorhead, MT & Powder River at Moorhead & 450328 & 1055239 & 20,943 & 1,021 & $\mathrm{P}$ & ${ }^{1} 3,218$ \\
\hline P13 & 06324710 & Powder River at Broadus, MT & Powder River at Broadus & 452537 & 1052405 & 22,657 & 919 & $\mathrm{P}$ & 13,218 \\
\hline P14 & 06324790 & Little Powder River at State Hwy 59, near Gillette, WY & Little Powder River at Highway 59 & 442609 & 1052717 & 110 & 1,250 & $\mathrm{P}$ & 200 \\
\hline P15 & 06324970 & Little Powder River above Dry Creek, near Weston, WY & Little Powder River above Dry Creek & 445537 & 1052110 & 3,204 & 1,039 & $\mathrm{P}$ & 240 \\
\hline P16 & 06325000 & Little Powder River at Biddle, MT & Little Powder River at Biddle & 450617 & 1051951 & 3,991 & 991 & $\mathrm{P}$ & 200 \\
\hline P17 & 453209105201201 & Powder River below Little Powder River, near Broadus, MT & Powder River below Little Powder River & 453209 & 1052012 & 29,503 & 908 & $\mathrm{P}$ & 13,218 \\
\hline P18 & 06326500 & Powder River near Locate, MT & Powder River near Locate & 462548 & 1051834 & 33,846 & 727 & $\mathrm{P}$ & 13,218 \\
\hline $\mathrm{C} 1$ & 06364300 & Porcupine Creek near Teckla, WY & Porcupine Creek & 433441 & 1052019 & 204 & 1,428 & $\mathrm{P}$ & 200 \\
\hline $\mathrm{C} 2$ & 06364700 & Antelope Creek near Teckla, WY & Antelope Creek & 432908 & 1051339 & 2,484 & 1,366 & $\mathrm{P}$ & 280 \\
\hline $\mathrm{C} 3$ & 06365900 & Cheyenne River near Dull Center, WY & Cheyenne River near Dull Center & 432545 & 1050243 & 3,955 & 1,314 & $\mathrm{P}$ & 320 \\
\hline $\mathrm{C} 4$ & 06375600 & Little Thunder Creek near Hampshire, WY & Little Thunder Creek & 433920 & 1045420 & 606 & 1,341 & $\mathrm{P}$ & 200 \\
\hline $\mathrm{C} 5$ & 06376300 & Black Thunder Creek near Hampshire, WY & Black Thunder Creek & 433454 & 1044311 & 1,386 & 1,244 & $\mathrm{P}$ & 200 \\
\hline C6 & 06386500 & Cheyenne River near Spencer, WY & Cheyenne River near Spencer & 432520 & 1040736 & 13,649 & 1,105 & $\mathrm{P}$ & 220 \\
\hline B1 & 06425720 & Belle Fourche River below Rattlesnake Creek, near Piney, WY & Belle Fourche River & 435904 & 1052316 & 1,282 & 1,384 & $\mathrm{P}$ & 200 \\
\hline B2 & 06425900 & Caballo Creek at mouth, near Piney, WY & Caballo Creek & 440448 & 1051559 & 673 & 1,335 & $\mathrm{P}$ & 200 \\
\hline
\end{tabular}

${ }^{1}$ Established reach length of 3,218 meters is maximum reach length; the actual reach length varied with each site visit depending on location of random starting point and availability of expected habitat types. 
Nonmetric multidimensional scaling (NMDS) ordinations of macroinvertebrate and algal community taxonomic data were prepared in PRIMER (Clarke and Gorley, 2006). Macroinvertebrate and algal abundance data were log transformed $(\log x+1)$ to approximate normality. Bray-Curtis similarity coefficients (Bray and Curtis, 1957) were computed to determine taxonomic (dis-)similarity among samples. NMDS ordinations of the Bray-Curtis similarity coefficients were used to determine relations among sites on the basis of the taxonomic composition of the communities.

Macroinvertebrate community data from samples collected in riffles were tested for relations with environmental variables using principal components analysis (PCA) and the BEST routine (Clarke and Gorley, 2006). Environmental data were $\log$ transformed $(\log x+1)$ and standardized prior to running PCA to identify the most important variables distinguishing the sites (highest eigenvectors) and to identify and remove collinear (highly correlated) variables. The environmental variables selected through PCA were tested for correlation with the taxonomic composition of the macroinvertebrate communities using the BEST routine in PRIMER (Clarke and Gorley, 2006). The BEST routine tests multiple combinations of the environmental variables against the Bray-Curtis similarity coefficients of the macroinvertebrate communities to determine the highest Spearman correlation coefficients (r) using five or fewer environmental variables.

Data for major ions were either collected at the time of biological sampling or, in the case of sites that are sampled within the water-quality monitoring network, were the most recently collected samples (usually within 30 days prior) at each site. Missing values for turbidity or dissolved oxygen (less than 5 percent of total observations) were estimated for the statistical analyses from average values at the site or nearby sites on the same stream.

\section{Quality Assurance}

Quality assurance for analyses of major ions, chlorophyll, and ash-free dry mass (AFDM) included collection and analysis of quality-control (QC) samples from selected sites. The relative percent difference (RPD) in constituent concentrations between the environmental (sample 1) and the QC (sample 2) samples was calculated using the formula:

$$
\begin{gathered}
\mathrm{RPD}=\text { absolute value }((\text { sample } 1-\text { sample } 2) / \\
[(\text { sample } 1+\text { sample } 2) / 2]) \times 100
\end{gathered}
$$

The RPD for major ions in six pairs of environmental and QC samples was less than 5 percent, on the average. The RPD for chlorophyll and AFDM in four pairs of samples was less than 15 percent, on the average.
Quality assurance for the biological taxonomic analyses also included collection and analysis of QC samples for macroinvertebrates and algae. The taxonomic data do not lend themselves to conventional QC analyses such as RPD; therefore, the taxonomic QC samples were compared to the parent samples using Bray-Curtis similarity coefficients. The similarity coefficients of the macroinvertebrate and algae QC samples ranged from 46 to 83 percent (table 2), on a scale from 0 (no similarity) to 100 percent (complete similarity). The similarity coefficients between the parent and QC samples were higher than similarities with other environmental samples as shown in ordinations of the 2007-08 data in the following sections "Macroinvertebrate Community Composition" and "Composition" under the "Algal Communities" section and ordinations of the 2005-06 data (Peterson and others, 2009, their figs. 20 and 31).

Quality assurance for fish taxonomy was provided through voucher specimens and photographic vouchers collected as per the fish taxonomy quality-assurance plan (Walsh and Meador, 1998). Voucher specimens were sent to Dr. Robert Bramblett at Montana State University, Bozeman, Montana, for taxonomic confirmation and then sent to the Museum of Southwestern Biology at the University of New Mexico, Albuquerque, New Mexico, for curation.

\section{Assessment of Ecological Conditions}

This section of the report describes the assessment of current (2005-08) ecological conditions, including water quality, habitat, macroinvertebrate communities, algal communities, and fish communities, for streams in the Powder River structural basin. A description of the hydrology of the Powder River structural basin also is included.

\section{Hydrology}

Streamflow data were collected for the sites listed in table 1 during 2005-08 and are available from http://waterdata.usgs.gov/wy/nwis/sw. Streams with mountainous headwaters (table 1), such as the Tongue River, Goose Creek, and Clear Creek, tended to have perennial flow during 2005-08, whereas streams with plains headwaters, such as those in the Cheyenne and Belle Fourche River drainage basins and the upper parts of Otter Creek and Hanging Woman Creek (Tongue River drainage basin), tended to have ephemeral or intermittent streamflow. Mean values of instantaneous streamflow at the time of ecological sampling during 2005-08 from sites on the main-stem Tongue River indicated streamflow was less upstream from Tongue River Reservoir (sites T1, T5, and T9; fig. $3 A$ ) than downstream (sites T10, T14, and T18). Mean values of instantaneous streamflow 
Table 2. Biological quality-control samples, Powder River structural basin, Wyoming and Montana, 2005-08.

\begin{tabular}{cllcc}
\hline $\begin{array}{c}\text { Site } \\
\text { number } \\
\text { (fig. 1) }\end{array}$ & \multicolumn{1}{c}{$\begin{array}{c}\text { Abbreviated } \\
\text { site name }\end{array}$} & $\begin{array}{c}\text { Sample } \\
\text { date }\end{array}$ & \multicolumn{1}{c}{$\begin{array}{c}\text { Sample } \\
\text { type }\end{array}$} & $\begin{array}{c}\text { Bray-Curtis } \\
\text { similarity } \\
\text { (percent) }\end{array}$ \\
\hline T1 & Tongue River at Monarch & $8 / 15 / 2005$ & Macroinvertebrate & Replicate \\
T1 & Tongue River at Monarch & $8 / 15 / 2005$ & Algae & Replicate \\
T3 & Upper Youngs Creek & $6 / 26 / 2008$ & Macroinvertebrate & Split \\
T7 & Squirrel Creek at mouth & $7 / 12 / 2007$ & Macroinvertebrate & Replicate \\
T17 & Otter Creek at mouth & $6 / 19 / 2007$ & Algae & Replicate \\
P6 & Crazy Woman Creek below I-90 & $7 / 11 / 2005$ & Macroinvertebrate & Replicate \\
P7 & Crazy Woman Creek near mouth & $7 / 12 / 2005$ & Algae & Replicate \\
P9 & Powder River above Clear Creek & $7 / 29 / 2008$ & Macroinvertebrate & Replicate \\
C4 & Little Thunder Creek & $6 / 13 / 2007$ & Algae & 61 \\
B2 & Caballo Creek & $6 / 28 / 2005$ & Algae & 62 \\
\end{tabular}

from sites on the main-stem Powder River indicated more flow within the vicinity of Clear Creek near Arvada, Wyo. (sites P9 and P11) to Moorhead, Mont. (site P12) than at reaches farther upstream or downstream (fig. $3 B$ ).

Throughout most of the study area, daily streamflow in the study area during 2007-08 tended to be near or above the long-term average values, in contrast to below-average values in 2005-06 (http://waterdata.usgs.gov/wy/nwis/sw/). Drought conditions prevailed in the study area during 2005-06 and for several years preceding the study (National Oceanic and Atmospheric Administration Satellite and Information Service, 2005). Instantaneous values of streamflow at the time of ecological sampling (table 3 ) indicated substantially less streamflow in 2006 than in 2005 or 2007-08 (figs. 3C and 3D). During the summer of 2006, some of the sampling sites on the main-stem Powder River (such as sites P8, P9, P12, and P17) did not have flow, with only isolated pools present to sample. Historically, infrequent days of no flow have been documented on the Powder River from Arvada, Wyo., to Moorhead, Mont. (Ringen and Daddow, 1990), which includes sites P9, P11, and P12 (fig. 1) and which may be inherent to the reach under natural conditions.

\section{Water Quality}

Water-quality data (http://waterdata.usgs.gov/wy/nwis/qw) that were collected during water years $2005-08$ as part of ATG sampling events, as well as data for samples collected at ATG study sites that are part of other USGS monitoring efforts, were compiled for 47 study sites in the five major drainage basins of Rosebud Creek, Tongue River, Powder River, Cheyenne River, and Belle Fourche River. The water year 2005-08 data were compared to applicable State or Federal aquatic-life criteria for selected water-quality constituents and are statistically summarized in the Appendix. A subset, consisting of water-quality data collected during ecological sampling events on the Tongue River and Powder River, was used to describe how conditions varied on the two large main-stem rivers during relatively lowflow conditions of summer.

\section{Water-Quality Characteristics}

Water-quality characteristics of streams in the Powder River structural basin are variable largely because of streamflow and geology (Clark and Mason, 2007). The Tongue River drainage basin has its headwaters in the Bighorn Mountains, which are characterized by relatively high precipitation, low evaporation, and resistant igneous, metamorphic, and Paleozoic-era sedimentary rocks (Clark and Mason, 2007). In contrast, the Powder River drainage basin has a large part of its headwaters in the plains, which are characterized by relatively low precipitation, high evaporation, and soluble Tertiary-age sedimentary rocks (Clark and Mason, 2007). Different basin characteristics of the Tongue River and Powder River are reflected in the range of mean values for specific conductance, dissolved sodium, and alkalinity for ATG sampling events during the relatively low flow conditions of summer (fig. 4; table 3). Specific conductance, dissolved sodium, and alkalinity are general indicators of water-quality characteristics and also have been identified as constituents of concern for CBNG discharges because their concentrations in produced water can be large (Rice and others, 2000).

Mean specific conductance values at the time of ecological sampling for the Tongue River (456 to 756 microsiemens per centimeter at 25 degrees Celsius $[\mu \mathrm{S} / \mathrm{cm}])$ were small compared to the Powder River (1,970 to $4,750 \mu \mathrm{S} / \mathrm{cm})$ (fig. 4). Mean specific conductance values for the Tongue River increased from Monarch, Wyo. (site T1) to the State line near Decker, Mont. (site T9), with a decrease downstream from Tongue River Reservoir (site T10) followed by another increase downstream to below Otter Creek (site T18). Mean sodium and alkalinity values on the Tongue River generally had a similar spatial pattern as specific conductance, with the largest concentrations at site T9. Potential anthropogenic influences to water quality of the Tongue River between sites T1 and T9 include $\mathrm{CBNG}$ produced water, irrigation return flows and diversions, treated wastewater effluent, and municipal stormwater runoff. 

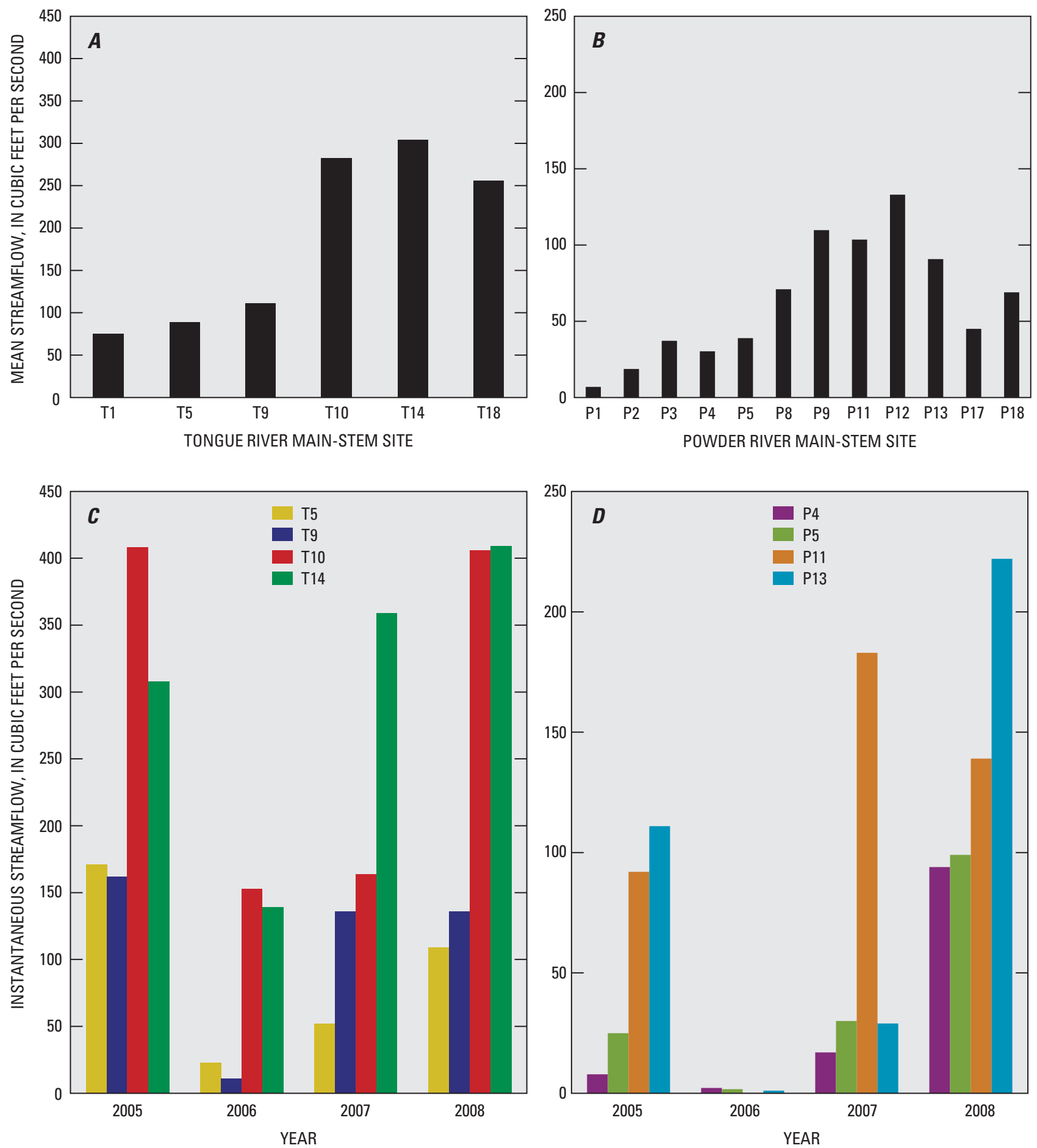

Figure 3. Streamflow at the time of ecological sampling-Mean streamflow values from 2005 to 2008 for sites on $(A)$ main-stem Tongue River, and $(B)$ main-stem Powder River; instantaneous streamflow values for selected sites on $(C)$ main-stem Tongue River, and $(D)$ main-stem Powder River, Wyoming and Montana. 
Mean specific conductance for the Powder River nearly doubled between sites P1 and P2 (fig. 4) near Sussex, Wyo., as a result of inputs from Salt Creek. No CBNG discharges occur upstream from sites P1 and P2 (Wyoming Department of Environmental Quality, written commun., 2010). The water quality in this reach of the Powder River is affected by produced water from conventional oil and gas production in the Salt Creek drainage basin (Clark and Mason, 2007). The influence of Salt Creek on the Powder River was particularly evident in mean dissolved sodium concentrations, which increased downstream from 284 to 797 milligrams per liter $(\mathrm{mg} / \mathrm{L})$ between sites P1 and P2. The influence of Salt Creek becomes less pronounced downstream, however, as indicated by a decrease in mean specific conductance and sodium concentrations downstream from Salt Creek (site P2) to site P11 downstream from Clear Creek. The decreases are likely due to the effects of dilution by increased streamflow. Among all sites, mean specific conductance and dissolved sodium concentrations were lower for the Powder River from Clear Creek near Arvada, Wyo., (site P9) to Moorhead, Mont. (site P12) than those for site P1, which is on the Powder River upstream from CBNG development. An increase in mean dissolved sodium concentrations was observed between Moorhead (site P12) and Broadus, Mont. (site P13). No CBNG discharges occur between sites P12 and P13 (Montana Department of Environmental Quality, written commun., 2010) and thus are not associated with the increase in specific conductance in this reach. The spatial pattern for alkalinity is different than the patterns for specific conductance and sodium. The largest mean value for alkalinity of $583 \mathrm{mg} / \mathrm{L}$ (as calcium carbonate) was for the Powder River below Burger Draw (site P4), which receives CBNG discharges from several drainages upstream. This value is about three times larger than the mean concentration calculated for the rest of the Powder River sites. Overall, mean values of alkalinity for the Powder River were less variable when compared to mean values of specific conductance and dissolved sodium concentrations, with the exception of site P4.

\section{Comparisons to Aquatic-Life Criteria}

The State of Wyoming (Wyoming Department of Environmental Quality, 2007), State of Montana (Montana Department of Environmental Quality, 2008), and USEPA (U.S. Environmental Protection Agency, 2005) have established chronic and acute criteria for the protection of aquatic life (table 4). Aquatic life includes fish, invertebrates, amphibians, and other organisms that inhabit streams at some stage in their life cycles. Acute criteria are based on a 1-hour average concentration, not to be exceeded more than once every 3 years. Chronic criteria are based on a 4-day average concentration, not to be exceeded more than once every 3 years. For this report, constituent concentrations were compared to Wyoming numeric criteria for sites that are physically located in Wyoming and to Montana criteria for sites that are physically located in Montana. The State of Wyoming aquatic-life criteria for trace elements generally are based on dissolved concentrations in contrast to the State of Montana aquaticlife criteria that generally are based on "total recoverable" (referred to as "total" in this report) concentrations. Total concentrations occur in water at higher concentrations than dissolved concentrations because total concentrations include particulate or colloidal phases of the constituent in addition to the dissolved phase. Montana ATG study sites may appear to have poorer water quality, therefore, compared to Wyoming ATG study sites because more Montana samples had concentrations that exceeded aquatic-life standards. Because dissolved and total concentrations can vary substantially for some trace elements, constituent concentrations for both States also were compared to the USEPA national recommended aquaticlife criteria for reference. Criteria for the protection of human health were not evaluated because the focus of this report is on aquatic life.

Some of the trace-element constituents that were analyzed in water-quality samples are classified as "priority pollutants," which are constituents that require water-quality criteria to be established by the USEPA, based on section 307 of the Federal Clean Water Act (table 4). For some constituents (arsenic, chromium [VI], mercury, and selenium), the toxicity threshold for acute and chronic values is the same for all waters. For other constituents (cadmium, chromium [III], lead, nickel, and zinc), the toxicity is hardness-dependent; therefore, sample-specific criteria were calculated for comparison. Values listed for these constituents (table 4) are for example only and are based on a hardness value of $100 \mathrm{mg} / \mathrm{L}$ (as calcium carbonate). The number of exceedances and sites described in this report for hardness dependent criteria were calculated from the hardness concentration associated with each individual sample. Unlike the State of Wyoming and State of Montana equations for copper, the USEPA national recommended aquatic-life criteria for dissolved copper are not a single hardness-dependent equation; instead, values are based on a complex biotic ligand model that includes $\mathrm{pH}$, hardness, and dissolved-organic carbon (U.S. Environmental Protection Agency, 2007). Chromium concentrations were compared to criteria for both chromium (III) and chromium (VI) for this report; however, the laboratory analyses for chromium were not speciated for valence states. Dissolvedmercury concentrations were not available for comparison with State of Wyoming or USEPA national recommended criteria. Total-mercury concentrations were available and were used for comparison in this report because if the total concentration is less than dissolved criteria, then the dissolved component must also have been less. The USEPA national recommended aquatic-life acute criteria for selenium currently are under review (U.S. Environmental Protection Agency, 2004). 
Table 3. Environmental variables associated with ecological samples, Powder River structural basin, Wyoming and Montana, $2005-08$.

[Shaded cells indicate main-stem sampling sites on the Tongue River or Powder River. m, meters; \%, percent; <, less than; mm, millimeters; $\mathrm{D}_{50}$, diameter of the 50th percentile of particles; $\mathrm{D}_{84}$, diameter of the 84 th percentile of particles; $\mathrm{ft}^{3} / \mathrm{s}$, cubic feet per second; $\mu \mathrm{S} / \mathrm{cm}$, microsiemens per centimeter at 25 degrees Celsius; ${ }^{\circ} \mathrm{C}$, degrees Celsius; $\mathrm{mg} / \mathrm{L}$, milligrams per liter; NTRU, nephelometric turbidity ratio units; NA, not applicable]

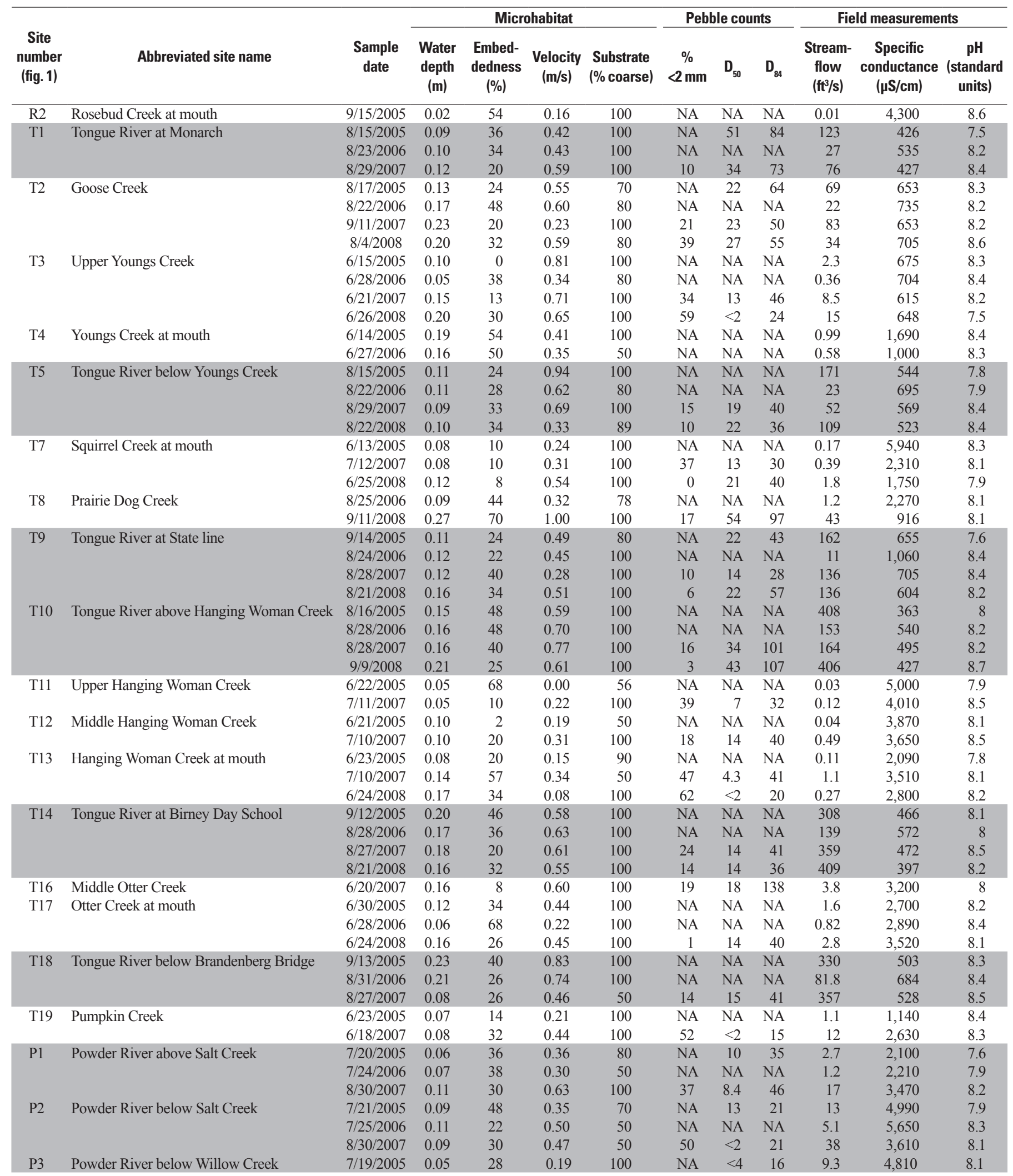


Table 3. Environmental variables associated with ecological samples, Powder River structural basin, Wyoming and Montana, 2005-08. - Continued

[Shaded cells indicate main-stem sampling sites on the Tongue River or Powder River. m, meters; \%, percent; <, less than; mm, millimeters; $\mathrm{D}_{50}$, diameter of the 50 th percentile of particles; $\mathrm{D}_{84}$, diameter of the 84 th percentile of particles; $\mathrm{ft} / \mathrm{s}$, cubic feet per second; $\mu \mathrm{S} / \mathrm{cm}$, microsiemens per centimeter at 25 degrees Celsius; ${ }^{\circ} \mathrm{C}$, degrees Celsius; $\mathrm{mg} / \mathrm{L}$, milligrams per liter; NTRU, nephelometric turbidity ratio units; NA, not applicable]

\begin{tabular}{|c|c|c|c|c|c|c|c|c|c|c|c|c|c|c|c|}
\hline \multirow[b]{2}{*}{$\begin{array}{c}\text { Site } \\
\text { number } \\
\text { (fig. 1) }\end{array}$} & \multicolumn{4}{|c|}{ Field measurements } & \multicolumn{11}{|c|}{ Dissolved major ion concentrations } \\
\hline & $\begin{array}{c}\text { Water } \\
\text { temper- } \\
\text { ature } \\
\left({ }^{\circ} \mathrm{C}\right)\end{array}$ & $\begin{array}{c}\text { Dissolved } \\
\text { oxygen } \\
\text { (mg/L) }\end{array}$ & $\begin{array}{c}\text { Turbidity } \\
\text { (NTRU) }\end{array}$ & $\begin{array}{c}\text { Site } \\
\text { number }\end{array}$ & $\begin{array}{c}\text { Calcium } \\
\text { (mg/L) }\end{array}$ & $\begin{array}{c}\text { Mag- } \\
\text { nesium } \\
(\mathrm{mg} / \mathrm{L})\end{array}$ & $\begin{array}{l}\text { Sodium } \\
\text { (mg/L) }\end{array}$ & $\begin{array}{l}\text { Sodium } \\
\text { adsorption } \\
\text { ratio }\end{array}$ & $\begin{array}{c}\text { Potas- } \\
\text { sium } \\
(\mathrm{mg} / \mathrm{L})\end{array}$ & $\begin{array}{c}\text { Alkalinity } \\
\text { (mg/L) }\end{array}$ & $\begin{array}{c}\text { Chloride } \\
\text { (mg/L) }\end{array}$ & $\begin{array}{l}\text { Sulfate } \\
\text { (mg/L) }\end{array}$ & $\begin{array}{c}\text { Fluoride } \\
\text { (mg/L) }\end{array}$ & $\begin{array}{l}\text { Silica } \\
\text { (mg/L) }\end{array}$ & $\begin{array}{c}\text { Total } \\
\text { dissolved } \\
\text { solids } \\
\text { (mg/L) }\end{array}$ \\
\hline R2 & 12.5 & 9.3 & 9.1 & R2 & 73.8 & 183 & 565 & 8 & 14.4 & 450 & 23.5 & 1,520 & 0.84 & 0.86 & 2,650 \\
\hline \multirow[t]{3}{*}{$\mathrm{T} 1$} & 14.8 & 7.5 & 30 & $\mathrm{~T} 1$ & 44.2 & 19.6 & 8.82 & 0.3 & 1.66 & 167 & 1.11 & 35.5 & 0.19 & 5.28 & 217 \\
\hline & 19.6 & 7.7 & 14 & $\mathrm{~T} 1$ & 44 & 28.9 & 19.1 & 0.5 & 2.55 & 209 & 1.96 & 67.2 & 0.23 & 3.83 & 293 \\
\hline & 19.8 & 11.6 & 11 & $\mathrm{~T} 1$ & 46.8 & 20.1 & 9.98 & 0.3 & 1.73 & 185 & 1.42 & 34.6 & 0.15 & 6.58 & 233 \\
\hline \multirow{2}{*}{$\mathrm{T} 2$} & 12 & 8.3 & 12 & $\mathrm{~T} 2$ & 62.8 & 44.5 & 30.8 & 0.7 & 3.86 & 260 & 9.95 & 141 & 0.32 & 3.87 & 454 \\
\hline & 25.2 & 10 & 3.8 & $\mathrm{~T} 2$ & 61.9 & 39.6 & 27.1 & 0.7 & 3.74 & 239 & 10 & 135 & 0.29 & 3.52 & 425 \\
\hline \multirow[t]{4}{*}{$\mathrm{T} 3$} & 19 & 7.6 & 62 & $\mathrm{~T} 3$ & 55.1 & 38.2 & 12.8 & 0.3 & 4.61 & 293 & 1.97 & 53.2 & 0.59 & 13.8 & 356 \\
\hline & 22 & 7.2 & 85 & $\mathrm{~T} 3$ & 62.5 & 51.1 & 17.2 & 0.4 & 7.17 & 373 & 1.58 & 30.4 & 0.66 & 18.8 & 413 \\
\hline & 18 & 8 & 42 & T3 & 65.6 & 36.8 & 10.5 & 0.3 & 3.96 & 312 & 1.66 & 38.2 & 0.49 & 17.7 & 362 \\
\hline & 19.5 & 7.3 & 59 & T3 & 67.8 & 41.8 & 12.4 & 0.3 & 5.55 & 336 & 1.58 & 38.6 & 0.51 & 16.9 & 387 \\
\hline T5 & 19 & 8.1 & 5 & T5 & 44.8 & 29.5 & 19.3 & 0.5 & 2.94 & 192 & 3.59 & 84.3 & 0.23 & 1.64 & 301 \\
\hline \multirow{3}{*}{$\mathrm{T} 7$} & 13 & 9.7 & 6.8 & $\mathrm{~T} 7$ & 153 & 463 & 835 & 7.6 & 20 & 590 & 12.7 & 3330 & 0.73 & 7.68 & 5,180 \\
\hline & 20 & NA & 1.2 & $\mathrm{~T} 7$ & 86.4 & 184 & 199 & 2.8 & 12.1 & 530 & 4.75 & 880 & 0.57 & 4.21 & 1,690 \\
\hline & 19.5 & 7.7 & 14 & $\mathrm{~T} 7$ & 92.2 & 146 & 113 & 1.7 & 9.23 & 485 & 3.64 & 587 & 0.52 & 9.29 & 1,250 \\
\hline \multirow[t]{2}{*}{$\mathrm{T} 8$} & 15.3 & 9.1 & 10 & $\mathrm{~T} 8$ & 157 & 136 & 202 & 2.9 & 11.4 & 370 & 7.77 & 1040 & 0.41 & 13.4 & 1,790 \\
\hline & 15.3 & 8.8 & 91 & $\mathrm{~T} 8$ & 72.4 & 39.4 & 33.5 & 0.8 & 4.24 & 198 & 2.48 & 220 & 0.21 & 10.2 & 501 \\
\hline \multirow[t]{4}{*}{ T9 } & 13.6 & 8.4 & 12 & T9 & 54.2 & 35.7 & 30.8 & 0.8 & 2.92 & 208 & 3.53 & 136 & 0.29 & 4.89 & 393 \\
\hline & 24.1 & 10.8 & 4.4 & T9 & 49.9 & 46 & 51.6 & 1.3 & 4.32 & 227 & 6.96 & 200 & 0.47 & 4.44 & 500 \\
\hline & 21 & 10.3 & 8.6 & T9 & 59.1 & 33 & 27.7 & 0.7 & 3.02 & 203 & 4.16 & 126 & 0.3 & 7.39 & 382 \\
\hline & 25 & 9.7 & 24 & T9 & 52.6 & 28.1 & 26.3 & 0.7 & 2.85 & 224 & 4.24 & 109 & 0.3 & 3.53 & 361 \\
\hline $\mathrm{T} 10$ & 20 & 8 & 13 & $\mathrm{~T} 10$ & 37.2 & 17 & 14.7 & 0.5 & 1.92 & 130 & 1.46 & 54.4 & 0.18 & 3.61 & 209 \\
\hline & 18.5 & 7.5 & 0.6 & $\mathrm{~T} 10$ & 36.2 & 28.8 & 36.5 & 1.1 & 3.62 & 170 & 3.4 & 111 & 0.3 & 0.67 & 322 \\
\hline & 19 & 6.7 & 3.4 & T10 & 48.6 & 24.2 & 23.9 & 0.7 & 2.91 & 174 & 2.7 & 87.6 & 0.24 & 3.14 & 298 \\
\hline T14 & 18.5 & 8.3 & 10 & T14 & 44.9 & 22.3 & 21.2 & 0.6 & 2.54 & 160 & 2.23 & 81.7 & 0.23 & 2.02 & 273 \\
\hline & 23.5 & 10.5 & NA & T14 & 33.7 & 29 & 35.6 & 1.1 & 3.5 & 175 & 3.39 & 116 & 0.3 & 1.81 & 328 \\
\hline & 22.5 & 8.5 & 7 & T14 & 33.6 & 16.9 & 14.9 & 0.5 & 2.41 & 124 & 1.75 & 56.6 & 0.19 & 6.27 & 207 \\
\hline & 24.5 & 8.2 & 14 & T14 & 34.7 & 15.4 & 14.5 & 0.5 & 2.16 & 128 & 1.61 & 51.6 & 0.17 & 5.57 & 203 \\
\hline T16 & 19.5 & 7.5 & 2.5 & T16 & 129 & 196 & 391 & 5.1 & 19.4 & 502 & 10.7 & 1,440 & 0.66 & 7.43 & 2,490 \\
\hline $\mathrm{T} 17$ & 18 & 5.3 & 97 & T17 & 87.3 & 180 & 442 & 6.2 & 20.1 & 592 & 12.3 & 1,260 & 0.95 & 14.2 & 2,370 \\
\hline & 22.2 & 5 & 79 & T17 & 55.8 & 141 & 394 & 6.4 & 19 & 579 & 11.4 & 1,020 & 1 & 10.6 & 2,000 \\
\hline & 21.5 & 5.9 & 21 & T17 & 84.8 & 147 & 406 & 6.2 & 19.7 & 490 & 12.1 & 1,290 & 0.78 & 6.44 & 2,260 \\
\hline T18 & 14.5 & 9.3 & 16 & T18 & 45 & 23.6 & 23.7 & 0.7 & 2.87 & 156 & 2.51 & 89.6 & 0.24 & 1.81 & 283 \\
\hline & 17.8 & 8.6 & NA & T18 & 38.9 & 32.4 & 44.5 & 1.3 & 3.75 & 190 & 4.05 & 142 & 0.33 & 4.61 & 385 \\
\hline & 20 & 8 & 11 & T18 & 42.1 & 21.8 & 25.7 & 0.8 & 2.94 & 165 & 2.53 & 95.6 & 0.24 & 5.7 & 296 \\
\hline T19 & 29.4 & NA & 158 & T19 & 32 & 14.5 & 181 & 6.7 & 8.69 & 214 & 3.49 & 294 & 0.41 & 9.93 & 673 \\
\hline & 21 & 8.7 & 69 & T19 & 64.2 & 65.8 & 334 & 7 & 10.4 & 281 & 6.58 & 853 & 0.34 & 8.1 & 1,510 \\
\hline P1 & 21.8 & 7.4 & 4.7 & P1 & 175 & 74.7 & 209 & 3.3 & 7.33 & 177 & 92.5 & 722 & 0.48 & 11.9 & 1,400 \\
\hline & 28.5 & 6.8 & 19 & P1 & 170 & 70.8 & 213 & 3.5 & 8.14 & 225 & 93.3 & 798 & 0.46 & 4.93 & 1,490 \\
\hline & 27 & 10.5 & 380 & P1 & 287 & 89.4 & 430 & 5.7 & 14.2 & 181 & 139 & 1,610 & 0.83 & 7.03 & 2,680 \\
\hline P2 & 23 & 7.7 & 16 & P2 & 113 & 75.4 & 932 & 17 & 20.1 & 288 & 943 & 1,070 & 1.39 & 7.97 & 3,340 \\
\hline & 32.7 & 7.3 & 12 & P2 & 140 & 79 & 906 & 15 & 28.7 & 175 & 915 & 1,310 & 1.99 & 4.46 & 3,490 \\
\hline & 22.1 & 10.2 & 370 & P2 & 177 & 69.7 & 554 & 8.9 & 15.6 & 238 & 444 & 1,030 & 1.03 & 12.5 & 2,450 \\
\hline P3 & 24.3 & 7.2 & 2.9 & P3 & 183 & 111 & 843 & 12 & 19.8 & 187 & 733 & 1,330 & 0.96 & 9.27 & 3,350 \\
\hline
\end{tabular}


Table 3. Environmental variables associated with ecological samples, Powder River structural basin, Wyoming and Montana, 2005-08. -Continued

[Shaded cells indicate main-stem sampling sites on the Tongue River or Powder River. m, meters; \%, percent; <, less than; mm, millimeters; $\mathrm{D}_{50}$, diameter of the 50 th percentile of particles; $\mathrm{D}_{84}$, diameter of the 84 th percentile of particles; $\mathrm{ft}^{3} / \mathrm{s}$, cubic feet per second; $\mu \mathrm{S} / \mathrm{cm}$, microsiemens per centimeter at $25 \mathrm{degrees}$ Celsius; ${ }^{\circ} \mathrm{C}$, degrees Celsius; mg/L, milligrams per liter; NTRU, nephelometric turbidity ratio units; NA, not applicable]

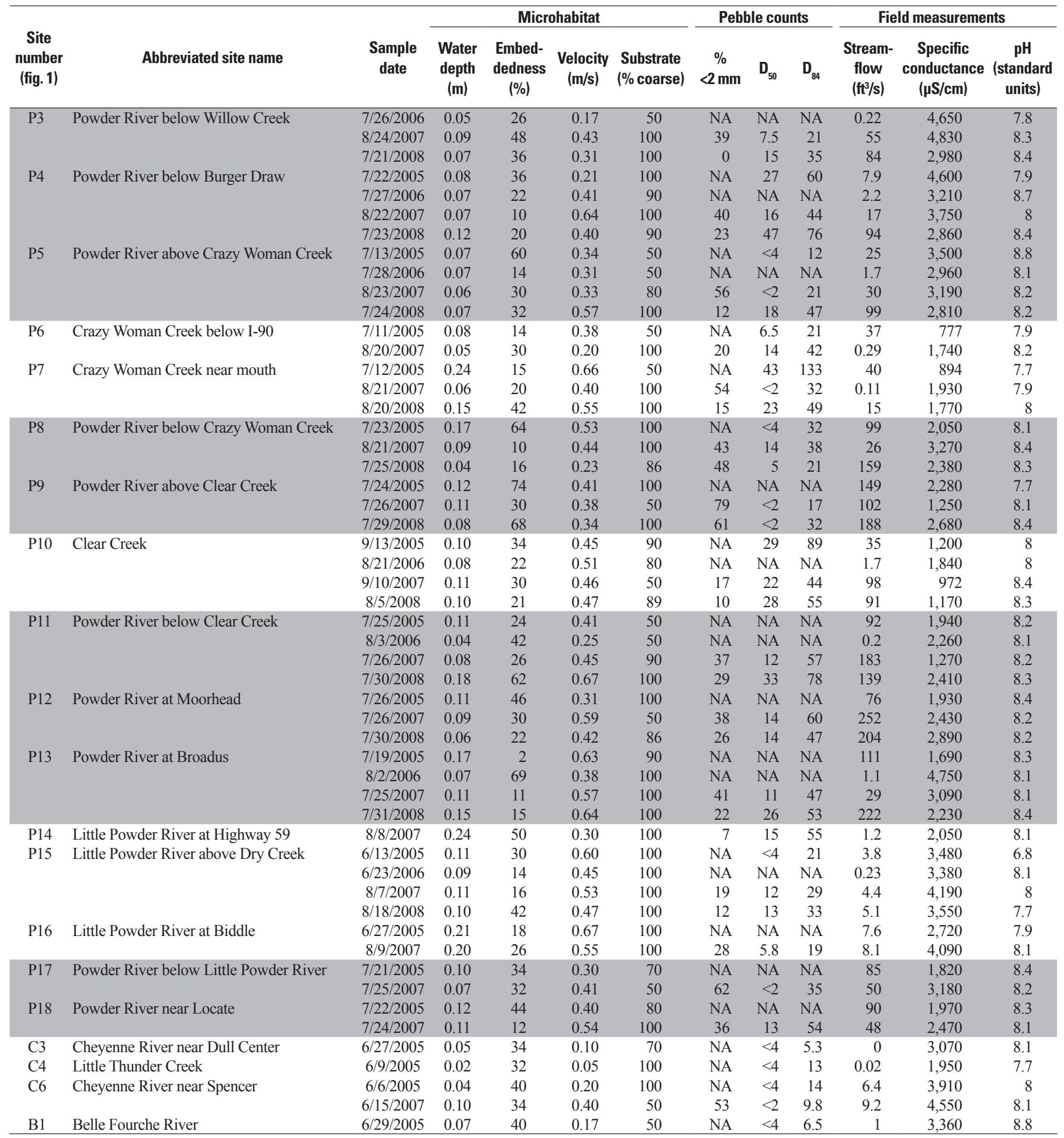


Table 3. Environmental variables associated with ecological samples, Powder River structural basin, Wyoming and Montana, 2005-08. - Continued

[Shaded cells indicate main-stem sampling sites on the Tongue River or Powder River. m, meters; \%, percent; <, less than; mm, millimeters; $\mathrm{D}_{50}$, diameter of the 50 th percentile of particles; $\mathrm{D}_{84}$, diameter of the 84 th percentile of particles; $\mathrm{ft}^{3} / \mathrm{s}$, cubic feet per second; $\mu \mathrm{S} / \mathrm{cm}$, microsiemens per centimeter at 25 degrees Celsius; ${ }^{\circ} \mathrm{C}$, degrees Celsius; $\mathrm{mg} / \mathrm{L}$, milligrams per liter; NTRU, nephelometric turbidity ratio units; NA, not applicable]

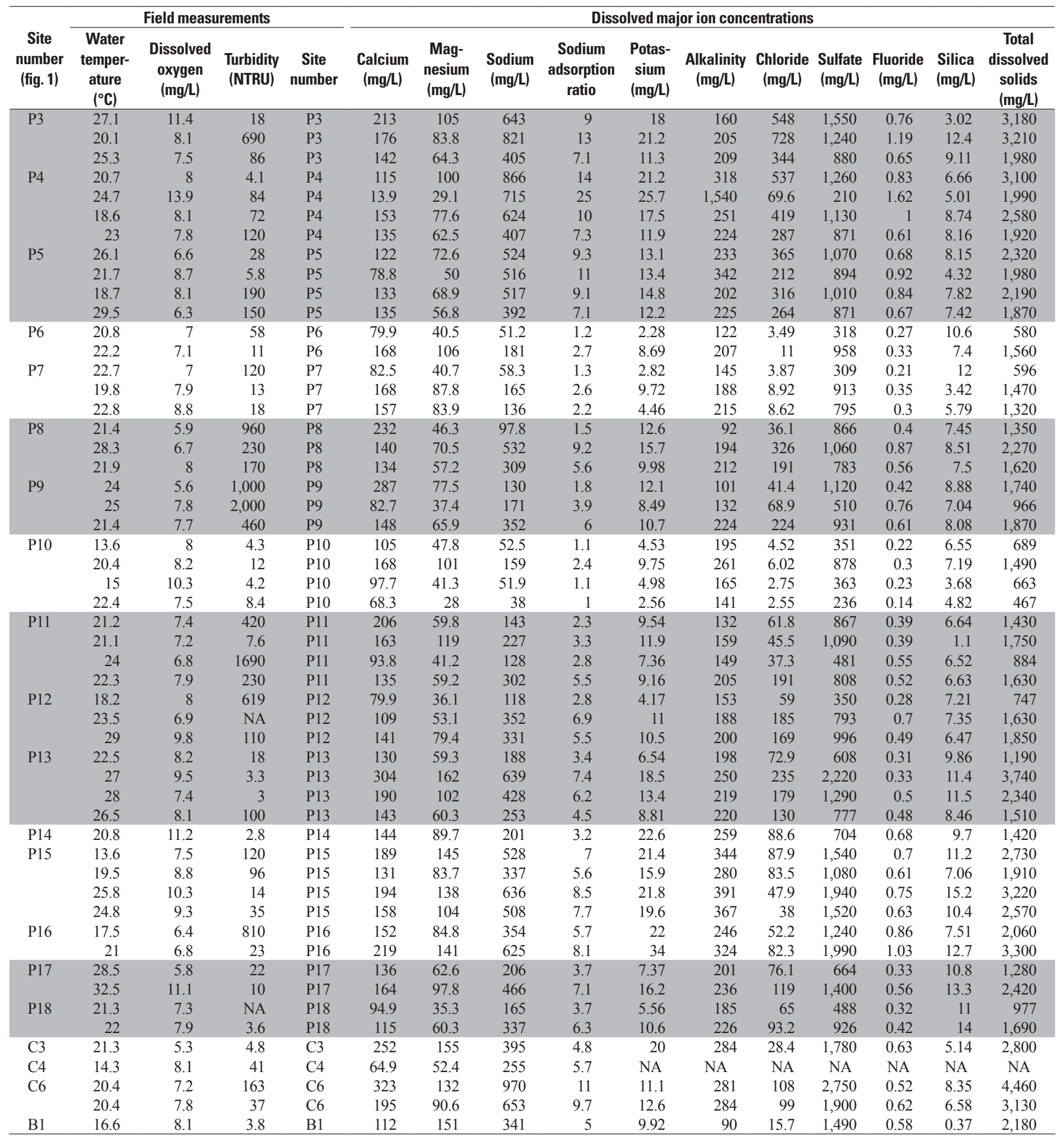



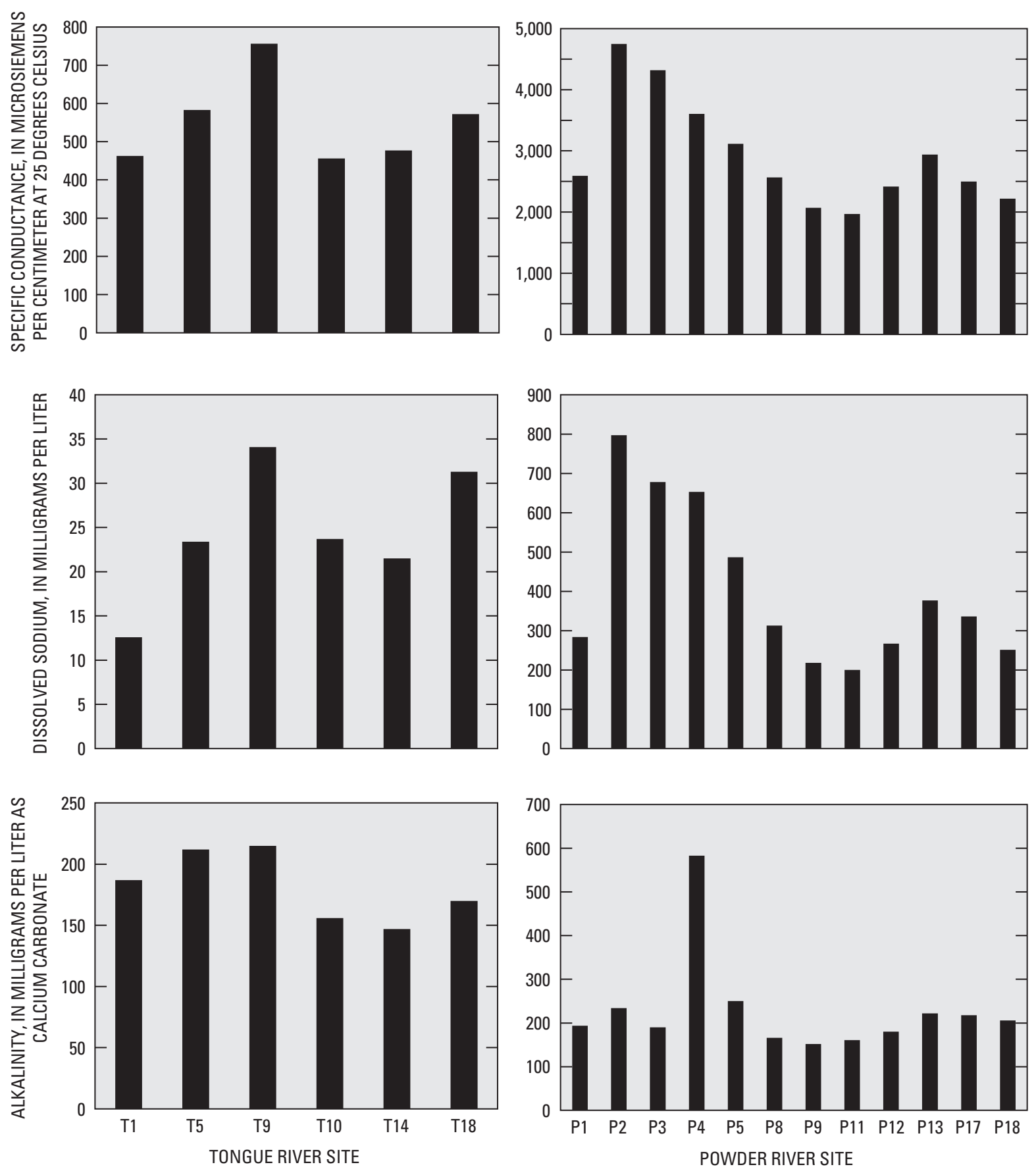

Figure 4. Mean values for specific conductance, dissolved sodium, and alkalinity for the Tongue River and Powder River during ecological sampling events, Wyoming and Montana, 2005-08.

Other water-quality constituents are classified by the USEPA as nonpriority pollutants, which do not require criteria to be established. Nonpriority pollutants evaluated for this report include $\mathrm{pH}$, chloride, aluminum, iron, and manganese. The USEPA has determined that results of toxicity and bioconcentration tests for total-chloride concentrations are essentially equivalent to results for dissolved-chloride concentrations (U.S. Environmental Protection Agency, 1988); therefore, dissolved-chloride concentrations were used for comparison in this report. The State of Wyoming has established acute and chronic chloride criteria for streams other than the Powder River downstream from Salt Creek. Because Salt Creek contributes high chloride concentrations to the Powder River, a site-specific criterion was established for the Powder River downstream from Salt Creek (Wyoming Department of Environmental Quality, 2007). 
Table 4. State and Federal criteria for the protection of aquatic life.

[State of Wyoming criteria are listed in Wyoming Department of Environmental Quality (2007); State of Montana criteria are listed in Montana Department of Environmental Quality (2008); Federal criteria are listed in U.S. Environmental Protection Agency (2005); --, not available; concentrations for all consitutents in micrograms per liter, unless otherwise noted; $\mathrm{mg} / \mathrm{L}$, milligrams per liter]

\begin{tabular}{|c|c|c|c|c|c|c|}
\hline \multirow[b]{2}{*}{ Constituent } & \multicolumn{2}{|c|}{ State of Wyoming } & \multicolumn{2}{|c|}{ State of Montana } & \multicolumn{2}{|c|}{ U.S. Environmental Protection Agency } \\
\hline & $\begin{array}{l}\text { Aquatic-life } \\
\text { acute value }\end{array}$ & $\begin{array}{c}\text { Aquatic-life } \\
\text { chronic value }\end{array}$ & $\begin{array}{l}\text { Aquatic-life } \\
\text { acute value }\end{array}$ & $\begin{array}{l}\text { Aquatic-life } \\
\text { chronic value }\end{array}$ & $\begin{array}{l}\text { Aquatic-life } \\
\text { acute value }\end{array}$ & $\begin{array}{l}\text { Aquatic-life } \\
\text { chronic value }\end{array}$ \\
\hline \multicolumn{7}{|c|}{ Priority pollutants } \\
\hline Arsenic, total & -- & -- & 340 & 150 & -- & -- \\
\hline Cadmium, dissolved & 12.0 & ${ }^{1} .25$ & -- & -- & 12.0 & ${ }^{1} .25$ \\
\hline Cadmium, total & -- & -- & ${ }^{1} 2.1$ & ${ }^{1} .27$ & -- & -- \\
\hline Chromium (VI), dissolved & 16 & 11 & -- & -- & 16 & 11 \\
\hline Chromium (VI), total & -- & -- & 16 & 11 & -- & -- \\
\hline Copper, dissolved & ${ }^{1} 13.4$ & 19.0 & -- & -- & 55.9 & -- \\
\hline Copper, total & -- & -- & ${ }^{1} 14$ & ${ }^{1} 9.3$ & -- & -- \\
\hline Lead, dissolved & ${ }^{1} 64.6$ & ${ }^{12.5}$ & -- & -- & ${ }^{1} 65$ & ${ }^{1} 2.5$ \\
\hline Nickel, total & -- & -- & ${ }^{1} 469$ & ${ }^{1} 52.2$ & -- & -- \\
\hline Selenium, dissolved & 20 & -- & -- & -- & -- & -- \\
\hline Selenium, total & -- & 5.0 & 20 & 5.0 & (6) & 5.0 \\
\hline Zinc, dissolved & ${ }^{1} 117.2$ & ${ }^{1} 118.1$ & -- & -- & ${ }^{1} 120$ & ${ }^{1} 120$ \\
\hline Zinc, total & -- & -- & ${ }^{1} 120$ & ${ }^{1} 120$ & -- & -- \\
\hline \multicolumn{7}{|c|}{ Nonpriority pollutants } \\
\hline $\mathrm{pH}$ (standard units) & -- & $6.5-9.0$ & -- & -- & -- & $6.5-9.0$ \\
\hline Chloride, total (mg/L) & ${ }^{2} 860,000$ & ${ }^{2} 230,000$ & -- & -- & 860,000 & 230,000 \\
\hline Chloride, total $(\mathrm{mg} / \mathrm{L})$ & ${ }^{3} 984,000$ & -- & -- & -- & -- & -- \\
\hline Aluminum, dissolved & 750 & ${ }^{4} 87$ & 750 & 87 & -- & -- \\
\hline Aluminum, total & -- & -- & -- & -- & 750 & 87 \\
\hline Iron, dissolved & -- & 1,000 & -- & -- & -- & -- \\
\hline
\end{tabular}

\section{State of Wyoming Aquatic-Life Criteria}

Water-quality constituents from 24 ATG study sites in Wyoming for water years 2005-08 were compared to State of Wyoming aquatic-life criteria (table 4). Concentrations of dissolved arsenic, dissolved cadmium, dissolved chromium (III), dissolved chromium (VI), dissolved copper, dissolved lead, total mercury, dissolved nickel, and dissolved zinc were less than aquatic-life acute and chronic criteria in all samples (table 5). Dissolved selenium concentrations were less than the acute criterion in all samples. Total selenium concentrations exceeded the chronic criterion in two samples collected from Caballo Creek (site B2) in the Belle Fourche River drainage basin.
A few samples had concentrations of nonpriority pollutants that were higher than aquatic-life criteria. One $\mathrm{pH}$ sample (9.8) for Porcupine Creek (site C1) in the Cheyenne River drainage basin was outside the chronic criterion range (table 5). Three samples from the Little Powder River above Dry Creek (site P15) had dissolved-chloride concentrations higher than the chronic criterion. Dissolved-chloride concentrations in all samples collected from sites on the Powder River downstream from Salt Creek were less than the sitespecific acute criterion for the Powder River. Dissolved-iron concentrations exceeded the chronic criterion in four samples from Antelope Creek (site C2) and seven samples from 
Table 5. Aquatic-life criteria comparison for water samples collected from study sites in the Powder River structural basin, Wyoming and Montana, water years 2005-08.

[number of samples, number of samples greater than criterion or standard/total number of samples collected; --, not applicable]

\begin{tabular}{|c|c|c|c|c|c|c|c|c|c|c|c|c|}
\hline \multirow{3}{*}{ Constituent } & \multicolumn{4}{|c|}{ State of Wyoming } & \multicolumn{4}{|c|}{ State of Montana } & \multicolumn{4}{|c|}{ U.S. Environmental Protection Agency } \\
\hline & \multicolumn{2}{|c|}{ Acute } & \multicolumn{2}{|c|}{ Chronic } & \multicolumn{2}{|c|}{ Acute } & \multicolumn{2}{|c|}{ Chronic } & \multicolumn{2}{|c|}{ Acute } & \multicolumn{2}{|c|}{ Chronic } \\
\hline & $\begin{array}{c}\text { Number of } \\
\text { samples }\end{array}$ & $\begin{array}{c}\text { Sites with } \\
\text { exceedances }\end{array}$ & $\begin{array}{c}\text { Number of } \\
\text { samples }\end{array}$ & $\begin{array}{c}\text { Sites with } \\
\text { exceedances }\end{array}$ & $\begin{array}{c}\text { Number of } \\
\text { samples }\end{array}$ & $\begin{array}{c}\text { Sites with } \\
\text { exceedances }\end{array}$ & $\begin{array}{c}\text { Number of } \\
\text { samples }\end{array}$ & $\begin{array}{c}\text { Sites with } \\
\text { exceedances }\end{array}$ & $\begin{array}{c}\text { Number of } \\
\text { samples }\end{array}$ & $\begin{array}{c}\text { Sites with } \\
\text { exceedances }\end{array}$ & $\begin{array}{c}\text { Number of } \\
\text { samples }\end{array}$ & $\begin{array}{c}\text { Sites with } \\
\text { exceedances }\end{array}$ \\
\hline Arsenic, dissolved & $0 / 380$ & -- & $0 / 380$ & -- & -- & --- & -- & -- & $0 / 620$ & -- & $0 / 620$ & -- \\
\hline Arsenic, total & -- & -- & -- & -- & $0 / 253$ & -- & $0 / 253$ & -- & -- & -- & -- & -- \\
\hline Cadmium, dissolved & $0 / 46$ & -- & $0 / 46$ & -- & -- & -- & -- & -- & $0 / 153$ & -- & $0 / 153$ & -- \\
\hline Cadmium, total & -- & -- & -- & -- & $6 / 207$ & T19, P18 & $24 / 207$ & T19, P12, P18 & -- & -- & -- & -- \\
\hline Chromium (III), dissolved & $0 / 3$ & -- & $0 / 3$ & -- & -- & -- & -- & -- & $0 / 35$ & -- & $0 / 35$ & -- \\
\hline Chromium (III), total & -- & -- & -- & -- & $0 / 207$ & -- & $4 / 207$ & T19, P18 & -- & -- & -- & -- \\
\hline Chromium (VI), dissolved & $0 / 3$ & -- & $0 / 3$ & -- & -- & -- & -- & -- & $0 / 35$ & -- & $0 / 35$ & -- \\
\hline Chromium (VI), total & -- & -- & -- & -- & $28 / 207$ & T19, P12, P18 & $36 / 207$ & T19, P12, P18 & -- & -- & -- & -- \\
\hline Copper, dissolved & $0 / 43$ & -- & $0 / 43$ & -- & -- & -- & -- & -- & $5 / 150$ & T19 & -- & -- \\
\hline Copper, total & -- & -- & -- & -- & $24 / 207$ & T19, P12, P18 & $35 / 207$ & $\begin{array}{c}\text { T9, T19, P12, } \\
\text { P18 }\end{array}$ & -- & -- & -- & -- \\
\hline Lead, dissolved & $0 / 44$ & -- & $0 / 44$ & -- & -- & -- & -- & -- & $0 / 151$ & -- & $1 / 151$ & T19 \\
\hline Lead, total & -- & -- & -- & -- & $7 / 207$ & T19, P18 & $36 / 207$ & $\begin{array}{l}\text { T9, T19, P12, } \\
\text { P18 }\end{array}$ & -- & -- & -- & -- \\
\hline Mercury, total & $0 / 24$ & -- & $0 / 24$ & -- & $0 / 55$ & -- & $0 / 55$ & -- & $0 / 79$ & -- & $0 / 79$ & -- \\
\hline Nickel, dissolved & $0 / 44$ & -- & $0 / 44$ & -- & -- & -- & -- & -- & $0 / 151$ & -- & $0 / 151$ & -- \\
\hline Nickel, total & -- & -- & -- & -- & 2/207 & T19 & $8 / 207$ & T19, P18 & -- & -- & -- & -- \\
\hline Selenium, dissolved & $0 / 52$ & -- & -- & -- & -- & -- & -- & -- & -- & -- & -- & -- \\
\hline Selenium, total & -- & -- & $2 / 382$ & B2 & $0 / 289$ & -- & $1 / 289$ & $\mathrm{P} 12$ & -- & -- & $3 / 671$ & P12, B2 \\
\hline Zinc, dissolved & $0 / 43$ & -- & $0 / 43$ & -- & -- & -- & -- & -- & $0 / 144$ & -- & $0 / 144$ & -- \\
\hline Zinc, total & -- & -- & -- & -- & $11 / 207$ & T19, P12, P18 & $11 / 207$ & T19, P12, P18 & -- & -- & -- & -- \\
\hline $\mathrm{pH}$ & $1 / 517$ & $\mathrm{C} 1$ & -- & -- & -- & -- & -- & -- & $3 / 1,045$ & $\mathrm{~T} 11, \mathrm{~T} 12, \mathrm{C} 1$ & -- & -- \\
\hline Chloride, dissolved & $0 / 450$ & -- & $3 / 450$ & P15 & -- & -- & -- & -- & $2 / 1,001$ & P2 & $20 / 1,001$ & $\begin{array}{c}\text { P2, P3, P4, } \\
\text { P5, P8, P12, } \\
\text { P13,P15 }\end{array}$ \\
\hline $\begin{array}{l}\text { Chloride, dissolved } \\
\text { (Powder River) }\end{array}$ & $0 / 27$ & -- & -- & -- & -- & -- & -- & -- & -- & -- & -- & -- \\
\hline Aluminum, dissolved & $0 / 52$ & -- & -- & -- & $0 / 136$ & -- & $0 / 136$ & -- & -- & -- & -- & -- \\
\hline Aluminum, total & -- & -- & -- & -- & -- & -- & -- & -- & $160 / 623$ & $\begin{array}{c}\text { R1, R2, T1, T2, } \\
\text { T8, T9, T14, } \\
\text { T17, T18, T19, } \\
\text { P10, P12, P15, } \\
\text { C1, C3, C5, } \\
\text { C6, B1 }\end{array}$ & $390 / 623$ & $\begin{array}{c}\text { R1, R2, T1, T2, } \\
\text { T8, T9, T13, } \\
\text { T14, T17, T18, } \\
\text { T19, P10, P12, } \\
\text { P15, C1, C2, } \\
\text { C3, C5, C6, } \\
\text { B1, B2 }\end{array}$ \\
\hline Iron, dissolved & -- & -- & $11 / 380$ & $\mathrm{C} 2, \mathrm{C} 3$ & -- & -- & -- & -- & -- & -- & -- & -- \\
\hline Iron, total & -- & -- & -- & -- & -- & -- & $111 / 232$ & $\begin{array}{l}\text { R1, R2, T9, T14, } \\
\text { T17, T18, T19, } \\
\text { P12, P18 }\end{array}$ & -- & -- & $140 / 331$ & $\begin{array}{c}\text { R1, R2, T1, T2, } \\
\text { T8, T9, T14, } \\
\text { T17, T18, T19, } \\
\text { P12, P15, P18 }\end{array}$ \\
\hline Manganese, dissolved & $3 / 380$ & $\mathrm{C} 2, \mathrm{~B} 1$ & $16 / 380$ & $\mathrm{C} 2, \mathrm{C} 3, \mathrm{C} 6, \mathrm{~B} 1$ & -- & -- & -- & -- & -- & -- & -- & -- \\
\hline
\end{tabular}


the Cheyenne River near Dull Center (site C3). Dissolvedmanganese concentrations exceeded the acute criterion in two samples from Antelope Creek (site C2) and one sample from the Belle Fourche River (site B1). Dissolved-manganese concentrations exceeded the chronic criterion in 10 samples from Antelope Creek (site C2), 3 samples from the Cheyenne River near Dull Center (site C3), 1 sample from the Cheyenne River near Spencer (site C6), and 2 samples from the Belle Fourche River (site B1).

\section{State of Montana Aquatic-Life Criteria}

Water-quality constituents from 23 ATG study sites in Montana for water years 2005-08 were compared to State of Montana aquatic-life criteria (table 4). Concentrations of total arsenic and total mercury were less than aquatic-life acute and chronic criteria in all samples (table 5).

Concentrations of other priority pollutants exceeded aquaticlife criteria in some samples. Total-cadmium concentrations exceeded acute criteria in four samples from Pumpkin Creek in the Tongue River drainage basin (site T19) and two samples from the Powder River near Locate (site P18). Total-cadmium concentrations exceeded chronic criteria that were calculated for seven samples from Pumpkin Creek (site T19); eight samples from the Powder River at Moorhead (site P12); and nine samples from the Powder River near Locate (site P18).

No samples had concentrations of chromium (III) higher than the applicable aquatic-life acute criteria (table 5). Totalchromium concentrations exceeded chromium (III) chronic criteria in three samples from Pumpkin Creek (site T19) and one sample from the Powder River near Locate (site P18). Totalchromium concentrations exceeded the chromium (VI) acute criterion in 5 samples from Pumpkin Creek (site T19), 10 samples from the Powder River at Moorhead (site P12), and 13 samples from the Powder River near Locate (site P18). Total-chromium concentrations exceeded the chromium (VI) chronic criterion in 5 samples from Pumpkin Creek (site T19), 12 samples from the Powder River at Moorhead (site P12), and 19 samples from the Powder River near Locate (site P18); however, these comparisons assume that all of the chromium was present in the +6 valence state, which would not be typical for stream conditions.

Total-copper concentrations exceeded aquatic-life acute criteria in seven samples from Pumpkin Creek (site T19), eight samples from the Powder River at Moorhead (site P12), and nine samples from the Powder River near Locate (site P18, table 5). Total-copper concentrations exceeded chronic criteria in 1 sample from the Tongue River at State line (site T9), 8 samples from Pumpkin Creek (site T19), 9 samples from the Powder River at Moorhead (site P12), and 17 samples from the Powder River near Locate (site P18).

Total-lead concentrations exceeded aquatic-life acute criteria that were calculated for samples in four samples from Pumpkin Creek (site T19) and three samples from the Powder River near Locate (site P18). Total-lead concentrations exceeded chronic criteria in 2 samples from the Tongue River at State line (site T9), 7 samples from Pumpkin Creek (site T19), 10 samples from the Powder River at Moorhead (site P12), and 17 samples from the Powder River near Locate (site P18).

Total-nickel concentrations exceeded acute criteria in two samples from Pumpkin Creek (site T19). Total-nickel concentrations exceeded chronic criteria that were calculated for samples in five samples from Pumpkin Creek (site T19) and three samples from the Powder River near Locate (site P18). No samples had a total-selenium concentration higher than the aquatic-life acute criterion. The total-selenium concentration exceeded the chronic criterion in one sample from the Powder River at Moorhead (site P12). Total-zinc concentrations exceeded aquatic-life acute and chronic criteria in six samples from Pumpkin Creek (site T19), one sample from the Powder River at Moorhead (site P12), and four samples from the Powder River near Locate (site P18).

For nonpriority pollutants, no samples had dissolvedaluminum concentrations higher than aquatic-life acute or chronic criteria. Total-iron concentrations exceeded the chronic criterion in 1 sample from upper Rosebud Creek (site R1), 6 samples from Rosebud Creek at mouth (site R2), 4 samples from the Tongue River at State line (site T9), 3 samples from the Tongue River at Birney Day School (site T14), 7 samples from Otter Creek at mouth (site T17), 2 samples from the Tongue River below Brandenberg Bridge (site T18), 8 samples from Pumpkin Creek (site T19), 40 samples from the Powder River at Moorhead (site P12), and 40 samples from the Powder River near Locate (site P18) (table 5).

\section{Federal Aquatic-Life Criteria}

Concentrations of dissolved arsenic, dissolved cadmium, dissolved chromium (III), dissolved chromium (VI), total mercury, dissolved nickel, and dissolved zinc were less than USEPA national recommended aquatic-life acute and chronic criteria in all samples (table 5). Five samples from Pumpkin Creek (site T19), with low hardness values, exceeded the applicable dissolved-copper acute criterion of $5.9 \mu \mathrm{g} / \mathrm{L}$. Dissolved-lead concentrations in all samples were less than the USEPA national recommended aquatic-life acute and chronic criteria except for one sample from Pumpkin Creek (site T19) that exceeded the chronic criterion. Total-selenium concentrations exceeded the chronic criterion in one sample from the Powder River at Moorhead (site P12) and two samples from Caballo Creek (site B2).

Some samples had concentrations of nonpriority pollutants that exceeded aquatic-life criteria (table 5). Three measurements of $\mathrm{pH}$, one at each of the upper Hanging Woman Creek (site T11), middle Hanging Woman Creek (site T12), and Porcupine Creek (site C1) sites, exceeded 9.0 standard units. Dissolved-chloride concentrations exceeded the acute criterion in two samples from Powder River below Salt Creek (P2). Dissolved-chloride concentrations exceeded the chronic criterion in three samples from the Powder River below Salt Creek (P2), four samples from the Powder River above 
Pumpkin Creek (site P3), three samples from the Powder River below Burger Draw (site P4), three samples from the Powder River above Crazy Woman Creek (site P5), two samples from the Powder River below Crazy Woman Creek (P8), one sample from the Powder River at Moorhead (site P12), one sample from the Powder River at Broadus (site P13), and three samples from the Little Powder River above Dry Creek (site P15). Unlike the States of Wyoming and Montana, the USEPA national recommended aquatic-life criteria for aluminum are based on total concentrations. Total-aluminum concentrations exceeded the chronic criterion, acute criterion, or both (table 5) at all 21 sites that had analyses for total aluminum (sites R1, R2, T1, T2, T8, T9, T13, T14, T17, T18, T19, P10, P12, P15, C1, C2, C3, C5, C6, B1, and B2). Totaliron concentrations exceeded the chronic criterion at 13 of the 14 sites that had analyses for total iron (sites R1, R2, T1, T2, T8, T9, T14, T17, T18, T19, P12, P15, and P18).

\section{Habitat}

Among all sites, embeddedness of the substrate, measured in conjunction with macroinvertebrate sampling in riffles during 2005-08, ranged from 0 to 74 percent (table 3 ). The values for embeddedness and other microhabitat datawater depth, velocity, and coarse substrate (fraction of gravel or larger size particles) - listed in table 3 represent mean values from five points accompanying each macroinvertebrate sample. Among all sites, microhabitat substrate ranged from 50 percent to 100 percent coarse substrate during 2005-08.

Pebble counts indicated a wide variation in particle size percentiles among sites during 2007-08. For example, the $\mathrm{D}_{84}$, or diameter of the 84 th percentile of particles, ranged from 5.3 to 138 millimeters (mm) (table 3). Pebble-count data for the $\mathrm{D}_{50}$, or diameter of the 50th percentile (median) of particles, and the percentage less than $2 \mathrm{~mm}$ (smallest size measured) also are listed in table 3 . The pebble-count data were collected from transects bankfull to bankfull across riffles according to methods by Wolman (1954) and therefore represent a slightly different aspect of the riffles than the microhabitat substrate measurements that were collected at an average depth of 0.4 feet ( $\mathrm{ft}$; 0.11 meter) in riffles according to methods by Moulton and others (2002). The microhabitat and pebble-count data were collected as indicators of physical variables potentially affecting macroinvertebrate communities.

\section{Macroinvertebrate Communities}

Macroinvertebrate taxonomic data from 2005-08 are available at: http://wy.water.usgs.gov/projects/atg/htms/ data.htm. Most of the taxa identified were Chironomidae (midges:Diptera), Ephemeroptera (mayflies), and Trichoptera (caddisflies). Other identified taxa, though generally rarer, included Coleoptera (beetles), noninsects (such as worms: Oligochaeta, and snails:Gastropoda), Odonata (dragonflies and damselflies), and Plecoptera (stoneflies). The total number of taxa (taxa richness) in the ATG samples ranged from 5 taxa in Pumpkin Creek (site T19, 2005) to 47 taxa in Hanging Woman Creek (site T11, 2005) (table 6).

\section{Macroinvertebrate Community Composition}

Three stream groups of sites with relatively similar taxonomic composition were identified: Tongue River main stem and mountainous tributaries (TRMS), Tongue River plains tributaries (TRPT), and Powder River main stem (PRMS). NMDS ordinations for 2007-08 (fig. 5) and 2005-06 (Peterson and others, 2009) indicated the same general stream groups for all 4 years of sampling, with a few sites that were sometimes outliers. The TRMS group generally contained sites sampled on the main-stem Tongue River (sites T1, T5, T9, T10, T14, and T18) and tributaries originating in the Bighorn Mountains: Goose Creek (site T2), Crazy Woman Creek (sites P6 and P7), and Clear Creek (site P10). The TRPT group generally contained sites on Youngs Creek (sites T3 and T4), Squirrel Creek (site T7), Prairie Dog Creek (site T8), Hanging Woman Creek (sites T11-T13), Otter Creek (sites T16 and T17), and Pumpkin Creek (site T19). Prairie Dog Creek (site T8) plotted with the TRMS group although the headwaters are in the plains (table 1). The PRMS group generally contained sites P1-P5, P8, P9, P11-P13, P17, and P18. Sampling sites on Rosebud Creek (site R2), the Little Powder River (sites P14-P16), the Cheyenne River drainage basin (sites C3, C4, and C6), and the Belle Fourche River (site B1) tended to be outliers to the stream groups. Some of the sites, particularly the outliers, are not shown in figure 5 because they were sampled only in 2005 or 2006 (Peterson and others, 2009).

Taxa richness at sites in the TRMS group ranged from 17 to 41 taxa per sample during 2005-08 (table 6), with a mean of 29 taxa per sample. Diptera, Ephemeroptera, and Trichoptera dominated in terms of taxa richness, although on the average, relative abundance was dominated by Ephemeroptera (46 percent), Trichoptera (21 percent), and Diptera (12 percent). Ephemeroptera taxa richness and relative abundance of Ephemeroptera individuals tended to be higher at sites in the TRMS group than in the TRPT or PRMS groups (fig. 6). The Ephemeroptera Baetis, Fallceon quilleri, and Tricorythodes were the most abundant macroinvertebrates in the TRMS group. The most common functional feeding mode at sites in the TRMS group was the collector-gatherers (mean of 59 percent), with smaller proportions of filter-collectors (mean of 26 percent) and scrapers (mean of 9 percent). Other functional feeding modes such as predators and omnivores accounted for the remainder. The proportion of intolerant macroinvertebrates averaged 53 percent and generally was higher in the TRMS group than the TRPT or PRMS groups 
(fig. 6). The 2007 sample from the main-stem Tongue River above Hanging Woman Creek (site T10) was an outlier to the TRMS group in the ordination (fig. 5) because the community was composed primarily of noninsects and Diptera, such as the snail Physa, the scud Hyallela, and the blackfly Simulium that are tolerant organisms (2 percent intolerant, table 6). Site T10 plotted within ordinations of the TRMS group during 2005-06 (Peterson and others, 2009) and 2008. The macroinvertebrate communities at site T10 might be affected by Tongue River Reservoir upstream as noted in the section "Spatial and Temporal Patterns."

Taxa richness at sites in the TRPT group was dominated by Diptera, with a mean of 16 Diptera taxa compared to a mean of 30 taxa total per sample during 2005-08. The Diptera also predominated in terms of relative abundance (table 6), accounting for an average of 52 percent of the macroinvertebrates. Although most of the Diptera taxa were Chironomidae, the blackfly larvae Simulium were common and sometimes numerically dominant. Trichoptera larvae, such as Cheumatopsyche and Hydropsyche, also were abundant in some of the TRPT samples. Macroinvertebrate communities in the TRPT group were characterized by distinctively lower relative abundance of intolerant macroinvertebrates than the TRMS or PRMS groups (fig. 6).

Taxa richness in the PRMS group ranged from 9 to 38 taxa and averaged 22 taxa per sample. Taxa richness in the PRMS group tended to be lower than in the TRMS or TRPT groups (fig. 6). Diptera dominated the taxa richness (average of 9 taxa per sample) and relative abundance (average of 45 percent per sample) of the PRMS group. The proportion of filterer-collectors was relatively high in the PRMS group, due in part to the dominance of the blackfly larvae Simulium in many of the samples. Macroinvertebrate communities in the PRMS group tended to be intermediate to those of the TRMS and TRPT groups in terms of Ephemeroptera taxa richness, relative abundance of Ephemeroptera, and relative abundance of intolerant macroinvertebrates.

Taxa richness in macroinvertebrate communities of the Little Powder River (sites P14-P16) was dominated by Diptera, whereas Trichoptera predominated in terms of relative abundance (table 6). Communities of the Little Powder River also were characterized by high relative abundance of filterercollectors and low relative abundance of intolerant macroinvertebrates. Communities at sites on Rosebud Creek (sites R1 and R2) and in the Cheyenne (sites C1-C6) and Belle Fourche River (B1 and B2) drainage basins were characterized by low taxa richness, high proportions of filterer-collectors, and low proportions of intolerant macroinvertebrates; Diptera and noninsects often predominated in terms of taxa richness and relative abundance (table 6). Intermittent flow conditions, such as in the Cheyenne and Belle Fourche River drainage basins, and high specific-conductance values and flooding common to plains streams (Wangsness and Peterson, 1980) create a harsh environment for macroinvertebrate communities, regardless of anthropogenic effects.

\section{Spatial and Temporal Patterns}

Data from the main-stem Tongue River and main-stem Powder River were analyzed using ANOVA to examine spatial patterns, such as differences by site in the downstream direction, and temporal patterns, which were differences by year. A brief discussion of spatial and temporal patterns at sites on tributaries also is presented, but the tributaries did not have sufficient data for analysis using ANOVA. Therefore, the site groupings in this section are slightly different from the groupings used to describe macroinvertebrate community composition.

\section{Main-Stem Tongue River}

Results of ANOVA using the Tukey method for analysis of metric data from main-stem Tongue River sites T1, T5, T9, T10, T14, and T18 are listed in table 7. Macroinvertebrate community metrics did not vary significantly among main-stem Tongue River sites, with one exception. During 2005-08, the relative abundance of noninsects in the Tongue River above Hanging Woman Creek (site T10) was significantly different $(\mathrm{p}<0.05)$ from that of the Tongue River at sites T9, T14, and T18. As shown in figure 7 , the mean relative abundance of noninsects during 2005-08 was higher at site T10 (about 16 percent) than at other sites on the main-stem Tongue River (4 percent or less). The macroinvertebrate communities at site T10 might be affected by Tongue River Reservoir, which is on the main-stem Tongue River about 9 air-miles upstream from site T10. The mean relative abundance of Trichoptera decreased from site T1 to site T9 (fig. 7).

The ANOVA also indicated significant variation $(p<0.05)$ between years in the relative abundance of macroinvertebrates in the scraper feeding mode (table 7). The mean relative abundance of scrapers was higher in 2006 than other years (fig. 7). Though not significant at $\mathrm{p}<0.05$, the proportion of intolerant macroinvertebrates also was higher in 2006 (mean 72 percent) than in other years (mean 44-52 percent; fig. 7). The extended drought that was particularly severe in 2006 might be at least partly responsible for year-to-year differences in the metric values.

\section{Main-Stem Powder River}

Macroinvertebrate community metrics on the mainstem Powder River indicated differences at sites in the middle reach from other sites on the main-stem Powder River. The ANOVA tests indicated one or more metrics varied significantly $(\mathrm{p}<0.05)$ at sites P3-P5 and P8 (the middle reach, from below the Willow Creek to below the Crazy Woman Creek confluences) from metrics at sites P9, P11, P12, P13, and P18 (the lower reach) from above the confluence with Clear Creek to Locate, Mont.; table 7). Graphical presentation of the mean values during 2005-08 for five metrics (fig. 8) shows that values from the middle reach (sites P3-P5 and P8) and to a lesser extent, site P2, were notably different than values at other sites on the main-stem Powder River both upstream (site P1) and 
Table 6. Macroinvertebrate community metrics for streams in the Powder River structural basin, Wyoming and Montana, 2005-08.

[Shaded cells indicate main-stem sampling sites on the Tongue or Powder River. \%, percent]

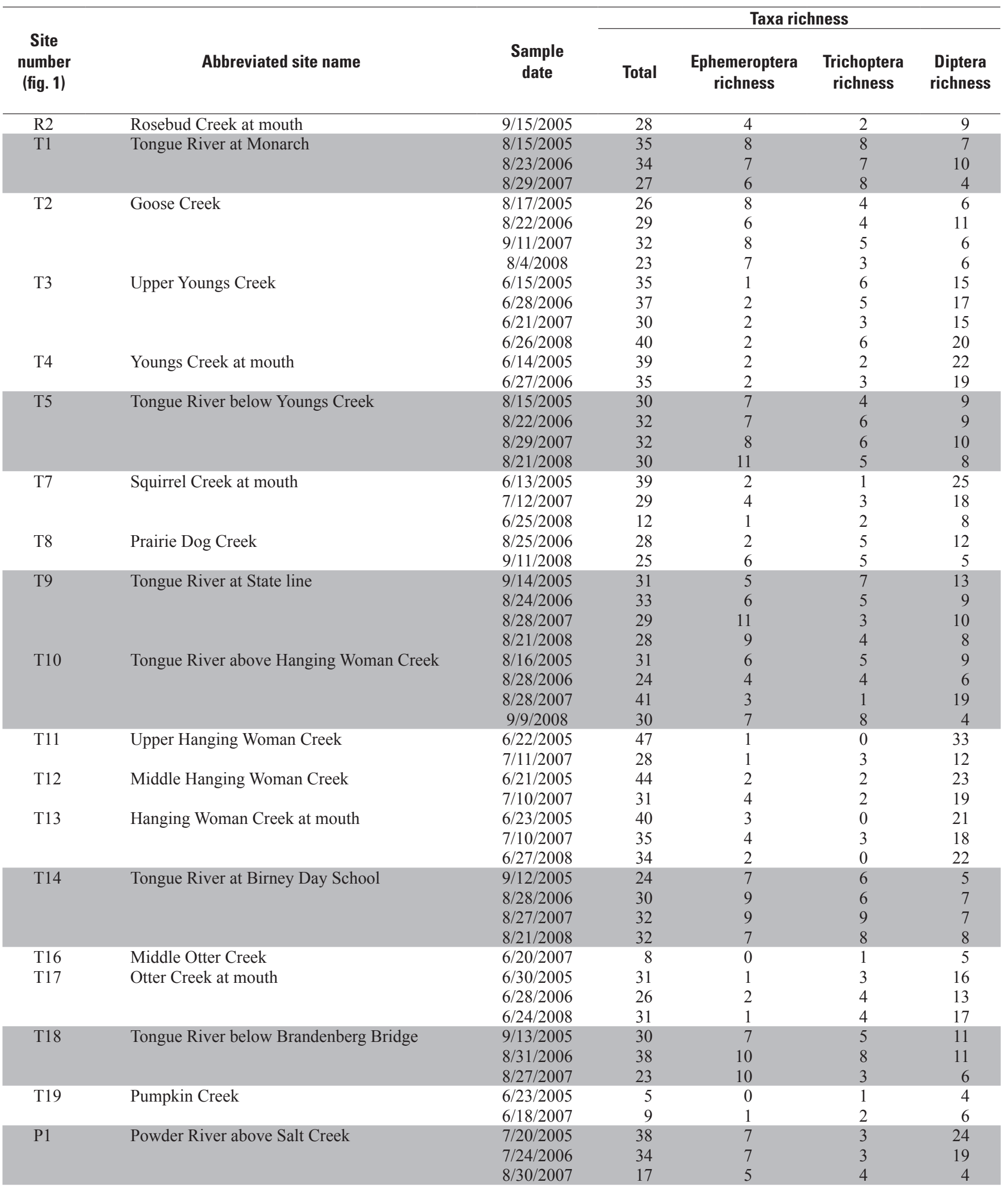


Table 6. Macroinvertebrate community metrics for streams in the Powder River structural basin, Wyoming and Montana, 2005-08. - Continued

[Shaded cells indicate main-stem sampling sites on the Tongue or Powder River. \%, percent]

\begin{tabular}{|c|c|c|c|c|c|c|c|c|}
\hline \multirow[b]{2}{*}{$\begin{array}{c}\text { Site } \\
\text { number } \\
\text { (fig. 1) }\end{array}$} & \multicolumn{4}{|c|}{ Taxa relative abundance } & \multicolumn{3}{|c|}{ Functional feeding mode relative abundance } & \multirow{2}{*}{$\begin{array}{c}\text { Tolerance } \\
\text { Intolerant } \\
\text { macro- } \\
\text { invertebrates } \\
(\%)\end{array}$} \\
\hline & $\begin{array}{c}\text { Ephemeroptera } \\
\text { abundance } \\
(\%)\end{array}$ & $\begin{array}{c}\text { Trichoptera } \\
\text { abundance } \\
(\%)\end{array}$ & $\begin{array}{c}\text { Diptera } \\
\text { abundance } \\
(\%)\end{array}$ & $\begin{array}{c}\text { Noninsect } \\
\text { abundance } \\
(\%)\end{array}$ & $\begin{array}{c}\text { Collector- } \\
\text { gatherer } \\
\text { abundance } \\
(\%)\end{array}$ & $\begin{array}{c}\text { Filterer- } \\
\text { collector } \\
\text { abundance } \\
(\%)\end{array}$ & $\begin{array}{c}\text { Scraper } \\
\text { abundance } \\
(\%)\end{array}$ & \\
\hline R2 & 2 & 19 & 15 & 14 & 23 & 19 & 39 & 4 \\
\hline \multirow[t]{3}{*}{$\mathrm{T} 1$} & 76 & 14 & 2 & 3 & 79 & 12 & 4 & 26 \\
\hline & 32 & 33 & 10 & 3 & 51 & 12 & 26 & 49 \\
\hline & 53 & 39 & 4 & 2 & 49 & 39 & 9 & 41 \\
\hline \multirow[t]{4}{*}{$\mathrm{T} 2$} & 57 & 16 & 2 & 10 & 72 & 15 & 5 & 41 \\
\hline & 42 & 7 & 7 & 1 & 75 & 6 & 12 & 47 \\
\hline & 54 & 29 & 5 & 4 & 57 & 29 & 8 & 56 \\
\hline & 50 & 29 & 17 & 1 & 51 & 44 & 2 & 38 \\
\hline \multirow[t]{4}{*}{ T3 } & 34 & 3 & 47 & 11 & 44 & 50 & 3 & 7 \\
\hline & 37 & 33 & 9 & 5 & 58 & 34 & 5 & 31 \\
\hline & 37 & 5 & 44 & 5 & 52 & 39 & 0 & 9 \\
\hline & 29 & 30 & 23 & 10 & 45 & 34 & 7 & 30 \\
\hline \multirow[t]{2}{*}{$\mathrm{T} 4$} & 12 & 7 & 30 & 41 & 57 & 19 & 10 & 1 \\
\hline & 6 & 15 & 27 & 45 & 52 & 30 & 11 & 1 \\
\hline \multirow[t]{4}{*}{ T5 } & 44 & 30 & 5 & 2 & 62 & 31 & 4 & 54 \\
\hline & 25 & 17 & 6 & 12 & 61 & 15 & 18 & 69 \\
\hline & 41 & 24 & 7 & 0 & 63 & 24 & 6 & 70 \\
\hline & 68 & 14 & 13 & 1 & 69 & 21 & 4 & 48 \\
\hline \multirow[t]{3}{*}{$\mathrm{T} 7$} & 1 & 1 & 79 & 15 & 59 & 25 & 4 & 0 \\
\hline & 2 & 4 & 85 & 9 & 4 & 64 & 1 & 4 \\
\hline & 4 & 1 & 95 & 0 & 4 & 95 & 0 & 0 \\
\hline \multirow[t]{2}{*}{ T8 } & 20 & 32 & 10 & 0 & 48 & 35 & 13 & 56 \\
\hline & 7 & 79 & 2 & 1 & 15 & 80 & 2 & 87 \\
\hline \multirow[t]{4}{*}{ T9 } & 69 & 14 & 12 & 0 & 74 & 17 & 3 & 28 \\
\hline & 39 & 13 & 4 & 9 & 71 & 11 & 7 & 79 \\
\hline & 85 & 3 & 11 & 1 & 78 & 5 & 10 & 43 \\
\hline & 64 & 7 & 10 & 0 & 78 & 8 & 8 & 51 \\
\hline \multirow[t]{4}{*}{$\mathrm{T} 10$} & 26 & 19 & 17 & 12 & 56 & 15 & 19 & 38 \\
\hline & 7 & 10 & 3 & 9 & 77 & 12 & 10 & 85 \\
\hline & 11 & 0 & 36 & 36 & 38 & 21 & 20 & 2 \\
\hline & 60 & 12 & 17 & 7 & 64 & 24 & 4 & 53 \\
\hline \multirow{2}{*}{ T11 } & 0 & 0 & 65 & 24 & 58 & 4 & 19 & 0 \\
\hline & 47 & 4 & 25 & 22 & 71 & 19 & 2 & 2 \\
\hline \multirow[t]{2}{*}{$\mathrm{T} 12$} & 4 & 4 & 36 & 48 & 40 & 7 & 29 & 2 \\
\hline & 9 & 32 & 42 & 16 & 20 & 36 & 8 & 35 \\
\hline \multirow[t]{3}{*}{$\mathrm{T} 13$} & 2 & 0 & 32 & 56 & 38 & 12 & 31 & 1 \\
\hline & 6 & 3 & 75 & 6 & 29 & 25 & 5 & 12 \\
\hline & 8 & 0 & 68 & 19 & 22 & 47 & 18 & 0 \\
\hline \multirow{4}{*}{ T14 } & 67 & 26 & 1 & 0 & 66 & 26 & 7 & 72 \\
\hline & 46 & 36 & 3 & 0 & 48 & 37 & 12 & 82 \\
\hline & 63 & 17 & 11 & 1 & 68 & 17 & 12 & 74 \\
\hline & 34 & 28 & 21 & 1 & 51 & 40 & 3 & 50 \\
\hline T16 & 0 & 0 & 100 & 0 & 1 & 99 & 0 & 0 \\
\hline T17 & 1 & 51 & 41 & 3 & 9 & 79 & 3 & 3 \\
\hline & 0 & 23 & 69 & 7 & 7 & 90 & 1 & 11 \\
\hline & 0 & 48 & 42 & 4 & 11 & 77 & 5 & 35 \\
\hline T18 & 39 & 37 & 16 & 0 & 40 & 38 & 7 & 48 \\
\hline & 55 & 23 & 4 & 0 & 58 & 20 & 18 & 69 \\
\hline & 82 & 10 & 3 & 0 & 73 & 11 & 11 & 83 \\
\hline T19 & 0 & 0 & 100 & 0 & 0 & 99 & 0 & 0 \\
\hline & 0 & 3 & 97 & 0 & 0 & 99 & 0 & 1 \\
\hline P1 & 18 & 28 & 48 & 4 & 46 & 33 & 6 & 30 \\
\hline & 32 & 26 & 36 & 1 & 50 & 37 & 1 & 38 \\
\hline & 20 & 68 & 5 & 0 & 24 & 71 & 2 & 31 \\
\hline
\end{tabular}




\section{Assessment of Ecological Conditions and Potential Effects of Water, Wyoming and Montana, 2005-08}

Table 6. Macroinvertebrate community metrics for streams in the Powder River structural basin, Wyoming and Montana, 2005-08. -Continued

[Shaded cells indicate main-stem sampling sites on the Tongue or Powder River. \%, percent]

\begin{tabular}{|c|c|c|c|c|c|c|}
\hline \multirow[b]{2}{*}{$\begin{array}{c}\text { Site } \\
\text { number } \\
\text { (fig. 1) }\end{array}$} & \multirow[b]{2}{*}{ Abbreviated site name } & \multirow[b]{2}{*}{$\begin{array}{c}\text { Sample } \\
\text { date }\end{array}$} & \multicolumn{4}{|c|}{ Taxa richness } \\
\hline & & & Total & $\begin{array}{c}\text { Ephemeroptera } \\
\text { richness }\end{array}$ & $\begin{array}{c}\text { Trichoptera } \\
\text { richness }\end{array}$ & $\begin{array}{l}\text { Diptera } \\
\text { richness }\end{array}$ \\
\hline \multirow[t]{3}{*}{ P2 } & Powder River below Salt Creek & $7 / 21 / 2005$ & 20 & 4 & 3 & 12 \\
\hline & & $7 / 25 / 2006$ & 33 & 4 & 3 & 21 \\
\hline & & $8 / 30 / 2007$ & 35 & 6 & 5 & 18 \\
\hline \multirow{2}{*}{ P3 } & & $8 / 24 / 2007$ & 21 & 3 & 3 & 12 \\
\hline & & $7 / 21 / 2008$ & 9 & 1 & 2 & 4 \\
\hline \multirow[t]{4}{*}{ P4 } & Powder River below Burger Draw & $7 / 22 / 2005$ & 17 & 4 & 2 & 9 \\
\hline & & $7 / 27 / 2006$ & 24 & 1 & 4 & 14 \\
\hline & & $8 / 22 / 2007$ & 10 & 3 & 2 & 3 \\
\hline & & $7 / 23 / 2008$ & 10 & 1 & 2 & 6 \\
\hline P6 & & $8 / 20 / 2007$ & 25 & 4 & 5 & 5 \\
\hline \multirow[t]{3}{*}{ P7 } & Crazy Woman Creek near mouth & $7 / 12 / 2005$ & 17 & 6 & 3 & 4 \\
\hline & & $8 / 21 / 2007$ & 28 & 5 & 4 & 12 \\
\hline & & $8 / 20 / 2008$ & 26 & 7 & 3 & 12 \\
\hline \multirow[t]{3}{*}{ P8 } & Powder River below Crazy Woman Creek & $7 / 23 / 2005$ & 34 & 4 & 2 & 24 \\
\hline & & $8 / 21 / 2007$ & 17 & 4 & 2 & 6 \\
\hline & & $7 / 25 / 2008$ & 16 & 7 & 1 & 5 \\
\hline \multirow[t]{3}{*}{ P9 } & Powder River above Clear Creek & $7 / 24 / 2005$ & 32 & 6 & 1 & 21 \\
\hline & & $7 / 26 / 2007$ & 34 & 10 & 4 & 12 \\
\hline & & $7 / 29 / 2008$ & 23 & 9 & 2 & 7 \\
\hline \multirow{2}{*}{$\mathrm{P} 10$} & Clear Creek & $9 / 13 / 2005$ & 17 & 6 & 4 & 3 \\
\hline & & $8 / 21 / 2006$ & 34 & 5 & 6 & 12 \\
\hline P13 & Powder River at Broadus & $7 / 19 / 2005$ & 10 & 5 & 1 & 3 \\
\hline & & $8 / 2 / 2006$ & 28 & 4 & 6 & 12 \\
\hline & & $7 / 25 / 2007$ & 18 & 7 & 3 & 4 \\
\hline & & $7 / 31 / 2008$ & 12 & 6 & 2 & 2 \\
\hline P14 & Little Powder River at Highway 59 & $8 / 8 / 2007$ & 29 & 1 & 2 & 10 \\
\hline P15 & Little Powder River above Dry Creek & $6 / 13 / 2005$ & 12 & 2 & 2 & 6 \\
\hline & & $6 / 23 / 2006$ & 29 & 2 & 4 & 15 \\
\hline & & $8 / 7 / 2007$ & 19 & 4 & 3 & 8 \\
\hline & & $8 / 18 / 2008$ & 21 & 3 & 2 & 8 \\
\hline P16 & Little Powder River at Biddle & $6 / 27 / 2005$ & 37 & 2 & 3 & 22 \\
\hline & & $8 / 9 / 2007$ & 26 & 4 & 3 & 11 \\
\hline P17 & Powder River below Little Powder River & $7 / 21 / 2005$ & 20 & 6 & 3 & 8 \\
\hline & & $7 / 25 / 2007$ & 26 & 9 & 3 & 8 \\
\hline P18 & Powder River near Locate & $7 / 22 / 2005$ & 9 & 4 & 2 & 2 \\
\hline & & $7 / 24 / 2007$ & 14 & 4 & 3 & 3 \\
\hline $\mathrm{C} 3$ & Cheyenne River near Dull Center & $6 / 27 / 2005$ & 28 & 2 & 2 & 18 \\
\hline $\mathrm{C} 4$ & Little Thunder Creek & $6 / 9 / 2005$ & 16 & 1 & 0 & 11 \\
\hline $\mathrm{C} 6$ & Cheyenne River near Spencer & $6 / 6 / 2005$ & 18 & 1 & 1 & 12 \\
\hline & & $6 / 15 / 2007$ & 7 & 0 & 0 & 7 \\
\hline B1 & Belle Fourche River & $6 / 29 / 2005$ & 17 & 1 & 1 & 8 \\
\hline
\end{tabular}


Table 6. Macroinvertebrate community metrics for streams in the Powder River structural basin, Wyoming and Montana, 2005-08. - Continued

[Shaded cells indicate main-stem sampling sites on the Tongue or Powder River. \%, percent]

\begin{tabular}{|c|c|c|c|c|c|c|c|c|}
\hline \multirow[b]{2}{*}{$\begin{array}{c}\text { Site } \\
\text { number } \\
\text { (fig. 1) }\end{array}$} & \multicolumn{4}{|c|}{ Taxa relative abundance } & \multicolumn{3}{|c|}{ Functional feeding mode relative abundance } & \multirow{2}{*}{$\begin{array}{c}\text { Tolerance } \\
\text { Intolerant } \\
\text { macro- } \\
\text { invertebrates } \\
(\%)\end{array}$} \\
\hline & $\begin{array}{c}\text { Ephemeroptera } \\
\text { abundance } \\
(\%)\end{array}$ & $\begin{array}{c}\text { Trichoptera } \\
\text { abundance } \\
(\%)\end{array}$ & $\begin{array}{c}\text { Diptera } \\
\text { abundance } \\
(\%)\end{array}$ & $\begin{array}{c}\text { Noninsect } \\
\text { abundance } \\
(\%)\end{array}$ & $\begin{array}{c}\text { Collector- } \\
\text { gatherer } \\
\text { abundance } \\
(\%)\end{array}$ & $\begin{array}{c}\text { Filterer- } \\
\text { collector } \\
\text { abundance } \\
(\%)\end{array}$ & $\begin{array}{c}\text { Scraper } \\
\text { abundance } \\
(\%)\end{array}$ & \\
\hline \multirow[t]{3}{*}{ P2 } & 9 & 5 & 86 & 0 & 13 & 59 & 3 & 3 \\
\hline & 6 & 10 & 83 & 0 & 57 & 29 & 0 & 10 \\
\hline & 20 & 44 & 26 & 2 & 29 & 52 & 2 & 16 \\
\hline \multirow[t]{4}{*}{ P3 } & 16 & 10 & 74 & 0 & 12 & 76 & 7 & 16 \\
\hline & 14 & 57 & 26 & 1 & 18 & 73 & 1 & 19 \\
\hline & 5 & 59 & 35 & 0 & 8 & 90 & 0 & 5 \\
\hline & 0 & 10 & 89 & 0 & 0 & 98 & 0 & 2 \\
\hline \multirow[t]{4}{*}{ P4 } & 9 & 46 & 45 & 0 & 12 & 71 & 0 & 14 \\
\hline & 1 & 36 & 56 & 5 & 5 & 78 & 5 & 8 \\
\hline & 3 & 2 & 94 & 0 & 4 & 96 & 0 & 4 \\
\hline & 0 & 4 & 95 & 0 & 1 & 99 & 0 & 1 \\
\hline \multirow[t]{4}{*}{ P5 } & 12 & 18 & 69 & 1 & 13 & 85 & 0 & 15 \\
\hline & 8 & 59 & 20 & 0 & 17 & 64 & 1 & 31 \\
\hline & 6 & 50 & 42 & 1 & 9 & 84 & 1 & 7 \\
\hline & 1 & 2 & 97 & 0 & 1 & 99 & 0 & 1 \\
\hline \multirow{2}{*}{ P6 } & 53 & 23 & 15 & 5 & 59 & 32 & 4 & 31 \\
\hline & 14 & 32 & 2 & 1 & 53 & 31 & 10 & 49 \\
\hline \multirow[t]{3}{*}{ P7 } & 5 & 6 & 86 & 0 & 6 & 92 & 0 & 4 \\
\hline & 23 & 45 & 13 & 2 & 36 & 46 & 4 & 30 \\
\hline & 37 & 37 & 22 & 0 & 37 & 45 & 3 & 36 \\
\hline \multirow[t]{3}{*}{ P8 } & 6 & 20 & 70 & 3 & 24 & 51 & 0 & 9 \\
\hline & 2 & 9 & 88 & 1 & 2 & 95 & 0 & 2 \\
\hline & 4 & 2 & 89 & 1 & 4 & 90 & 1 & 5 \\
\hline \multirow[t]{3}{*}{ P9 } & 11 & 4 & 80 & 2 & 21 & 31 & 1 & 10 \\
\hline & 62 & 22 & 11 & 1 & 62 & 27 & 2 & 61 \\
\hline & 60 & 4 & 34 & 0 & 59 & 32 & 1 & 59 \\
\hline \multirow[t]{4}{*}{ P10 } & 74 & 20 & 0 & 0 & 79 & 20 & 1 & 94 \\
\hline & 18 & 42 & 9 & 4 & 45 & 42 & 9 & 74 \\
\hline & 60 & 10 & 14 & 4 & 69 & 12 & 5 & 73 \\
\hline & 43 & 21 & 7 & 1 & 57 & 21 & 14 & 63 \\
\hline \multirow[t]{2}{*}{ P11 } & 49 & 32 & 16 & 0 & 40 & 49 & 10 & 52 \\
\hline & 5 & 67 & 11 & 3 & 23 & 69 & 3 & 37 \\
\hline \multirow[t]{2}{*}{ P11 } & 59 & 28 & 5 & 2 & 64 & 27 & 4 & 57 \\
\hline & 53 & 23 & 16 & 1 & 52 & 39 & 8 & 66 \\
\hline P12 & 34 & 32 & 29 & 3 & 37 & 49 & 3 & 30 \\
\hline & 69 & 5 & 23 & 0 & 69 & 23 & 3 & 66 \\
\hline & 38 & 5 & 55 & 1 & 38 & 57 & 1 & 41 \\
\hline P13 & 66 & 1 & 33 & 0 & 66 & 34 & 0 & 66 \\
\hline & 15 & 55 & 27 & 2 & 24 & 54 & 6 & 19 \\
\hline & 51 & 26 & 20 & 0 & 54 & 42 & 1 & 55 \\
\hline & 56 & 4 & 39 & 0 & 55 & 44 & 0 & 55 \\
\hline P14 & 6 & 8 & 7 & 58 & 64 & 12 & 10 & 18 \\
\hline P15 & 1 & 2 & 95 & 0 & 2 & 96 & 0 & 1 \\
\hline & 1 & 91 & 4 & 1 & 3 & 91 & 1 & 15 \\
\hline & 2 & 88 & 8 & 1 & 3 & 91 & 0 & 7 \\
\hline & 8 & 73 & 13 & 0 & 10 & 79 & 1 & 10 \\
\hline P16 & 3 & 47 & 36 & 2 & 13 & 58 & 8 & 7 \\
\hline & 38 & 23 & 26 & 3 & 39 & 28 & 7 & 34 \\
\hline P17 & 59 & 18 & 21 & 0 & 69 & 26 & 1 & 61 \\
\hline & 20 & 36 & 40 & 1 & 23 & 65 & 2 & 22 \\
\hline P18 & 74 & 7 & 19 & 0 & 74 & 25 & 0 & 74 \\
\hline & 21 & 73 & 3 & 0 & 19 & 77 & 0 & 26 \\
\hline C3 & 3 & 0 & 44 & 51 & 35 & 18 & 42 & 0 \\
\hline $\mathrm{C} 4$ & 1 & 0 & 98 & 1 & 3 & 95 & 0 & 0 \\
\hline C6 & 0 & 0 & 98 & 1 & 2 & 94 & 1 & 0 \\
\hline & 0 & 0 & 100 & 0 & 8 & 48 & 0 & 0 \\
\hline B1 & 0 & 1 & 78 & 19 & 20 & 77 & 0 & 1 \\
\hline
\end{tabular}




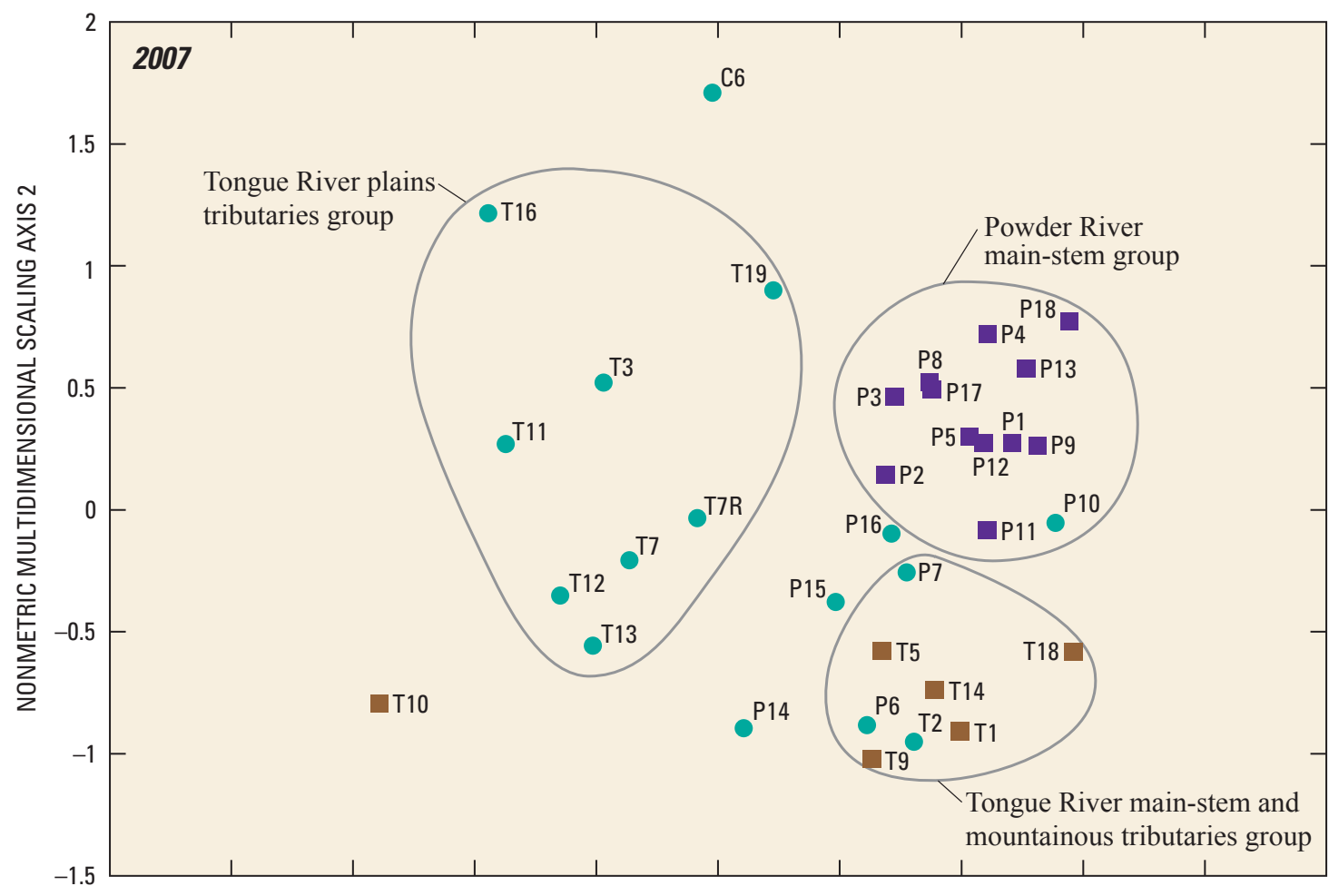

\section{EXPLANATION}

Stream

- Tributary

- Powder River main stem

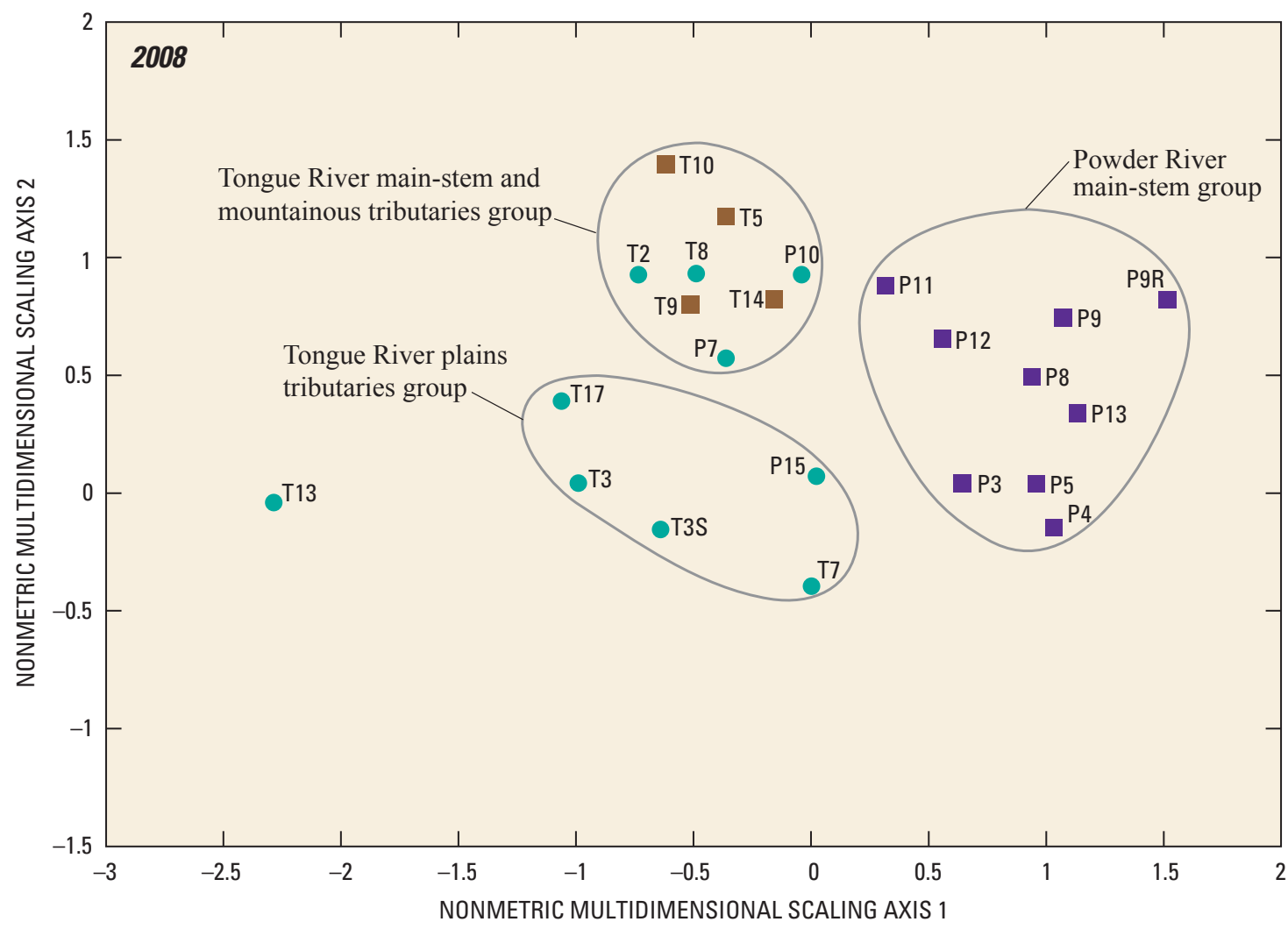

Figure 5. Similarities of macroinvertebrate community taxonomic composition within stream groups depicted by nonmetric multidimensional scaling ordinations, Powder River structural basin, Wyoming and Montana, 2007-08.
- Tongue River main stem

R Replicate

S Split 

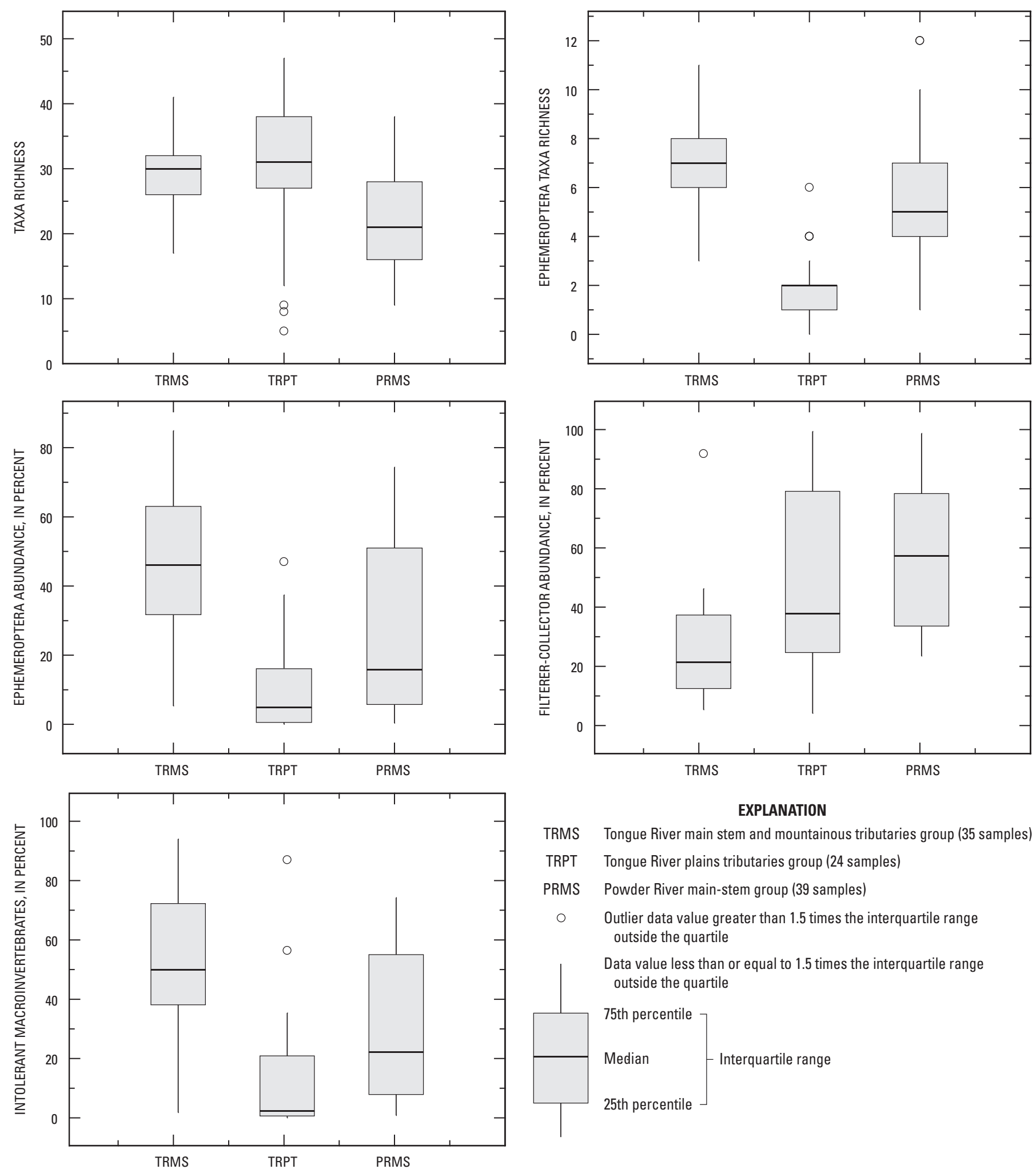

\section{EXPLANATION}

TRMS Tongue River main stem and mountainous tributaries group (35 samples)

TRPT Tongue River plains tributaries group (24 samples)

PRMS Powder River main-stem group (39 samples)

$\circ \quad$ Outlier data value greater than 1.5 times the interquartile range outside the quartile

Data value less than or equal to 1.5 times the interquartile range outside the quartile

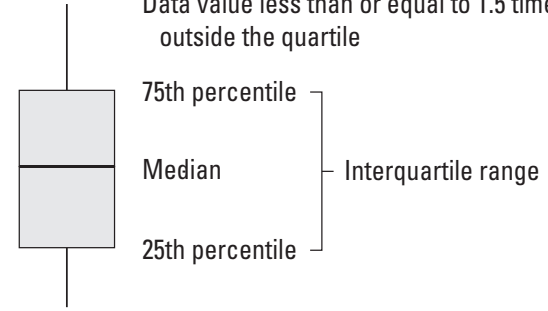

Figure 6. Selected macroinvertebrate community metrics by stream group, Powder River structural basin, Wyoming and Montana, 2005-08. 
Table 7. Analysis of variance in macroinvertebrate community metrics, main-stem Tongue River and main-stem Powder River, Wyoming and Montana, 2005-08.

[Significance tested by Tukey method at probability level less than 0.05]

\begin{tabular}{|c|c|c|c|c|}
\hline Metric & \multicolumn{2}{|c|}{$\begin{array}{c}\text { Main-stem Tongue River } \\
\text { (sites T1, T5, T9, T10, T14, and T18) }\end{array}$} & \multicolumn{2}{|c|}{$\begin{array}{c}\text { Main-stem Powder River } \\
\text { (sites P1-P5, P8, P9, P11-P13, P17, P18) }\end{array}$} \\
\hline Ephemeroptera taxa richness & None & None & $\begin{array}{l}\mathrm{P} 4 \text { to } \mathrm{P} 9, \mathrm{P} 4 \text { to } \mathrm{P} 11, \\
\mathrm{P} 4 \text { to } \mathrm{P} 12\end{array}$ & None \\
\hline Trichoptera taxa richness & None & None & None & $\begin{array}{l}2005 \text { to } 2006, \\
2005 \text { to } 2007, \\
2006 \text { to } 2008, \\
2007 \text { to } 2008\end{array}$ \\
\hline Diptera taxa richness & None & None & None & $\begin{array}{l}2005 \text { to } 2008, \\
2006 \text { to } 2007, \\
2006 \text { to } 2008\end{array}$ \\
\hline Ephemeroptera relative abundance & None & None & $\begin{array}{l}\mathrm{P} 4 \text { to } \mathrm{P} 9, \mathrm{P} 4 \text { to } \mathrm{P} 12, \\
\mathrm{P} 4 \text { to } \mathrm{P} 13, \mathrm{P} 4 \text { to } \mathrm{P} 18 \text {, } \\
\mathrm{P} 8 \text { to } \mathrm{P} 12, \mathrm{P} 8 \text { to } \mathrm{P} 13\end{array}$ & None \\
\hline Diptera relative abundance & None & None & $\begin{array}{c}\mathrm{P} 4 \text { to } \mathrm{P} 11, \mathrm{P} 8 \text { to } \mathrm{P} 11, \\
\mathrm{P} 8 \text { to } \mathrm{P} 18\end{array}$ & None \\
\hline Noninsect relative abundance & $\begin{array}{c}\text { T10 to } \mathrm{T} 9, \mathrm{~T} 10 \text { to } \mathrm{T} 14, \\
\mathrm{~T} 10 \text { to } \mathrm{T} 18\end{array}$ & None & None & None \\
\hline Dominant taxa percent & None & None & None & None \\
\hline Collector-gatherer relative abundance & None & None & $\begin{array}{l}\mathrm{P} 3 \text { to } \mathrm{P} 13, \mathrm{P} 4 \text { to } \mathrm{P} 9 \\
\mathrm{P} 4 \text { to } \mathrm{P} 11, \mathrm{P} 4 \text { to } \mathrm{P} 12, \\
\mathrm{P} 4 \text { to } \mathrm{P} 13, \mathrm{P} 5 \text { to } \mathrm{P} 13\end{array}$ & None \\
\hline Filterer-collector relative abundance & None & None & $\begin{array}{l}\mathrm{P} 3 \text { to } \mathrm{P} 9, \mathrm{P} 4 \text { to } \mathrm{P} 9 \\
\text { P5 to } \mathrm{P} 9\end{array}$ & None \\
\hline Scraper relative abundance & None & 2006 to 2008 & None & None \\
\hline Intolerant relative abundance & None & None & $\begin{array}{l}\mathrm{P} 3 \text { to } \mathrm{P} 11, \mathrm{P} 4 \text { to } \mathrm{P} 11, \\
\mathrm{P} 4 \text { to } \mathrm{P} 12, \mathrm{P} 4 \text { to } \mathrm{P} 13, \\
\mathrm{P} 8 \text { to } \mathrm{P} 11, \mathrm{P} 8 \text { to } \mathrm{P} 13\end{array}$ & None \\
\hline
\end{tabular}

downstream (sites P9, P11-P13, P17, and P18). Metric values for Ephemeroptera taxa richness, Ephemeroptera relative abundance, and intolerant macroinvertebrate relative abundance tended to be lower, whereas Diptera relative abundance and filterer-collector relative abundance tended to be higher, at sites P3-P5 and P8 than at other sites on the main-stem Powder River (fig. 8). The affected metrics included measures of taxa richness, taxa relative abundance, feeding modes, and tolerance, which were indicative of communitywide differences.

Taxa richness, Trichoptera taxa richness, Diptera taxa richness, and relative abundance of Trichoptera varied significantly ( $\mathrm{p}<0.05$ ) by year during $2005-08$ (table 7). As shown in figure 8, using Diptera taxa richness as an example, the metric values in 2006 were higher than in 2005 and 2007-08. Taxa richness and the Trichoptera metrics also were higher in 2006 than the other years. As noted for the main-stem Tongue River, the extended drought and severe low-flow conditions in 2006 might be at least partly the cause of the year-to-year variation in metric values from the main-stem Powder River.

\section{Tributaries}

Macroinvertebrate communities at sites on tributaries sometimes showed variation between 2006 and other years $(2005,2007-08)$ that resembled the patterns observed on the Tongue River and Powder River. For example, metrics with higher values in 2006 than other years included Diptera taxa richness and scraper abundance in Goose Creek (site T2), taxa richness and Trichoptera abundance in Clear Creek (site P10), and taxa richness and Diptera richness in the Little Powder River (site P15; table 6). Comparison of metrics from upper Youngs Creek (site T3) to lower Youngs Creek (site T4) indicated lower relative abundance of Ephemeroptera and higher relative abundance of noninsects at the lower site compared to the upper site, but these data should be used with caution because only 2 years (2005-06) of data were available from site $\mathrm{T} 4$ and may reflect natural spatial variation with lower elevation and increased drainage area. Metrics for the lower site on Squirrel Creek (site T7) indicated relatively low taxa richness in 2008 compared to 2005 and 2007. The 

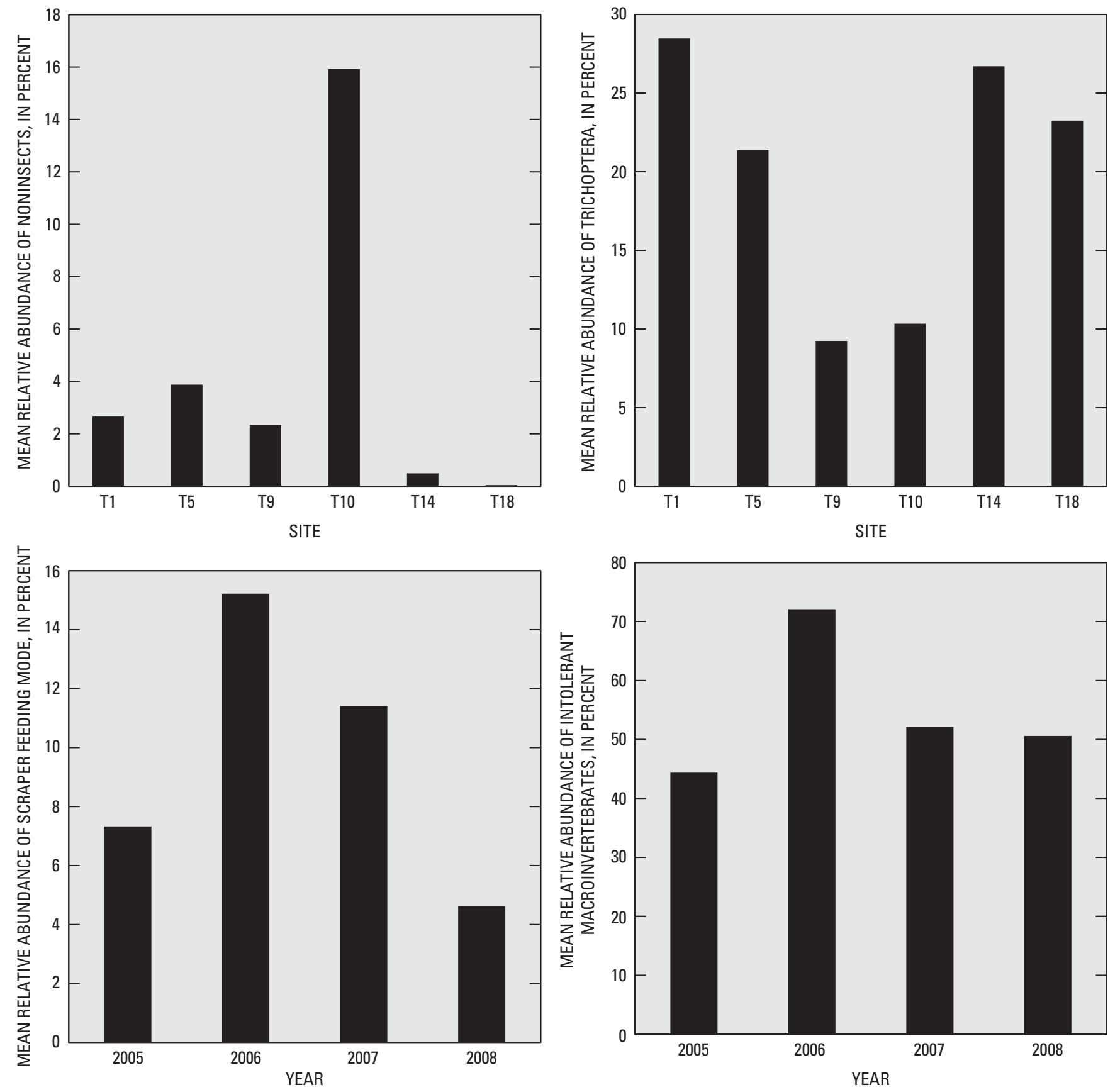

Figure 7. Mean values for selected macroinvertebrate community metrics by site and by year, main-stem Tongue River, Wyoming and Montana, 2005-08.

macroinvertebrate communities at site $\mathrm{T} 7$ might be affected by intermittent streamflow. The upper site on Squirrel Creek (site T6) did not have sufficient riffle habitat for sampling; therefore, no metric data are available for comparison with the lower site. Interpretation of data from other tributaries, such as Crazy Woman Creek, Hanging Woman Creek, and Otter Creek is limited by the small number of samples available and variability in community composition, which in turn might be related to intermittent streamflow at some of the sites and natural spatial variation.

\section{Effects of Environmental Variables on Macroinvertebrate Communities}

Analysis of environmental variables during 2005-08 (table 3) indicated grouping of sites, similar to the TRMS, TRPT, and PRMS stream groups observed for the macroinvertebrate communities. The PCA ordination of sites and environmental variables from 2007 is shown in figure 9 as an example. Sites on the main-stem Tongue River tended to group along the right side of the ordination (positive correlation with principal components 

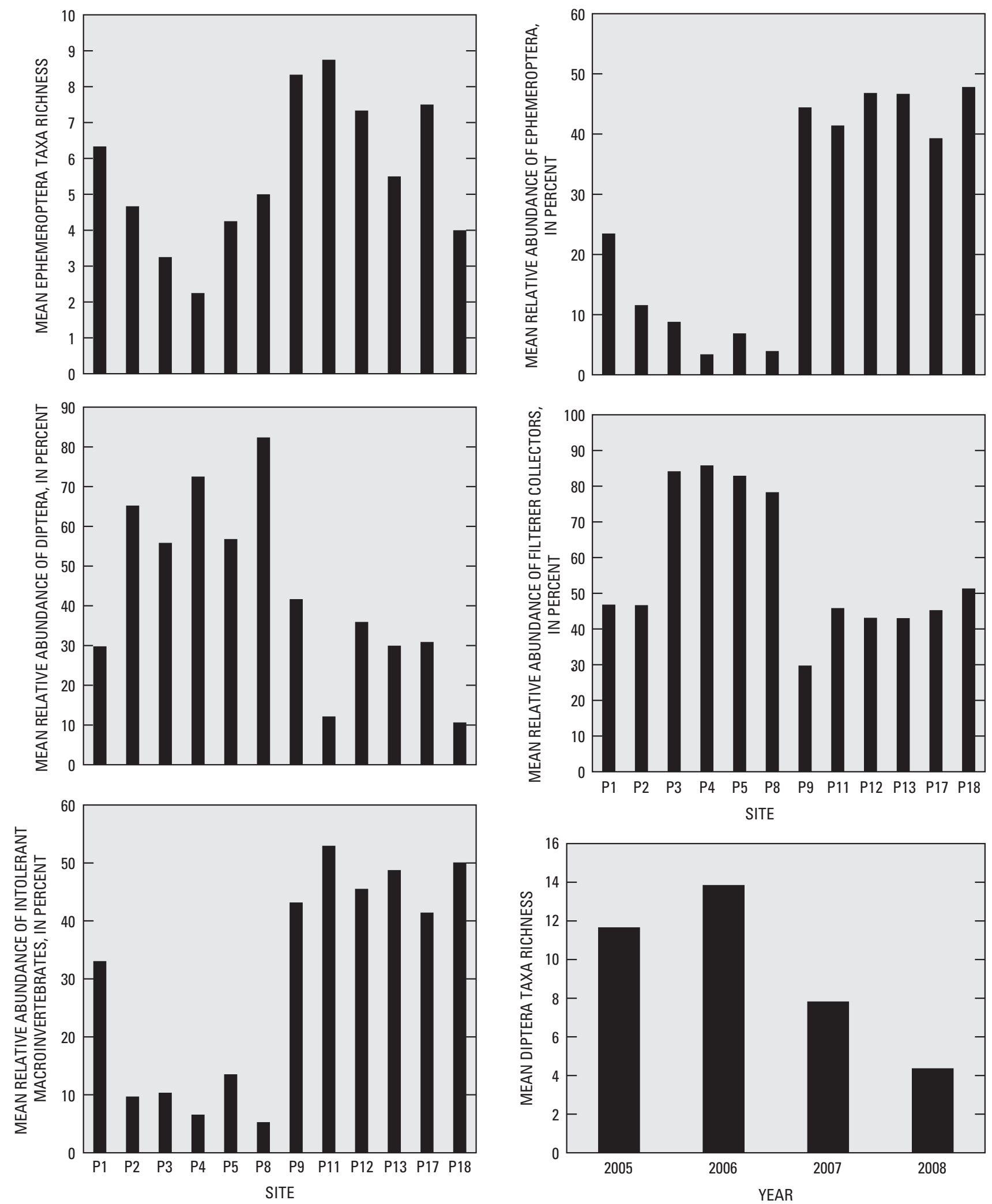

Figure 8. Mean values for selected macroinvertebrate metrics by site and by year, main-stem Powder River, Wyoming and Montana, 2005-08. 


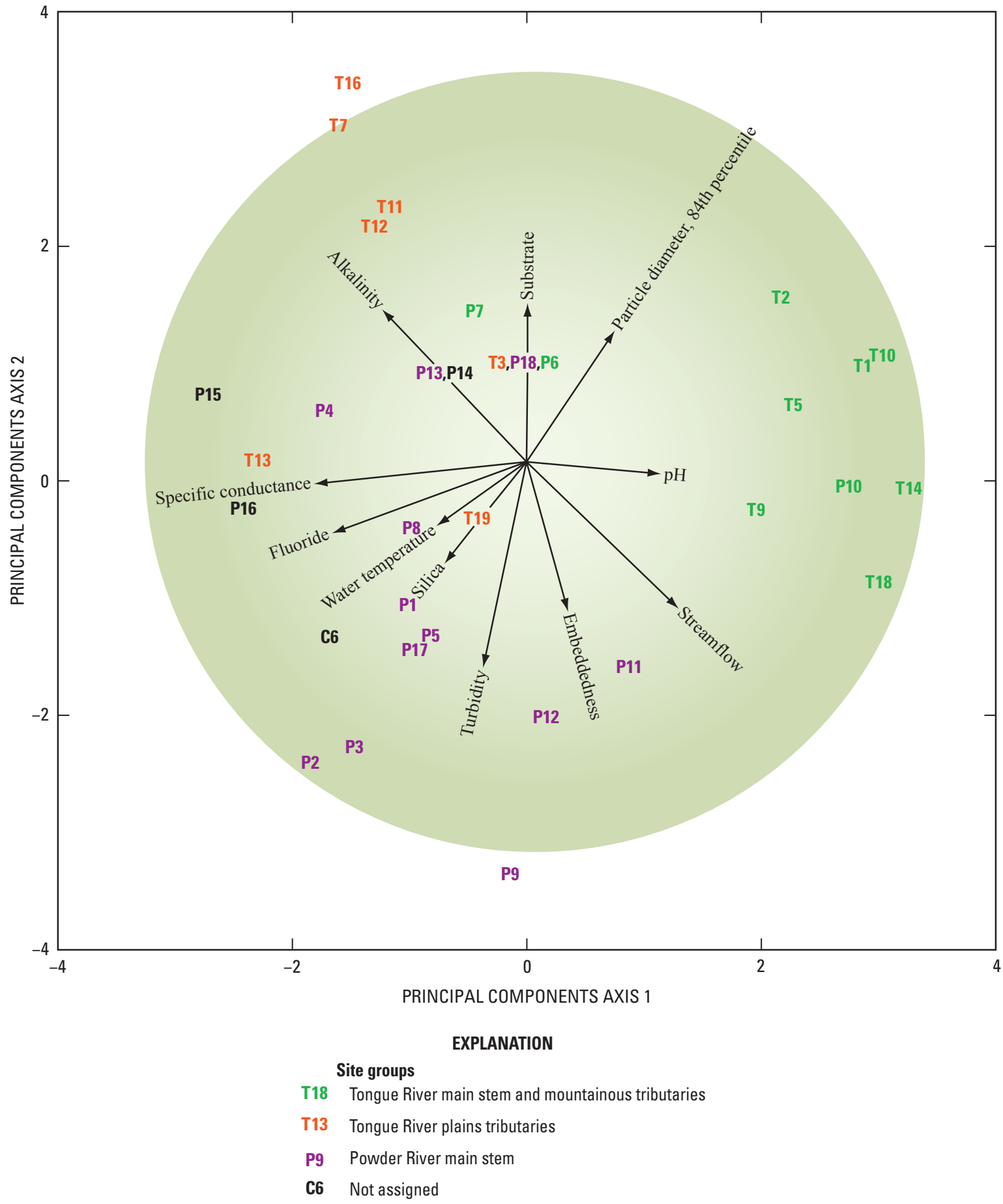

Figure 9. Principal components ordination showing relations among sampling sites based on selected environmental variables, Powder River structural basin, Wyoming and Montana, 2007. 
Table 8. Environmental variables best correlated with macroinvertebrate community taxonomic composition, by stream group and year, Powder River structural basin, Wyoming and Montana, 2005-08.

$\left[\mathrm{D}_{84}\right.$, diameter of the 84 th percentile of particles $]$

\begin{tabular}{|c|c|c|}
\hline Year & $\begin{array}{l}\text { Spearman } \\
\text { correlation } \\
\text { coefficient }\end{array}$ & Environmental variables \\
\hline \multicolumn{3}{|c|}{ Main-stem Tongue River } \\
\hline 2005 & 0.52 & $\begin{array}{l}\text { Streamflow, specific conductance, } \\
\text { dissolved oxygen }\end{array}$ \\
\hline 2006 & 0.21 & Streamflow \\
\hline 2007 & 0.89 & $\begin{array}{l}\text { Specific conductance, dissolved oxygen, } \\
\text { pH, turbidity }\end{array}$ \\
\hline 2008 & 0.77 & $\begin{array}{l}\text { Streamflow, specific conductance, dissolved } \\
\text { oxygen, } \mathrm{pH} \text {, and water temperature }\end{array}$ \\
\hline \multicolumn{3}{|c|}{ Tongue River plains tributaries } \\
\hline 2005 & 0.47 & Streamflow and specific conductance \\
\hline 2006 & 0.54 & $\begin{array}{l}\text { Specific conductance, dissolved oxygen, } \\
\text { and substrate }\end{array}$ \\
\hline 2007 & 0.61 & Streamflow and specific conductance \\
\hline 2008 & 0.66 & $\begin{array}{l}\text { Dissolved oxygen, embeddedness, and } \\
\text { water temperature }\end{array}$ \\
\hline \multicolumn{3}{|r|}{ Main-stem Powder River } \\
\hline 2005 & 0.38 & alkalinity and $\mathrm{pH}$ \\
\hline 2006 & 0.70 & $\begin{array}{l}\text { turbidity, embeddedness, and } \\
\text { water temperature }\end{array}$ \\
\hline 2007 & 0.56 & $\begin{array}{l}\text { turbidity, embeddedness, } \mathrm{D}_{84} \text {, specific } \\
\text { conductance, and fluoride }\end{array}$ \\
\hline 2008 & 0.50 & $\begin{array}{l}\text { turbidity, embeddedness, } \mathrm{D}_{84} \text {, alkalinity, } \\
\text { and streamflow }\end{array}$ \\
\hline
\end{tabular}

(PC) axis 1; fig. 9), whereas sites on plains tributaries to the Tongue River tended to have positive correlation with PC axis 2 (upper left), and sites on the main-stem Powder River tended to have negative correlation with PC axis 2 (lower left). The 2007 eigenvectors indicated PC axis 1 was correlated with chemical variables such as specific conductance and fluoride; $\mathrm{PC}$ axis 2 was correlated with physical variables such as turbidity and embeddedness. PCA ordinations for 2005-06 and 2008 showed similar results to those for 2007, due to similarities in values of environmental variables within the stream groups.

Given the differences in environmental variables between stream groups and differences in the number of sites sampled per year, PCA ordinations were repeated separately by stream group (TRMS, TRPT, and PRMS) and by year. Collinear variables were removed on a stepwise basis for each stream group and year, but in general, the pebble count measure, $\mathrm{D}_{84}$, was used as an indicator of the $\mathrm{D}_{50}$ and percent substrate less than $2 \mathrm{~mm}$; instantaneous streamflow was used as an indicator of microhabitat depth and velocity, and specific conductance was used as an indicator of major ions including sodium, potassium, chloride, sulfate, and total dissolved solids. Calcium, magnesium, and alkalinity were often but not always correlated with specific conductance. The selected environmental variables that produced the best correlation with the macroinvertebrate community taxonomy data using the BEST routine (Clarke and Gorley, 2006) are listed in table 8.

\section{Main-Stem Tongue River}

The macroinvertebrate communities at sites along the main-stem Tongue River tended to be best correlated with streamflow, specific conductance, and dissolved-oxygen concentrations during 2005-08. Spearman correlation coefficients between the macroinvertebrate taxonomy data and environmental variables identified through PCA ranged from 0.21 in 2006 to 0.89 in 2007 (table 8). The correlation with streamflow might be attributed to the effects of consistently higher streamflow downstream from Tongue River Reservoir (sites T10, T14, and T18) than upstream from the reservoir (sites T1, T5, and T9; fig. 3A). Low streamflow during the drought year of 2006 (fig. 3C) might be a factor in the relatively low correlation coefficient of 0.21 for that year. Correlation of macroinvertebrate communities with specific conductance and dissolved-oxygen concentrations might also reflect the effects of Tongue River Reservoir. Specificconductance values at sites upstream from the reservoir increased from site $\mathrm{T} 1$ to $\mathrm{T} 9$ and tended to be higher upstream from the reservoir than downstream (fig. 4). Dissolved-oxygen concentrations tended to be lower downstream from the reservoir than upstream (fig. 10), although it should be noted that diel changes in dissolved-oxygen concentration were not measured.

\section{Tongue River Plains Tributaries}

Among all years, macroinvertebrate communities at sampling sites on plains tributaries to the Tongue River generally were best correlated with streamflow, specific conductance, and dissolved-oxygen concentrations (table 8). In 2005 for example, Hanging Woman Creek (sites T11-T13) had lower streamflow than other plains tributary sites such as Youngs Creek (sites T3 and T4), Squirrel Creek (site T7), Otter Creek (site T17), and Pumpkin Creek (site T19) and had higher specific-conductance values than most other plains tributary sites (fig. 10) perhaps due to higher intermittency in this system. The pattern of lower streamflow and higher specific conductance in Hanging Woman Creek than other plains tributaries also was repeated in 2007. Embeddedness, which was correlated with macroinvertebrate communities during 2008, ranged from less than 10 percent in Squirrel Creek (site T7) to 70 percent in Prairie Dog Creek (site T8) during 2008 (table 3).

\section{Main-Stem Powder River}

Macroinvertebrate communities at sites along the mainstem Powder River were correlated with turbidity and embeddedness in 2006-08, as well as other variables including $\mathrm{D}_{84}$, alkalinity, specific conductance, and streamflow in one or 

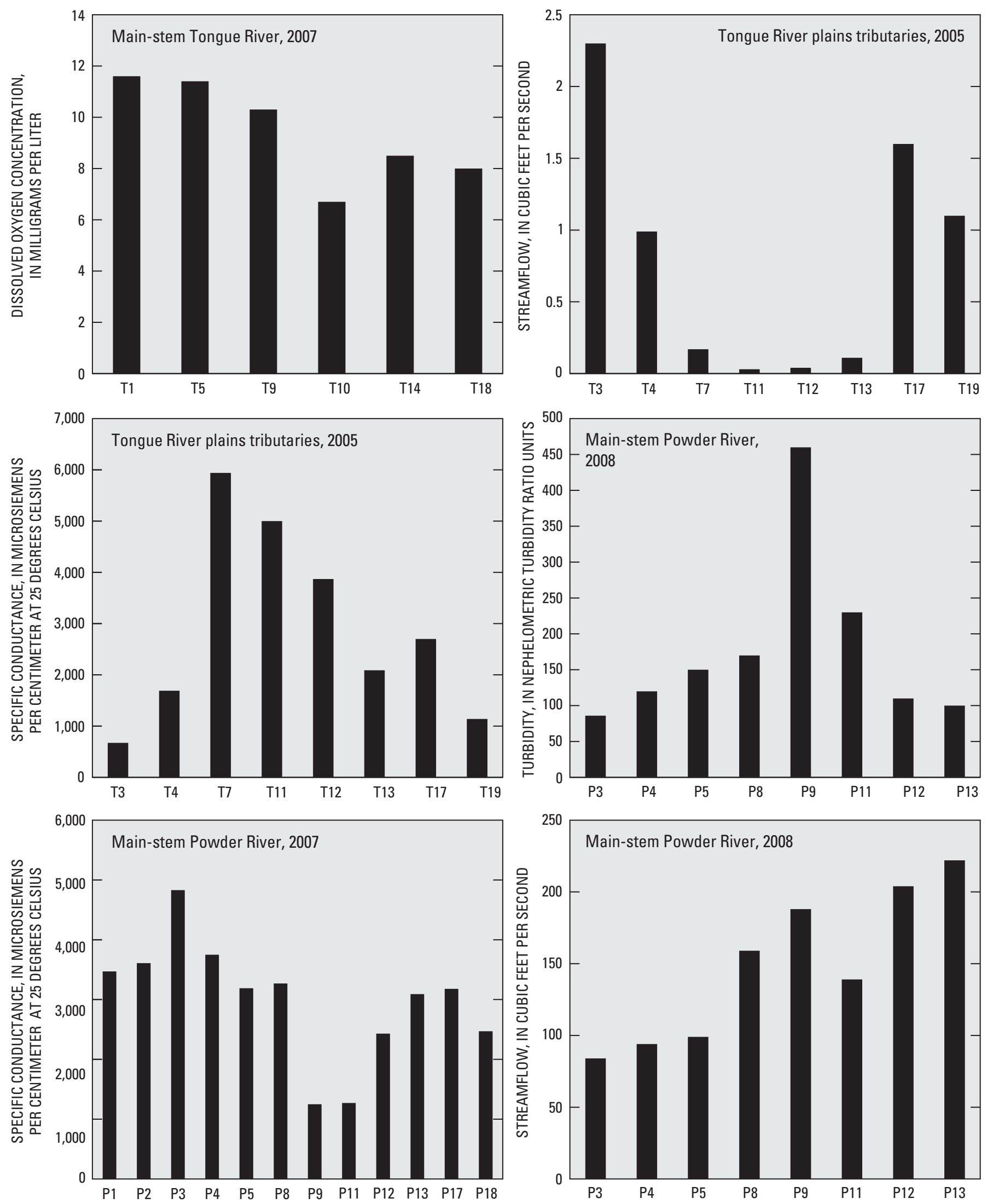

Figure 10. Selected environmental variables correlated with macroinvertebrate communities, Powder River structural basin, 2005-08. 
more years during 2005-08 (table 8). Embeddedness (table 3) and turbidity (fig. 10) varied inconsistently with downstream direction along the main-stem Powder River. Turbidity values were correlated with streamflow in $2005(\mathrm{r}=0.71)$ and 2007 $(\mathrm{r}=0.82)$ but not in 2006 or 2008 . Alkalinity concentrations, which were correlated with the macroinvertebrate communities in 2005 and 2008, were consistently higher in the Powder River below Burger Draw (site P4) than at other sites on the Powder River during 2005-08 (fig. 4; table 3). The Powder River at site $\mathrm{P} 4$ receives CBNG discharges from several drainages upstream. Specific conductance and dissolved-fluoride concentrations, which were correlated with the macroinvertebrate communities in 2007, reached maximum values in 2007 in the Powder River below Willow Creek (site P3; fig. 10 and table 3). Streamflow, which was correlated with macroinvertebrate communities in 2008, generally increased in the downstream direction along the main-stem Powder River in 2008 (fig. 10).

The BEST routine also was used to test if the environmental variables correlated with the macroinvertebrate communities of the middle reach of the Powder River (sites P3, P4, P5, and P8) were the same as those in the upper and lower reaches. The environmental data were renormalized, PCAs recalculated, and BEST correlation coefficients were recomputed using data from those four sites. The analyses confirmed that the environmental variables affecting the macroinvertebrate communities of the middle reach generally were the same as those affecting the upper and lower reaches.

As an additional test of the relations between macroinvertebrate communities and environmental variables in the main-stem Powder River, environmental variables from the PCA and BEST analyses were tested for correlation with the macroinvertebrate metrics (table 9). The maximum correlation coefficients occurred with streamflow and specific conductance. The relative abundance of Ephemeroptera increased with streamflow $(r=0.58)$ and decreased with specific conductance $(r=-0.56)$. The relative abundance of intolerant macroinvertebrates also decreased with increasing specific conductance $(\mathrm{r}=-0.58)$. The relative abundance of Trichoptera decreased with streamflow $(\mathrm{r}=-0.52)$.

The correlations observed between the environmental variables and the macroinvertebrate taxonomy and metrics indicate that variation in the macroinvertebrate communities can be partially explained by variation in the environmental variables that were measured. Other potential causes for variation in the macroinvertebrate communities include natural population dynamics, sampling techniques, environmental variables that were not measured, and fluctuations in the measured environmental variables beyond those observed at the time of sampling.

\section{Algal Communities}

Algal data are available from 2005 and 2007 for standing crop (http://waterdata.usgs.gov/wy/nwis/qw) and algae taxonomy (http://wy.water.usgs.gov/projects/atg/htms/data.htm). This section describes the algal data in terms of standing crop, community composition, and community traits that include autecological or environmental indicators. Algal samples were collected from riffles where available and from pools at sites where riffles were unavailable. The reader should be aware that differences in substrate (riffles compared to pools) can affect algal biovolume and abundance of specific taxa; however, algal relations with physical and chemical environment (autecology) are not significantly affected by differences in substrate (Potapova and Charles, 2005).

\section{Standing Crop}

Concentrations of chlorophyll- $a$ and ash-free dry mass (AFDM) in algae samples collected during 2005 and 2007 generally were small (table 10), indicating a relatively small amount of algal biomass in riffles. Median concentrations

Table 9. Correlation coefficients of macroinvertebrate community metrics with environmental variables, main-stem Powder River, Wyoming and Montana, 2005-08.

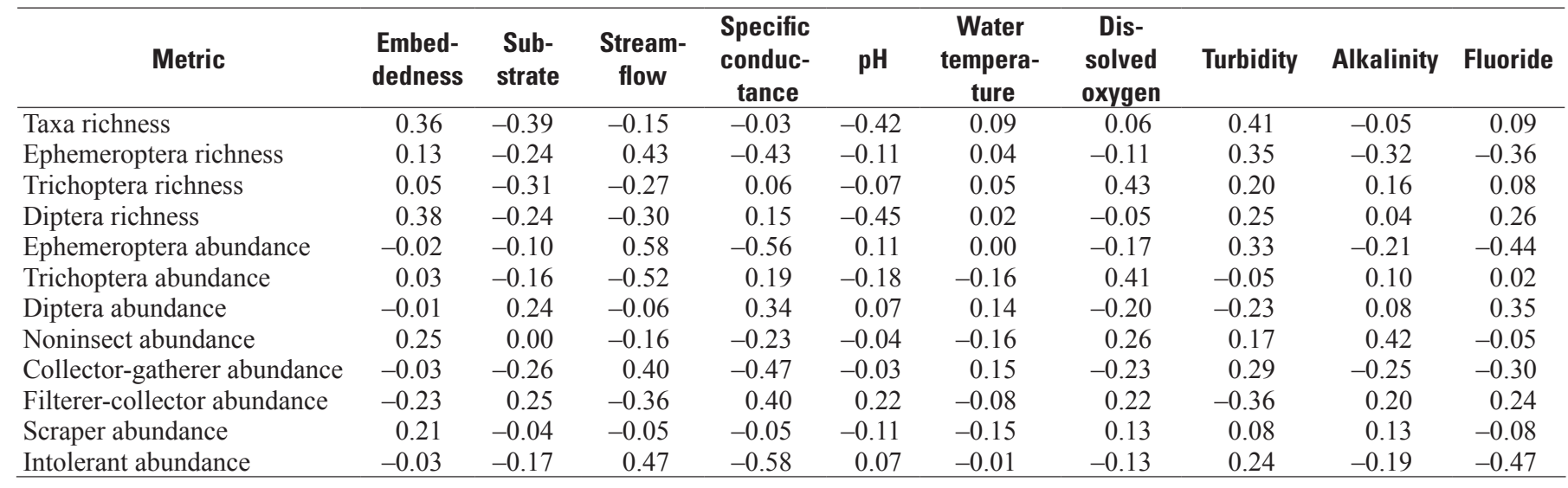


Table 10. Algal chlorophyll-a concentration, ash-free dry mass concentration, density, and biovolume, Powder River structural basin, Wyoming and Montana, 2005 and 2007.

[Shaded cells indicate main-stem sampling sites on the Tongue River or Powder River. mg, milligrams; $\mathrm{m}^{2}$, square meter; g, grams; $\mathrm{cm}^{3}$, cubic centimeters; NA, not analyzed or not available]

\begin{tabular}{|c|c|c|c|c|c|c|c|}
\hline $\begin{array}{c}\text { Site } \\
\text { number } \\
\text { (fig. 1) }\end{array}$ & Abbreviated site name & $\begin{array}{c}\text { Sample } \\
\text { date }\end{array}$ & Habitat & $\begin{array}{c}\text { Chlorophyll-a } \\
\left(\mathrm{mg} / \mathrm{m}^{2}\right)\end{array}$ & $\begin{array}{l}\text { Ash-free } \\
\text { dry mass } \\
\left(\mathrm{g} / \mathrm{m}^{2}\right)\end{array}$ & $\begin{array}{c}\text { Algal } \\
\text { density } \\
\text { (billion cells } / \mathrm{m}^{2} \text { ) }\end{array}$ & $\begin{array}{c}\text { Algal } \\
\text { biovolume } \\
\left(\mathrm{cm}^{3} / \mathrm{m}^{2}\right)\end{array}$ \\
\hline R1 & Upper Rosebud Creek & $7 / 9 / 2007$ & Pool & NA & NA & 3.29 & 2.46 \\
\hline \multirow[t]{2}{*}{$\mathrm{T} 1$} & Tongue River at Monarch & $8 / 15 / 2005$ & Riffle & 3.9 & 9.3 & NA & NA \\
\hline & & $8 / 29 / 2007$ & Riffle & 2.3 & 5.4 & 3.71 & 1.36 \\
\hline $\mathrm{T} 2$ & Goose Creek & $8 / 17 / 2005$ & Riffle & 2.1 & 5.2 & NA & NA \\
\hline $\mathrm{T} 4$ & Youngs Creek at mouth & $6 / 21 / 2007$ & Pool & NA & NA & 2.16 & 0.02 \\
\hline T5 & Tongue River below Youngs Creek & $8 / 29 / 2007$ & Riffle & 24.4 & 22.2 & 5.12 & 1.99 \\
\hline T6 & Upper Squirrel Creek & $7 / 11 / 2007$ & Pool & NA & NA & 4.34 & 0.13 \\
\hline $\mathrm{T} 7$ & Squirrel Creek at mouth & $7 / 12 / 2007$ & Riffle & 1.5 & 3.7 & 2.55 & 1.63 \\
\hline $\mathrm{T} 8$ & Prairie Dog Creek & $9 / 11 / 2007$ & Pool & NA & NA & 3.07 & 0.35 \\
\hline T9 & Tongue River at State line & $9 / 14 / 2005$ & Riffle & 47.1 & 49.4 & NA & NA \\
\hline $\mathrm{T} 13$ & Hanging Woman Creek at mouth & $7 / 10 / 2007$ & Riffle & 39.7 & 25.0 & 5.88 & 3.28 \\
\hline $\mathrm{T} 14$ & Tongue River at Birney Day School & $8 / 27 / 2007$ & Riffle & 1.8 & 5.7 & 3.88 & 2.58 \\
\hline $\mathrm{T} 15$ & Upper Otter Creek & $6 / 20 / 2007$ & Pool & NA & NA & 2.31 & 0.13 \\
\hline $\mathrm{T} 16$ & Middle Otter Creek & $6 / 20 / 2007$ & Riffle & 10.1 & 17.8 & 3.62 & 1.10 \\
\hline $\mathrm{T} 17$ & Otter Creek at mouth & $6 / 19 / 2007$ & Pool & NA & NA & 2.57 & 0.21 \\
\hline T18 & Tongue River below Brandenberg Bridge & $8 / 27 / 2007$ & Riffle & 5.5 & 6.2 & 3.43 & 0.73 \\
\hline T19 & Pumpkin Creek & $6 / 18 / 2007$ & Riffle & 0.6 & 3.8 & 2.49 & 0.06 \\
\hline \multirow[t]{2}{*}{ P1 } & Powder River above Salt Creek & $7 / 20 / 2005$ & Riffle & 10.1 & 12.4 & NA & NA \\
\hline & & $8 / 30 / 2007$ & Riffle & 18.3 & 14.1 & 4.04 & 0.13 \\
\hline \multirow[t]{2}{*}{$\mathrm{P} 2$} & Powder River below Salt Creek & $7 / 21 / 2005$ & Riffle & 18.1 & 23.3 & NA & NA \\
\hline & & $8 / 30 / 2007$ & Riffle & 16.6 & 13.1 & 4.11 & 0.11 \\
\hline P3 & Powder River below Willow Creek & $7 / 19 / 2005$ & Riffle & 2.9 & 17.4 & NA & NA \\
\hline P7 & & $8 / 21 / 2007$ & Riffle & 18.1 & 22.7 & 3.98 & 0.62 \\
\hline \multirow[t]{2}{*}{ P8 } & Powder River below Crazy Woman Creek & $7 / 23 / 2005$ & Riffle & 10.5 & 11.7 & NA & NA \\
\hline & & $8 / 21 / 2007$ & Riffle & 5.8 & 13.9 & 3.39 & 0.02 \\
\hline \multirow[t]{2}{*}{ P9 } & Powder River above Clear Creek & $7 / 24 / 2005$ & Riffle & 7.3 & 33.7 & NA & NA \\
\hline & & $7 / 26 / 2007$ & Riffle & 1.3 & 18.6 & 2.99 & 0.01 \\
\hline \multirow[t]{2}{*}{$\mathrm{P} 10$} & Clear Creek & $9 / 13 / 2005$ & Riffle & 12.3 & 21.8 & NA & NA \\
\hline & & $9 / 10 / 2007$ & Riffle & 15.2 & 18.0 & 3.96 & 0.67 \\
\hline \multirow[t]{2}{*}{ P11 } & Powder River below Clear Creek & $7 / 25 / 2005$ & Riffle & 54.1 & 22.0 & NA & NA \\
\hline & & $7 / 26 / 2007$ & Riffle & 8.4 & 25.1 & 3.32 & 0.0002 \\
\hline \multirow[t]{2}{*}{ P12 } & Powder River at Moorhead & $7 / 26 / 2005$ & Riffle & 71.3 & 61.6 & NA & NA \\
\hline & & $7 / 26 / 2007$ & Riffle & 4.0 & 32.2 & 3.87 & 0.02 \\
\hline P13 & Powder River at Broadus & $7 / 25 / 2007$ & Riffle & 0.5 & 25.1 & 4.23 & 0.43 \\
\hline $\mathrm{P} 14$ & Little Powder River at Highway 59 & $8 / 8 / 2007$ & Riffle & 443 & 87.7 & 3.55 & 0.51 \\
\hline \multirow[t]{2}{*}{$\mathrm{P} 15$} & Little Powder River above Dry Creek & $6 / 13 / 2005$ & Riffle & 2.7 & 13.0 & NA & NA \\
\hline & & $8 / 7 / 2007$ & Riffle & 5.5 & 11.6 & 3.22 & 0.16 \\
\hline P16 & Little Powder River at Biddle & $8 / 9 / 2007$ & Riffle & 4.4 & 4.1 & 2.47 & 0.02 \\
\hline P17 & Powder River below Little Powder River & $7 / 25 / 2007$ & Riffle & 0.2 & 326 & 3.70 & 0.63 \\
\hline P18 & Powder River near Locate & $7 / 24 / 2007$ & Riffle & 4.0 & 6.3 & 2.99 & 0.15 \\
\hline
\end{tabular}


Table 10. Algal chlorophyll- $a$ concentration, ash-free dry mass concentration, density, and biovolume, Powder River structural basin, Wyoming and Montana, 2005 and 2007.-Continued

[Shaded cells indicate main-stem sampling sites on the Tongue River or Powder River. mg, milligrams; $\mathrm{m}^{2}$, square meter; g, grams; $\mathrm{cm}^{3}$, cubic centimeters; NA, not analyzed or not available]

\begin{tabular}{|c|c|c|c|c|c|c|c|}
\hline $\begin{array}{c}\text { Site } \\
\text { number } \\
\text { (fig. 1) }\end{array}$ & Abbreviated site name & $\begin{array}{c}\text { Sample } \\
\text { date }\end{array}$ & Habitat & $\begin{array}{c}\text { Chlorophyll-a } \\
\left(\mathrm{mg} / \mathrm{m}^{2}\right)\end{array}$ & $\begin{array}{l}\text { Ash-free } \\
\text { dry mass } \\
\left(\mathrm{g} / \mathrm{m}^{2}\right)\end{array}$ & $\begin{array}{c}\text { Algal } \\
\text { density } \\
\text { (billion cells/m²) }\end{array}$ & $\begin{array}{c}\text { Algal } \\
\text { biovolume } \\
\left(\mathrm{cm}^{3} / \mathrm{m}^{2}\right)\end{array}$ \\
\hline $\mathrm{C} 1$ & Porcupine Creek & $6 / 12 / 2007$ & Pool & NA & $\mathrm{NA}$ & 2.08 & 0.36 \\
\hline \multirow[t]{2}{*}{ C3 } & Cheyenne River near Dull Center & $6 / 27 / 2005$ & Riffle & 4.7 & 16 & NA & NA \\
\hline & & $6 / 14 / 2007$ & Pool & NA & NA & 2.26 & 0.12 \\
\hline $\mathrm{C} 4$ & Little Thunder Creek & $6 / 22 / 2005$ & Riffle & 4.6 & 7.7 & NA & NA \\
\hline \multirow[t]{2}{*}{ C6 } & Cheyenne River near Spencer & $6 / 21 / 2005$ & Riffle & 0.6 & 6.1 & NA & NA \\
\hline & & $6 / 15 / 2007$ & Riffle & 4.0 & 11 & 2.99 & 0.45 \\
\hline \multirow[t]{2}{*}{ B1 } & Belle Fourche River & $6 / 29 / 2005$ & Riffle & 1.2 & 9.3 & NA & NA \\
\hline & & $6 / 12 / 2007$ & Pool & NA & NA & 0.65 & 0.05 \\
\hline $\mathrm{B} 2$ & Caballo Creek & $6 / 11 / 2007$ & Pool & NA & NA & 2.74 & 0.09 \\
\hline
\end{tabular}

of chlorophyll- $a$ were 4.6 milligrams per square meter $\left(\mathrm{mg} / \mathrm{m}^{2}\right)$ in 2005 and $6.2 \mathrm{mg} / \mathrm{m}^{2}$ in 2007. For comparison, all chlorophyll- $a$ concentrations in this study, with the exception of one, were less than the $100-150 \mathrm{mg} / \mathrm{m}^{2}$ concentration range associated with nuisance algal conditions (Stevenson and others, 1996). The exception was the chlorophyll- $a$ concentration of $443 \mathrm{mg} / \mathrm{m}^{2}$ from the Little Powder River at site P14 in 2007 (table 10) that might have been influenced by lessthan-optimal availability of riffle habitat for sampling. Median concentrations of AFDM were 14.5 grams per square meter $\left(\mathrm{g} / \mathrm{m}^{2}\right)$ in 2005 and 2007. AFDM concentrations were less than $100 \mathrm{~g} / \mathrm{m}^{2}$ in all samples with the exception of the 2007 sample from the Powder River below Little Powder River (site P17), which contained $326 \mathrm{~g} / \mathrm{m}^{2}$ (table 10) due to senescent algae or other organic matter in the sample. Concentrations of chlorophyll- $a$ and AFDM at ATG sites generally were similar to concentrations from the lower main-stem Yellowstone River and less than concentrations from the upper and middle mainstem Yellowstone River and tributaries (Peterson and Porter, 2002). Environmental variables affecting algal populations include nutrients, substrate, and light penetration (Stevenson and others, 1996).

Some of the measures of standing crop collected during 2007 (table 10) were moderately correlated with each other after removal of the outliers described previously. Chlorophyll- $a$ concentrations were significantly $(\mathrm{p}<0.05)$ correlated with AFDM $(r=0.50)$, algal density $(r=0.66)$, and algal biovolume $(\mathrm{r}=0.65)$. AFDM concentrations were significantly correlated with algal density $(r=0.62, p<0.05)$ but not biovolume. Algal density was correlated with algal biovolume in samples from riffles $(r=0.59, \mathrm{p}<0.05)$ but not in samples from pools.

The highest values of algal density in riffle samples occurred in the Tongue River drainage basin, at sites such as Hanging Woman Creek (sites T12 and T13) and the main-stem
Tongue River (sites T5 and T10). Those sites also contained relatively high algal biovolume, as did Goose Creek (site T2) and Tongue River at site T14 (table 10). The lowest values of density from riffles occurred at various sites such as the mainstem Powder River (site P4), Little Powder River (site P16), and Pumpkin Creek (site T19). The lowest values of biovolume from riffle samples occurred on the main-stem Powder River (sites P3, P4, P5, P8, P9, P11, and P12), which include the middle reach. Algal communities in pools tended to have moderate densities and low biovolume values compared to communities in riffles.

\section{Composition}

Diatoms (Bacillariophyta) dominated the algal communities in terms of taxa richness, but green algae (Chlorophyta) and blue-green algae (Cyanophyta, cyanobacteria) dominated in terms of relative abundance (table 11). Algal taxa richness in both riffle and pool habitats generally was higher in 2005 than in 2007. For example, the 2005 riffle samples contained a mean of 66 taxa per sample, of which 57 were diatoms, whereas in 2007, riffle samples contained a mean of 41 taxa per sample, of which 31 were diatoms. Compared to the riffle samples, the pool samples contained similar numbers for sample taxa richness and diatom taxa richness, as well as similar differences in taxa richness between 2005 and 2007. The green and blue-green algae each averaged a few taxa per sample in riffles and pools. Among all samples, green algae averaged 26 percent of the relative abundance in 2005 and 67 percent in 2007; blue-green algae averaged 60 percent of the relative abundance in 2005 and 17 percent in 2007. Other types of algae that were less commonly identified in the algal communities included euglenoids (Euglenophyta), yellow-green algae (Chrysophyta), cryptomonads (Cryptophyta), dinoflagellates (Pyrrhophyta), and red algae (Rhodophyta). 
Table 11. Algal community metrics for streams in the Powder River structural basin, Wyoming and Montana, 2005 and 2007.

[Shaded cells indicate main-stem sampling sites on the Tongue River or Powder River. \%, percent; NA, not available]

\begin{tabular}{|c|c|c|c|c|c|c|c|c|c|c|c|c|c|c|}
\hline \multirow[b]{2}{*}{$\begin{array}{c}\text { Site } \\
\text { number } \\
\text { (fig. 1) }\end{array}$} & \multirow[b]{2}{*}{ Abbreviated site name } & \multirow[b]{2}{*}{ Habitat } & \multirow[b]{2}{*}{$\begin{array}{c}\text { Sample } \\
\text { date }\end{array}$} & \multicolumn{7}{|c|}{ Taxa richness and relative abundance } & \multicolumn{4}{|c|}{ Community metrics } \\
\hline & & & & $\begin{array}{l}\text { Sample } \\
\text { taxa } \\
\text { richness }\end{array}$ & $\begin{array}{c}\text { Diatom } \\
\text { taxa } \\
\text { richness }\end{array}$ & $\begin{array}{c}\text { Diatom } \\
\text { abundance } \\
(\%)\end{array}$ & $\begin{array}{c}\text { Blue-green } \\
\text { algae taxa } \\
\text { richness }\end{array}$ & $\begin{array}{c}\text { Blue-green } \\
\text { algae } \\
\text { abundance } \\
(\%)\end{array}$ & $\begin{array}{c}\text { Green } \\
\text { algae taxa } \\
\text { richness }\end{array}$ & $\begin{array}{c}\text { Green } \\
\text { algae } \\
\text { abundance } \\
(\%) \\
\end{array}$ & $\begin{array}{c}\text { Ses- } \\
\text { tonic } \\
(\%)\end{array}$ & $\begin{array}{l}\text { Eutro- } \\
\text { phic } \\
(\%)\end{array}$ & $\begin{array}{c}\text { Motile } \\
(\%)\end{array}$ & $\begin{array}{l}\text { Nitrogen } \\
\text { fixers } \\
(\%)\end{array}$ \\
\hline R1 & Upper Rosebud Creek & Pool & $7 / 9 / 2007$ & 87 & 67 & 46 & 5 & 15 & 10 & 17 & 28 & 51 & 38 & 2 \\
\hline R2 & Rosebud Creek at mouth & Pool & $7 / 23 / 2007$ & 41 & 28 & 15 & 2 & 2 & 7 & 46 & 91 & 56 & 12 & 0 \\
\hline \multirow[t]{2}{*}{$\mathrm{T} 1$} & Tongue River at Monarch & Riffle & $8 / 15 / 2005$ & 43 & 34 & 2 & 4 & 64 & 5 & 34 & NA & NA & NA & NA \\
\hline & & Riffle & $8 / 29 / 2007$ & 40 & 23 & 13 & 4 & 54 & 8 & 19 & 82 & 73 & 10 & 2 \\
\hline \multirow[t]{2}{*}{$\mathrm{T} 2$} & Goose Creek & Riffle & $8 / 17 / 2005$ & 49 & 39 & 6 & 5 & 51 & 5 & 42 & NA & NA & NA & NA \\
\hline & & Riffle & $9 / 11 / 2007$ & 54 & 43 & 54 & 4 & 34 & 4 & 4 & 38 & 69 & 17 & 18 \\
\hline $\mathrm{T} 3$ & Upper Youngs Creek & Riffle & $6 / 21 / 2007$ & 51 & 42 & 6 & 1 & $<1$ & 5 & 33 & 33 & 37 & 2 & 0 \\
\hline $\mathrm{T} 4$ & Youngs Creek at mouth & Pool & $6 / 21 / 2007$ & 59 & 53 & 1 & 2 & 4 & 1 & 92 & 98 & 93 & 3 & 0 \\
\hline T5 & Tongue River below Youngs Creek & Riffle & $8 / 29 / 2007$ & 53 & 42 & 15 & 3 & 33 & 6 & 50 & 78 & 82 & 11 & 3 \\
\hline T6 & Upper Squirrel Creek & Pool & $7 / 11 / 2007$ & 21 & 17 & 1 & 2 & 3 & 1 & 54 & 0 & 0 & 82 & 0 \\
\hline $\mathrm{T} 7$ & Squirrel Creek at mouth & Riffle & $7 / 12 / 2007$ & 45 & 29 & 16 & 6 & 17 & 7 & 65 & 80 & 75 & 6 & 3 \\
\hline \multirow[t]{2}{*}{$\mathrm{T} 8$} & Prairie Dog Creek & Pool & $8 / 16 / 2005$ & 95 & 82 & 8 & 5 & 58 & 6 & 31 & NA & NA & NA & NA \\
\hline & & Pool & 9/11/2007 & 66 & 56 & 10 & 3 & 12 & 5 & 67 & 89 & 83 & 17 & 0 \\
\hline \multirow[t]{2}{*}{$\mathrm{T} 9$} & Tongue River at State line & Riffle & 9/14/2005 & 73 & 58 & 44 & 5 & 37 & 9 & 19 & NA & NA & NA & NA \\
\hline & & Riffle & $8 / 28 / 2007$ & 63 & 49 & 17 & 6 & 72 & 6 & 7 & 31 & 82 & 11 & 5 \\
\hline T10 & Tongue River above Hanging Woman Creek & Riffle & $8 / 28 / 2007$ & 34 & 24 & 11 & 2 & 17 & 7 & 72 & 77 & 87 & 8 & 5 \\
\hline T11 & Upper Hanging Woman Creek & Riffle & $7 / 11 / 2007$ & 27 & 13 & 16 & 4 & 17 & 6 & 63 & 62 & 83 & 11 & 0 \\
\hline $\mathrm{T} 12$ & Middle Hanging Woman Creek & Riffle & $7 / 10 / 2007$ & 66 & 55 & 32 & 4 & 15 & 6 & 52 & 28 & 77 & 11 & 8 \\
\hline $\mathrm{T} 13$ & Hanging Woman Creek at mouth & Riffle & $7 / 10 / 2007$ & 63 & 50 & 11 & 4 & 27 & 5 & 56 & 68 & 73 & 17 & 0 \\
\hline T14 & Tongue River at Birney Day School & Riffle & $8 / 27 / 2007$ & 34 & 21 & 8 & 3 & 20 & 8 & 70 & 88 & 88 & 3 & 1 \\
\hline $\mathrm{T} 15$ & Upper Otter Creek & Pool & $6 / 20 / 2007$ & 73 & 64 & 5 & 2 & 4 & 6 & 90 & 96 & 95 & 3 & 0 \\
\hline T16 & Middle Otter Creek & Riffle & $6 / 20 / 2007$ & 72 & 63 & 2 & 4 & 17 & 4 & 22 & 26 & 26 & 11 & 0 \\
\hline T17 & Otter Creek at mouth & Pool & 6/19/2007 & 60 & 48 & 4 & 3 & 14 & 3 & 63 & 96 & 78 & 19 & 0 \\
\hline T18 & Tongue River below Brandenberg Bridge & Riffle & $8 / 27 / 2007$ & 60 & 42 & 15 & 6 & 15 & 7 & 63 & 83 & 78 & 12 & 0 \\
\hline T19 & Pumpkin Creek & Riffle & $6 / 18 / 2007$ & 26 & 17 & 1 & 4 & 20 & 1 & 77 & 99 & 97 & 3 & 0 \\
\hline \multirow[t]{2}{*}{ P1 } & Powder River above Salt Creek & Riffle & $7 / 20 / 2005$ & 69 & 59 & 3 & 5 & 77 & 5 & 20 & NA & NA & NA & NA \\
\hline & & Riffle & $8 / 30 / 2007$ & 33 & 23 & 5 & 3 & 19 & 4 & 71 & 73 & 69 & 10 & 0 \\
\hline \multirow[t]{2}{*}{ P2 } & Powder River below Salt Creek & Riffle & $7 / 21 / 2005$ & 42 & 34 & 29 & 4 & 41 & 3 & 30 & NA & NA & NA & NA \\
\hline & & Riffle & $8 / 30 / 2007$ & 41 & 29 & 1 & 2 & 18 & 5 & 75 & 97 & 92 & 5 & 0 \\
\hline \multirow[t]{2}{*}{ P3 } & Powder River below Willow Creek & Riffle & $7 / 19 / 2005$ & 63 & 53 & 3 & 4 & 77 & 6 & 20 & NA & NA & NA & NA \\
\hline & & Riffle & $8 / 24 / 2007$ & 27 & 22 & $<1$ & 1 & 37 & 2 & 62 & 100 & 99 & 1 & 0 \\
\hline \multirow[t]{2}{*}{ P4 } & Powder River below Burger Draw & Riffle & $7 / 22 / 2005$ & 56 & 47 & 26 & 3 & 25 & 6 & 49 & NA & NA & NA & NA \\
\hline & & Riffle & $8 / 22 / 2007$ & 1 & 0 & 0 & 0 & 0 & 1 & 100 & 100 & 100 & 0 & 0 \\
\hline \multirow[t]{2}{*}{ P5 } & Powder River above Crazy Woman Creek & Riffle & $7 / 13 / 2005$ & 92 & 81 & 1 & 3 & 65 & 6 & 33 & NA & NA & NA & NA \\
\hline & & Riffle & $8 / 23 / 2007$ & 1 & 0 & 0 & 0 & 0 & 1 & 100 & 100 & 100 & 0 & 0 \\
\hline \multirow[t]{2}{*}{ P6 } & Crazy Woman Creek below I-90 & Riffle & $7 / 11 / 2005$ & 115 & 106 & 11 & 4 & 54 & 4 & 34 & NA & NA & NA & NA \\
\hline & & Riffle & $8 / 20 / 2007$ & 69 & 58 & 4 & 4 & 27 & 5 & 68 & 90 & 89 & 9 & 0 \\
\hline \multirow[t]{2}{*}{ P7 } & Crazy Woman Creek near mouth & Riffle & $7 / 12 / 2005$ & 78 & 72 & $<1$ & 3 & 92 & 3 & 8 & NA & NA & NA & NA \\
\hline & & Riffle & $8 / 21 / 2007$ & 72 & 59 & 2 & 6 & 17 & 5 & 79 & 96 & 91 & 4 & 1 \\
\hline
\end{tabular}


Table 11. Algal community metrics for streams in the Powder River structural basin, Wyoming and Montana, 2005 and 2007.-Continued

[Shaded cells indicate main-stem sampling sites on the Tongue River or Powder River. \%, percent; NA, not available]

\begin{tabular}{|c|c|c|c|c|c|c|c|c|c|c|c|c|c|c|}
\hline \multirow[b]{2}{*}{$\begin{array}{c}\text { Site } \\
\text { number } \\
\text { (fig. 1) }\end{array}$} & \multirow[b]{2}{*}{ Abbreviated site name } & \multirow[b]{2}{*}{ Habitat } & \multirow[b]{2}{*}{$\begin{array}{c}\text { Sample } \\
\text { date }\end{array}$} & \multicolumn{7}{|c|}{ Taxa richness and relative abundance } & \multicolumn{4}{|c|}{ Community metrics } \\
\hline & & & & $\begin{array}{c}\text { Sample } \\
\text { taxa } \\
\text { richness }\end{array}$ & $\begin{array}{l}\text { Diatom } \\
\text { taxa } \\
\text { richness }\end{array}$ & $\begin{array}{c}\text { Diatom } \\
\text { abundance } \\
(\%)\end{array}$ & $\begin{array}{c}\text { Blue-green } \\
\text { algae taxa } \\
\text { richness }\end{array}$ & $\begin{array}{c}\text { Blue-green } \\
\text { algae } \\
\text { abundance } \\
(\%)\end{array}$ & $\begin{array}{l}\text { Green } \\
\text { algae taxa } \\
\text { richness }\end{array}$ & $\begin{array}{c}\text { Green } \\
\text { algae } \\
\text { abundance } \\
(\%)\end{array}$ & $\begin{array}{c}\text { Ses- } \\
\text { tonic } \\
(\%)\end{array}$ & $\begin{array}{c}\text { Eutro- } \\
\text { phic } \\
(\%)\end{array}$ & $\begin{array}{c}\text { Motile } \\
(\%)\end{array}$ & $\begin{array}{c}\text { Nitrogen } \\
\text { fixers } \\
(\%)\end{array}$ \\
\hline \multirow[t]{2}{*}{ P8 } & Powder River below Crazy Woman Creek & Riffle & $7 / 23 / 2005$ & 68 & 57 & 1 & 6 & 81 & 5 & 18 & NA & NA & NA & NA \\
\hline & & Riffle & $8 / 21 / 2007$ & 46 & 37 & $<1$ & 1 & 1 & 4 & 96 & 97 & 95 & 2 & 0 \\
\hline \multirow[t]{2}{*}{ P9 } & Powder River above Clear Creek & Riffle & $7 / 24 / 2005$ & 96 & 82 & 9 & 5 & 58 & 8 & 32 & NA & NA & NA & NA \\
\hline & & Riffle & $7 / 26 / 2007$ & 6 & 0 & 0 & 0 & 0 & 3 & 98 & 100 & 98 & 2 & 0 \\
\hline \multirow[t]{2}{*}{$\mathrm{P} 10$} & Clear Creek & Riffle & $9 / 13 / 2005$ & 56 & 47 & 22 & 3 & 47 & 5 & 26 & NA & NA & NA & NA \\
\hline & & Riffle & 9/10/2007 & 55 & 42 & 9 & 3 & 15 & 7 & 73 & 83 & 86 & 11 & 0 \\
\hline \multirow{2}{*}{ P11 } & Powder River below Clear Creek & Riffle & $7 / 25 / 2005$ & 58 & 50 & 1 & 4 & 81 & 4 & 18 & NA & NA & NA & NA \\
\hline & & Riffle & $7 / 26 / 2007$ & 1 & 0 & 0 & 0 & 0 & 1 & 100 & 100 & 100 & 0 & 0 \\
\hline \multirow[t]{2}{*}{$\mathrm{P} 12$} & Powder River at Moorhead & Riffle & $7 / 26 / 2005$ & 79 & 69 & 3 & 5 & 73 & 4 & 23 & NA & NA & NA & NA \\
\hline & & Riffle & $7 / 26 / 2007$ & 5 & 0 & 0 & 2 & 1 & 2 & 92 & 100 & 93 & 7 & 0 \\
\hline $\mathrm{P} 13$ & Powder River at Broadus & Riffle & $7 / 25 / 2007$ & 46 & 35 & 3 & 2 & 14 & 6 & 79 & 87 & 83 & 14 & 0 \\
\hline \multirow[t]{2}{*}{ P14 } & Little Powder River at Highway 59 & Pool & $6 / 14 / 2005$ & 56 & 51 & 56 & 3 & 34 & 2 & 10 & NA & NA & NA & NA \\
\hline & & Riffle & $8 / 8 / 2007$ & 43 & 34 & 10 & 3 & 19 & 3 & 58 & 83 & 79 & 17 & 0 \\
\hline \multirow[t]{2}{*}{ P15 } & Little Powder River above Dry Creek & Riffle & $6 / 13 / 2005$ & 25 & 20 & $<1$ & 3 & 90 & 2 & 8 & NA & NA & NA & NA \\
\hline & & Riffle & $8 / 7 / 2007$ & 37 & 27 & 3 & 4 & 15 & 3 & 75 & 95 & 87 & 12 & 0 \\
\hline P16 & Little Powder River at Biddle & Riffle & 8/9/2007 & 29 & 17 & 1 & 5 & 11 & 6 & 87 & 93 & 91 & 7 & 0 \\
\hline P17 & Powder River below Little Powder River & Riffle & $7 / 25 / 2007$ & 85 & 73 & 4 & 2 & 9 & 6 & 84 & 95 & 92 & 5 & 0 \\
\hline P18 & Powder River near Locate & Riffle & $7 / 24 / 2007$ & 48 & 38 & 6 & 4 & 35 & 3 & 51 & 91 & 82 & 14 & 12 \\
\hline \multirow[t]{2}{*}{$\mathrm{C} 1$} & Porcupine Creek & Pool & $6 / 15 / 2005$ & 80 & 74 & 25 & 3 & 49 & 3 & 26 & NA & NA & NA & NA \\
\hline & & Pool & $6 / 12 / 2007$ & 47 & 34 & 5 & 3 & 27 & 6 & 65 & 93 & 91 & 7 & 0 \\
\hline \multirow[t]{2}{*}{$\mathrm{C} 2$} & Antelope Creek & Pool & $6 / 8 / 2005$ & 60 & 47 & 26 & 3 & 30 & 8 & 43 & NA & NA & NA & NA \\
\hline & & Pool & $6 / 14 / 2007$ & 44 & 34 & 2 & 3 & 9 & 4 & 87 & 97 & 95 & 5 & 0 \\
\hline \multirow[t]{2}{*}{$\mathrm{C} 3$} & Cheyenne River near Dull Center & Riffle & $6 / 27 / 2005$ & 56 & 48 & 9 & 3 & 60 & 5 & 31 & NA & NA & NA & NA \\
\hline & & Pool & $6 / 14 / 2007$ & 56 & 47 & 4 & 2 & 23 & 3 & 69 & 96 & 93 & 7 & 0 \\
\hline \multirow[t]{2}{*}{$\mathrm{C} 4$} & Little Thunder Creek & Riffle & $6 / 22 / 2005$ & 66 & 61 & 1 & 4 & 98 & 1 & 1 & NA & NA & NA & NA \\
\hline & & Pool & $6 / 13 / 2007$ & 52 & 46 & 4 & 3 & 15 & 2 & 78 & 94 & 96 & 7 & 4 \\
\hline \multirow[t]{2}{*}{$\mathrm{C} 5$} & Black Thunder Creek & Pool & $6 / 7 / 2005$ & 74 & 59 & 11 & 4 & 40 & 9 & 48 & NA & NA & NA & NA \\
\hline & & Pool & $6 / 13 / 2007$ & 17 & 11 & $<1$ & 2 & 4 & 2 & 92 & 99 & 95 & 4 & 0 \\
\hline \multirow[t]{2}{*}{ C6 } & Cheyenne River near Spencer & Riffle & $6 / 21 / 2005$ & 74 & 67 & 17 & 3 & 50 & 2 & 32 & NA & NA & NA & NA \\
\hline & & Riffle & $6 / 15 / 2007$ & 26 & 18 & 6 & 2 & 15 & 4 & 78 & 94 & 93 & 2 & 0 \\
\hline \multirow[t]{2}{*}{ B1 } & Belle Fourche River & Riffle & $6 / 29 / 2005$ & 69 & 64 & 9 & 3 & 78 & 2 & 13 & NA & NA & NA & NA \\
\hline & & Pool & $6 / 12 / 2007$ & 51 & 40 & 4 & 2 & 19 & 4 & 74 & 92 & 91 & 7 & 0 \\
\hline \multirow[t]{2}{*}{ B2 } & Caballo Creek & Pool & $6 / 28 / 2005$ & 86 & 77 & 33 & 4 & 44 & 5 & 23 & NA & NA & NA & NA \\
\hline & & Pool & $6 / 11 / 2007$ & 39 & 30 & 2 & 4 & 12 & 2 & 83 & 95 & 92 & 6 & 0 \\
\hline
\end{tabular}


The taxonomic composition of the algal communities showed similarities within river drainage basins and by habitat. NMDS ordinations of the Bray-Curtis similarity coefficients for algal taxonomic data from 2007 (fig. 11) and 2005 (Peterson and others, 2009) indicated two general groups of sites corresponding with riffle samples: (1) a Tongue River drainage basin group that included sites on the main stem, Tongue River plains tributaries, and Clear Creek; and (2) a Powder River drainage basin group that included sites on the main-stem Powder River, Crazy Woman Creek, and the Little Powder River. Samples collected from pool habitats in various drainage basins formed a third group of sites.

Algal communities at sites in the Tongue River drainage basin group contained an average of 50 taxa per sample in 2007. Taxa richness in the Tongue River drainage basin group was high compared to the Powder River drainage basin group and similar to the pool group (fig. 12). Green algae tended to be the most abundant algae in both the Tongue River and Powder River drainage basin groups, but the Tongue River drainage basin group generally contained more blue-green algae and less green algae than the Powder River drainage basin group. The most abundant taxa in the Tongue River drainage basin group were Aphanocapsa and Oscillatoria (blue-green algae) and Chlorella (green algae). The proportion of algae that are capable of fixing atmospheric nitrogen tended to be low throughout the study area but was highest in the Tongue River drainage basin group (fig. 12). The relative abundance of nitrogen fixers ranged from 0 percent at most sites to 18 percent in Goose Creek (site T2; table 11). The ability to fix atmospheric nitrogen gives a competitive edge to nitrogen fixers when dissolved-nitrogen concentrations are low or ratios of nitrogen to phosphorus are low (Porter, 2008). Given that Goose Creek receives treated sewage effluent from the city of Sheridan (Wyoming Department of Environmental Quality, 2009), the proportion of nitrogen fixers at site $\mathrm{T} 2 \mathrm{might}$ be a reflection of low ratios of nitrogen to phosphorus. The chlorophyll- $a$ concentration in Goose Creek in 2007 was relatively high at $44.1 \mathrm{mg} / \mathrm{m}^{2}$ (table 10). In 2005, the relative abundance of nitrogen fixers and the chlorophyll- $a$ concentration at Goose Creek were comparatively low (Peterson and others, 2009).

Taxa richness of algal communities in the Powder River drainage basin group in 2007 ranged from 1 to 85 taxa (table 11). The green alga Chlorella composed 92-100 percent

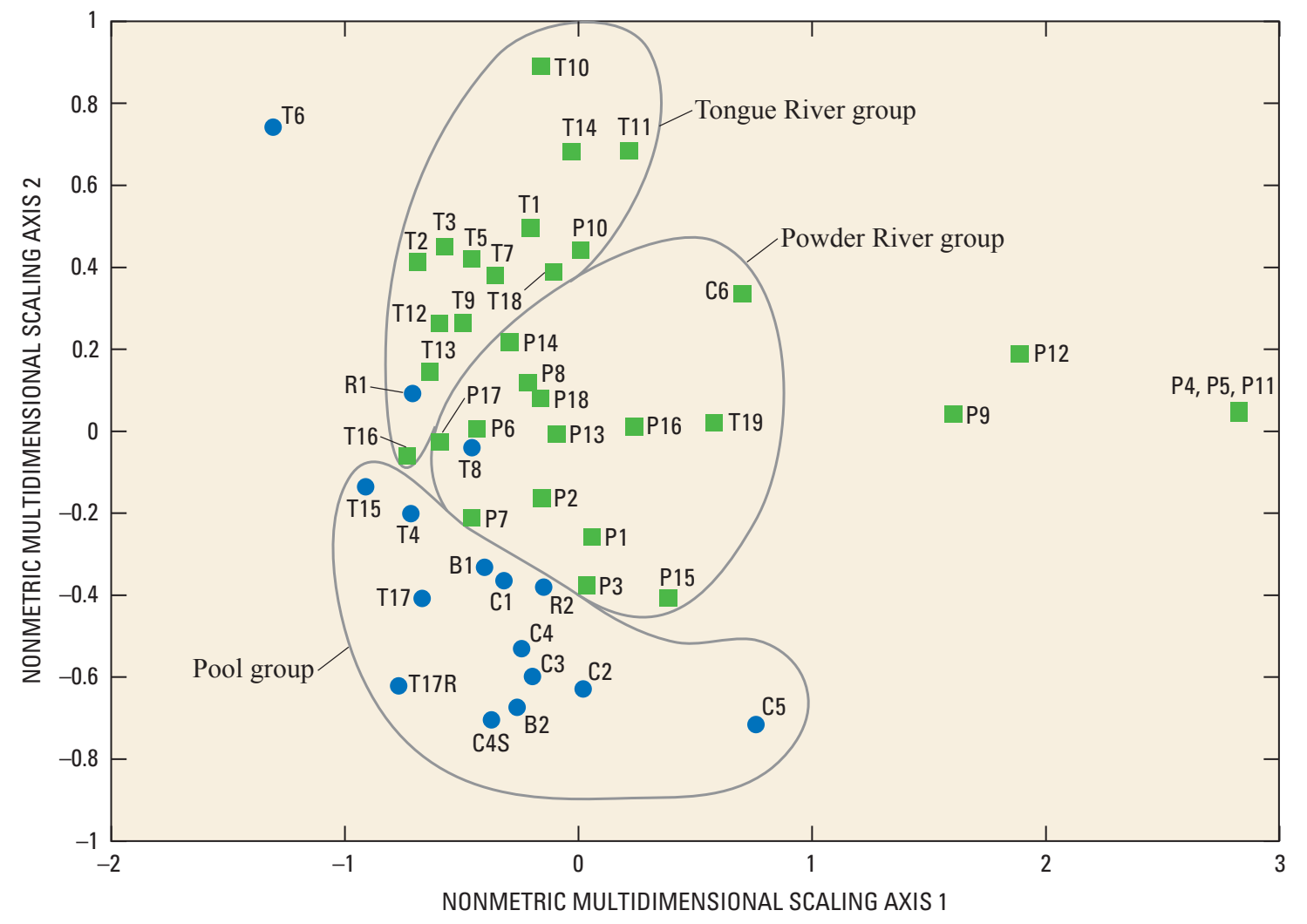

EXPLANATION

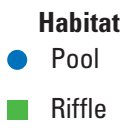

Figure 11. Similarities of algal community taxonomic composition within drainage basins and within habitats, depicted by nonmetric multidimensional scaling ordination, Powder River structural basin, Wyoming and Montana, 2007. 

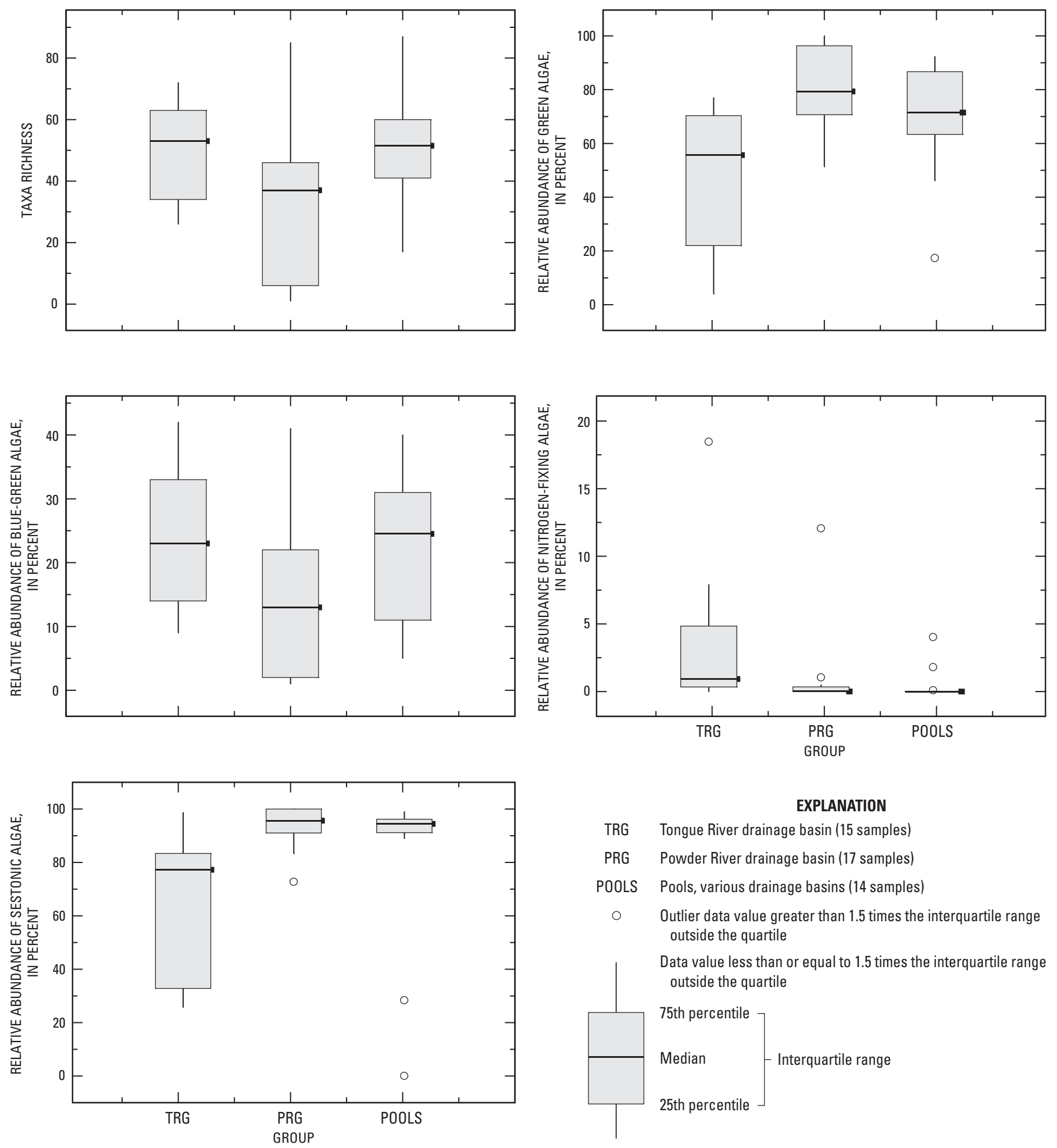

Figure 12. Algal community composition by drainage basin and habitat, Powder River structural basin, 2007. 
of the algal abundance at sites P4, P5, P9, P11, and P12 in 2007, which includes the middle reach (sites P3-P5 and P8), causing these sites to be outliers from the NMDS ordination of the Powder River drainage basin group (fig. 11). For comparison, in 2005, the algal communities at sites P2 and P4 on the mainstem Powder River were dominated by a single species, the diatom Achnanthes minutissima (Peterson and others, 2009). Low algal taxa richness can be an indicator of effects to the community if sensitive species are stressed by natural or other factors (Barbour and others, 1999). The relative abundance of Achnanthes minutissima may increase in response to metal toxicity, nutrient enrichment, scouring, or grazing (Bahls, 1993). Chlorella is a cosmopolitan sestonic algae (planktonic or suspended) (Porter, 2008), which helps explain the generally higher relative abundance of sestonic algae in the Powder River drainage basin group than in the Tongue River drainage basin group (fig. 12). The dominance of sestonic algae might result from the influence of pools and slow-moving reaches upstream from the sample-collection points, as indicated by the similarities between the Powder River and pool groups (fig. 12). The relative abundance of eutrophic algae, which are either tolerant of or dependent on high nutrient concentrations, tended to be higher in the Powder River drainage basin group (average of 91 percent) than in the Tongue River drainage basin group (average of 74 percent).

The pool group contained sites from multiple river drainage basin basins; the common variable among these sites was that algae samples were collected from pools rather than riffles. Algal communities were dominated by green algae, including Chlorella, and blue-green algae such as Aphanocapsa. The relative abundance of sestonic algae was high in the pool group (fig. 12), consistent with the depositional habitat from which the samples were collected.

\section{Fish Communities}

Fish communities were sampled at 12 sites in the Tongue River drainage basin and at 11 sites in the Powder River drainage basin in 2008, and results for these sites are presented along with results from previous fish community sampling reported by Peterson and others (2009). Results for sites that were sampled previously (Peterson and others, 2009) but not in 2008 are not included in this report.

\section{Tongue River Drainage Basin}

Fish communities were sampled at 12 of the 19 sites on the main-stem Tongue River and Tongue River tributaries during 2005-06 and 2008 (table 12), except for Squirrel Creek at mouth (site T7), which was not sampled in 2006 because the sample reach was dry. The remaining seven sites in the Tongue River drainage basin were sampled in 2005-06 but not in 2008; results for these seven sites were presented previously by Peterson and others (2009) and are not described in this report.

\section{Community Composition}

Twenty-nine species of fish were identified in samples collected from the Tongue River drainage basin during 2005-06 and 2008 (table 12). More than one-half of the species (15) identified in the Tongue River drainage basin were native, including seven minnows (Cyprinidae), five suckers (Catostomidae), two catfish (Ictaluridae), and one sauger (Percidae; table 13). Introduced fish species included eight sunfishes (Centrarchidae), three minnows, two catfishes, and yellow perch (Percidae).

Smallmouth bass accounted for 28 percent of the relative abundance of fish captured in the main-stem Tongue River (fig. 13), with white sucker (11 percent), yellow bullhead (11 percent), and rock bass (10 percent) also abundant. In contrast, smallmouth bass composed only 9 percent of the fish captured in the tributaries to the Tongue River (fig. 13). Fathead minnow were the most common fish in the tributaries, where they composed 42 percent of the relative abundance of fish captured. Fathead minnow were relatively uncommon in the main-stem Tongue River, where they were among 16 of 23 species that composed 10 percent of the other fish captured (fig. 13). Fish communities of the main-stem Tongue River also contained yellow bullhead (11 percent; fig. 13), common carp ( 8 percent), shorthead redhorse ( 8 percent), and spottail shiner ( 8 percent) that were uncommon in the tributaries.

Fish species richness was greatest at sampling sites on the main-stem Tongue River. Samples from Tongue River at State line (T9) contained 18 species in 2005, 17 species in 2006, and 14 species in 2008 (table 12). Reduction in the number of species captured for each sampling year can be from normal year-to-year variation, increased turbidity (table 3), changes in sampling efficiency, or a combination of these and unknown factors. Sampling on the Tongue River below Youngs Creek (site T5), Tongue River above Hanging Woman Creek (site T10), and Tongue River at Birney Day School (site T14) resulted in the collection of 10 to 14 species each year. The Tongue River Reservoir, on the main stem between sites T9 and T10 (fig. 1), appears to affect the fish community of the river. Open-water species, such as spottail shiner and yellow perch (Baxter and Stone, 1995), were identified only at sites upstream from the reservoir at the Tongue River below Youngs Creek (site T5), Tongue River at State line (site T9), and Prairie Dog Creek (site T8). Black crappie and white crappie, also known as open-water species, were found in small numbers upstream and downstream from Tongue River Reservoir. Sauger was collected at Tongue River at State line (site T9) in 2008 but was not collected at any other sampling site in the Tongue River drainage basin. Mountain sucker was collected only at Upper Youngs Creek (site T3) in 2005 and 2008.

Tributary streams with relatively large numbers of fish species in all three sampling years were Goose Creek (site T2) and Prairie Dog Creek (site T8). Numbers of fish species collected at Goose Creek (site T2) were 11 in 2005, 8 in 2006, and 10 in 2008. Nine species were collected at Prairie Dog Creek 
Table 12. Fish abundance in samples from the Tongue River drainage basin, Wyoming and Montana, 2005-06 and 2008.

[Shaded cells indicate main-stem sampling sites on the Tongue River]

\begin{tabular}{|c|c|c|c|c|c|c|c|c|c|c|c|c|c|c|}
\hline $\begin{array}{l}\text { Site } \\
\text { number } \\
\text { (fig. 1) }\end{array}$ & Abbreviated site name & $\begin{array}{l}\text { Sample } \\
\text { date }\end{array}$ & $\begin{array}{l}\text { Black } \\
\text { bull- } \\
\text { head }\end{array}$ & $\begin{array}{c}\text { Black } \\
\text { crappie }\end{array}$ & $\begin{array}{l}\text { Blue- } \\
\text { gill }\end{array}$ & $\begin{array}{c}\text { Brassy } \\
\text { minnow }\end{array}$ & $\begin{array}{l}\text { Channel } \\
\text { catfish }\end{array}$ & $\begin{array}{c}\text { Common } \\
\text { carp }\end{array}$ & $\begin{array}{l}\text { Creek } \\
\text { chub }\end{array}$ & $\begin{array}{c}\text { Fat- } \\
\text { head } \\
\text { minnow }\end{array}$ & $\begin{array}{l}\text { Flat- } \\
\text { head } \\
\text { chub }\end{array}$ & $\begin{array}{l}\text { Golden } \\
\text { shiner }\end{array}$ & $\begin{array}{l}\text { Green } \\
\text { sunfish }\end{array}$ & $\begin{array}{l}\text { Lake } \\
\text { chub }\end{array}$ \\
\hline \multirow[t]{2}{*}{$\mathrm{T} 2$} & Goose Creek & $8 / 22 / 2005$ & 3 & 0 & 0 & 0 & 0 & 18 & 0 & 1 & 0 & 0 & 3 & 0 \\
\hline & & $8 / 22 / 2006$ & 15 & 0 & 0 & 0 & 0 & 30 & 0 & 0 & 0 & 0 & 3 & 0 \\
\hline \multirow[t]{3}{*}{$\mathrm{T} 3$} & Upper Youngs Creek & $6 / 15 / 2005$ & 0 & 0 & 0 & 0 & 0 & 0 & 6 & 2 & 0 & 0 & 0 & 0 \\
\hline & & $6 / 28 / 2006$ & 0 & 0 & 0 & 0 & 0 & 0 & 62 & 15 & 0 & 0 & 0 & 0 \\
\hline & & $6 / 26 / 2008$ & 0 & 0 & 0 & 0 & 0 & 0 & 19 & 0 & 0 & 0 & 0 & 0 \\
\hline $\mathrm{T} 4$ & & $6 / 26 / 2008$ & 2 & 0 & 0 & 2 & 0 & 2 & 14 & 1 & 0 & 0 & 2 & 0 \\
\hline \multirow[t]{3}{*}{ T5 } & Tongue River below Youngs Creek & $8 / 29 / 2005$ & 108 & 3 & 0 & 0 & 0 & 43 & 0 & 17 & 0 & 0 & 42 & 0 \\
\hline & & $8 / 24 / 2006$ & 10 & 0 & 0 & 0 & 0 & 129 & 0 & 0 & 4 & 0 & 5 & 0 \\
\hline & & $9 / 11 / 2008$ & 5 & 0 & 0 & 0 & 0 & 5 & 0 & 1 & 0 & 0 & 5 & 0 \\
\hline \multirow[t]{3}{*}{ T6 } & Upper Squirrel Creek & $6 / 16 / 2005$ & 0 & 0 & 0 & 0 & 0 & 0 & 19 & 0 & 0 & 0 & 0 & 0 \\
\hline & & $6 / 29 / 2006$ & 0 & 0 & 0 & 1 & 0 & 0 & 10 & 83 & 0 & 0 & 0 & 0 \\
\hline & & $6 / 25 / 2008$ & 0 & 0 & 0 & 0 & 0 & 1 & 0 & 20 & 0 & 0 & 0 & 134 \\
\hline T8 & & $9 / 10 / 2008$ & 9 & 0 & 0 & 0 & 0 & 0 & 0 & 0 & 1 & 0 & 1 & 0 \\
\hline \multirow[t]{3}{*}{ T9 } & Tongue River at State line & $8 / 25 / 2005$ & 30 & 25 & 4 & 1 & 6 & 89 & 9 & 0 & 2 & 0 & 1 & 0 \\
\hline & & $8 / 28 / 2006$ & 13 & 3 & 0 & 0 & 9 & 99 & 51 & 0 & 3 & 0 & 6 & 0 \\
\hline & & $9 / 10 / 2008$ & 6 & 0 & 0 & 0 & 0 & 8 & 0 & 4 & 0 & 17 & 0 & 0 \\
\hline \multirow[t]{3}{*}{$\mathrm{T} 10$} & $\begin{array}{l}\text { Tongue River above Hanging } \\
\text { Woman Creek }\end{array}$ & $8 / 30 / 2005$ & 0 & 9 & 0 & 0 & 10 & 21 & 0 & 0 & 0 & 0 & 1 & 0 \\
\hline & & $8 / 29 / 2006$ & 0 & 0 & 0 & 0 & 7 & 24 & 0 & 0 & 0 & 0 & 0 & 0 \\
\hline & & $9 / 9 / 2008$ & 0 & 0 & 0 & 0 & 9 & 7 & 0 & 0 & 0 & 0 & 0 & 0 \\
\hline \multirow[t]{3}{*}{ T13 } & Hanging Woman Creek at mouth & $6 / 23 / 2005$ & 0 & 0 & 0 & 0 & 0 & 0 & 0 & 85 & 0 & 0 & 17 & 0 \\
\hline & & $6 / 26 / 2006$ & 0 & 0 & 0 & 0 & 0 & 0 & 0 & 266 & 0 & 0 & 0 & 0 \\
\hline & & $6 / 24 / 2008$ & 0 & 0 & 0 & 0 & 0 & 4 & 0 & 199 & 0 & 130 & 8 & 0 \\
\hline \multirow[t]{2}{*}{ T14 } & Tongue River at Birney Day School & $8 / 31 / 2005$ & 0 & 0 & 0 & 0 & 18 & 35 & 0 & 0 & 0 & 0 & 0 & 0 \\
\hline & & $8 / 30 / 2006$ & 2 & 0 & 0 & 0 & 63 & 35 & 0 & 0 & 0 & 2 & 17 & 0 \\
\hline
\end{tabular}

(site T8) in 2005, and 10 species were collected in 2006 and 2008. The numbers of fish species sampled at Youngs Creek at mouth (site T4), Upper Squirrel Creek (site T6), Hanging Woman Creek at mouth (T13), and Otter Creek at mouth (T17) were somewhat variable from year to year.

With a few exceptions, fish communities sampled in all 3 years appeared to be similar on the basis of species richness. At Squirrel Creek at mouth (site T7), five fish species were collected in 2005 and two species were collected in 2008.

The lack of fish species collected in 2008 may be the result of drought conditions in 2006, indicating fish species have yet to fully repopulate the site. Only one species was identified in 2006 at Hanging Woman Creek at mouth (site T13) presumably because 2006 was a drier year when compared to the other sampling years, and suitable habitat was not present for other species (table 3). Otherwise, the number of fish species found at site T13 increased overall from four species collected in 2005 to seven species collected in 2008. Otter Creek at mouth (site T17) showed a decrease in the number of fish species identified for each year with six species collected in 2005 , five species collected in 2006, and three species collected in 2008.

The total abundance of fish collected at the 12 sites was 3,826 individuals in 2005, 5,705 in 2006, and 2,562 in 2008. Although 2006 was a drier year, the mean number of species collected per site was similar for the 3 years: 8.1 species in $2005,7.7$ species in 2006, and 8.1 species in 2008 . The largest increases in fish abundance from 2005 to 2008 were for sand shiners, fathead minnow, lake chub, and golden shiner. The largest decreases in fish abundance from 2005 to 2008 were for common carp, channel catfish, and white sucker. The difference in abundance between years is probably related to normal year-to-year variation.

\section{Community Structure}

The structure and integrity of the fish community were assessed using the Index of Biotic Integrity (IBI) developed for small prairie streams in Montana (Bramblett and others, 
Table 12. Fish abundance in samples from the Tongue River drainage basin, Wyoming and Montana, 2005-06 and 2008.-Continued

[Shaded cells indicate main-stem sampling sites on the Tongue River]

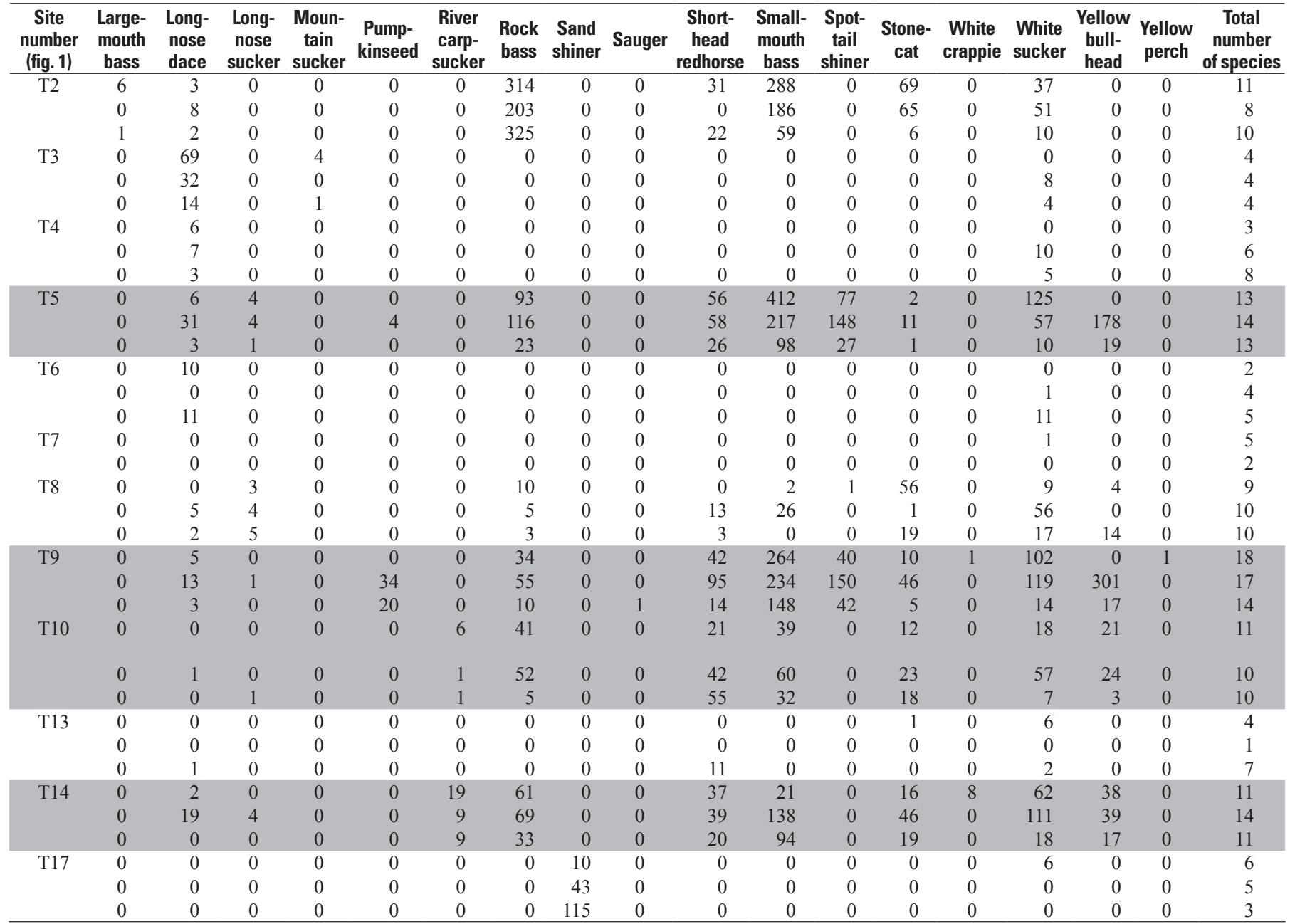

2005). IBI scores consist of 10 metrics, some of which are adjusted by the drainage area. Because the IBI was not intended for use in rivers with large drainage areas, raw and unadjusted fish community metric values were evaluated for the main-stem Tongue River (table 14).

Testing using ANOVA with the Tukey method was performed to determine statistical differences in fish community metrics between sites and by year for the main-stem Tongue River. Results show significant differences $(p<0.05)$ among sites for percentage of invertivorous cyprinid individuals and percentage of native individuals (table 15). The Tongue River Reservoir on the main stem between Tongue River at State line (site T9) and Tongue River above Hanging Woman Creek (site T10) appears to affect the fish communities of the river. As shown in figure 14, the number of benthic invertivorous species and percentage of invertivorous cyprinids were higher upstream from the reservoir than downstream; these metrics are expected to decline as human influence increases (Bramblett and others, 2005). The number of native catostomid and ictalurid species, number of longlived native species, and percentage of native individuals generally were higher downstream than upstream from the the reservoir; these metrics also are expected to decline as human influence increases (Bramblett and others, 2005). The causes are unknown as to why the metrics are inconsistent with regard to human influence upstream and downstream from the reservoir, but might be related to fish migration from Tongue River Reservoir.

Of the small plains streams that were sampled, the fish communities from Youngs Creek (sites T3 and T4) and Squirrel Creek (sites T6 and T7 in 2005) had some of the highest IBI scores in the study area (table 16). The highest observed IBI score of 96 occurred at site T3 on upper Youngs Creek in 2008 (fig. 15). IBI scores from sites on lower Youngs Creek (site T4) and Squirrel Creek (sites T6 and T7 in 2005) also were relatively high and ranged from 66 to 84, 
Table 13. Ecological characteristics of fish species sampled in the Powder River Structural Basin, Wyoming and Montana, 2005-08.

[Modified from Bramblett and others, 2005. Trophic category: IN, invertivore; HB, herbivore; OM, omnivore; IC, invertivore-carnivore; CA, carnivore. Feeding habitat: WC, water column; BE, benthic; GE, generalist. Reproductive class: LO, litho-obligate; TR, tolerant reproductive strategists; --, not determined or not available; General tolerance: INT, intolerant; MOD, moderate; TOL, tolerant. Origin: N, native; I, introduced. mm, millimeters; NA, not applicable because species generally lives less than 3 years]

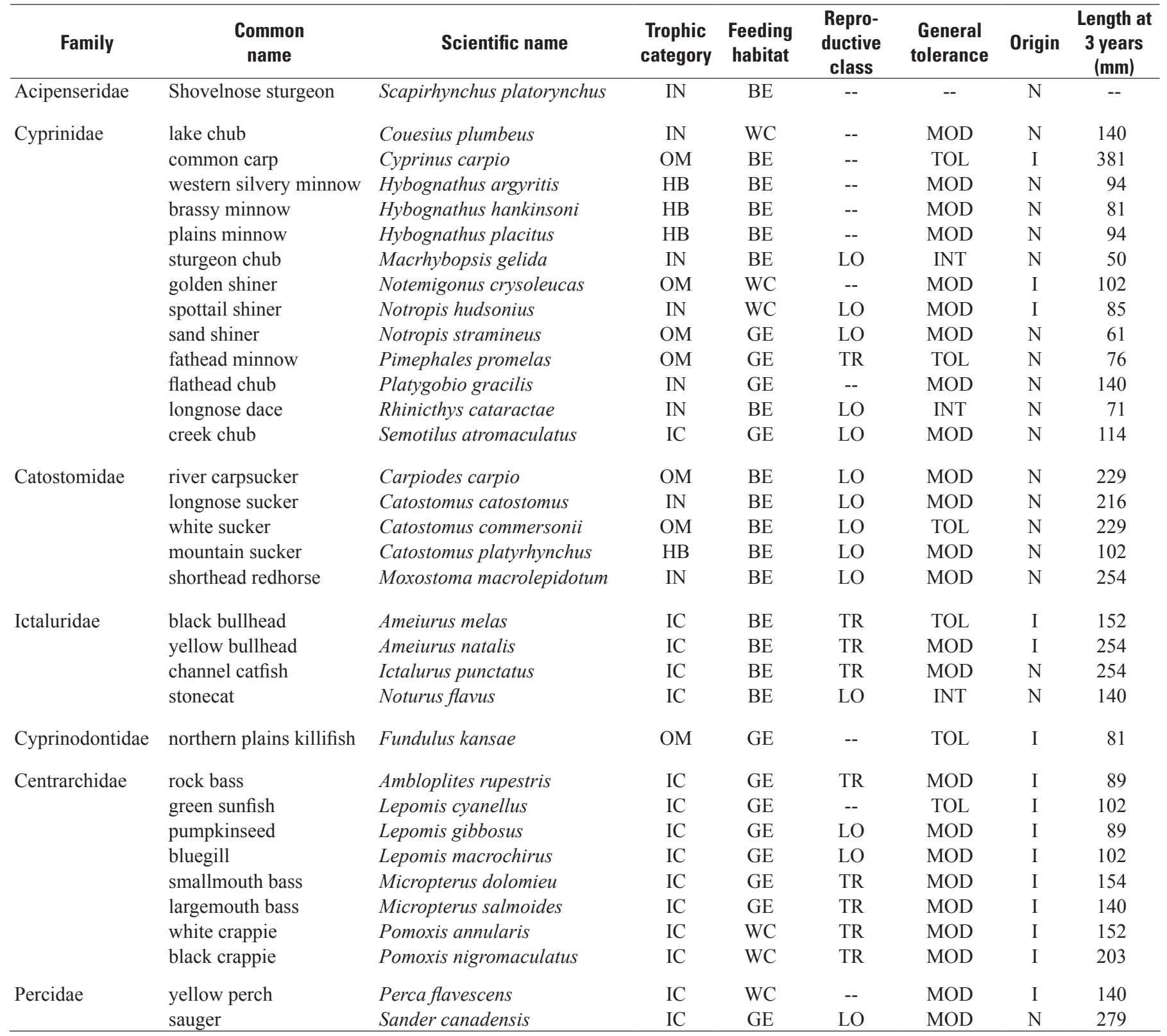

although scores decreased in Youngs Creek from site T3 to site T4 in all 3 years of sampling (table 16). Squirrel Creek at mouth (site T7) had a marked decrease in the IBI score from 2005 to 2008, presumably because the site was dry in 2006 and effects were still being seen in 2008. Goose Creek (site T2) and Otter Creek at mouth (site T17) had downward trends in IBI score from 2005 to 2008 (fig. 15). Prairie Dog Creek (site T8) had IBI scores ranging from 59 to 78 with a mean of 69. Hanging Woman Creek at mouth (site T13) had some of lowest IBI scores ranging from 40 to 46 with a mean of 43 .

\section{Powder River Drainage Basin}

Fish communities were sampled on the main-stem Powder River and Powder River tributaries at 11 of 18 sites during 2004-06 and 2008. The remaining seven sites in the Powder River drainage basin were sampled in 2004-06 but not in 2008; results for these seven sites were presented previously by Peterson and others (2009) and are not described in this report. For sites that had multiple sample dates during 2004-05, the sample date closest to the 2008 sample date was chosen for comparisons between years. 


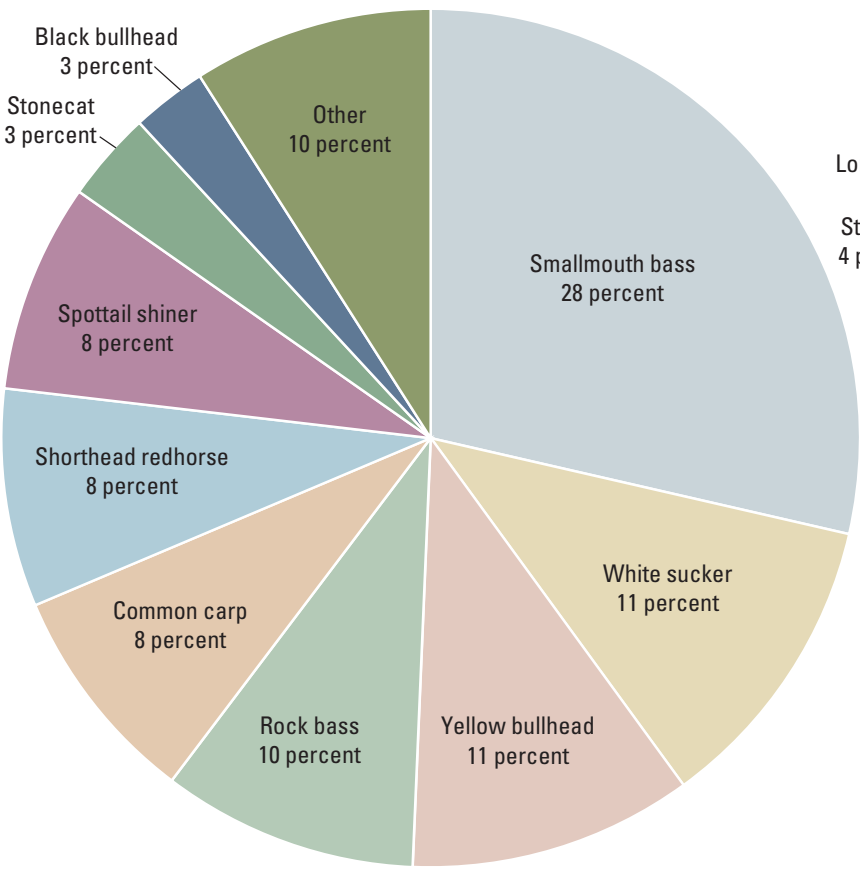

Main-stem Tongue River

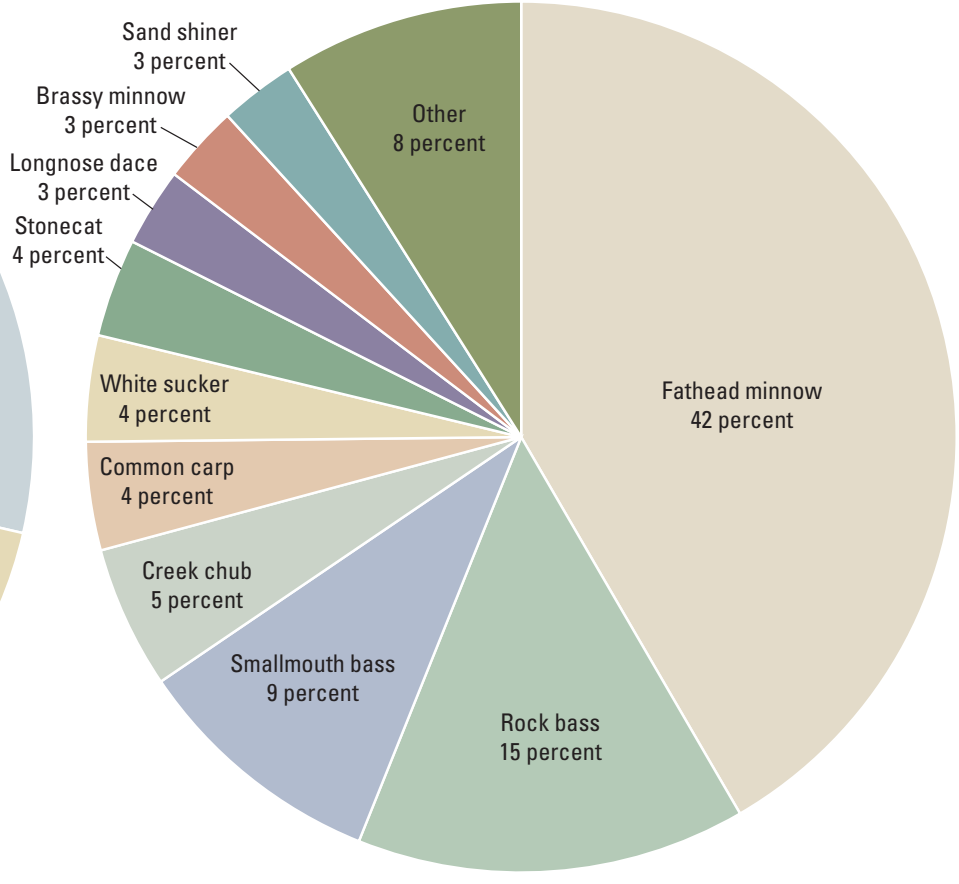

Tongue River tributaries

Figure 13. Relative abundance of fish by species in samples from the Tongue River drainage basin, Wyoming and Montana, 2005-06 and 2008.

\section{Community Composition}

Twenty-four species of fish were identified in samples collected during 2004-06 and 2008 (table 17). Out of the those species identified, 17 were native, including 8 minnows (Cyprinidae), 4 suckers (Catostomidae), 2 catfish (Ictaluridae), 1 goldeye (Hiodontidae), 1 shovelnose sturgeon (Acipenseridae) and 1 sauger (Percidae). Introduced fish species included four sunfishes (Centrarchidae), one minnow, one catfish, and one northern plains killifish (Cyprinodontidae).

Sand shiner were the most common fish in the Powder River drainage basin, accounting for 63 percent of the relative abundance of fish captured in the main-stem Powder River and 48 percent in the tributaries to the Powder River (fig. 16). Flathead chub composed 22 percent of the fish in the main-stem Powder River and 6 percent in the tributaries. Hybognathus spp. composed 5 percent of the fish in the main-stem Powder River but were uncommon in the tributaries, whereas white sucker and rock bass were more common in the tributaries than in the main stem (fig. 16). Of the two species of Hybognathus identified, the plains minnow (H. placitus) was more common than the western silvery minnow (H. argyritis; table 17 ) that is a Wyoming species of concern (Wyoming Game and Fish Department, written commun. 2008). In total, 21 fish species were captured from the main-stem Powder River during 2004-06 and 2008, and 20 species were captured from the tributaries to the Powder River during 2004-06 and 2008.

Fish species richness on the main-stem Powder River tended to be relatively high in the middle reach of the Powder River (sites P3-P5 and P8). Richness was highest at Powder
River downstream from Burger Draw (P4) with 11 species in 2005, 10 species in 2006, and 13 species collected in 2008 (table 17). Other sites on the main-stem Powder River with high species richness were Powder River above Pumpkin Creek (site P3), which contained 8 to 12 species, and Powder River above Crazy Woman Creek (site P5), which contained 7 to 11 species. Four fish species were identified on the main-stem Powder River and not in tributary streams. Bluegill was collected only at Powder River below Burger Draw (site P4) in 2008. Mountain sucker was collected only at Powder River above Pumpkin Creek (site P3) in 2004. Shovelnose sturgeon was collected at Powder River at Broadus (site P13) in 2008. Sturgeon chub, which is a Wyoming species of concern (Wyoming Game and Fish Department, written commun. 2008), was collected at Powder River above Crazy Woman Creek (site P5) in 2006 and Powder River at Moorhead (site P12) in 2006 and 2008.

The tributary stream in the Powder River with relatively large numbers of fish species in 3 sampling years was Clear Creek (site P10) with 14, 14, and 11 species in 2005, 2006, and 2008, respectively. Clear Creek (site P10) was the only tributary where sauger was collected in the 3 years of sampling.

The total abundance of fish collected at the 11 sites was 6,114 in 2004, 9,462 in 2005, 23,786 in 2006, and 2,629 individuals collected in 2008. A possible cause for the large number of fish caught in 2006 is that the drought concentrated the fish in smaller areas, making them easier to catch. 
Table 14. Unadjusted fish community metric values for the main-stem Tongue River, Wyoming and Montana, $2005-06$ and 2008.

[Metric definitions from Bramblett and others (2005)]

\begin{tabular}{|c|c|c|c|c|c|c|c|c|c|c|c|c|}
\hline \multirow[b]{2}{*}{$\begin{array}{c}\text { Site } \\
\text { number } \\
\text { (fig. 1) }\end{array}$} & \multirow[b]{2}{*}{ Abbreviated site name } & \multirow[b]{2}{*}{$\begin{array}{c}\text { Sample } \\
\text { date }\end{array}$} & \multicolumn{5}{|c|}{ Number of } & \multicolumn{5}{|c|}{ Percentage of } \\
\hline & & & $\begin{array}{l}\text { Native } \\
\text { species }\end{array}$ & $\begin{array}{l}\text { Native } \\
\text { families }\end{array}$ & $\begin{array}{c}\text { Native } \\
\text { catostomid } \\
\text { and ictalurid } \\
\text { species }\end{array}$ & $\begin{array}{c}\text { Benthic } \\
\text { invertivorous } \\
\text { species }\end{array}$ & $\begin{array}{l}\text { Native } \\
\text { long-lived } \\
\text { species }\end{array}$ & $\begin{array}{c}\text { Tolerant } \\
\text { individuals }\end{array}$ & $\begin{array}{c}\text { Invertivorous } \\
\text { cyprinid } \\
\text { individuals }\end{array}$ & $\begin{array}{l}\text { Litho-obligate } \\
\text { reproductive } \\
\text { guild } \\
\text { individuals }\end{array}$ & $\begin{array}{c}\text { Tolerant } \\
\text { reproductive } \\
\text { guild } \\
\text { individuals }\end{array}$ & $\begin{array}{c}\text { Native } \\
\text { individuals }\end{array}$ \\
\hline \multirow[t]{3}{*}{ T5 } & $\begin{array}{l}\text { Tongue River below } \\
\text { Youngs Creek }\end{array}$ & $8 / 29 / 2005$ & 6 & 3 & 4 & 3 & 4 & 34 & 8 & 27 & 64 & 21 \\
\hline & & $8 / 24 / 2006$ & 6 & 3 & 4 & 3 & 4 & 21 & 19 & 32 & 54 & 17 \\
\hline & & $9 / 11 / 2008$ & 6 & 3 & 4 & 3 & 8 & 12 & 13 & 30 & 65 & 19 \\
\hline \multirow[t]{3}{*}{ T9 } & Tongue River at State line & $8 / 25 / 2005$ & 8 & 3 & 4 & 2 & 4 & 33 & 8 & 32 & 54 & 27 \\
\hline & & $8 / 28 / 2006$ & 8 & 3 & 5 & 3 & 3 & 19 & 18 & 41 & 50 & 27 \\
\hline & & $9 / 10 / 2008$ & 6 & 4 & 3 & 2 & 8 & 16 & 15 & 32 & 60 & 13 \\
\hline \multirow[t]{3}{*}{$\mathrm{T} 10$} & $\begin{array}{l}\text { Tongue River above } \\
\text { Hanging Woman Creek }\end{array}$ & $8 / 30 / 2005$ & 5 & 3 & 5 & 1 & 5 & 20 & 0 & 29 & 60 & 34 \\
\hline & & $8 / 29 / 2006$ & 6 & 3 & 5 & 2 & 6 & 28 & 0 & 43 & 49 & 45 \\
\hline & & $9 / 9 / 2008$ & 6 & 2 & 6 & 2 & 9 & 10 & 0 & 59 & 36 & 66 \\
\hline \multirow[t]{3}{*}{$\mathrm{T} 14$} & $\begin{array}{l}\text { Tongue River at Birney } \\
\text { Day School }\end{array}$ & $8 / 31 / 2005$ & 6 & 3 & 5 & 2 & 5 & 31 & 1 & 43 & 46 & 49 \\
\hline & & $8 / 30 / 2006$ & 7 & 3 & 6 & 3 & 7 & 28 & 3 & 38 & 52 & 49 \\
\hline & & $9 / 8 / 2008$ & 5 & 2 & 5 & 1 & 9 & 36 & 0 & 27 & 65 & 32 \\
\hline
\end{tabular}

Table 15. Analysis of variance in fish community metrics, main-stem Tongue River and main-stem Powder River, Wyoming and Montana, $2005-08$.

[Significance tested by Tukey method at probablility level less than 0.05; data for Powder River also include samples collected during 2004]

\begin{tabular}{|c|c|c|c|c|}
\hline \multirow[b]{2}{*}{ Metric } & \multicolumn{2}{|c|}{ Main-stem Tongue River } & \multicolumn{2}{|c|}{ Main-stem Powder River } \\
\hline & $\begin{array}{c}\text { Significant differences } \\
\text { between sites }\end{array}$ & $\begin{array}{c}\text { Significant differences } \\
\text { between years }\end{array}$ & $\begin{array}{c}\text { Significant differences } \\
\text { between sites }\end{array}$ & $\begin{array}{c}\text { Significant differences } \\
\text { between years }\end{array}$ \\
\hline Number of native species & None & None & None & None \\
\hline Number of native families & None & None & None & None \\
\hline Number of native catostomid and ictalurid species & None & None & None & None \\
\hline Number of benthic invertivorous species & None & None & None & None \\
\hline Number of native species with long lived individuals & None & None & None & None \\
\hline Percentage of tolerant individuals & None & None & $\mathrm{P} 3$ to $\mathrm{P} 8, \mathrm{P} 11$ to $\mathrm{P} 13$ & None \\
\hline Percentage of invertivorous cyprinid individuals & $\begin{array}{c}\mathrm{T} 10 \text { to } \mathrm{T} 5, \mathrm{~T} 10 \text { to } \mathrm{T} 9, \mathrm{~T} 14 \text { to } \\
\mathrm{T} 5, \mathrm{~T} 14 \text { to } \mathrm{T} 9\end{array}$ & None & None & None \\
\hline Percentage of litho-obligate reproductive guild individuals & None & None & None & None \\
\hline Percentage of tolerant reproductive guild individuals & None & None & None & None \\
\hline Percentage of native individuals & $\begin{array}{c}\text { T10 to } \mathrm{T} 5, \mathrm{~T} 10 \text { to } \mathrm{T} 9, \mathrm{~T} 14 \text { to } \\
\text { T5 }\end{array}$ & None & $\mathrm{P} 11$ to $\mathrm{P} 13$ & None \\
\hline
\end{tabular}



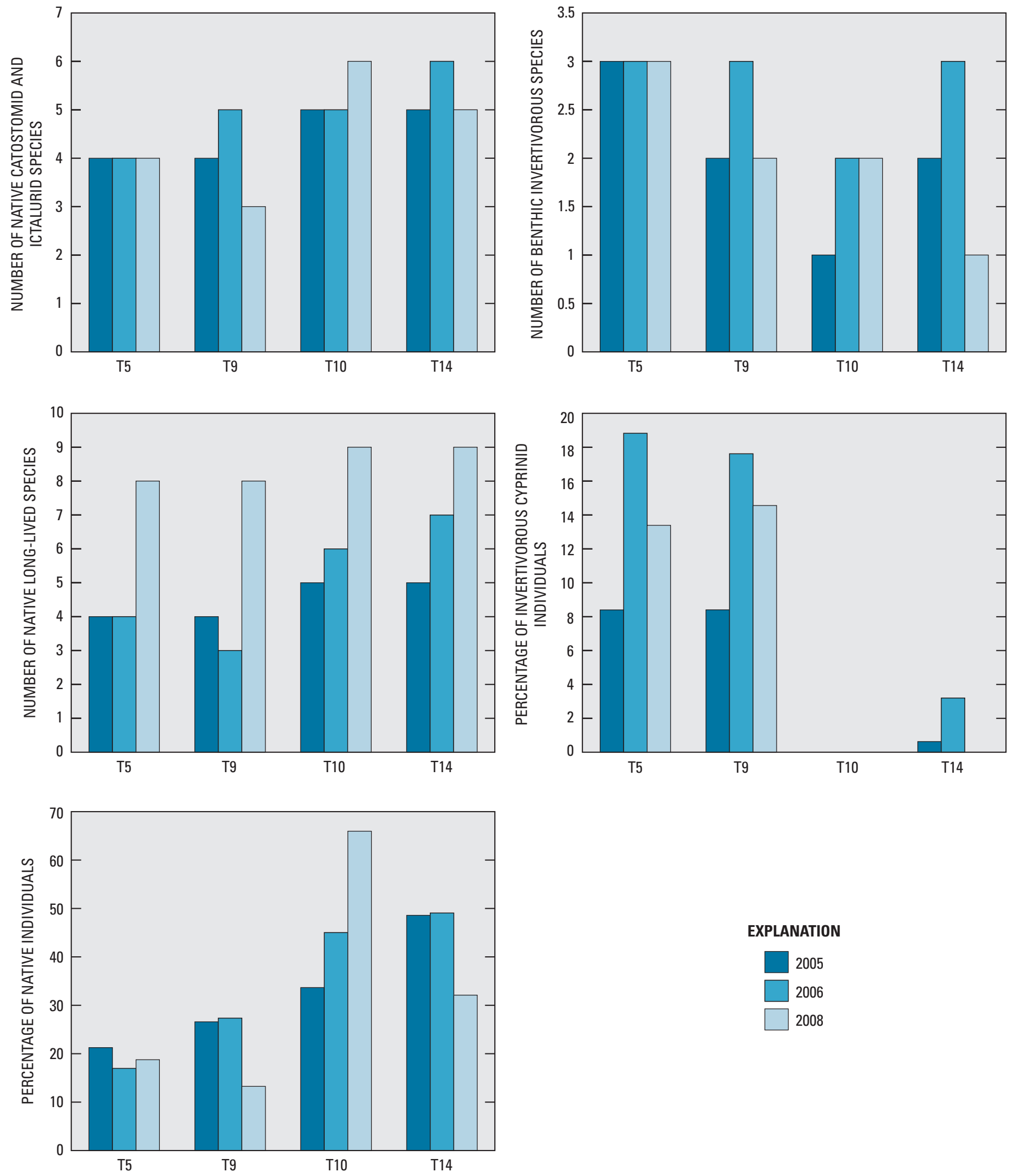

\section{EXPLANATION}

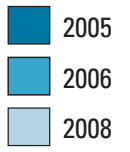

Figure 14. Selected unadjusted fish community metric values for the main-stem Tongue River, Wyoming and Montana, 2005-06 and 2008. 
Table 16. Fish community metric and Index of Biotic Integrity (IBI) scores for the Tongue and Powder River tributaries, Wyoming and Montana, 2005-06 and 2008.

[Metric definitions from Bramblett and others (2005)]

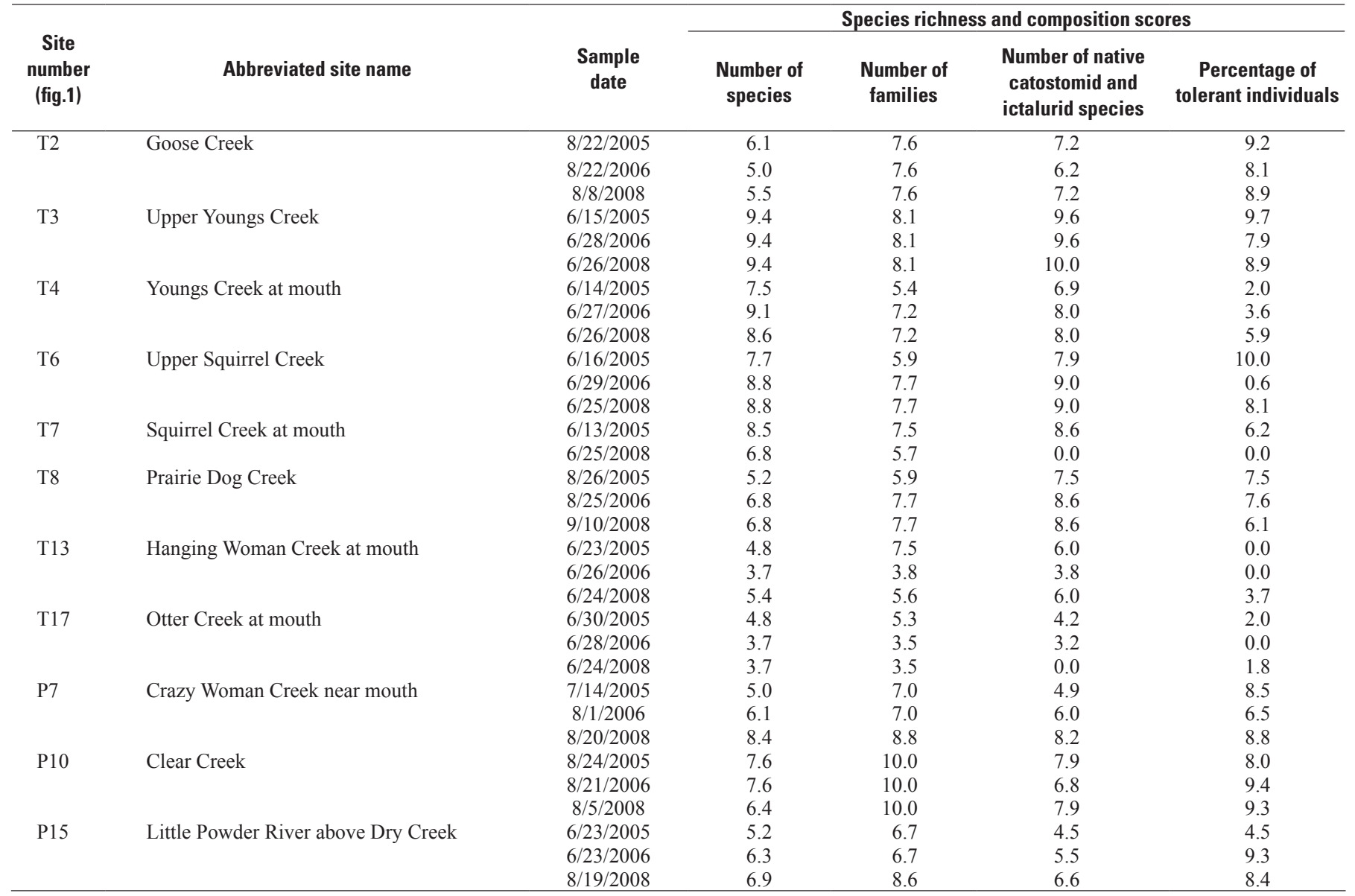

Although the number of individuals varied among years, the mean number of species collected per site was similar between sampled years: 8.8 species in 2005, 9.6 species in 2006, and 9.1 species in 2008 . The mean number of species collected per site in 2004 was 10, but only five sites were sampled in that year.

\section{Community Structure}

The Index of Biotic Integrity (IBI) developed for small prairie streams in Montana (Bramblett and others, 2005) was applied to the fish community data from the tributary streams of the Powder River by using the same metrics used for the Tongue River tributaries. Because the IBI was not intended for use in rivers with large drainage areas, raw and unadjusted fish community metric values were evaluated for the main-stem Powder River (table 18).

Results of ANOVA with the Tukey method show that there was no significant difference for fish community metrics among sites or years in the main-stem Powder River except for percentage of tolerant individuals and the percentage of native individuals (table 15). The percentage of tolerant individuals generally was higher at sites P3 and P4 than at sites farther downstream, and the percentage of native individuals generally was higher at sites P5, P8, P9, P11, and P12 than at other sites on the main-stem Powder River (fig. 17).

The fish community at Crazy Woman Creek near mouth (site P7) had the highest IBI score, 81 in 2008, among the Powder River tributaries that were sampled (table 16). IBI scores from Clear Creek (site P10) and Little Powder River above Dry Creek (site P15) also had relatively high IBI scores ranging from 62 to 70 for site $\mathrm{P} 10$ and from 59 to 72 for two of three scores for site P15. The IBI scores for the Little Powder River above Dry Creek (site P15) trended upward from 2005 to 2008 with scores ranging from 45 to 72 (fig. 18 and table 16), with a mean of 59. Crazy Woman Creek above mouth (site P7) had IBI scores ranging from 61 to 81 with a mean of 67. Clear Creek (site P10) had relatively static IBI scores from 2005 to 2008 (fig. 18). 
Table 16. Fish community metric and Index of Biotic Integrity (IBI) scores for the Tongue and Powder River tributaries, Wyoming and Montana, 2005-06 and 2008.-Continued

[Metric definitions from Bramblett and others (2005)]

\begin{tabular}{|c|c|c|c|c|c|c|c|}
\hline \multirow[b]{2}{*}{$\begin{array}{c}\text { Site } \\
\text { number } \\
\text { (fig.1) }\end{array}$} & \multicolumn{2}{|c|}{ Trophic composition scores } & \multicolumn{2}{|c|}{ Reproductive guild composition scores } & \multicolumn{3}{|c|}{ Fish abundance and composition scores } \\
\hline & $\begin{array}{l}\text { Percentage of } \\
\text { invertivorous } \\
\text { cyprinid individuals }\end{array}$ & $\begin{array}{c}\text { Number of } \\
\text { benthic } \\
\text { invertivorous } \\
\text { species }\end{array}$ & $\begin{array}{l}\text { Percentage of } \\
\text { litho-obligate } \\
\text { reproductive } \\
\text { guild"individuals }\end{array}$ & $\begin{array}{l}\text { Percentage of tolerant } \\
\text { reproducetive guild } \\
\text { individuals }\end{array}$ & $\begin{array}{c}\text { Percentage of } \\
\text { native individuals }\end{array}$ & $\begin{array}{l}\text { Number of native } \\
\text { species with } \\
\text { long-lived } \\
\text { individuals }\end{array}$ & $\begin{array}{c}\mid \mathrm{IB|} \\
\text { Score }\end{array}$ \\
\hline \multirow[t]{3}{*}{$\mathrm{T} 2$} & 0.1 & 8.2 & 2.2 & 9.0 & 1.8 & 7.6 & 59 \\
\hline & 0.2 & 6.5 & 2.7 & 8.2 & 2.2 & 7.6 & 54 \\
\hline & 0.1 & 8.2 & 1.0 & 0.4 & 0.9 & 10.0 & 50 \\
\hline \multirow[t]{3}{*}{$\mathrm{T} 3$} & 10.0 & 10.0 & 10.0 & 0.3 & 10.0 & 10.0 & 87 \\
\hline & 10.0 & 10.0 & 10.0 & 1.5 & 10.0 & 10.0 & 87 \\
\hline & 10.0 & 10.0 & 10.0 & 10.0 & 10.0 & 10.0 & 96 \\
\hline \multirow[t]{3}{*}{$\mathrm{T} 4$} & 2.8 & 10.0 & 2.5 & 8.6 & 10.0 & 9.9 & 66 \\
\hline & 1.5 & 10.0 & 1.7 & 6.3 & 10.0 & 9.9 & 67 \\
\hline & 7.5 & 10.0 & 8.6 & 8.9 & 8.1 & 9.9 & 83 \\
\hline \multirow[t]{3}{*}{ T6 } & 10.0 & 10.0 & 10.0 & 0.0 & 10.0 & 9.9 & 81 \\
\hline & 1.4 & 9.5 & 1.4 & 9.9 & 10.0 & 8.9 & 67 \\
\hline & 10.0 & 10.0 & 1.5 & 8.7 & 9.9 & 10.0 & 84 \\
\hline \multirow[t]{2}{*}{$\mathrm{T} 7$} & 8.8 & 9.0 & 7.8 & 3.3 & 9.3 & 9.5 & 78 \\
\hline & 0.0 & 0.0 & 0.0 & 4.3 & 5.0 & 9.5 & 10 \\
\hline \multirow[t]{3}{*}{ T8 } & 0.1 & 6.8 & 8.4 & 3.3 & 6.9 & 7.8 & 59 \\
\hline & 8.3 & 10.0 & 10.0 & 1.2 & 8.6 & 8.9 & 78 \\
\hline & 0.6 & 10.0 & 7.5 & 6.0 & 6.4 & 10.0 & 70 \\
\hline \multirow[t]{3}{*}{$\mathrm{T} 13$} & 0.0 & 4.6 & 0.8 & 8.9 & 8.4 & 5.3 & 46 \\
\hline & 0.0 & 4.6 & 0.0 & 10.0 & 10.0 & 4.3 & 40 \\
\hline & 0.0 & 8.0 & 0.5 & 3.6 & 6.0 & 5.3 & 44 \\
\hline \multirow[t]{3}{*}{$\mathrm{T} 17$} & 0.0 & 3.8 & 0.4 & 7.8 & 9.4 & 3.6 & 41 \\
\hline & 0.0 & 3.8 & 0.5 & 9.6 & 8.8 & 4.6 & 38 \\
\hline & 0.0 & 0.0 & 2.7 & 1.4 & 9.8 & 5.6 & 29 \\
\hline \multirow[t]{3}{*}{ P7 } & 3.2 & 5.0 & 9.9 & 0.0 & 10.0 & 7.2 & 61 \\
\hline & 1.5 & 5.0 & 10.0 & 0.4 & 9.9 & 8.2 & 61 \\
\hline & 1.1 & 6.7 & 10.0 & 8.9 & 9.9 & 10.0 & 81 \\
\hline \multirow[t]{3}{*}{ P10 } & 0.3 & 4.7 & 6.2 & 4.4 & 6.2 & 10.0 & 65 \\
\hline & 3.5 & 6.4 & 6.2 & 2.4 & 7.7 & 10.0 & 70 \\
\hline & 0.4 & 6.4 & 3.8 & 3.3 & 4.8 & 10.0 & 62 \\
\hline \multirow[t]{3}{*}{ P15 } & 0.4 & 2.8 & 6.2 & 3.7 & 8.0 & 3.6 & 45 \\
\hline & 0.5 & 4.5 & 10.0 & 0.3 & 9.5 & 6.7 & 59 \\
\hline & 0.1 & 6.2 & 9.0 & 8.5 & 9.2 & 8.7 & 72 \\
\hline
\end{tabular}

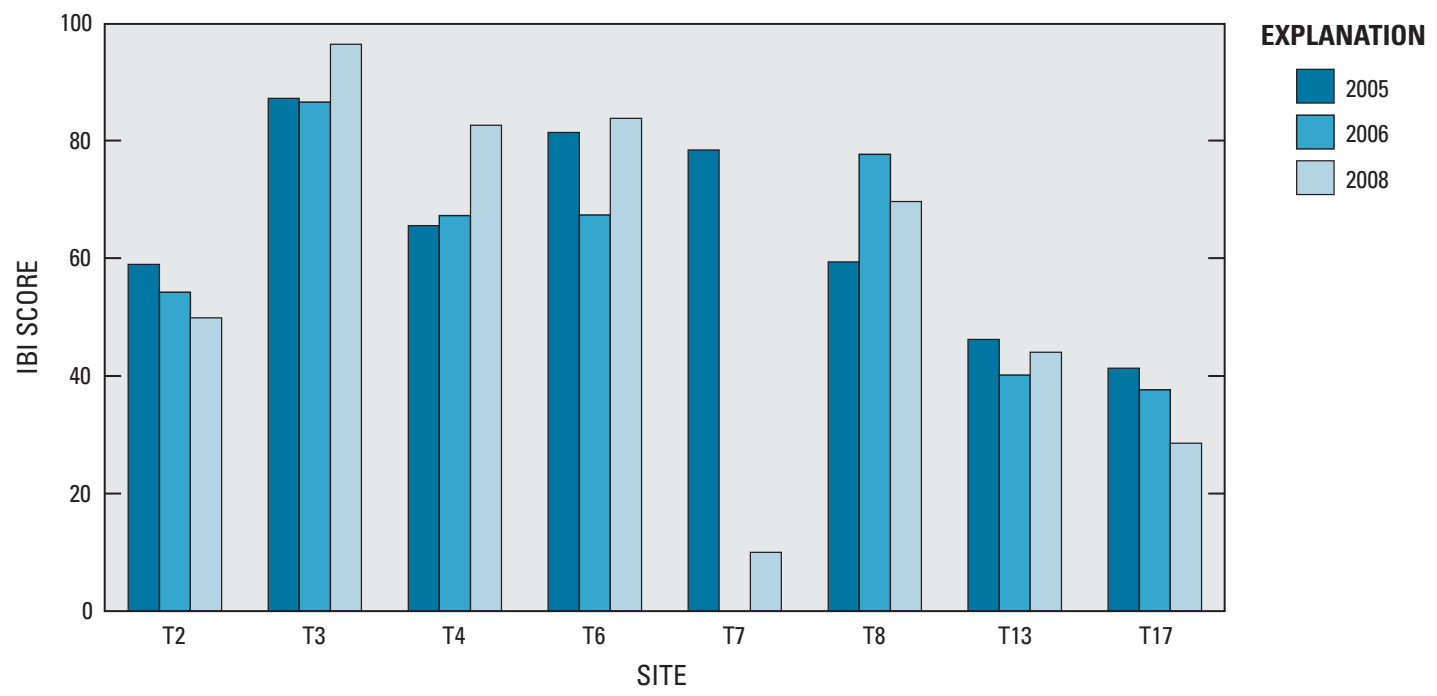

Figure 15. Index of Biotic Integrity scores (IBI) for fish communities of the Tongue River tributaries, Wyoming and Montana, 2005-06 and 2008. 
Table 17. Fish abundance in samples from Powder River drainage basin, Wyoming and Montana, 2004-06 and 2008.

[Shaded cells indicate main-stem sampling sites on the Powder River. *, Hybognathus spp. represents genus level identifications in the field; subsamples of Hybognathus spp. were retained from selected samples for laboratory identification, and are subdivided by species, either plains minnow or western silvery minnow. L, species that were only identified in larval fish samples; the total number of species includes laboratory identification of larval fish and Hybognathus spp.]

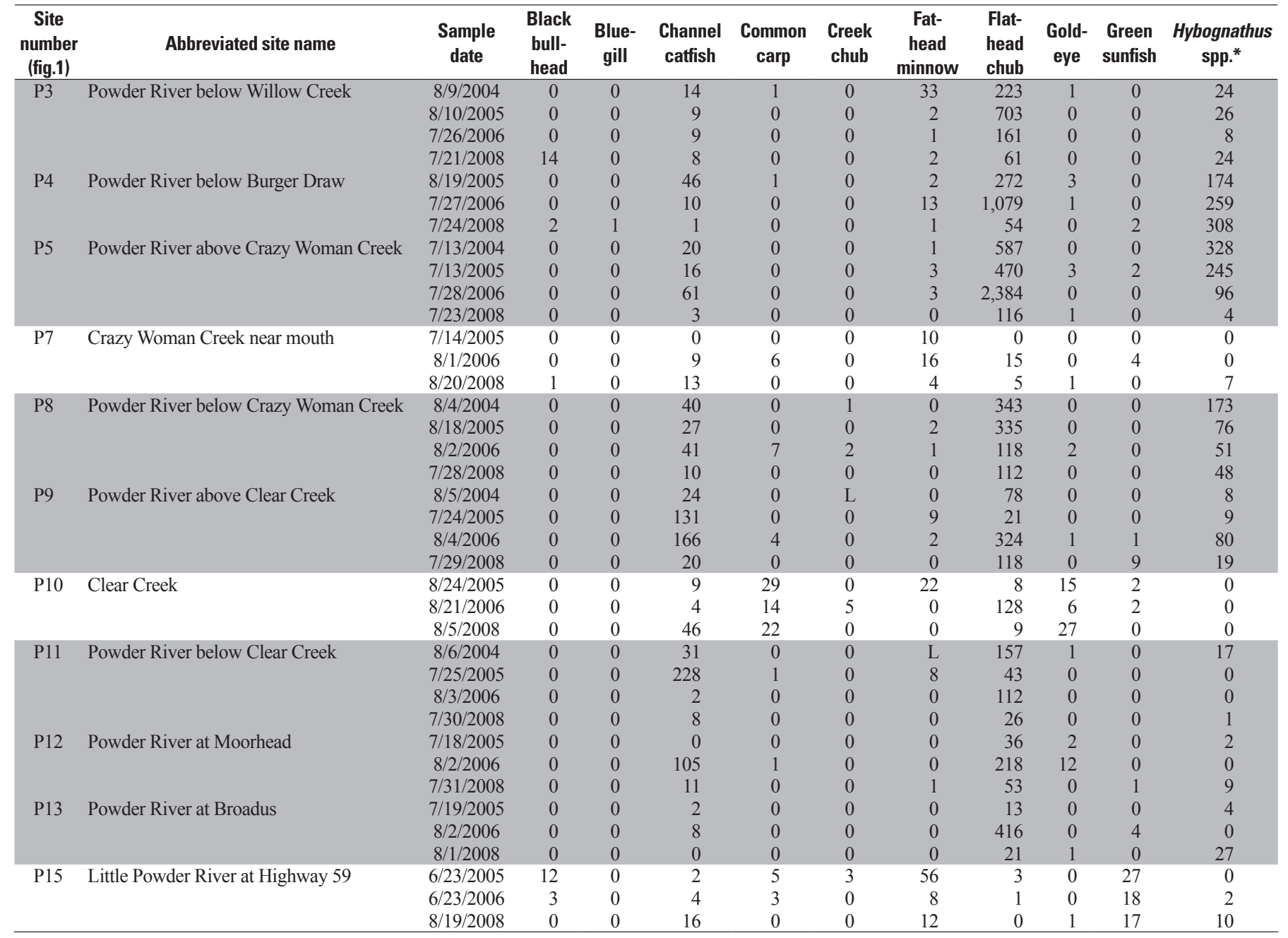

\section{Potential Effects of Water Produced from Coalbed Natural Gas Development on Biological Communities}

The ecological data presented in this report were collected to identify current conditions (2005-08) for aquatic biota and their habitat. Although no specific discharges of CBNG effluent were addressed in this study, a synthesis of the water-quality, macroinvertebrate, algal, and fish data is presented addressing existing and potential effects of CBNG produced water on biological communities.

Water production associated with CBNG development during 2005-08 tended to be largest in the Powder and Tongue River drainage basins in Wyoming. Among all of the drainages in the study area, the upper Powder River drainage basin had the largest increase between 2006 and 2008 in the amount of
CBNG water production (fig. 2). For example, water production for the upper Powder River drainage basin in Wyoming during 2008 was more than twice as much compared to production prior to that time. It should be noted, however, that not all of the production water reaches the streams because of various methods of treatment and disposal.

\section{Water Quality}

Most water produced during CBNG development in Wyoming is discharged into constructed reservoirs or surface drainages where the water may infiltrate, become part of the ambient streamflow, or evaporate. Concerns have been expressed regarding the quality of some waters produced during CBNG development and effects produced waters may have on water quality in streams that drain the CBNGdevelopment area. Sodium and bicarbonate are the dominant 
Table 17. Fish abundance in samples from Powder River drainage basin, Wyoming and Montana, 2004-06 and 2008.—Continued

[Shaded cells indicate main-stem sampling sites on the Powder River. *, Hybognathus spp. represents genus level identifications in the field; subsamples of Hybognathus spp. were retained from selected samples for laboratory identification, and are subdivided by species, either plains minnow or western silvery minnow. L, species that were only identified in larval fish samples; the total number of species includes laboratory identification of larval fish and Hybognathus spp.]

\begin{tabular}{|c|c|c|c|c|c|c|c|c|c|c|c|c|c|c|c|c|}
\hline $\begin{array}{c}\text { Site } \\
\text { number } \\
\text { (fig.1) }\end{array}$ & $\begin{array}{c}\text { Plains } \\
\text { minnow* }\end{array}$ & $\begin{array}{c}\text { Western } \\
\text { silvery } \\
\text { minnow* }\end{array}$ & $\begin{array}{l}\text { Long- } \\
\text { nose } \\
\text { dace }\end{array}$ & $\begin{array}{c}\text { Moun- } \\
\text { tain } \\
\text { sucker }\end{array}$ & $\begin{array}{c}\text { Northern } \\
\text { plains } \\
\text { killifish }\end{array}$ & $\begin{array}{c}\text { River } \\
\text { carp- } \\
\text { sucker }\end{array}$ & $\begin{array}{l}\text { Rock } \\
\text { bass }\end{array}$ & $\begin{array}{l}\text { Sand } \\
\text { shiner }\end{array}$ & Sauger & $\begin{array}{c}\text { Short- } \\
\text { head } \\
\text { redhorse }\end{array}$ & $\begin{array}{c}\text { Shovel- } \\
\text { nose } \\
\text { sturgeon }\end{array}$ & $\begin{array}{c}\text { Small- } \\
\text { mouth } \\
\text { bass }\end{array}$ & $\begin{array}{c}\text { Stone- } \\
\text { cat }\end{array}$ & $\begin{array}{l}\text { Stur- } \\
\text { geon } \\
\text { chub }\end{array}$ & $\begin{array}{l}\text { White } \\
\text { sucker }\end{array}$ & $\begin{array}{c}\text { Total } \\
\text { number } \\
\text { of species }\end{array}$ \\
\hline \multirow[t]{4}{*}{ P3 } & 24 & 0 & 28 & 1 & 27 & 8 & 0 & 487 & 0 & 0 & 0 & 0 & 1 & 0 & 0 & 12 \\
\hline & 26 & 0 & 17 & 0 & 80 & 70 & 0 & 847 & 0 & 0 & 0 & 0 & 1 & 0 & 1 & 10 \\
\hline & 0 & 0 & 24 & 0 & 164 & 50 & 0 & 4,486 & 0 & 0 & 0 & 0 & 4 & 0 & 0 & 9 \\
\hline & 24 & 0 & 0 & 0 & 5 & 1 & 0 & 51 & 0 & 0 & 0 & 0 & 0 & 0 & 0 & 8 \\
\hline \multirow[t]{3}{*}{ P4 } & 166 & 8 & 52 & 0 & 197 & 68 & 0 & 685 & 0 & 0 & 0 & 0 & 0 & 0 & 0 & 11 \\
\hline & 0 & 0 & 70 & 0 & 43 & 46 & 0 & 3,789 & 0 & 0 & 0 & 0 & 2 & 0 & 0 & 10 \\
\hline & 188 & 120 & 18 & 0 & 8 & 5 & 0 & 123 & 0 & 0 & 0 & 0 & 3 & 0 & 0 & 13 \\
\hline \multirow[t]{4}{*}{ P5 } & 319 & 9 & 15 & 0 & 8 & 6 & 0 & 1,294 & 0 & 0 & 0 & 0 & 1 & 0 & 0 & 10 \\
\hline & 245 & 0 & 2 & 0 & 4 & 136 & 0 & 1,668 & 0 & 1 & 0 & 0 & 0 & 0 & 0 & 11 \\
\hline & 0 & 0 & 17 & 0 & 20 & 75 & 0 & 3,104 & 0 & 0 & 0 & 0 & 0 & 2 & 0 & 9 \\
\hline & 4 & 0 & 0 & 0 & 1 & 21 & 0 & 68 & 0 & 0 & 0 & 0 & 0 & 0 & 0 & 7 \\
\hline \multirow[t]{3}{*}{ P7 } & 0 & 0 & 3 & 0 & 0 & 0 & 0 & 33 & 0 & 0 & 0 & 0 & 1 & 0 & 8 & 5 \\
\hline & 0 & 0 & 67 & 0 & 0 & 0 & 0 & 402 & 0 & 0 & 0 & 0 & 9 & 0 & 227 & 9 \\
\hline & 0 & 7 & 11 & 0 & 0 & 0 & 0 & 129 & 0 & 3 & 0 & 1 & 1 & 0 & 16 & 13 \\
\hline \multirow[t]{4}{*}{ P8 } & 173 & 0 & 33 & 0 & 0 & 2 & 0 & 933 & 0 & 1 & 0 & 0 & 12 & 0 & 0 & 9 \\
\hline & 0 & 0 & 29 & 0 & 15 & 4 & 0 & 875 & 0 & 0 & 0 & 0 & 0 & 0 & 0 & 8 \\
\hline & 0 & 0 & 14 & 0 & 3 & 182 & 0 & 1,903 & 0 & 3 & 0 & 0 & 0 & 0 & 0 & 12 \\
\hline & 48 & 0 & 0 & 0 & 1 & 9 & 0 & 160 & 0 & 0 & 0 & 0 & 0 & 0 & 0 & 6 \\
\hline \multirow[t]{4}{*}{ P9 } & 8 & 0 & 1 & 0 & 1 & 2 & 0 & 446 & 0 & 0 & 0 & 0 & 2 & 0 & 0 & 9 \\
\hline & 0 & 0 & 0 & 0 & 0 & 4 & 0 & 370 & 0 & 0 & 0 & 0 & 0 & 0 & 0 & 6 \\
\hline & 0 & 0 & 0 & 0 & 0 & 25 & 0 & 794 & 0 & 0 & 0 & 0 & 0 & 0 & 0 & 9 \\
\hline & 10 & 9 & 0 & 0 & 1 & 4 & 0 & 192 & 0 & 0 & 0 & 0 & 2 & 0 & 0 & 9 \\
\hline \multirow[t]{3}{*}{ P10 } & 0 & 0 & 0 & 0 & 0 & 13 & 91 & 71 & 2 & 40 & 0 & 22 & 32 & 0 & 19 & 14 \\
\hline & 0 & 0 & 1 & 0 & 0 & 0 & 76 & 192 & 1 & 12 & 0 & 33 & 45 & 0 & 13 & 14 \\
\hline & 0 & 0 & 0 & 0 & 0 & 29 & 96 & 0 & 2 & 25 & 0 & 56 & 14 & 0 & 0 & 11 \\
\hline \multirow[t]{4}{*}{ P11 } & 17 & 0 & 2 & 0 & 0 & 3 & 0 & 687 & 0 & 0 & 0 & 1 & 7 & 0 & 0 & 10 \\
\hline & 0 & 0 & 0 & 0 & 0 & 25 & 0 & 404 & 0 & 0 & 0 & 1 & 17 & 0 & $\mathrm{~L}$ & 10 \\
\hline & 0 & 0 & 4 & 0 & 0 & 3 & 0 & 289 & 0 & 0 & 0 & 0 & 3 & 0 & 0 & 6 \\
\hline & 1 & 0 & 0 & 0 & 0 & 0 & 0 & 18 & 0 & 0 & 0 & 0 & 1 & 0 & 0 & 5 \\
\hline \multirow[t]{3}{*}{ P12 } & 1 & 1 & 11 & 0 & 0 & 0 & 0 & 14 & 0 & 0 & 0 & 0 & 0 & 0 & 0 & 6 \\
\hline & 0 & 0 & 6 & 0 & 0 & 116 & 0 & 283 & 0 & 2 & 0 & 0 & 1 & 4 & 0 & 10 \\
\hline & 9 & 0 & 2 & 0 & 0 & 1 & 0 & 9 & 0 & 0 & 0 & 0 & 12 & 1 & 0 & 10 \\
\hline \multirow[t]{3}{*}{ P13 } & 4 & 0 & 246 & 0 & 6 & 0 & 0 & 39 & 0 & 0 & 0 & 0 & 0 & 1 & 0 & 7 \\
\hline & 0 & 0 & 55 & 0 & 28 & 43 & 0 & 559 & 0 & 0 & 0 & 0 & 0 & 0 & 0 & 7 \\
\hline & 19 & 8 & 16 & 0 & 0 & 1 & 0 & 64 & 0 & 0 & 1 & 0 & 0 & 0 & 0 & 8 \\
\hline \multirow[t]{3}{*}{ P15 } & 0 & 0 & 0 & 0 & 0 & 0 & 0 & 95 & 0 & 0 & 0 & 0 & 0 & 0 & 13 & 9 \\
\hline & 2 & 0 & 19 & 0 & 0 & 5 & 0 & 453 & 0 & 0 & 0 & 0 & 0 & 0 & 4 & 11 \\
\hline & 0 & 10 & 1 & 0 & 0 & 1 & 0 & 150 & 0 & 4 & 0 & 0 & 0 & 0 & 3 & 10 \\
\hline
\end{tabular}

ions in coalbed waters (Rice and others, 2000) in contrast to area stream waters, which typically have a larger ambient percentage of calcium, magnesium, and sulfate (Clark and Mason, 2007). The primary constituents of concern in CBNG produced water are sodium, sodium-adsorption ratio, specific conductance, and dissolved solids because large values for these constituents have the potential to negatively affect the water for irrigation uses (Brown and Johnston, 2005).

The potential effects of CBNG discharges on biological communities include several aspects of water quality. Increases in sodium bicarbonate concentrations in streams to potentially toxic levels as a result of CBNG produced water inputs are of concern to aquatic life (Bureau of Land Management, 2003). Larval fish that were exposed to sodium bicarbonate had lower hatch and survival rates compared to controls for both acute and chronic exposures (Skaar and others, 2006). Coalbed waters, which have a relatively constant temperature compared to streams, have the potential to increase or decrease stream temperature depending on the time of year. Turbidity associated with coalbed waters may be lower than the natural stream turbidity; however, increased concentrations of suspended sediment resulting from indirect CBNG-development activities such as road construction and channel erosion may increase stream turbidity. Increased sedimentation also has potential to affect substrate and spawning habitat. Coalbed waters typically are anoxic and reducing, which increases the solubility of some trace elements, including iron, manganese, and barium (Rice and others, 2000). The discharge of anoxic coalbed waters to streams could affect dissolved-oxygen concentrations of streams and affect the precipitation of selected trace elements, particularly iron and manganese. Data collected from ATG sites indicated that iron and manganese concentrations were higher than the chronic and, in a few cases, the acute aquatic-life criteria. The mobilization of other trace elements, including arsenic and selenium, in basin sediments from the addition of coalbed waters to the 


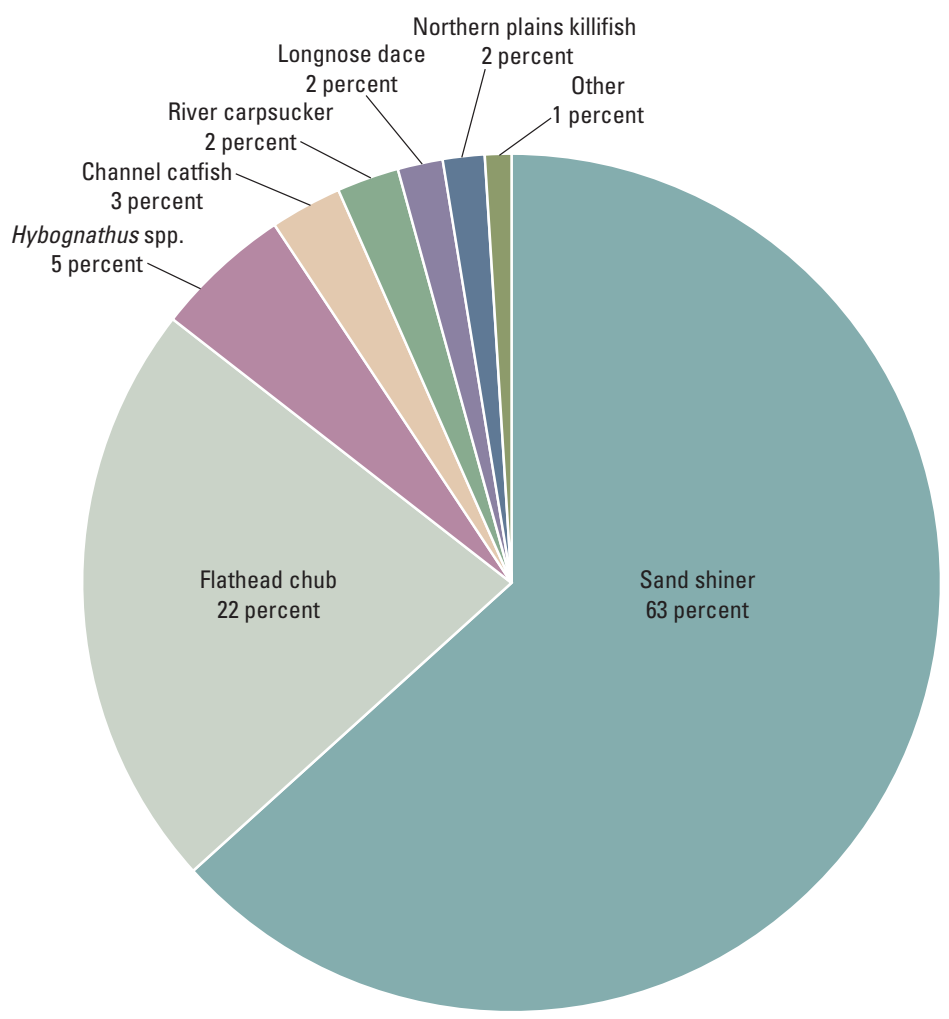

Main-stem Powder River

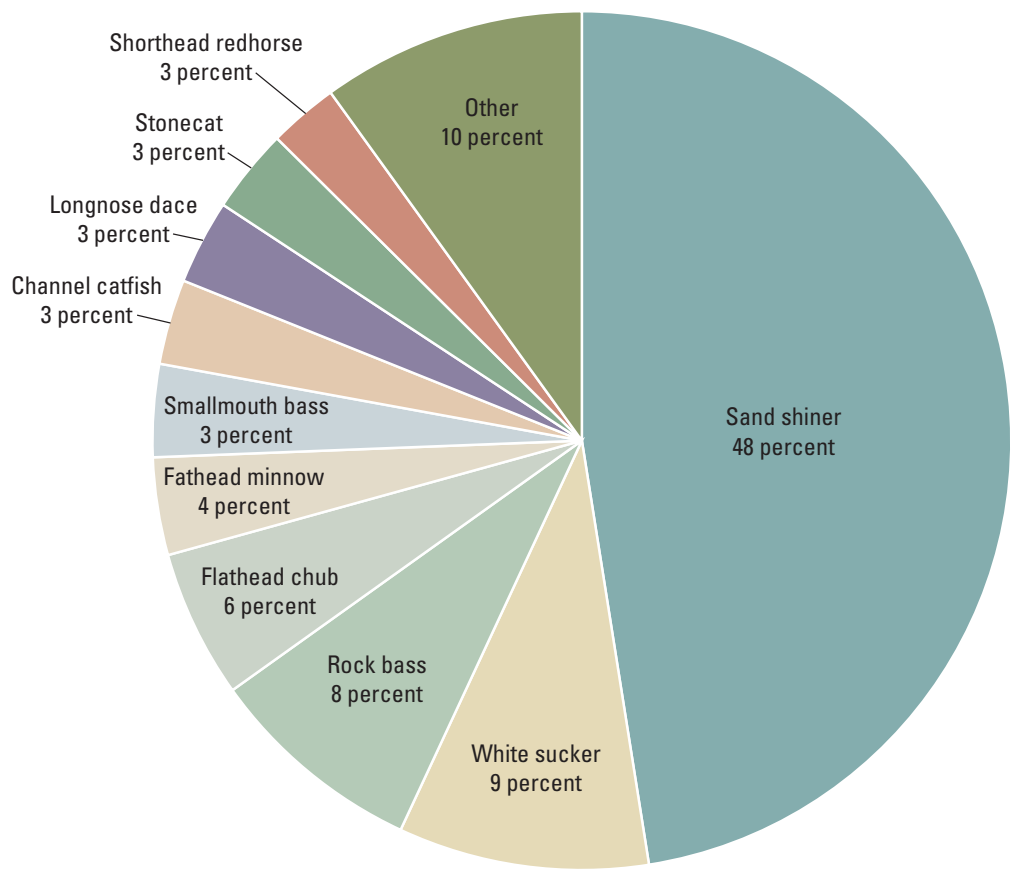

Powder River tributaries

Figure 16. Relative abundance of fish by species in samples from the Powder River drainage basin, Wyoming and Montana, 2004-06 and 2008. 
Table 18. Unadjusted fish community metric values for the main-stem Powder River, Wyoming and Montana, 2004-06 and 2008.

[Metric definitions from Bramblett and others (2005)]

\begin{tabular}{|c|c|c|c|c|c|c|c|c|c|c|c|c|}
\hline \multirow[b]{2}{*}{$\begin{array}{c}\text { Site } \\
\text { number } \\
\text { (fig. 1) }\end{array}$} & \multirow[b]{2}{*}{ Abbreviated site name } & \multirow[b]{2}{*}{$\begin{array}{l}\text { Sample } \\
\text { date }\end{array}$} & \multicolumn{5}{|c|}{ Number of } & \multicolumn{5}{|c|}{ Percentage of } \\
\hline & & & $\begin{array}{l}\text { Native } \\
\text { species }\end{array}$ & $\begin{array}{c}\text { Native } \\
\text { families }\end{array}$ & $\begin{array}{c}\text { Native } \\
\text { catostomid } \\
\text { and ictalurid } \\
\text { species }\end{array}$ & $\begin{array}{c}\text { Bbenthic } \\
\text { invertivorous } \\
\text { species }\end{array}$ & $\begin{array}{l}\text { Native } \\
\text { long-lived } \\
\text { species }\end{array}$ & $\begin{array}{c}\text { Tolerant } \\
\text { individuals }\end{array}$ & $\begin{array}{l}\text { Invertivorous } \\
\text { cyprinid } \\
\text { individuals }\end{array}$ & $\begin{array}{l}\text { Litho-obligate } \\
\text { reproductive } \\
\text { guild individuals }\end{array}$ & $\begin{array}{c}\text { Tolerant } \\
\text { reproductive } \\
\text { guild } \\
\text { individuals }\end{array}$ & $\begin{array}{c}\text { Native } \\
\text { individuals }\end{array}$ \\
\hline \multirow[t]{4}{*}{ P3 } & Powder River below Willow Creek & $8 / 9 / 2004$ & 11 & 4 & 4 & 1 & 4 & 7.2 & 29.6 & 61.9 & 5.5 & 96.7 \\
\hline & & $8 / 10 / 2005$ & 9 & 3 & 4 & 1 & 4 & 4.7 & 41.0 & 53.3 & 0.6 & 95.4 \\
\hline & & $7 / 26 / 2006$ & 7 & 3 & 3 & 1 & 5 & 3.4 & 3.8 & 93.0 & 0.2 & 96.7 \\
\hline & & $7 / 21 / 2008$ & 6 & 3 & 2 & 0 & 3 & 12.7 & 36.7 & 31.3 & 14.5 & 88.6 \\
\hline \multirow[t]{3}{*}{ P4 } & Powder River below Burger Draw & $8 / 19 / 2005$ & 10 & 4 & 2 & 1 & 6 & 13.3 & 21.6 & 53.9 & 3.2 & 86.8 \\
\hline & & $7 / 27 / 2006$ & 8 & 4 & 3 & 1 & 7 & 1.1 & 21.6 & 73.6 & 0.4 & 99.2 \\
\hline & & $7 / 24 / 2008$ & 9 & 3 & 3 & 1 & 5 & 3.2 & 13.6 & 28.3 & 1.5 & 97.5 \\
\hline \multirow[t]{4}{*}{ P5 } & Powder River above Crazy Woman Creek & $7 / 13 / 2004$ & 9 & 3 & 3 & 1 & 3 & 0.4 & 26.6 & 58.2 & 0.9 & 99.6 \\
\hline & & $7 / 13 / 2005$ & 9 & 4 & 3 & 2 & 5 & 0.4 & 18.5 & 71.0 & 0.7 & 99.8 \\
\hline & & $7 / 28 / 2006$ & 7 & 3 & 2 & 2 & 5 & 0.4 & 41.7 & 55.5 & 1.1 & 99.7 \\
\hline & & $7 / 23 / 2008$ & 6 & 4 & 2 & 0 & 1 & 0.5 & 54.2 & 42.1 & 1.4 & 99.5 \\
\hline \multirow[t]{4}{*}{ P8 } & Powder River below Crazy Woman Creek & $8 / 4 / 2004$ & 9 & 3 & 4 & 2 & 6 & 0.0 & 24.5 & 63.8 & 2.6 & 100.0 \\
\hline & & $8 / 18 / 2005$ & 7 & 3 & 2 & 1 & 4 & 1.2 & 26.7 & 66.6 & 2.1 & 98.9 \\
\hline & & $8 / 2 / 2006$ & 10 & 4 & 3 & 2 & 5 & 0.5 & 5.8 & 90.5 & 1.8 & 99.6 \\
\hline & & $7 / 28 / 2008$ & 5 & 3 & 2 & 0 & 1 & 0.3 & 32.9 & 49.7 & 2.9 & 99.7 \\
\hline \multirow[t]{4}{*}{ P9 } & Powder River above Clear Creek & $8 / 5 / 2004$ & 8 & 3 & 3 & 1 & 5 & 0.2 & 14.1 & 80.2 & 4.3 & 99.8 \\
\hline & & $7 / 24 / 2005$ & 6 & 3 & 2 & 0 & 2 & 1.7 & 3.9 & 68.8 & 25.7 & 100.0 \\
\hline & & $8 / 4 / 2006$ & 7 & 4 & 2 & 0 & 6 & 0.5 & 23.2 & 58.7 & 12.0 & 99.6 \\
\hline & & $7 / 29 / 2008$ & 7 & 3 & 3 & 0 & 3 & 2.7 & 32.3 & 54.2 & 5.5 & 97.3 \\
\hline \multirow[t]{4}{*}{ P11 } & Powder River below Clear Creek & $8 / 6 / 2004$ & 9 & 4 & 3 & 1 & 6 & 0.0 & 17.5 & 77.3 & 3.5 & 99.9 \\
\hline & & $7 / 25 / 2005$ & 9 & 3 & 4 & 0 & 5 & 1.2 & 5.9 & 61.3 & 32.6 & 99.7 \\
\hline & & $8 / 3 / 2006$ & 6 & 3 & 3 & 1 & 2 & 0.0 & 28.1 & 72.4 & 0.5 & 100.0 \\
\hline & & $7 / 30 / 2008$ & 5 & 2 & 2 & 0 & 0 & 0 & 48.1 & 35.2 & 14.8 & 100.0 \\
\hline \multirow[t]{3}{*}{$\mathrm{P} 12$} & Powder River at Moorhead & $7 / 18 / 2005$ & 6 & 2 & 0 & 1 & 1 & 0.0 & 72.3 & 41.5 & 0.0 & 100.0 \\
\hline & & $8 / 2 / 2006$ & 10 & 4 & 4 & 3 & 7 & 0.1 & 30.5 & 56.7 & 14.0 & 99.9 \\
\hline & & $7 / 31 / 2008$ & 9 & 3 & 3 & 2 & 5 & 2 & 56.0 & 25.0 & 12.0 & 99.0 \\
\hline \multirow[t]{3}{*}{$\mathrm{P} 13$} & Powder River at Broadus & $7 / 19 / 2005$ & 6 & 2 & 1 & 2 & 2 & 1.9 & 83.6 & 92.0 & 0.6 & 98.1 \\
\hline & & $8 / 2 / 2006$ & 5 & 3 & 2 & 1 & 1 & 2.9 & 42.3 & 59.0 & 0.7 & 97.1 \\
\hline & & $8 / 1 / 2008$ & 8 & 4 & 1 & 2 & 6 & 0 & 28.2 & 63.4 & 0.0 & 100.0 \\
\hline
\end{tabular}



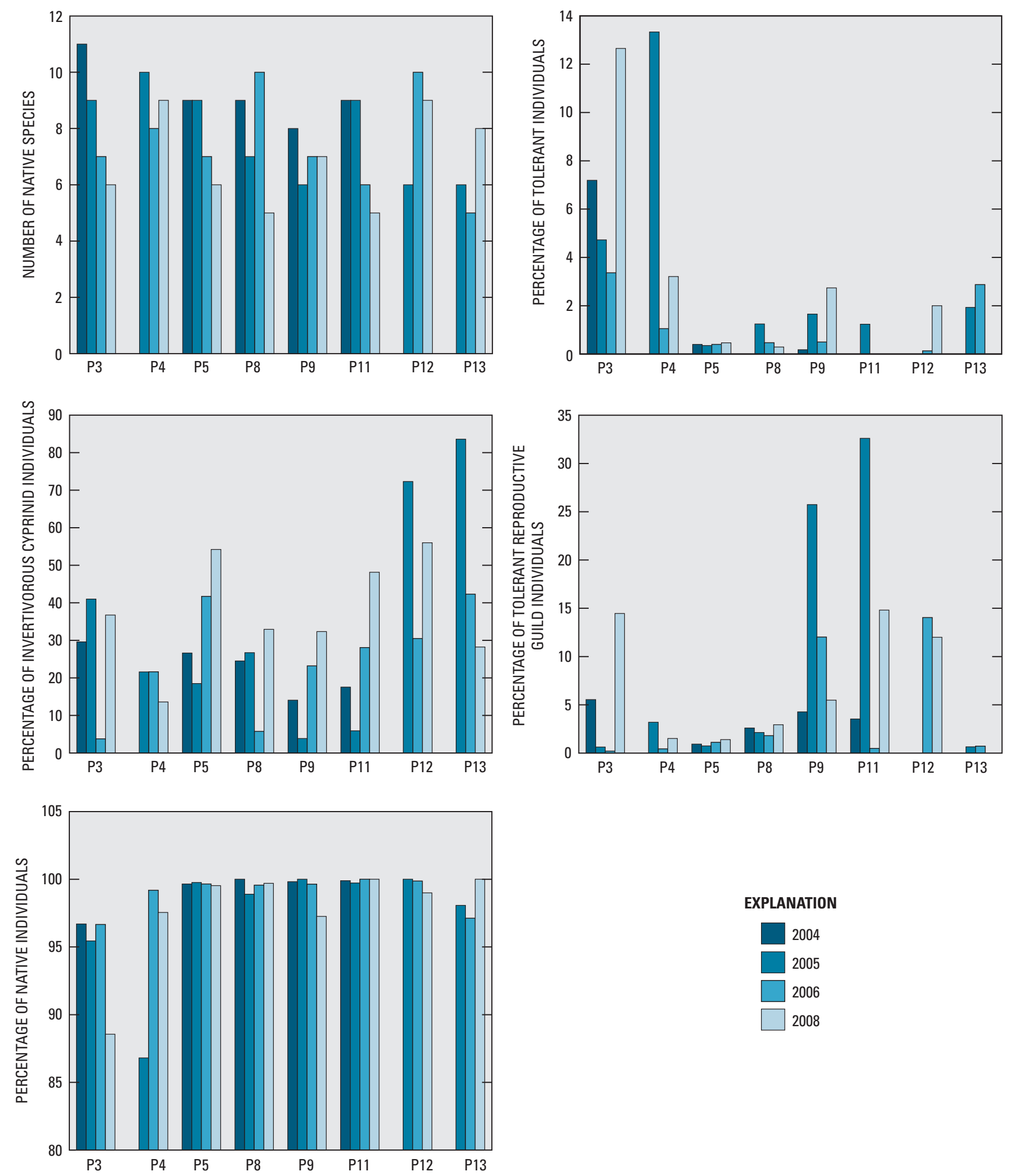

Figure 17. Selected unadjusted fish community metric values for the main-stem Powder River, Wyoming and Montana, 2004-06 and 2008. 


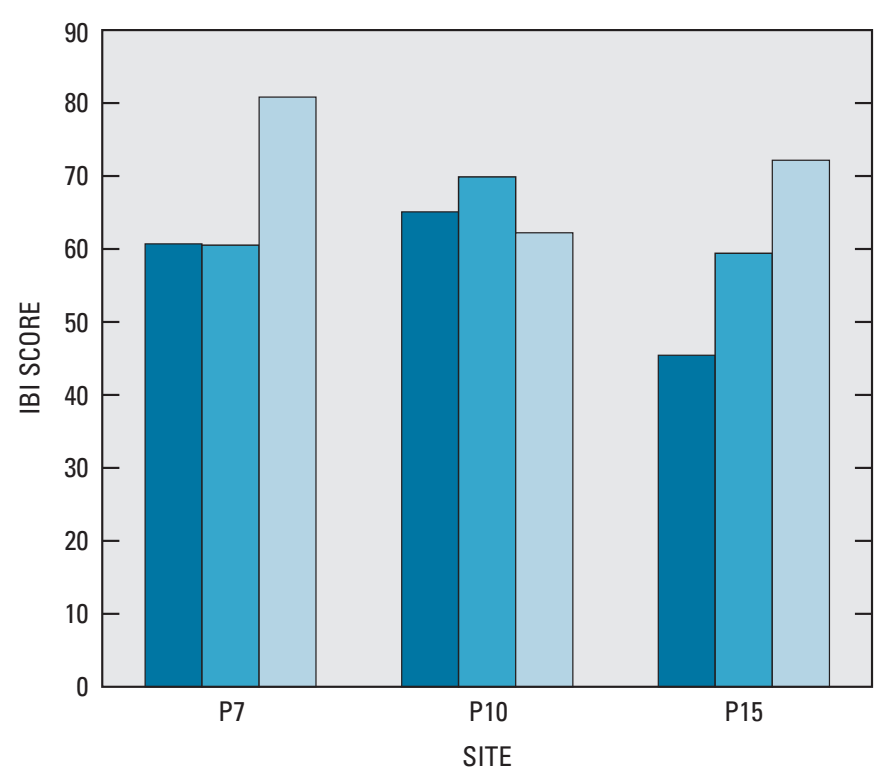

EXPLANATION

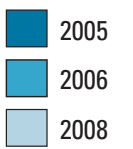

Figure 18. Index of Biotic Integrity (IBI) scores for fish communities in selected Powder River tributaries, Wyoming, 2005-06 and 2008.

surface also has been identified as a potential water-quality concern (Brinck and others, 2008). Nutrient cycling in streams also may be affected by inputs of coalbed waters, which can contain elevated concentrations of ammonia. Increases in inputs of inorganic nitrogen could increase the potential for eutrophication (Smith and others, 2009).

\section{Macroinvertebrate Communities}

Macroinvertebrate communities of the main-stem Tongue River indicated some differences in community composition in the downstream direction. For example, the relative abundance of noninsects at a site on the main-stem Tongue River downstream from Tongue River Reservoir during 2005-08 was significantly different from other sites on the main stem upstream and downstream from the reservoir. Given the differences in water quality from upstream to downstream from the reservoir, the differences in macroinvertebrate communities might be attributable to the effects of the reservoir rather than the effects of CBNG development.

Macroinvertebrate community composition in the middle reach of the main-stem Powder River, from below the confluence with Willow Creek to below the Crazy Woman Creek confluence, differed from communities in the upper and lower reaches of the Powder River. Macroinvertebrate community metric values at sampling sites in the middle reach during 2005-08 were significantly different (ANOVA, $p<0.05$ ) from metric values at other sites on the main-stem Powder River.
The affected metrics included taxa richness, relative abundance, feeding modes, and tolerance, which were indicative of communitywide differences. Ephemeroptera taxa richness, Ephemeroptera relative abundance, and intolerant macroinvertebrate relative abundance tended to be lower, whereas Diptera relative abundance and filterer-collector relative abundance tended to be higher, in the middle reach of the Powder River than in other reaches.

Potential causes of the observed pattern in macroinvertebrate communities of the main-stem Powder River include natural influences, coalbed natural gas development, and other development such as conventional oil and gas development in the Salt Creek drainage. For example, chloride concentrations in the Powder River downstream from Salt Creek sometimes exceeded USEPA acute and chronic criteria for the protection of aquatic life but not WDEQ site-specific criteria. Testing of relations between environmental variables and the macroinvertebrate taxonomy and metrics from the Powder River indicated some of the variation in the macroinvertebrate communities could be explained by variation in the environmental variables. The environmental variables that best correlated with the macroinvertebrates communities included physical variables (turbidity, embeddedness, $\mathrm{D}_{84}$, and streamflow) and chemical variables (alkalinity and specific conductance). The Powder River is known for its relatively high transport of fine sediment (Lowry and others, 1986), which in turn can affect turbidity, embeddedness, and $\mathrm{D}_{84}$. Of the environmental variables correlated with the macroinvertebrate communities, alkalinity perhaps is the best indicator of potential CBNG produced water influences because of the sodium-bicarbonate signature of CBNG water (Rice and others, 2000). Mean alkalinity concentrations at the time of ecological sampling were about $200 \mathrm{mg} / \mathrm{L}$ throughout the main-stem Powder River from upstream from Salt Creek to Locate, near the mouth at the Yellowstone River, with the exception of site P4, which is downstream from Burger Draw (fig. 4). The alkalinity concentrations in the Powder River below Burger Draw (site P4) ranged from $224 \mathrm{mg} / \mathrm{L}$ in 2008 to $1,540 \mathrm{mg} / \mathrm{L}$ during the drought year of 2006, with a mean near $600 \mathrm{mg} / \mathrm{L}$. This site receives CBNG discharges from several upstream drainages.

Given that macroinvertebrate communities integrate responses to environmental variables over a longer time period than instantaneous samples, it is likely that the macroinvertebrate communities in the middle reach of the Powder River from Willow Creek to below the Crazy Woman Creek confluence are responding to variation in the measured environmental variables beyond those observed at the time of sampling or to other environmental variables that were not measured. Water production from CBNG development in the Powder River drainage more than doubled from 2006 to 2008. Discharge of CBNG produced water is a logical explanation for the observed patten in macroinvertebrate communities of the main-stem Powder River, but further study would be needed for confirmation as described in the section "Further Study Needs." 


\section{Algal Communities}

Similar to the pattern observed for the macroinvertebrates, the algal communities of the main-stem Powder River in 2005 and 2007 indicated differences in communities of the middle reach from those in the upper or lower reaches. Algal communities in the middle reach often were characterized by dominance by a single taxon and by low biovolume of algae compared to the upper and lower reaches of the Powder River.

\section{Fish Communities}

Fish species richness was relatively high in the middle reach of the main-stem Powder River, the same reach where macroinvertebrate and algal communities appeared to be anomalous. Although a few significant differences in fish metrics along the main-stem Powder River were noted, the differences did not correspond to the pattern observed for the macroinvertebrate and algae communities.

The Powder River fish assemblage has been described as largely intact as a result of the relatively unperturbed state of the river (Hubert, 1993). More recently, Senecal (2009) derived a reference fish assemblage for the Powder River on the basis of 1964-80 sampling data and compared the reference fish assemblage dataset to more current sampling data from 1994 and 2008. The reference fish assemblage using the combined 1964-80 dataset was composed predominantly of flathead chub, followed by Hybognathus spp. and sturgeon chub (Senecal, 2009). In contrast, the 2008 fish assemblage was dominated by sand shiners, Hybognathus spp., flathead chub, river carpsucker, channel catfish, and longnose dace (Senecal, 2009). The differences between the reference fish assemblage and the more recent fish assemblages were due to decreases in proportions of previously common species, such as sturgeon chub, and increases in proportions of two species, sand shiners and northern plains killifish. Senecal (2009) states that care must be taken with the results because the datasets used in the reference fish assemblage were collected at different times by different investigators, and lack of standardization across datasets may have contributed to discrepancies. However, results presented in this report are similar to those as presented by Senecal (2009). Slight differences in the relative abundance in the 2005-06 and 2008 samples, in order of dominance, were sand shiner, flathead chub, Hybognathus spp., channel catfish, river carpsucker, longnose dace, and northern plains killifish. Reasons for the increase in abundance of sand shiners are unclear.

A study conducted by Davis (2008) indicated mixed results of the effects of CBNG development on fish assemblages in the Powder River Basin during 2005-06. For example, species richness and IBI scores were similar between developed and undeveloped sites, and no strong relations existed between overall IBI scores or most IBI metric scores and the number or density of CBNG wells in a drainage area. Other evidence, however, indicated that CBNG-produced water may negatively affect fish assemblages. Conductivity was, on average, higher in streams with CBNG-produced water and was negatively related to biotic integrity. Bicarbonate, one of the primary salts in product water, appeared to be harmful to some species of fish. Altered flow regimes may present the biggest direct effect from CBNG effluent on fish assemblages (Davis, 2008; Senecal, 2009), and potential effects of CBNG development may be more apparent during wet years when more sensitive fish assemblages are present. Also, toxicity testing (Skaar and others, 2006) indicates that larval fish are more sensitive to CBNG effects than the adult and juvenile fish that were collected during this study.

\section{Further Study Needs}

The anomalous patterns of macroinvertebrate and algal communities in the middle reach of the main-stem Powder River are worthy of further study to help assess the natural or anthropogenic factors affecting the anomalous patterns. One approach to further study would be establishment of paired sampling sites upstream and downstream from known CBNG discharge points. As an aid to selecting potential sampling sites, water-quality and streamflow data from the current USGS monitoring network (http://wy.water.usgs.gov/) were evaluated. Fifteen tributaries to the Powder River in Wyoming are currently (2010) sampled in cooperation with the WDEQ. Instantaneous streamflow values indicate that other than the perennial tributaries (Salt Creek, Crazy Woman Creek, and Clear Creek) bracketed during this study, Pumpkin Creek (in Johnson County), Beaver Creek, and Wild Horse Creek (fig. 1) contribute the largest amount of streamflow to the Powder River. Although streamflow in Pumpkin, Beaver, and Wildhorse Creeks often was small compared to streamflow in the Powder River, these streams are of interest for biological monitoring because specific-conductance values and alkalinity concentrations measured in the tributaries commonly were higher than those measured in the Powder River. Permit data on file with the WDEQ indicate other tributaries, such as Indian Creek and Flying E Creek, also contribute treated and untreated CBNG discharges to the Powder River. One or more future biological monitoring sites could be located upstream from Willow Creek to help determine a reference condition upstream from CBNG discharges. Consideration to defining the spatial extent of the effects of tributaries and whether the effects are cumulative with distance downstream also may be warranted.

Additional analysis of data from the water-quality monitoring network in the Powder River and Tongue River drainage basins would be useful in determining long-term trends in water quality. Specific conductance in the Tongue River increased from Monarch (site T1) to the State line (site T9; fig. 4), but it is not known if the increase is due to effects of CBNG discharges or other influences such as irrigation return flows and discharge of treated sewage effluent. Long-term ecological monitoring also may be considered to identify trends and natural variability in biological communities in the Tongue and Powder River Basins. 


\section{Summary}

In response to concerns about the effects of coalbed natural gas development (CBNG) on streams of the Powder River structural basin in Wyoming and Montana, an interagency Aquatic Task Group (ATG) was formed in 2004. Participants in the ATG included the Bureau of Land Management (BLM), the Montana Department of Environmental Quality (MDEQ), Montana Fish, Wildlife, and Parks, the U.S. Environmental Protection Agency (USEPA), the Wyoming Department of Environmental Quality (WDEQ), the Wyoming Game and Fish Department, and the U.S. Geological Survey (USGS). The ATG developed a monitoring plan to (1) establish current ecological conditions for aquatic biota and their habitat and (2) determine existing and potential effects of CBNG-produced water on aquatic life. The sample collection performed by the USGS during 2005-08 and described in this report was conducted under the direction of the ATG to meet objective 1 of the monitoring plan and, to the extent possible, objective 2 .

Samples for environmental variables (water quality and habitat) and biological communities (macroinvertebrates, algae, and fish) were collected from 47 sites in 2005-08. The types of samples and number of sites sampled varied annually due to drought and program constraints.

Current ecological conditions (2005-08) are described in this report for water quality, habitat, macroinvertebrates, algae, and fish communities. Water-quality data collected during water years 2005-08 indicated most constituents generally did not exceed acute or chronic criteria for the protection of aquatic life set by the States of Wyoming and Montana and the USEPA. Comparison of data from sites in Wyoming to WDEQ criteria indicated that $\mathrm{pH}$ and concentrations of selenium, iron, and manganese at a few sites in the Cheyenne and Belle Fourche River drainage basins exceeded criteria. Chloride concentrations exceeded the WDEQ chronic criterion in a few samples from the Little Powder River. Chloride concentrations were relatively high in the main-stem Powder River downstream from Salt Creek but did not exceed the WDEQ site-specific acute chloride criterion. Comparison of waterquality data from sites in Montana to MDEQ criteria indicated numerous exceedances for trace elements, particularly in samples from Pumpkin Creek and the main-stem Powder River at Moorhead and Locate, Montana.

Macroinvertebrate communities showed similarities within stream groups. Macroinvertebrate community composition at sites on the main-stem Tongue River and mountainous tributaries such as Clear Creek (TRMS group) were characterized by high Ephemeroptera taxa richness, high relative abundance of Ephemeroptera, dominance of the collectorgatherer feeding mode, and a high proportion of intolerant macroinvertebrates compared to communities at sites on the Tongue River plains tributaries (TRPT group) and main-stem Powder River (PRMS group). Among the Tongue River sites, the Tongue River above Hanging Woman Creek contained significantly $(\mathrm{p}<0.05)$ different (higher) relative abundance of noninsects than other sites on the main-stem Tongue River during 2005-08, perhaps due to the influence of Tongue River Reservoir. The relative abundance of the scraper feeding mode at sites on the main-stem Tongue River was significantly different (higher) in 2006 than in 2005 or 2007-08. Significant differences between 2006 and other years also were noted for metrics including Diptera taxa richness and relative abundance of Trichoptera at sites on the main-stem Powder River and some tributaries. Streamflow and climatic data indicated 2006 was a particularly severe year of a multiyear drought in the study area.

Macroinvertebrate community composition varied significantly $(\mathrm{p}<0.05)$ among sites on the main-stem Powder River during 2005-08. Macroinvertebrate community metric values from sites in the middle reach of the main-stem Powder River, from below Willow Creek to below Crazy Woman Creek, differed from metric values in the upper and lower reaches of the Powder River. Metric values for Ephemeroptera taxa richness, Ephemeroptera relative abundance, and intolerant macroinvertebrate relative abundance tended to be lower, whereas Diptera relative abundance and filterer-collector relative abundance tended to be higher at sites in the middle reach of the main-stem Powder River than at upstream and downstream sites on the main-stem Powder River. The affected metrics included measures of taxa richness, relative abundance, feeding modes, and tolerance, which were indicative of communitywide differences.

Algal samples from the main-stem Powder River generally confirmed the pattern observed in the macroinvertebrate communities. Algal communities in the middle reach of the Powder River often were characterized by dominance of a single taxon and low biovolume compared to other sites in the study area, which might be indicative of anthropogenic disturbances. Diatoms composed the majority of the taxa identified in 2005 and 2007, whereas green and blue-green algae commonly dominated in terms of relative abundance at sites throughout the study area.

Fish communities of the main-stem Tongue River and tributaries contained 29 fish species, of which 15 were native, during 2005-06 and 2008. Communities at the main-stem Tongue River sites were dominated by smallmouth bass, white sucker, yellow bullhead, and rock bass. Significant differences in fish Index of Biotic Integrity metrics from the Tongue River upstream from Hanging Woman Creek to those from other sites on the main-stem Tongue River might have been related to fish migration from Tongue River Reservoir. Fish Index of Biotic Integrity scores from upper Youngs Creek and upper Squirrel Creek were among the highest in the study area.

Fish communities of the main-stem Powder River and tributaries contained 24 fish species, of which 17 were native. The most abundant fish were native species including sand shiner and flathead chub. Species richness on the main-stem Powder River was highest downstream from Burger Draw, where 13 species were collected in 2008. Although a few significant differences in fish metrics along the main-stem Powder River were noted, the differences did not correspond to the pattern observed for the macroinvertebrate and algae communities. 
Potential causes of the anomalous pattern in macroinvertebrate communities of the middle reach of the main-stem Powder River include natural influences, discharge of production water from CBNG development, and other human activities. Relations between environmental variables and the macroinvertebrate taxonomy and metrics from the Powder River indicated some of the variation in the macroinvertebrate communities could be explained by variation in physical variables (specifically, turbidity, embeddedness, particle size, and streamflow) and chemical variables (alkalinity, $\mathrm{pH}$, and specific conductance). Of those environmental variables, alkalinity perhaps is the best indicator of CBNG development because of the sodium-bicarbonate signature of CBNG water. Mean alkalinity concentrations at the time of biological sampling were similar throughout the main-stem Powder River, with the exception of the site downstream from Burger Draw in Wyoming that had a relatively high mean alkalinity concentration, perhaps due to the influences of several CBNG discharges upstream. Given that macroinvertebrate communities integrate responses to environmental variables over a longer time period than instantaneous samples, it is likely that the macroinvertebrate communities in the middle Powder River reach between the Willow and Crazy Woman Creek confluences are responding to variation in the measured environmental variables beyond those observed at the time of sampling, or to other environmental variables that were not measured. The recent (2006-08) large increase in CBNG production in the Powder River drainage is a logical explanation for the observed patten in macroinvertebrate communities of the main-stem Powder River, but further study would be needed for confirmation.

The fish community composition of the main-stem Powder River observed in this study was similar to that observed by other investigators during about the same timeframe but may represent a departure from historical (1964-80) conditions. Potential causes for the departure include natural variation and the effects of resource development. Toxicity testing by other investigators indicates larval fish are more sensitive to sodium bicarbonate from CBNG discharges in the study area than the adult and juvenile fish that were sampled during this study.

In conclusion, the assessment of the current condition (2005-08) of biological communities in the Powder River structural basin indicated anomalous conditions for macroinvertebrate and algal communities in the middle reach of the Powder River compared to the upper and lower reaches. The anomalous conditions occurred at the lower trophic levels represented by macroinvertebrates and algae, whereas the higher trophic levels represented by the fish community generally did not vary significantly along the Powder River during 2005-08. Although CBNG development might have led to the observed patterns, further study of macroinvertebrate and algal communities in the middle reach of the Powder River is needed for confirmation. The sampling design proposed for further study is based on paired sampling locations, upstream and downstream from CBNG discharge points.

\section{Acknowledgments}

Seth Davidson, Chris Murray, Dan Sturgis, Tom Cleasby, and others with the USGS assisted with data collection in 2007-08. Many private landowners, as well as the Anadarko Corporation (The Woodlands, Texas), allowed access to sampling sites. Colleague reviewers were Daren Carlisle and Jill Frankforter, USGS. Eric Hargett (WDEQ), Dave Feldman (MDEQ), Rod McNeil (MDEQ), and several other representatives of ATG agencies provided helpful comments and discussion. Laura Hallberg, USGS, provided Geographic Information System (GIS) analyses.

\section{References}

Bahls, L.L., 1993, Periphyton bioassessment methods for Montana streams: Helena, Montana Department of Environmental Quality, 31 p. plus appendixes.

Barbour, M.T., Gerritsen, J., Snyder, B.D., and Stribling, J.B., 1999, Rapid bioassessment protocols for use in streams and wadeable rivers-Periphyton, benthic macroinvertebrates, and fish ( $2 \mathrm{~d}$ ed.): U.S. Environmental Protection Agency, Office of Water, EPA 841-B-99-002, variable pagination.

Baxter, G.T., and Stone, M.D., 1995, Fishes of Wyoming: Cheyenne, Wyoming Game and Fish Department Bulletin no. 4, 290 p.

Bramblett, R.G., Johnson, T.R., Zale, A.V., and Heggem, D.G., 2005, Development and evaluation of a fish assemblage index of biotic integrity for northwestern Great Plains streams: Transactions of the American Fisheries Society, v. 134, p. 624-640.

Bray, J.R., and Curtis, J.T., 1957, An ordination of the upland forest communities of southern Wisconsin: Ecological Monographs, v. 27, p. 325-349.

Brinck, E.L, Drever, J.I., and Frost, C.D., 2008, The geochemical evolution of water coproduced with coalbed natural gas in the Powder River Basin, Wyoming: Environmental Geosciences, v. 15, no. 4, p. 153-171.

Brown, T.H., and Johnston, Chris, 2005, Assessment of the environmental impacts of coalbed natural gas development in the Powder River Basin-Use of coalbed natural gasproduced water for cropland irrigation, in Zoback, M.D., ed., Western Resources Project final report—Produced groundwater associated with coalbed natural gas production in the Powder River Basin: Wyoming State Geological Survey Report of Investigations no. 55, chap. 3, p. 71-85. 
Bureau of Land Management, 2003, Record of decision and resource management plan amendments for the Powder River Basin oil and gas project, WY-070-02-065, Buffalo Field Office, Wyoming, April 2003 [variously paged], accessed February 16, 2010, at http://www.blm.gov/ pgdata/etc/medialib/blm/wy/information/NEPA/ prb-rod.Par.71614.File.dat/00rod.pdf.

Bureau of Land Management, 2009, Powder River Basin Interagency Working Group, accessed on September 21, 2009, at http://www.wy.blm.gov/prbgroup/.

Clark, M.L., and Mason, J.P., 2007, Water-quality characteristics for sites in the Tongue, Powder, Cheyenne, and Belle Fourche River drainage basins, Wyoming and Montana, water years 2001-05, with temporal patterns of selected long-term water-quality data: U.S. Geological Survey Scientific Investigations Report 2007-5146, 65 p.

Clarke, K.R., and Gorley, R.N., 2006, PRIMER v6: Plymouth, United Kingdom, Plymouth Marine Laboratory, PRIMER-E Ltd., 190 p.

Cuffney, T.C., 2003, User's manual for the National WaterQuality Assessment Program invertebrate data analysis system (IDAS) software-Version 3: U.S. Geological Survey Open-File Report 2003-172, 103 p.

Davis, W.N., 2008, Effects of coalbed natural gas development on fish assemblages in tributary streams in Powder River Basin, Montana and Wyoming: Bozeman, Montana State University, Master's thesis, 137 p., 26 figs.

Davis, W.N., Bramblett, R.G., Zale, A.V., and Endicott, C.L., 2009, A review of the potential effects of coal bed natural gas development activities on fish assemblages of the Powder River Geologic Basin: Reviews in Fisheries Science, v. 17 , no. 3 , p. $402-422$.

Helsel, D.R., and Hirsch, R.M., 2002, Statistical methods in water resources: U.S. Geological Survey Techniques of Water Resources Investigations, book 4, chap. A3, 522 p.

Hilsenhoff, W.L., 1987, An improved index of organic stream pollution: Great Lakes Entomologist, v. 20, p. 31-39.

Hubert, W. A., 1993, The Powder River: a relatively pristine stream on the Great Plains, in Hesse, L.W., Stalnaker, C.B. Benson, N.G. and Zuboy, J.R., eds., Restoration planning for the rivers of the Mississippi River ecosystem: Washington, D.C., National Biological Survey, Biological Report 19, p. 387-395.

Lowry, M.E., Wilson, J.F., and others, 1986, Hydrology of Area 50, Northern Great Plains and Rocky Mountain Coal Provinces, Wyoming and Montana: U.S. Geological Survey Water-Resources Investigations Open-File Report 83-545, $137 \mathrm{p}$.
Montana Department of Environmental Quality, 2008, Montana numeric water quality standards: Montana Department of Environmental Quality, Circular DEQ-7, 42 p., accessed May 27, 2010, at http://www.deq.mt.gov/wqinfo/Standards/ PDF/CompiledDEQ7.pdf.

Moulton, S.R., II, Kennen, J.G., Goldstein, R.M., and Hambrook, J.A., 2002, Revised protocols for sampling algal, invertebrate, and fish communities as part of the National Water-Quality Assessment Program: U.S. Geological Survey Open-File Report 2002-150, 75 p.

National Oceanic and Atmospheric Administration, Satellite and Information Service, 2005, Climate of 2005June, Wyoming Division 4 Drought, Regional overview/ paleo perspective, accessed October 26, 2007, at http://www.ncdc.noaa.gov/oa/climate/research/2005/jun/ st048dv04pcp200506.html.

Peterson, D.A., and Porter, S.D., 2002, Biological and chemical indicators of eutrophication in the Yellowstone River and major tributaries during August, 2000: National Water Quality Monitoring Council, Proceedings of the 2002 National Water Quality Monitoring Conference, Madison, Wisc., May 20-23, 14 p.

Peterson, D.A., Wright, P.R., Edwards, G.P., Jr., Hargett, E.G., Feldman, D.L., Zumberge, J.R., and Dey, Paul, 2009, Ecological assessment of streams in the Powder River structural basin, Wyoming and Montana, 2005-06: U.S. Geological Survey Scientific Investigations Report 2009-5023, 139 p.

Porter, S.D., 2008, Algal attributes-An autecological classification of algal taxa collected by the National Water-Quality Assessment Program: U.S. Geological Survey Data Series 329, http://pubs.usgs.gov/ds/ds329.

Potapova, M., and Charles, D.F., 2005, Choice of substrate in algae-based water-quality assessment: Journal of the North American Benthological Society, v. 24, no. 2, p. 415-427.

Powder River Natural Gas Interagency Working Group, 2004, Charter: Buffalo, Wyo., Bureau of Land Management, 15 p., accessed April 1, 2008, at http://www.wy.blm.gov/prbgroup/ pdfs/charter061504.pdf.

Rice, C.A., Ellis, M.S., and Bullock, J.H., Jr., 2000, Water coproduced with coalbed methane in the Powder River Basin, Wyoming - Preliminary compositional data: U.S. Geological Survey Open-File Report 2000-372, 20 p.

Ringen, B.H., and Daddow, P.B., 1990, Hydrology of the Powder River alluvium between Sussex, Wyoming, and Moorhead, Montana: U.S. Geological Survey Water-Resources Investigations Report 89-4002, 48 p. 
Senecal, A.C., 2009, Fish assemblage structure and flow regime of the Powder River, Wyoming - An assessment of the potential effects of flow augmentation related to energy development: Laramie, University of Wyoming, Master's thesis, 237 p., 37 figs.

Skaar, Don, Farag, A.M., and Harper, D., 2006, Toxicity of sodium bicarbonate to fish from coal-bed natural gas production in the Tongue and Powder River drainages, Montana and Wyoming: U.S. Geological Survey Fact Sheet 2006-3092, 4 p.

Smith, R.L., Repert, D.A., and Hart, C.P., 2009, Geochemistry of inorganic nitrogen in waters released from coal-bed natural gas production wells in the Powder River Basin, Wyoming: Environmental Science and Technology, v. 43, no. 7 , p. $2348-2354$.

Stevenson, R.J., Bothwell, M.L., and Lowe, R.L., 1996, Algal ecology: San Diego, Calif., Academic Press, 753 p.

Stricker, G.D., Flores, R.M., McGarry, D.E., Stillwell, D.P., Hoppe, D.J., Stillwell, C.R., Ochs, A.M., Ellis, M.S., Osvald, K.S., Taylor, S.L., Thorvaldson, M.C., Trippi, M.H., Grose, S.D., Crockett, F.J., and Shariff, A.J., 2006, Gas desorption and adsorption isotherm studies of coals in the Powder River Basin, Wyoming and adjacent basins in Wyoming and North Dakota: U.S. Geological Survey Open-File Report 2006-1174, 21 p., accessed September 21, 2009, at http://pubs.usgs.gov/of/2006/1174/.

TIBCO Software Inc., 2008, Tibco Spotfire S+ version 8.1.1: Palo Alto, California.

U.S. Environmental Protection Agency, 1988, Ambient water quality criteria for chloride: Office of Water, EPA 440/588-001, February 1988, 39 p., accessed November 15, 2009, at http://www.epa.gov/waterscience/criteria/library/ ambientwqc/chloride1988.pdf.

U.S. Environmental Protection Agency, 2004, Draft aquatic life water quality criteria for selenium-2004: Office of Water, EPA-822-D-04-001, accessed November 15, 2009, at $h t t p: / / w w w . e p a . g o v /$ waterscience/criteria/selenium/pdfs/ complete.pdf.
U.S. Environmental Protection Agency, 2005, National recommended water quality criteria, accessed November 15, 2009, at http://www.epa.gov/waterscience/criteria/wqctable/index.html.

U.S. Environmental Protection Agency, 2007, Aquatic life ambient freshwater quality criteria - copper: Office of Water, EPA-822-R-07-001, February 2007, accessed November 15, 2009, at http://www.epa.gov/waterscience/ criteria/copper/2007/criteria-full.pdf.

Walsh, S.J., and Meador, M.R., 1998, Guidelines for quality assurance and quality control of fish taxonomic data collected as part of the National Water-Quality Assessment Program: U.S. Geological Survey Water-Resources Investigations Report 98-4239, 33 p.

Wangsness, D.J., and Peterson, D.A., 1980, Behavioral and catastrophic drift of invertebrates in two streams in northeastern Wyoming: U.S. Geological Survey Open-File Report 80-1101, 13 p.

Wolman, 1954, A method for sampling coarse riverbed material: Transactions of the American Geophysical Union, v. 35 , p. $951-956$.

Wyoming Department of Environmental Quality, 2007, Water quality rules and regulations, chapter 1 , Wyoming surface water quality standards: Wyoming Department of Environmental Quality, Water Quality Division, [variously paged], accessed November 15, 2009, at http://soswy.state.wy.us/ Rules/RULES/6547.pdf.

Wyoming Department of Environmental Quality, 2009, National Pollutant Discharge Elimination System (NPDES) Program; Wyoming Department of Environmental Quality database, accessed January 28, 2009, at http://deq.state.wy.us/ wqd/npdes/.

Wyoming Oil and Gas Conservation Commission, 2010, Coal bed methane wells: digital data, accessed February 8, 2010, at: http://wogcc.state.wy.us/. 
Appendix. Summary statistics for water-quality constituents, Powder River structural basin, Wyoming and Montana, water years 2005-08.

[--, value cannot be calculated; $\mathrm{mg} / \mathrm{L}$, milligrams per liter; $\mu \mathrm{S} / \mathrm{cm}$, microsiemens per centimeter at 25 degrees Celsius; deg C, degrees Celsius; $\mathrm{CaCO}_{3}$, calcium carbonate; as $\mathrm{N}$, as nitrogen; as $\mathrm{P}$, as phosphorus; $\mu \mathrm{g} / \mathrm{L}$, micrograms per liter; $\mathrm{ng} / \mathrm{L}$, nanograms per liter; mm, millimeters; *, constituent statistics were estimated using a log-probability regression; **, too many non-detected constituent concentrations to estimate statistics with log-probablity regression]

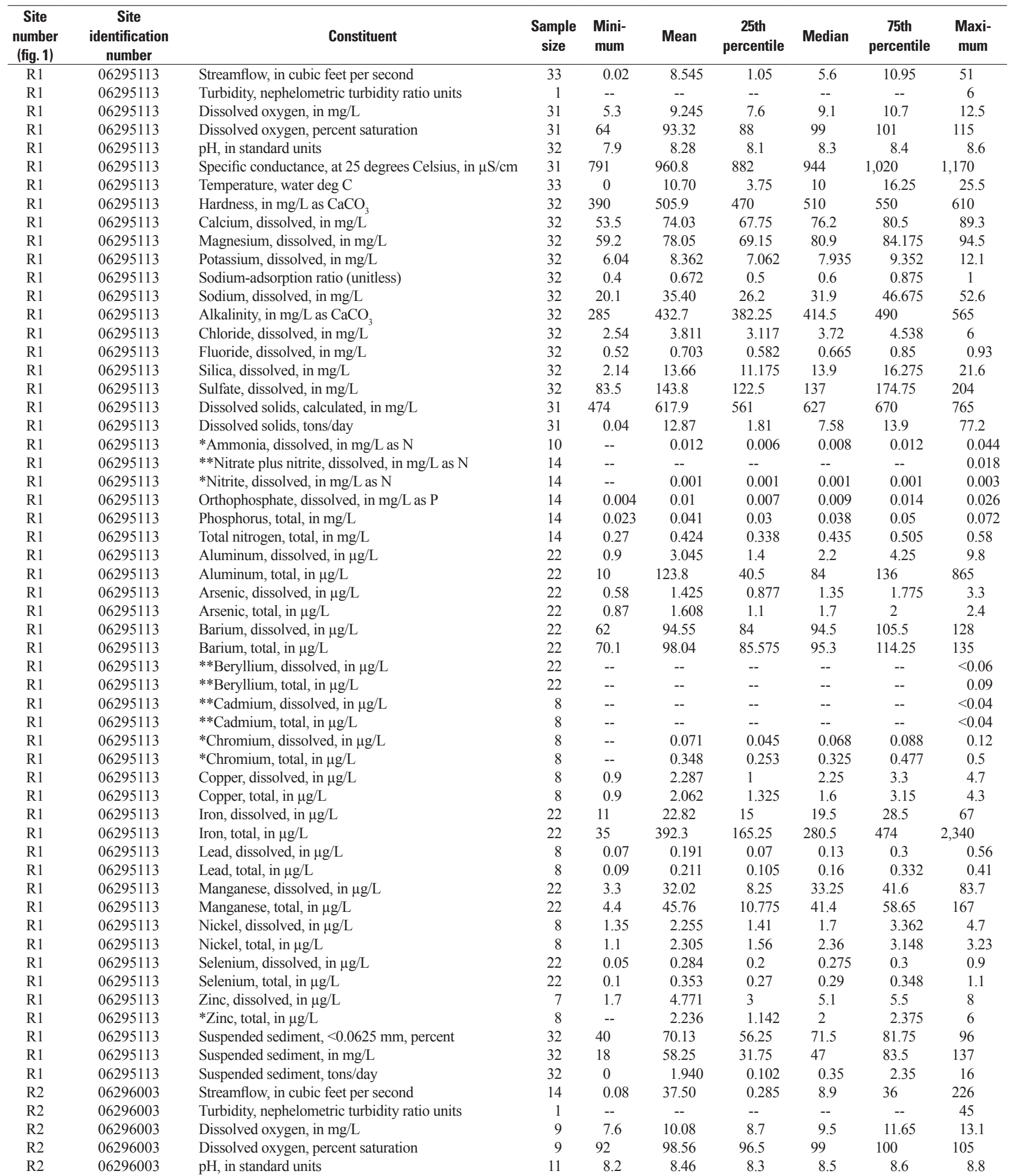


Appendix. Summary statistics for water-quality constituents, Powder River structural basin, Wyoming and Montana, water years 2005-08.-Continued

[--, value cannot be calculated; $\mathrm{mg} / \mathrm{L}$, milligrams per liter; $\mu \mathrm{S} / \mathrm{cm}$, microsiemens per centimeter at 25 degrees Celsius; deg $\mathrm{C}$, degrees $\mathrm{Celsius}^{\mathrm{C}} \mathrm{CaCO}_{3}$, calcium carbonate; as $\mathrm{N}$, as nitrogen; as $\mathrm{P}$, as phosphorus; $\mu \mathrm{g} / \mathrm{L}$, micrograms per liter; $\mathrm{ng} / \mathrm{L}$, nanograms per liter; mm, millimeters; *, constituent statistics were estimated using a log-probability regression; **, too many non-detected constituent concentrations to estimate statistics with log-probablity regression]

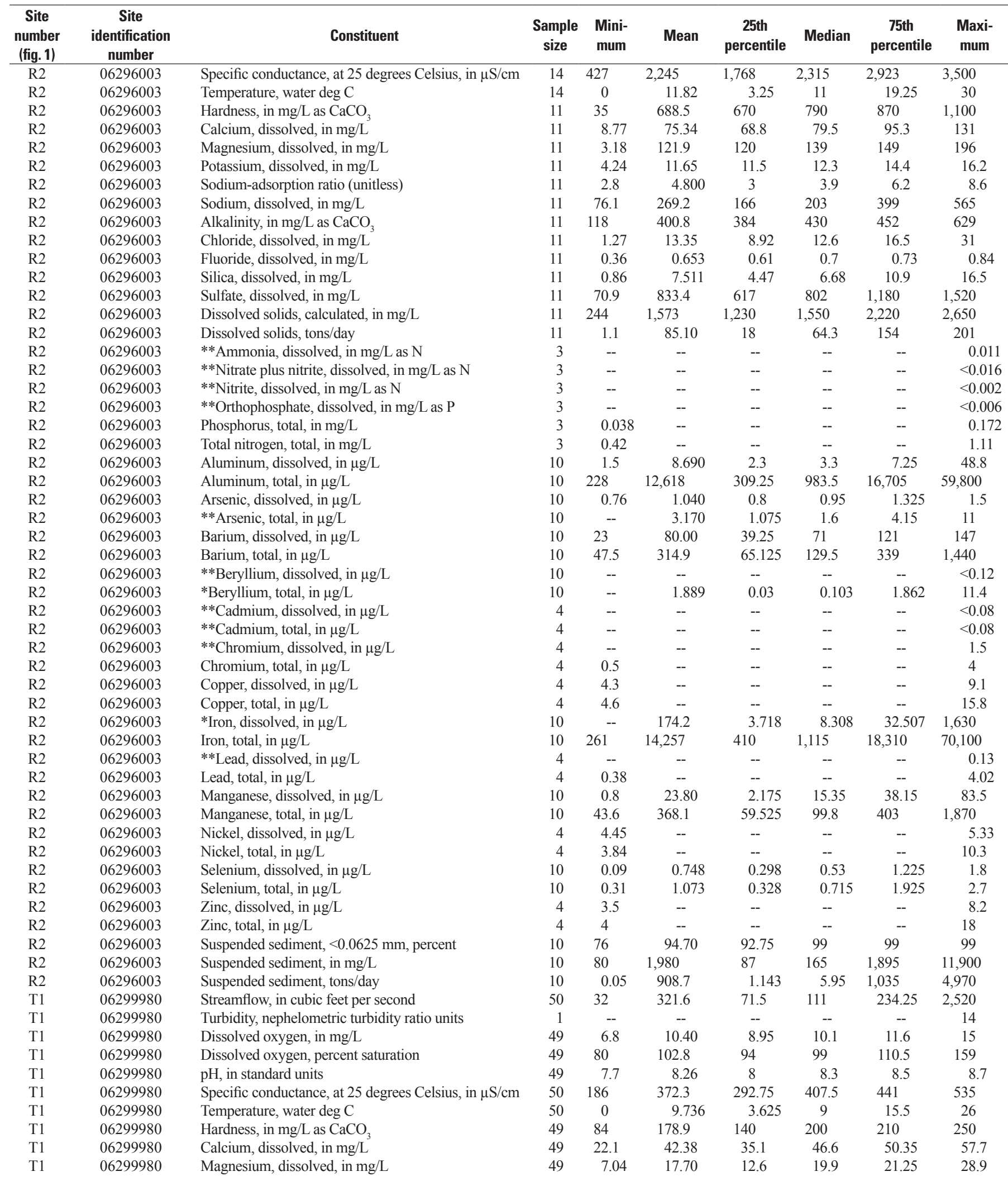


Appendix. Summary statistics for water-quality constituents, Powder River structural basin, Wyoming and Montana, water years 2005-08.-Continued

[--, value cannot be calculated; $\mathrm{mg} / \mathrm{L}$, milligrams per liter; $\mu \mathrm{S} / \mathrm{cm}$, microsiemens per centimeter at 25 degrees Celsius; deg $\mathrm{C}$, degrees Celsius; $\mathrm{CaCO}_{3}$, calcium carbonate; as $\mathrm{N}$, as nitrogen; as $\mathrm{P}$, as phosphorus; $\mu \mathrm{g} / \mathrm{L}$, micrograms per liter; ng/L, nanograms per liter; mm, millimeters; *, constituent statistics were estimated using a log-probability regression; **, too many non-detected constituent concentrations to estimate statistics with log-probablity regression]

\begin{tabular}{|c|c|c|c|c|c|c|c|c|c|}
\hline $\begin{array}{c}\text { Site } \\
\text { number } \\
\text { (fig. 1) }\end{array}$ & $\begin{array}{c}\text { Site } \\
\text { identification } \\
\text { number } \\
\end{array}$ & Constituent & $\begin{array}{c}\text { Sample } \\
\text { size }\end{array}$ & $\begin{array}{l}\text { Mini- } \\
\text { mum }\end{array}$ & Mean & $\begin{array}{c}\text { 25th } \\
\text { percentile }\end{array}$ & Median & $\begin{array}{c}\text { 75th } \\
\text { percentile }\end{array}$ & $\begin{array}{c}\text { Maxi- } \\
\text { mum }\end{array}$ \\
\hline T1 & 06299980 & Potassium, dissolved, in $\mathrm{mg} / \mathrm{L}$ & 49 & 0.72 & 1.543 & 1.175 & 1.37 & 1.665 & 4.2 \\
\hline T1 & 06299980 & Sodium-adsorption ratio (unitless) & 49 & 0.1 & 0.318 & 0.2 & 0.3 & 0.4 & 0.7 \\
\hline $\mathrm{T} 1$ & 06299980 & Sodium, dissolved, in mg/L & 49 & 2.45 & 9.867 & 6 & 9.98 & 12.65 & 25.5 \\
\hline $\mathrm{T} 1$ & 06299980 & Alkalinity, in $\mathrm{mg} / \mathrm{L}$ as $\mathrm{CaCO}_{3}$ & 49 & 81 & 158.0 & 125.5 & 178 & 187 & 212 \\
\hline $\mathrm{T} 1$ & 06299980 & Chloride, dissolved, in $\mathrm{mg} / \mathrm{L}^{3}$ & 49 & 0.54 & 1.442 & 1.04 & 1.35 & 1.75 & 3.6 \\
\hline $\mathrm{T} 1$ & 06299980 & Fluoride, dissolved, in $\mathrm{mg} / \mathrm{L}$ & 49 & 0.08 & 0.162 & 0.14 & 0.17 & 0.19 & 0.23 \\
\hline T1 & 06299980 & Silica, dissolved, in $\mathrm{mg} / \mathrm{L}$ & 49 & 3.63 & 6.464 & 5.29 & 6.58 & 7.55 & 9.54 \\
\hline T1 & 06299980 & Sulfate, dissolved, in $\mathrm{mg} / \mathrm{L}$ & 49 & 8.26 & 41.52 & 22.55 & 44.3 & 55.25 & 110 \\
\hline $\mathrm{T} 1$ & 06299980 & Dissolved solids, calculated, in $\mathrm{mg} / \mathrm{L}$ & 46 & 103 & 213.4 & 160.5 & 235 & 255 & 332 \\
\hline $\mathrm{T} 1$ & 06299980 & Dissolved solids, tons/day & 46 & 25.3 & 150.0 & 54.8 & 70.8 & 126 & 1,140 \\
\hline $\mathrm{T} 1$ & 06299980 & Dissolved solids, residue on evaporation, in $\mathrm{mg} / \mathrm{L}$ & 11 & 114 & 231.0 & 187 & 252 & 274 & 310 \\
\hline $\mathrm{T} 1$ & 06299980 & *Ammonia, dissolved, in $\mathrm{mg} / \mathrm{L}$ as $\mathrm{N}$ & 18 & -- & 0.013 & 0.005 & 0.007 & 0.012 & 0.085 \\
\hline T1 & 06299980 & *Nitrate plus nitrite, dissolved, in $\mathrm{mg} / \mathrm{L}$ as $\mathrm{N}$ & 18 & -- & 0.048 & 0.008 & 0.016 & 0.064 & 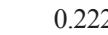 \\
\hline T1 & 06299980 & *Nitrite, dissolved, in $\mathrm{mg} / \mathrm{L}$ as $\mathrm{N}$ & 18 & -- & 0.002 & 0.001 & 0.001 & 0.002 & 0.004 \\
\hline T1 & 06299980 & *Orthophosphate, dissolved, in $\mathrm{mg} / \mathrm{L}$ as $\mathrm{P}$ & 18 & -- & 0.009 & 0.003 & 0.005 & 0.009 & 0.039 \\
\hline T1 & 06299980 & Phosphorus, total, in $\mathrm{mg} / \mathrm{L}$ & 18 & 0.005 & 0.045 & 0.009 & 0.018 & 0.053 & 0.26 \\
\hline T1 & 06299980 & Total nitrogen, total, in $\mathrm{mg} / \mathrm{L}$ & 18 & 0.1 & 0.337 & 0.175 & 0.235 & 0.39 & 1.21 \\
\hline T1 & 06299980 & *Aluminum, dissolved, in $\mu \mathrm{g} / \mathrm{L}$ & 18 & -- & 2.309 & 1.252 & 1.628 & 2.425 & 9.6 \\
\hline $\mathrm{T} 1$ & 06299980 & Aluminum, total, in $\mu \mathrm{g} / \mathrm{L}$ & 29 & 22 & 279.8 & 34.5 & 52 & 204.5 & 2,810 \\
\hline T1 & 06299980 & Arsenic, dissolved, in $\mu \mathrm{g} / \mathrm{L}$ & 29 & 0.2 & 0.408 & 0.3 & 0.35 & 0.5 & 0.8 \\
\hline T1 & 06299980 & $*$ Arsenic, total, in $\mu \mathrm{g} / \mathrm{L}$ & 29 & -- & 0.66 & 0.457 & 0.56 & 0.755 & 2 \\
\hline $\mathrm{T} 1$ & 06299980 & Barium, dissolved, in $\mu \mathrm{g} / \mathrm{L}$ & 18 & 25 & 43.11 & 41 & 44 & 47.25 & 57 \\
\hline T1 & 06299980 & Barium, total, in $\mu \mathrm{g} / \mathrm{L}$ & 29 & 32.9 & 47.39 & 41.15 & 44.3 & 48.7 & 90.2 \\
\hline T1 & 06299980 & **Beryllium, dissolved, in $\mu \mathrm{g} / \mathrm{L}$ & 18 & -- & -- & -- & -- & -- & $<0.06$ \\
\hline T1 & 06299980 & *Beryllium, total, in $\mu \mathrm{g} / \mathrm{L}$ & 29 & -- & 0.044 & 0.015 & 0.029 & 0.046 & 0.32 \\
\hline $\mathrm{T} 1$ & 06299980 & Boron, dissolved, in $\mu \mathrm{g} / \mathrm{L}$ & 15 & 15 & 32.53 & 30 & 31 & 35 & 53 \\
\hline T1 & 06299980 & **Cadmium, dissolved, in $\mu \mathrm{g} / \mathrm{L}$ & 16 & -- & -- & -- & -- & -- & $<0.04$ \\
\hline $\mathrm{T} 1$ & 06299980 & $* *$ Cadmium, total, in $\mu \mathrm{g} / \mathrm{L}$ & 16 & -- & -- & -- & -- & -- & 0.12 \\
\hline $\mathrm{T} 1$ & 06299980 & Chromium, dissolved, in $\mu \mathrm{g} / \mathrm{L}$ & 1 & -- & -- & -- & -- & -- & 0.13 \\
\hline T1 & 06299980 & ${ }^{*}$ Chromium, total, in $\mu \mathrm{g} / \mathrm{L}$ & 16 & -- & 1.172 & 0.881 & 1 & 1.251 & 3 \\
\hline $\mathrm{T} 1$ & 06299980 & Copper, dissolved, in $\mu \mathrm{g} / \mathrm{L}$ & 13 & 0.7 & 1.033 & 0.8 & 0.9 & 1.2 & 1.8 \\
\hline $\mathrm{T} 1$ & 06299980 & Copper, total, in $\mu \mathrm{g} / \mathrm{L}$ & 16 & 0.7 & 1.663 & 1 & 1.15 & 1.7 & 6.9 \\
\hline $\mathrm{T} 1$ & 06299980 & Iron, dissolved, in $\mu \mathrm{g} / \mathrm{L}$ & 29 & 6 & 20.21 & 13 & 17 & 29.5 & 41 \\
\hline T1 & 06299980 & Iron, total, in $\mu \mathrm{g} / \mathrm{L}$ & 18 & 55 & 439.7 & 86.75 & 175.5 & 510 & 2,450 \\
\hline $\mathrm{T} 1$ & 06299980 & *Lead, dissolved, in $\mu \mathrm{g} / \mathrm{L}$ & 14 & -- & 0.092 & 0.053 & 0.065 & 0.085 & 0.43 \\
\hline $\mathrm{T} 1$ & 06299980 & Lead, total, in $\mu \mathrm{g} / \mathrm{L}$ & 16 & 0.04 & 0.569 & 0.06 & 0.125 & 0.4 & 5.43 \\
\hline T1 & 06299980 & Lithium, dissolved, in $\mu \mathrm{g} / \mathrm{L}$ & 15 & 3.9 & 9.707 & 8.5 & 9.5 & 10.3 & 16.6 \\
\hline T1 & 06299980 & Manganese, dissolved, in $\mu \mathrm{g} / \mathrm{L}$ & 29 & 1.8 & 9.948 & 5.2 & 6.1 & 10 & 34.1 \\
\hline T1 & 06299980 & Manganese, total, in $\mu \mathrm{g} / \mathrm{L}$ & 18 & 3.1 & 27.51 & 8.1 & 22.75 & 43.825 & 86.6 \\
\hline $\mathrm{T} 1$ & 06299980 & Mercury, total, ng/L & 5 & 0.28 & -- & -- & -- & -- & 3.8 \\
\hline T1 & 06299980 & $* *$ Mercury, total, $\mu \mathrm{g} / \mathrm{L}$ & 3 & -- & -- & -- & -- & -- & $<0.01$ \\
\hline T1 & 06299980 & Nickel, dissolved, in $\mu \mathrm{g} / \mathrm{L}$ & 15 & 0.23 & 1.163 & 0.47 & 1.09 & 1.79 & 2.27 \\
\hline $\mathrm{T} 1$ & 06299980 & Nickel, total, in $\mu \mathrm{g} / \mathrm{L}$ & 16 & 0.85 & 1.656 & 0.923 & 1.35 & 2.003 & 5.34 \\
\hline T1 & 06299980 & *Selenium, dissolved, in $\mu \mathrm{g} / \mathrm{L}$ & 18 & -- & 0.282 & 0.168 & 0.225 & 0.305 & 0.8 \\
\hline T1 & 06299980 & Selenium, total, in $\mu \mathrm{g} / \mathrm{L}$ & 29 & 0.08 & 0.301 & 0.18 & 0.21 & 0.32 & 1.2 \\
\hline T1 & 06299980 & Strontium, dissolved, in $\mu \mathrm{g} / \mathrm{L}$ & 15 & 93 & 207.3 & 189 & 217 & 238 & 253 \\
\hline $\mathrm{T} 1$ & 06299980 & *Zinc, dissolved, in $\mu \mathrm{g} / \mathrm{L}$ & 14 & -- & 1.279 & 0.467 & 0.755 & 2.175 & 3.3 \\
\hline $\mathrm{T} 1$ & 06299980 & $*$ Zinc, total, in $\mu \mathrm{g} / \mathrm{L}$ & 16 & -- & 2.864 & 1 & 1.224 & 2.75 & 19 \\
\hline T1 & 06299980 & Suspended sediment, $<0.0625 \mathrm{~mm}$, percent & 33 & 36 & 85.67 & 78.5 & 91 & 95 & 99 \\
\hline T1 & 06299980 & Suspended sediment, in $\mathrm{mg} / \mathrm{L}$ & 33 & 4 & 32.15 & 9.5 & 17 & 35 & 266 \\
\hline $\mathrm{T} 1$ & 06299980 & Suspended sediment, tons/day & 33 & 0.65 & 47.46 & 1.95 & 3.7 & 18 & 826 \\
\hline T2 & 06305700 & Streamflow, in cubic feet per second & 38 & 5.1 & 200.5 & 42.75 & 67.5 & 159 & 1,350 \\
\hline T2 & 06305700 & Turbidity, nephelometric turbidity ratio units & 2 & 3.8 & -- & -- & -- & -- & 4.5 \\
\hline $\mathrm{T} 2$ & 06305700 & Dissolved oxygen, in $\mathrm{mg} / \mathrm{L}$ & 38 & 6 & 10.86 & 8.925 & 10.5 & 12.875 & 15.9 \\
\hline T2 & 06305700 & Dissolved oxygen, percent saturation & 38 & 62 & 113.2 & 95.5 & 104.5 & 126 & 194 \\
\hline $\mathrm{T} 2$ & 06305700 & $\mathrm{pH}$, in standard units & 37 & 7.8 & 8.31 & 8.05 & 8.3 & 8.55 & 9 \\
\hline $\mathrm{T} 2$ & 06305700 & Specific conductance, at 25 degrees Celsius, in $\mu \mathrm{S} / \mathrm{cm}$ & 38 & 130 & 595.6 & 539 & 663 & 723.75 & 860 \\
\hline T2 & 06305700 & Temperature, water deg C & 38 & 0 & 11.85 & 5.25 & 10.75 & 19.175 & 28 \\
\hline T2 & 06305700 & Hardness, in $\mathrm{mg} / \mathrm{L}$ as $\mathrm{CaCO}_{3}$ & 37 & 57 & 281.9 & 250 & 320 & 350 & 420 \\
\hline T2 & 06305700 & Calcium, dissolved, in $\mathrm{mg} / \mathrm{L}$ & 37 & 13.2 & 54.05 & 49.85 & 61.1 & 67.25 & 76.2 \\
\hline
\end{tabular}


Appendix. Summary statistics for water-quality constituents, Powder River structural basin, Wyoming and Montana, water years 2005-08. - Continued

[--, value cannot be calculated; $\mathrm{mg} / \mathrm{L}$, milligrams per liter; $\mu \mathrm{S} / \mathrm{cm}$, microsiemens per centimeter at 25 degrees Celsius; deg $\mathrm{C}$, degrees Celsius; $\mathrm{CaCO}_{3}$, calcium carbonate; as $\mathrm{N}$, as nitrogen; as $\mathrm{P}$, as phosphorus; $\mu \mathrm{g} / \mathrm{L}$, micrograms per liter; $\mathrm{ng} / \mathrm{L}$, nanograms per liter; mm, millimeters; *, constituent statistics were estimated using a log-probability regression; **, too many non-detected constituent concentrations to estimate statistics with log-probablity regression]

\begin{tabular}{|c|c|c|c|c|c|c|c|c|c|}
\hline $\begin{array}{c}\text { Site } \\
\text { number } \\
\text { (fig. 1) }\end{array}$ & $\begin{array}{c}\text { Site } \\
\text { identification } \\
\text { number }\end{array}$ & Constituent & $\begin{array}{c}\text { Sample } \\
\text { size }\end{array}$ & $\begin{array}{l}\text { Mini- } \\
\text { mum }\end{array}$ & Mean & $\begin{array}{c}\text { 25th } \\
\text { percentile }\end{array}$ & Median & $\begin{array}{c}\text { 75th } \\
\text { percentile }\end{array}$ & $\begin{array}{l}\text { Maxi- } \\
\text { mum }\end{array}$ \\
\hline $\mathrm{T} 2$ & 06305700 & Magnesium, dissolved, in $\mathrm{mg} / \mathrm{L}$ & 37 & 5.76 & 35.51 & 29.8 & 39.6 & 44.65 & 56.6 \\
\hline $\mathrm{T} 2$ & 06305700 & Sodium, dissolved, in $\mathrm{mg} / \mathrm{L}$ & 37 & 3.96 & 23.36 & 18.35 & 24.7 & 30.25 & 48.2 \\
\hline $\mathrm{T} 2$ & 06305700 & Alkalinity, in $\mathrm{mg} / \mathrm{L}$ as $\mathrm{CaCO}_{3}$ & 37 & 50 & 208.0 & 181.5 & 236 & 257.5 & 300 \\
\hline $\mathrm{T} 2$ & 06305700 & Chloride, dissolved, in $\mathrm{mg} / \mathrm{L}$ & 37 & 0.88 & 6.786 & 4.685 & 6.39 & 8.905 & 18.2 \\
\hline $\mathrm{T} 2$ & 06305700 & Sulfate, dissolved, in $\mathrm{mg} / \mathrm{L}$ & 37 & 13.8 & 111.3 & 95.4 & 118 & 138.5 & 210 \\
\hline $\mathrm{T} 2$ & 06305700 & Dissolved solids, calculated, in mg/L & 36 & 77 & 367.4 & 313 & 412.5 & 451.5 & 559 \\
\hline $\mathrm{T} 2$ & 06305700 & Dissolved solids, tons/day & 36 & 7.7 & 122.9 & 55.45 & 73.3 & 159.75 & 914 \\
\hline $\mathrm{T} 2$ & 06305700 & *Ammonia, dissolved, in $\mathrm{mg} / \mathrm{L}$ as $\mathrm{N}$ & 17 & -- & 0.024 & 0.007 & 0.012 & 0.023 & 0.11 \\
\hline $\mathrm{T} 2$ & 06305700 & *Nitrate plus nitrite, dissolved, in $\mathrm{mg} / \mathrm{L}$ as $\mathrm{N}$ & 17 & -- & 0.208 & 0.017 & 0.139 & 0.326 & 1.04 \\
\hline T2 & 06305700 & *Nitrite, dissolved, in $\mathrm{mg} / \mathrm{L}$ as $\mathrm{N}$ & 17 & -- & 0.007 & 0.001 & 0.003 & 0.007 & 0.033 \\
\hline $\mathrm{T} 2$ & 06305700 & Arsenic, dissolved, in $\mu \mathrm{g} / \mathrm{L}$ & 17 & 0.27 & 0.575 & 0.38 & 0.51 & 0.785 & 1.2 \\
\hline $\mathrm{T} 2$ & 06305700 & *Arsenic, total, in $\mu \mathrm{g} / \mathrm{L}$ & 17 & -- & 0.893 & 0.56 & 0.72 & 1.148 & 2 \\
\hline $\mathrm{T} 2$ & 06305700 & Barium, dissolved, in $\mu \mathrm{g} / \mathrm{L}$ & 17 & 18 & 49.71 & 48 & 52 & 55 & 60 \\
\hline $\mathrm{T} 2$ & 06305700 & Barium, total, in $\mu \mathrm{g} / \mathrm{L}$ & 17 & 25.1 & 53.62 & 50.15 & 53.6 & 60.05 & 67.7 \\
\hline T2 & 06305700 & **Beryllium, dissolved, in $\mu \mathrm{g} / \mathrm{L}$ & 17 & -- & -- & -- & -- & -- & 0.1 \\
\hline $\mathrm{T} 2$ & 06305700 & $* *$ Beryllium, total, in $\mu \mathrm{g} / \mathrm{L}$ & 17 & -- & -- & -- & -- & -- & 0.22 \\
\hline T2 & 06305700 & Boron, dissolved, in $\mu \mathrm{g} / \mathrm{L}$ & 14 & 46 & 83.79 & 68 & 80.5 & 97 & 131 \\
\hline T2 & 06305700 & **Cadmium, dissolved, in $\mu \mathrm{g} / \mathrm{L}$ & 15 & -- & -- & -- & -- & -- & $<0.04$ \\
\hline T2 & 06305700 & *Cadmium, total, in $\mu \mathrm{g} / \mathrm{L}$ & 15 & -- & 0.026 & 0.01 & 0.019 & 0.03 & 0.1 \\
\hline $\mathrm{T} 2$ & 06305700 & Chromium, dissolved, in $\mu \mathrm{g} / \mathrm{L}$ & 1 & -- & -- & -- & -- & -- & 0.15 \\
\hline $\mathrm{T} 2$ & 06305700 & *Chromium, total, in $\mu \mathrm{g} / \mathrm{L}$ & 15 & -- & 1.523 & 1 & 1.015 & 2 & 4 \\
\hline $\mathrm{T} 2$ & 06305700 & Copper, dissolved, in $\mu \mathrm{g} / \mathrm{L}$ & 15 & 0.69 & 1.354 & 1.1 & 1.3 & 1.6 & 2.2 \\
\hline $\mathrm{T} 2$ & 06305700 & Copper, total, in $\mu \mathrm{g} / \mathrm{L}$ & 15 & 1.2 & 2.347 & 1.5 & 1.9 & 2.7 & 6.7 \\
\hline $\mathrm{T} 2$ & 06305700 & **Mercury, total, $\mu \mathrm{g} / \mathrm{L}$ & 3 & -- & -- & -- & -- & -- & $<0.01$ \\
\hline $\mathrm{T} 2$ & 06305700 & Nickel, dissolved, in $\mu \mathrm{g} / \mathrm{L}$ & 14 & 0.52 & 1.822 & 0.89 & 1.86 & 2.388 & 3.32 \\
\hline $\mathrm{T} 2$ & 06305700 & Nickel, total, in $\mu \mathrm{g} / \mathrm{L}$ & 15 & 1.1 & 2.198 & 1.55 & 2.1 & 2.58 & 5.21 \\
\hline $\mathrm{T} 2$ & 06305700 & Selenium, dissolved, in $\mu \mathrm{g} / \mathrm{L}$ & 17 & 0.2 & 0.49 & 0.325 & 0.42 & 0.55 & 1.3 \\
\hline $\mathrm{T} 2$ & 06305700 & Selenium, total, in $\mu \mathrm{g} / \mathrm{L}$ & 17 & 0.19 & 0.556 & 0.355 & 0.41 & 0.75 & 1.6 \\
\hline T2 & 06305700 & Strontium, dissolved, in $\mu \mathrm{g} / \mathrm{L}$ & 14 & 228 & 444.4 & 429.25 & 455 & 504.25 & 537 \\
\hline $\mathrm{T} 2$ & 06305700 & Zinc, dissolved, in $\mu \mathrm{g} / \mathrm{L}$ & 14 & 1.9 & 3.100 & 2.45 & 2.9 & 3.925 & 4.8 \\
\hline T2 & 06305700 & Zinc, total, in $\mu \mathrm{g} / \mathrm{L}$ & 15 & 3 & 4.933 & 3 & 4 & 5 & 17 \\
\hline $\mathrm{T} 2$ & 06305700 & Suspended sediment, $<0.0625 \mathrm{~mm}$, percent & 31 & 36 & 76.94 & 64 & 82 & 88 & 97 \\
\hline $\mathrm{T} 2$ & 06305700 & Suspended sediment, in mg/L & 31 & 2 & 25.58 & 7 & 17 & 27 & 216 \\
\hline $\mathrm{T} 2$ & 06305700 & Suspended sediment, tons/day & 31 & 0.18 & 36.92 & 0.83 & 1.9 & 8.4 & 787 \\
\hline $\mathrm{T} 3$ & 450137106595101 & Streamflow, in cubic feet per second & 4 & 0.36 & -- & -- & -- & -- & 15 \\
\hline $\mathrm{T} 3$ & 450137106595101 & Turbidity, nephelometric turbidity ratio units & 4 & 42 & -- & -- & -- & -- & 85 \\
\hline $\mathrm{T} 3$ & 450137106595101 & Dissolved oxygen, in mg/L & 4 & 7.2 & -- & -- & -- & -- & 8 \\
\hline $\mathrm{T} 3$ & 450137106595101 & Dissolved oxygen, percent saturation & 3 & 92 & -- & -- & -- & -- & 96 \\
\hline $\mathrm{T} 3$ & 450137106595101 & $\mathrm{pH}$, in standard units & 4 & 7.5 & -- & -- & -- & -- & 8.4 \\
\hline $\mathrm{T} 3$ & 450137106595101 & Specific conductance, at 25 degrees Celsius, in $\mu \mathrm{S} / \mathrm{cm}$ & 4 & 615 & -- & -- & -- & -- & 704 \\
\hline $\mathrm{T} 3$ & 450137106595101 & Temperature, water deg C & 4 & 18 & -- & -- & -- & -- & 22 \\
\hline $\mathrm{T} 3$ & 450137106595101 & Hardness, in $\mathrm{mg} / \mathrm{L}$ as $\mathrm{CaCO}_{3}$ & 4 & 290 & -- & -- & -- & -- & 370 \\
\hline T3 & 450137106595101 & Calcium, dissolved, in $\mathrm{mg} / \mathrm{L}$ & 4 & 55.1 & -- & -- & -- & -- & 67.8 \\
\hline
\end{tabular}


Appendix. Summary statistics for water-quality constituents, Powder River structural basin, Wyoming and Montana, water years 2005-08.-Continued

[--, value cannot be calculated; $\mathrm{mg} / \mathrm{L}$, milligrams per liter; $\mu \mathrm{S} / \mathrm{cm}$, microsiemens per centimeter at 25 degrees Celsius; deg $\mathrm{C}$, degrees Celsius; $\mathrm{CaCO}_{3}$, calcium carbonate; as $\mathrm{N}$, as nitrogen; as $\mathrm{P}$, as phosphorus; $\mu \mathrm{g} / \mathrm{L}$, micrograms per liter; ng/L, nanograms per liter; mm, millimeters; *, constituent statistics were estimated using a log-probability regression; **, too many non-detected constituent concentrations to estimate statistics with log-probablity regression]

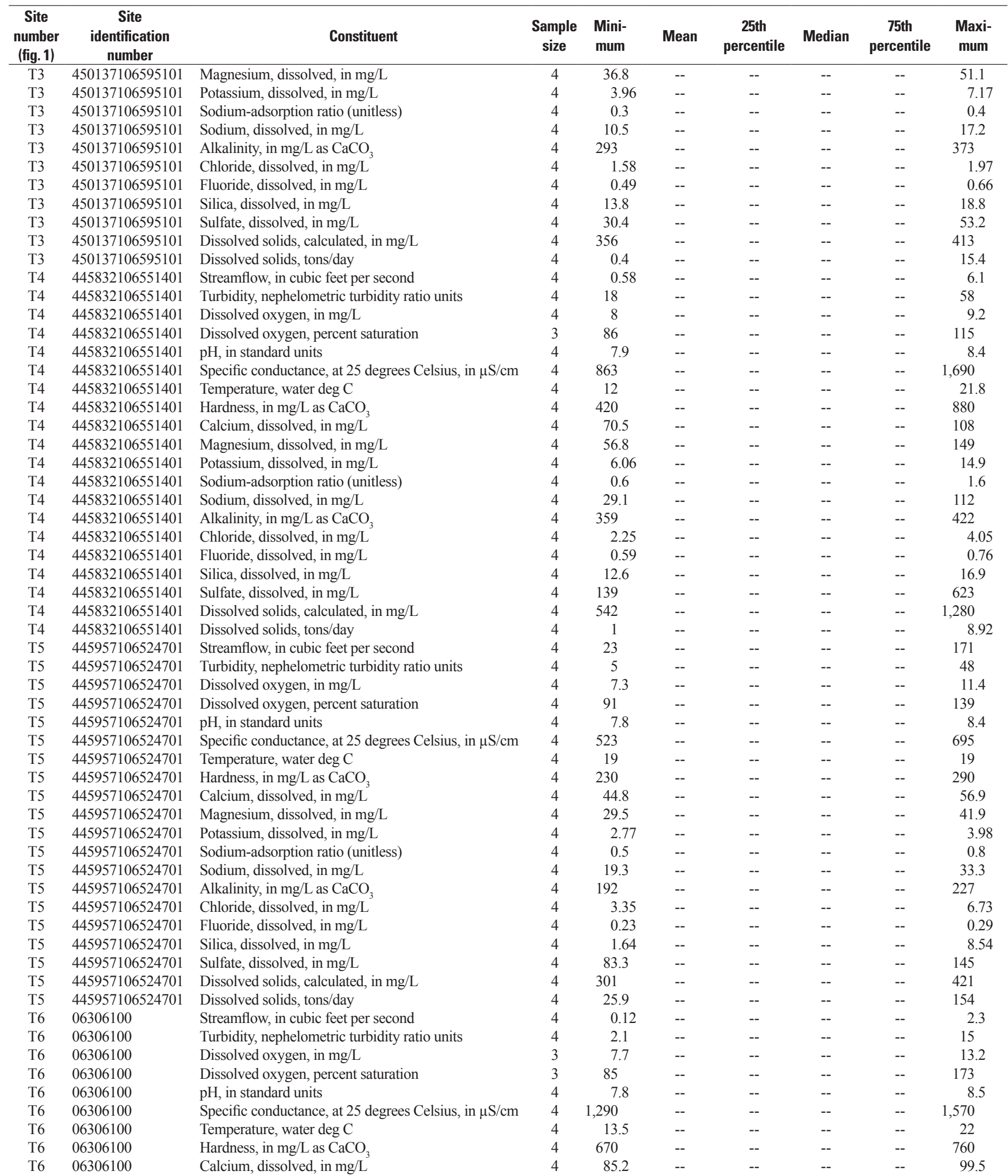


Appendix. Summary statistics for water-quality constituents, Powder River structural basin, Wyoming and Montana, water years 2005-08.-Continued

[--, value cannot be calculated; $\mathrm{mg} / \mathrm{L}$, milligrams per liter; $\mu \mathrm{S} / \mathrm{cm}$, microsiemens per centimeter at 25 degrees Celsius; deg $\mathrm{C}$, degrees $\mathrm{Celsius}^{\mathrm{C}} \mathrm{CaCO}_{3}$, calcium carbonate; as $\mathrm{N}$, as nitrogen; as $\mathrm{P}$, as phosphorus; $\mu \mathrm{g} / \mathrm{L}$, micrograms per liter; $\mathrm{ng} / \mathrm{L}$, nanograms per liter; mm, millimeters; *, constituent statistics were estimated using a log-probability regression; **, too many non-detected constituent concentrations to estimate statistics with log-probablity regression]

\begin{tabular}{|c|c|c|c|c|c|c|c|c|c|}
\hline $\begin{array}{c}\text { Site } \\
\text { number } \\
\text { (fig. 1) }\end{array}$ & $\begin{array}{c}\text { Site } \\
\begin{array}{c}\text { identification } \\
\text { number }\end{array} \\
\end{array}$ & Constituent & $\begin{array}{l}\text { Sample } \\
\text { size }\end{array}$ & $\begin{array}{l}\text { Mini- } \\
\text { mum }\end{array}$ & Mean & $\begin{array}{c}\text { 25th } \\
\text { percentile }\end{array}$ & Median & $\begin{array}{c}\text { 75th } \\
\text { percentile }\end{array}$ & $\begin{array}{l}\text { Maxi- } \\
\text { mum }\end{array}$ \\
\hline T6 & 06306100 & Magnesium, dissolved, in $\mathrm{mg} / \mathrm{L}$ & 4 & 110 & -- & -- & -- & -- & 124 \\
\hline T6 & 06306100 & Potassium, dissolved, in $\mathrm{mg} / \mathrm{L}$ & 4 & 7.88 & -- & -- & -- & -- & 9.54 \\
\hline T6 & 06306100 & Sodium, dissolved, in $\mathrm{mg} / \mathrm{L}$ & 4 & 50 & -- & -- & -- & -- & 77.8 \\
\hline T6 & 06306100 & Alkalinity, in $\mathrm{mg} / \mathrm{L}$ as $\mathrm{CaCO}_{3}$ & 4 & 408 & -- & -- & -- & -- & 514 \\
\hline T6 & 06306100 & Chloride, dissolved, in $\mathrm{mg} / \mathrm{L}^{3}$ & 4 & 2.68 & -- & -- & -- & -- & 3.49 \\
\hline $\mathrm{T} 6$ & 06306100 & Sulfate, dissolved, in $\mathrm{mg} / \mathrm{L}$ & 4 & 327 & -- & -- & -- & -- & 409 \\
\hline T6 & 06306100 & Dissolved solids, calculated, in $\mathrm{mg} / \mathrm{L}$ & 4 & 842 & -- & -- & -- & -- & 1,030 \\
\hline T6 & 06306100 & Dissolved solids, tons/day & 4 & 0.33 & -- & -- & -- & -- & 5.25 \\
\hline $\mathrm{T} 7$ & 450047106514201 & Streamflow, in cubic feet per second & 3 & 0.17 & -- & -- & -- & -- & 1.8 \\
\hline $\mathrm{T} 7$ & 450047106514201 & Turbidity, nephelometric turbidity ratio units & 3 & 1.2 & -- & -- & -- & -- & 14 \\
\hline $\mathrm{T} 7$ & 450047106514201 & Dissolved oxygen, in mg/L & 2 & 7.7 & -- & -- & -- & -- & 9.7 \\
\hline $\mathrm{T} 7$ & 450047106514201 & Calcium, dissolved, in $\mathrm{mg} / \mathrm{L}^{3}$ & 3 & 86.4 & -- & -- & -- & -- & 153 \\
\hline $\mathrm{T} 7$ & 450047106514201 & Magnesium, dissolved, in $\mathrm{mg} / \mathrm{L}$ & 3 & 146 & -- & -- & -- & -- & 463 \\
\hline $\mathrm{T} 7$ & 450047106514201 & Potassium, dissolved, in $\mathrm{mg} / \mathrm{L}$ & 3 & 9.23 & -- & -- & -- & -- & 20 \\
\hline $\mathrm{T} 7$ & 450047106514201 & Sodium-adsorption ratio (unitless) & 3 & 1.7 & -- & -- & -- & -- & 7.6 \\
\hline $\mathrm{T} 7$ & 450047106514201 & Sodium, dissolved, in $\mathrm{mg} / \mathrm{L}$ & 3 & 113 & -- & -- & -- & -- & 835 \\
\hline $\mathrm{T} 7$ & 450047106514201 & Alkalinity, in $\mathrm{mg} / \mathrm{L}$ as $\mathrm{CaCO}_{3}$ & 3 & 485 & -- & -- & -- & -- & 590 \\
\hline $\mathrm{T} 7$ & 450047106514201 & Chloride, dissolved, in $\mathrm{mg} / \mathrm{L}$ & 3 & 3.64 & -- & -- & -- & -- & 12.7 \\
\hline $\mathrm{T} 7$ & 450047106514201 & Fluoride, dissolved, in mg/L & 3 & 0.52 & -- & -- & -- & -- & 0.73 \\
\hline $\mathrm{T} 7$ & 450047106514201 & Silica, dissolved, in $\mathrm{mg} / \mathrm{L}$ & 3 & 4.21 & -- & -- & -- & -- & 9.29 \\
\hline $\mathrm{T} 7$ & 450047106514201 & Sulfate, dissolved, in $\mathrm{mg} / \mathrm{L}$ & 3 & 587 & -- & -- & -- & -- & 3,330 \\
\hline $\mathrm{T} 7$ & 450047106514201 & Dissolved solids, calculated, in $\mathrm{mg} / \mathrm{L}$ & 31 & 1,250 & -- & -- & -- & -- & 5,180 \\
\hline $\mathrm{T} 7$ & 450047106514201 & Dissolved solids, tons/day & 3 & 1.76 & -- & -- & -- & -- & 6.22 \\
\hline T8 & 06306250 & Streamflow, in cubic feet per second & 55 & 0.21 & 29.22 & 13 & 21 & 38 & 127 \\
\hline $\mathrm{T} 8$ & 06306250 & Magnesium, dissolved, in $\mathrm{mg} / \mathrm{L}$ & 51 & 39.4 & 85.64 & 68.2 & 85.7 & 101 & 167 \\
\hline $\mathrm{T} 8$ & 06306250 & Potassium, dissolved, in $\mathrm{mg} / \mathrm{L}$ & 51 & 3.8 & 7.807 & 6.23 & 7.37 & 8.67 & 17.8 \\
\hline T8 & 06306250 & Sodium-adsorption ratio (unitless) & 51 & 0.6 & 1.361 & 1.1 & 1.3 & 1.5 & 2.9 \\
\hline T8 & 06306250 & Sodium, dissolved, in $\mathrm{mg} / \mathrm{L}$ & 51 & 29.7 & 82.50 & 58.9 & 74.5 & 103 & 216 \\
\hline $\mathrm{T} 8$ & 06306250 & Alkalinity, in $\mathrm{mg} / \mathrm{L}$ as $\mathrm{CaCO}_{3}$ & 51 & 177 & 312.0 & 267 & 317 & 354 & 443 \\
\hline $\mathrm{T} 8$ & 06306250 & Chloride, dissolved, in $\mathrm{mg} / \mathrm{L}^{3}$ & 51 & 2.08 & 4.773 & 3.85 & 4.71 & 5.57 & 7.97 \\
\hline $\mathrm{T} 8$ & 06306250 & Fluoride, dissolved, in mg/L & 51 & 0.2 & 0.305 & 0.27 & 0.31 & 0.34 & 0.41 \\
\hline T8 & 06306250 & Silica, dissolved, in $\mathrm{mg} / \mathrm{L}$ & 51 & 6.6 & 12.08 & 10.7 & 12.3 & 14.1 & 16 \\
\hline T8 & 06306250 & Sulfate, dissolved, in $\mathrm{mg} / \mathrm{L}$ & 51 & 213 & 506.2 & 383 & 491 & 592 & 1,160 \\
\hline $\mathrm{T} 8$ & 06306250 & Dissolved solids, calculated, in mg/L & 51 & 501 & 1,016 & 810 & 997 & 1,180 & 2,030 \\
\hline $\mathrm{T} 8$ & 06306250 & Dissolved solids, tons/day & 51 & 1.01 & 75.51 & 42.6 & 52.6 & 73.5 & 398 \\
\hline $\mathrm{T} 8$ & 06306250 & Dissolved solids, residue on evaporation, in $\mathrm{mg} / \mathrm{L}$ & 39 & 526 & 1,072 & 853 & 1,080 & 1,250 & 2,160 \\
\hline $\mathrm{T} 8$ & 06306250 & *Ammonia, dissolved, in $\mathrm{mg} / \mathrm{L}$ as $\mathrm{N}$ & 17 & -- & 0.026 & 0.008 & 0.014 & 0.029 & 0.106 \\
\hline $\mathrm{T} 8$ & 06306250 & *Nitrate plus nitrite, dissolved, in $\mathrm{mg} / \mathrm{L}$ as $\mathrm{N}$ & 17 & -- & 0.268 & 0.063 & 0.24 & 0.431 & 0.729 \\
\hline $\mathrm{T} 8$ & 06306250 & *Nitrite, dissolved, in $\mathrm{mg} / \mathrm{L}$ as $\mathrm{N}$ & 17 & -- & 0.002 & 0.001 & 0.002 & 0.003 & 0.005 \\
\hline $\mathrm{T} 8$ & 06306250 & *Orthophosphate, dissolved, in $\mathrm{mg} / \mathrm{L}$ as $\mathrm{P}$ & 17 & -- & 0.013 & 0.005 & 0.012 & 0.021 & 0.028 \\
\hline $\mathrm{T} 8$ & 06306250 & Phosphorus, total, in $\mathrm{mg} / \mathrm{L}$ & 17 & 0.013 & 0.127 & 0.027 & 0.058 & 0.236 & 0.34 \\
\hline $\mathrm{T} 8$ & 06306250 & Total nitrogen, total, in $\mathrm{mg} / \mathrm{L}$ & 17 & 0.29 & 0.821 & 0.49 & 0.83 & 1 & 1.73 \\
\hline $\mathrm{T} 8$ & 06306250 & *Aluminum, dissolved, in $\mu \mathrm{g} / \mathrm{L}$ & 17 & -- & 1.543 & 1.054 & 1.36 & 1.85 & 3.1 \\
\hline T8 & 06306250 & Aluminum, total, in $\mu \mathrm{g} / \mathrm{L}$ & 36 & 35 & 1,084 & 125.25 & 304.5 & 1,550 & 9,480 \\
\hline
\end{tabular}


Appendix. Summary statistics for water-quality constituents, Powder River structural basin, Wyoming and Montana, water years 2005-08.-Continued

[--, value cannot be calculated; $\mathrm{mg} / \mathrm{L}$, milligrams per liter; $\mu \mathrm{S} / \mathrm{cm}$, microsiemens per centimeter at 25 degrees Celsius; deg $\mathrm{C}$, degrees Celsius; $\mathrm{CaCO}_{3}$, calcium carbonate; as $\mathrm{N}$, as nitrogen; as $\mathrm{P}$, as phosphorus; $\mu \mathrm{g} / \mathrm{L}$, micrograms per liter; ng/L, nanograms per liter; mm, millimeters; *, constituent statistics were estimated using a log-probability regression; **, too many non-detected constituent concentrations to estimate statistics with log-probablity regression]

\begin{tabular}{|c|c|c|c|c|c|c|c|c|c|}
\hline $\begin{array}{c}\text { Site } \\
\text { number } \\
\text { (fig. 1) } \\
\end{array}$ & $\begin{array}{c}\text { Site } \\
\begin{array}{c}\text { identification } \\
\text { number }\end{array} \\
\end{array}$ & Constituent & $\begin{array}{l}\text { Sample } \\
\text { size }\end{array}$ & $\begin{array}{l}\text { Mini- } \\
\text { mum }\end{array}$ & Mean & $\begin{array}{c}\text { 25th } \\
\text { percentile }\end{array}$ & Median & $\begin{array}{c}\text { 75th } \\
\text { percentile }\end{array}$ & $\begin{array}{l}\text { Maxi- } \\
\text { mum }\end{array}$ \\
\hline T8 & 06306250 & Arsenic, dissolved, in $\mu \mathrm{g} / \mathrm{L}$ & 36 & 0.31 & 0.72 & 0.543 & 0.75 & 0.9 & 1.1 \\
\hline $\mathrm{T} 8$ & 06306250 & *Arsenic, total, in $\mu \mathrm{g} / \mathrm{L}$ & 34 & -- & 1.629 & 0.98 & 1.4 & 2 & 6.1 \\
\hline $\mathrm{T} 8$ & 06306250 & Barium, total, in $\mu \mathrm{g} / \mathrm{L}$ & 36 & 37.2 & 58.84 & 45 & 50.35 & 63.375 & 210 \\
\hline T8 & 06306250 & **Beryllium, dissolved, in $\mu \mathrm{g} / \mathrm{L}$ & 17 & -- & -- & -- & -- & -- & 0.12 \\
\hline $\mathrm{T} 8$ & 06306250 & ${ }^{*}$ Beryllium, total, in $\mu \mathrm{g} / \mathrm{L}$ & 36 & -- & 0.1 & 0.02 & 0.046 & 0.13 & 0.84 \\
\hline $\mathrm{T} 8$ & 06306250 & ${ }^{*}$ Cadmium, total, in $\mu \mathrm{g} / \mathrm{L}$ & 15 & -- & 0.056 & 0.02 & 0.033 & 0.08 & 0.18 \\
\hline $\mathrm{T} 8$ & 06306250 & Chromium, dissolved, in $\mu \mathrm{g} / \mathrm{L}$ & 1 & -- & -- & -- & -- & -- & 0.22 \\
\hline T8 & 06306250 & ${ }^{*}$ Chromium, total, in $\mu \mathrm{g} / \mathrm{L}$ & 15 & -- & 3.270 & 2 & 2.7 & 4 & 9 \\
\hline $\mathrm{T} 8$ & 06306250 & Copper, dissolved, in $\mu \mathrm{g} / \mathrm{L}$ & 15 & 0.57 & 2.757 & 1.9 & 2.7 & 3.4 & 5.1 \\
\hline $\mathrm{T} 8$ & 06306250 & Copper, total, in $\mu \mathrm{g} / \mathrm{L}$ & 15 & 2.5 & 6.293 & 4 & 6 & 8.7 & 14.6 \\
\hline T8 & 06306250 & *Iron, dissolved, in $\mu \mathrm{g} / \mathrm{L}$ & 36 & -- & 14.26 & 6 & 7.5 & 11.75 & 100 \\
\hline $\mathrm{T} 8$ & 06306250 & Manganese, total, in $\mu \mathrm{g} / \mathrm{L}$ & 17 & 58.3 & 241.2 & 88.05 & 168 & 421 & 537 \\
\hline T8 & 06306250 & Mercury, total, ng/L & 5 & 0.92 & -- & -- & -- & -- & 9.76 \\
\hline T8 & 06306250 & $* *$ Mercury, total, $\mu \mathrm{g} / \mathrm{L}$ & 3 & -- & -- & -- & -- & -- & $<0.01$ \\
\hline T8 & 06306250 & Nickel, dissolved, in $\mu \mathrm{g} / \mathrm{L}$ & 15 & 1 & 2.960 & 1.46 & 3.17 & 3.76 & 5.56 \\
\hline $\mathrm{T} 8$ & 06306250 & Nickel, total, in $\mu \mathrm{g} / \mathrm{L}$ & 15 & 2.7 & 5.369 & 4 & 4.8 & 6.76 & 10.9 \\
\hline $\mathrm{T} 8$ & 06306250 & Selenium, dissolved, in $\mu \mathrm{g} / \mathrm{L}$ & 17 & 0.23 & 0.874 & 0.63 & 0.78 & 1.15 & 1.7 \\
\hline T8 & 06306250 & Selenium, total, in $\mu \mathrm{g} / \mathrm{L}$ & 36 & 0.12 & 0.92 & 0.632 & 0.85 & 1.2 & 2 \\
\hline $\mathrm{T} 8$ & 06306250 & Strontium, dissolved, in $\mu \mathrm{g} / \mathrm{L}$ & 14 & 920 & 1,658 & 1,296 & 1,770 & 1,943 & 2,520 \\
\hline T8 & 06306250 & $*$ Zinc, dissolved, in $\mu \mathrm{g} / \mathrm{L}$ & 15 & -- & 3.065 & 1.2 & 2 & 2.5 & 15.9 \\
\hline $\mathrm{T} 8$ & 06306250 & Zinc, total, in $\mu \mathrm{g} / \mathrm{L}$ & 15 & 2 & 9.140 & 3 & 8 & 12 & 27 \\
\hline $\mathrm{T} 8$ & 06306250 & Suspended sediment, $<0.0625 \mathrm{~mm}$, percent & 34 & 43 & 82.03 & 76.75 & 87 & 92 & 97 \\
\hline T8 & 06306250 & Suspended sediment, in $\mathrm{mg} / \mathrm{L}$ & 34 & 21 & 122.4 & 60 & 84 & 174 & 431 \\
\hline T8 & 06306250 & Suspended sediment, tons/day & 34 & 0.02 & 12.60 & 1.7 & 4.1 & 14.5 & 119 \\
\hline T9 & 06306300 & Calcium, dissolved, in $\mathrm{mg} / \mathrm{L}^{3}$ & 64 & 20.1 & 53.02 & 44.475 & 55.5 & 63.05 & 78.9 \\
\hline T9 & 06306300 & Magnesium, dissolved, in $\mathrm{mg} / \mathrm{L}$ & 64 & 7.83 & 31.23 & 23.025 & 34.2 & 38.475 & 50 \\
\hline T9 & 06306300 & Potassium, dissolved, in $\mathrm{mg} / \mathrm{L}$ & 64 & 1 & 2.750 & 1.995 & 2.805 & 3.1 & 7.34 \\
\hline T9 & 06306300 & Sodium-adsorption ratio (unitless) & 64 & 0.2 & 0.719 & 0.5 & 0.7 & 0.875 & 1.5 \\
\hline T9 & 06306300 & Sodium, dissolved, in $\mathrm{mg} / \mathrm{L}$ & 64 & 4.97 & 27.72 & 17.825 & 28.25 & 35.45 & 65.2 \\
\hline T9 & 06306300 & Acid neutralizing capacity, in $\mathrm{mg} / \mathrm{L}$ as $\mathrm{CaCO}_{3}$ & 42 & 81 & 200.2 & 171.25 & 212 & 235.25 & 273 \\
\hline T9 & 06306300 & Alkalinity, in $\mathrm{mg} / \mathrm{L}$ as $\mathrm{CaCO}_{3}$ & 64 & 73 & 193.8 & 161.25 & 206 & 235.75 & 276 \\
\hline T9 & 06306300 & Chloride, dissolved, in $\mathrm{mg} / \mathrm{L}^{3}$ & 64 & 0.83 & 3.923 & 2.67 & 3.83 & 4.832 & 7.93 \\
\hline T9 & 06306300 & Fluoride, dissolved, in $\mathrm{mg} / \mathrm{L}$ & 63 & 0.08 & 0.273 & 0.2 & 0.29 & 0.32 & 0.61 \\
\hline T9 & 06306300 & Silica, dissolved, in $\mathrm{mg} / \mathrm{L}$ & 63 & 2.37 & 5.852 & 3.97 & 5.77 & 7.63 & 10.3 \\
\hline T9 & 06306300 & Sulfate, dissolved, in $\mathrm{mg} / \mathrm{L}$ & 64 & 20.5 & 117.4 & 79.175 & 124.5 & 149.25 & 237 \\
\hline T9 & 06306300 & Dissolved solids, calculated, in mg/L & 60 & 108 & 364.3 & 270.75 & 385.5 & 451.5 & 571 \\
\hline T9 & 06306300 & Dissolved solids, tons/day & 62 & 26.2 & 357.0 & 160.5 & 188.5 & 333.5 & 3,580 \\
\hline T9 & 06306300 & Dissolved solids, residue on evaporation, in $\mathrm{mg} / \mathrm{L}$ & 43 & 118 & 381.1 & 281 & 411 & 466 & 561 \\
\hline T9 & 06306300 & *Ammonia, dissolved, in $\mathrm{mg} / \mathrm{L}$ as $\mathrm{N}$ & 20 & -- & 0.02 & 0.006 & 0.012 & 0.025 & 0.083 \\
\hline T9 & 06306300 & *Nitrate plus nitrite, dissolved, in $\mathrm{mg} / \mathrm{L}$ as $\mathrm{N}$ & 20 & -- & 0.071 & 0.009 & 0.03 & 0.07 & 0.434 \\
\hline T9 & 06306300 & *Nitrite, dissolved, in $\mathrm{mg} / \mathrm{L}$ as $\mathrm{N}$ & 20 & -- & 0.002 & 0.001 & 0.001 & 0.003 & 0.009 \\
\hline T9 & 06306300 & ${ }^{*}$ Orthophosphate, dissolved, in $\mathrm{mg} / \mathrm{L}$ as $\mathrm{P}$ & 20 & -- & 0.011 & 0.003 & 0.007 & 0.013 & 0.057 \\
\hline T9 & 06306300 & Phosphorus, total, in mg/L & 20 & 0.019 & 0.084 & 0.029 & 0.041 & 0.08 & 0.53 \\
\hline T9 & 06306300 & Total nitrogen, total, in $\mathrm{mg} / \mathrm{L}$ & 20 & 0.22 & 0.483 & 0.282 & 0.355 & 0.425 & 1.99 \\
\hline
\end{tabular}


Appendix. Summary statistics for water-quality constituents, Powder River structural basin, Wyoming and Montana, water years 2005-08.-Continued

[--, value cannot be calculated; $\mathrm{mg} / \mathrm{L}$, milligrams per liter; $\mu \mathrm{S} / \mathrm{cm}$, microsiemens per centimeter at 25 degrees Celsius; deg $\mathrm{C}$, degrees $\mathrm{Celsius}^{\mathrm{C}} \mathrm{CaCO}_{3}$, calcium carbonate; as $\mathrm{N}$, as nitrogen; as $\mathrm{P}$, as phosphorus; $\mu \mathrm{g} / \mathrm{L}$, micrograms per liter; $\mathrm{ng} / \mathrm{L}$, nanograms per liter; mm, millimeters; *, constituent statistics were estimated using a log-probability regression; **, too many non-detected constituent concentrations to estimate statistics with log-probablity regression]

\begin{tabular}{|c|c|c|c|c|c|c|c|c|c|}
\hline $\begin{array}{c}\text { Site } \\
\text { number } \\
\text { (fig. 1) }\end{array}$ & $\begin{array}{c}\text { Site } \\
\text { identification } \\
\text { number } \\
\end{array}$ & Constituent & $\begin{array}{l}\text { Sample } \\
\text { size }\end{array}$ & $\begin{array}{l}\text { Mini- } \\
\text { mum }\end{array}$ & Mean & $\begin{array}{c}\text { 25th } \\
\text { percentile }\end{array}$ & Median & $\begin{array}{c}\text { 75th } \\
\text { percentile }\end{array}$ & $\begin{array}{c}\text { Maxi- } \\
\text { mum }\end{array}$ \\
\hline T9 & 06306300 & *Aluminum, dissolved, in $\mu \mathrm{g} / \mathrm{L}$ & 20 & -- & 4.096 & 1.1 & 1.7 & 4.175 & 25.1 \\
\hline T9 & 06306300 & Aluminum, total, in $\mu \mathrm{g} / \mathrm{L}$ & 29 & 18 & 536.3 & 70.5 & 140 & 363.5 & 5,810 \\
\hline T9 & 06306300 & *Arsenic, total, in $\mu \mathrm{g} / \mathrm{L}$ & 29 & -- & 0.88 & 0.59 & 0.75 & 0.915 & 3 \\
\hline T9 & 06306300 & Barium, dissolved, in $\mu \mathrm{g} / \mathrm{L}$ & 20 & 24 & 47.15 & 41 & 48.5 & 55.5 & 67 \\
\hline T9 & 06306300 & Barium, total, in $\mu \mathrm{g} / \mathrm{L}$ & 29 & 35 & 59.01 & 47.5 & 55.3 & 60.3 & 155 \\
\hline T9 & 06306300 & Boron, dissolved, in $\mu \mathrm{g} / \mathrm{L}$ & 15 & 35 & 64.27 & 54 & 62 & 69 & 99 \\
\hline T9 & 06306300 & Boron, total, in $\mu \mathrm{g} / \mathrm{L}$ & 3 & 61 & -- & -- & -- & -- & 79 \\
\hline T9 & 06306300 & **Cadmium, dissolved, in $\mu \mathrm{g} / \mathrm{L}$ & 20 & -- & -- & -- & -- & -- & 0.05 \\
\hline T9 & 06306300 & *Cadmium, total, in $\mu \mathrm{g} / \mathrm{L}$ & 24 & -- & 0.034 & 0.006 & 0.015 & 0.022 & 0.35 \\
\hline T9 & 06306300 & Chromium, dissolved, in $\mu \mathrm{g} / \mathrm{L}$ & 6 & 0.06 & 0.097 & 0.068 & 0.085 & 0.12 & 0.18 \\
\hline T9 & 06306300 & *Chromium, total, in $\mu \mathrm{g} / \mathrm{L}$ & 24 & -- & 1.377 & 0.407 & 0.965 & 2 & 7 \\
\hline T9 & 06306300 & Lead, total, in $\mu \mathrm{g} / \mathrm{L}$ & 24 & 0.06 & 1.090 & 0.132 & 0.26 & 0.73 & 10.8 \\
\hline T9 & 06306300 & Lithium, dissolved, in $\mu \mathrm{g} / \mathrm{L}$ & 14 & 10 & 19.28 & 15.475 & 19.1 & 22.2 & 36.6 \\
\hline T9 & 06306300 & Manganese, dissolved, in $\mu \mathrm{g} / \mathrm{L}$ & 30 & 0.5 & 15.72 & 7.15 & 11.15 & 24.65 & 52.6 \\
\hline T9 & 06306300 & Manganese, total, in $\mu \mathrm{g} / \mathrm{L}$ & 20 & 13.7 & 61.66 & 28.7 & 50.25 & 59.675 & 290 \\
\hline T9 & 06306300 & Mercury, total, ng/L & 5 & 0.59 & -- & -- & -- & -- & 2.35 \\
\hline T9 & 06306300 & $* *$ Mercury, total, $\mu \mathrm{g} / \mathrm{L}$ & 4 & -- & -- & -- & -- & -- & 0.01 \\
\hline T9 & 06306300 & Nickel, dissolved, in $\mu \mathrm{g} / \mathrm{L}$ & 21 & 0.22 & 1.493 & 0.67 & 1.5 & 2.09 & 3.06 \\
\hline T9 & 06306300 & Nickel, total, in $\mu \mathrm{g} / \mathrm{L}$ & 24 & 0.63 & 2.400 & 1.2 & 1.82 & 2.395 & 12.1 \\
\hline T9 & 06306300 & Selenium, dissolved, in $\mu \mathrm{g} / \mathrm{L}$ & 20 & 0.15 & 0.384 & 0.222 & 0.3 & 0.5 & 1 \\
\hline T9 & 06306300 & Selenium, total, in $\mu \mathrm{g} / \mathrm{L}$ & 29 & 0.09 & 0.388 & 0.22 & 0.31 & 0.395 & 1.6 \\
\hline T9 & 06306300 & Strontium, dissolved, in $\mu \mathrm{g} / \mathrm{L}$ & 15 & 219 & 470.2 & 443 & 508 & 532 & 567 \\
\hline T9 & 06306300 & Zinc, dissolved, in $\mu \mathrm{g} / \mathrm{L}$ & 18 & 0.81 & 1.928 & 1.275 & 1.7 & 2.3 & 4.8 \\
\hline T9 & 06306300 & $*$ Zinc, total, in $\mu \mathrm{g} / \mathrm{L}$ & 24 & -- & 5.739 & 2 & 2.2 & 4.875 & 44 \\
\hline $\mathrm{T} 10$ & 451607106372801 & Specific conductance, at 25 degrees Celsius, in $\mu \mathrm{S} / \mathrm{cm}$ & 4 & 363 & -- & -- & -- & -- & 540 \\
\hline $\mathrm{T} 10$ & 451607106372801 & Temperature, water deg C & 4 & 15 & -- & -- & -- & -- & 20 \\
\hline $\mathrm{T} 10$ & 451607106372801 & Hardness, in $\mathrm{mg} / \mathrm{L}$ as $\mathrm{CaCO}_{3}$ & 4 & 160 & -- & -- & -- & -- & 220 \\
\hline $\mathrm{T} 10$ & 451607106372801 & Calcium, dissolved, in $\mathrm{mg} / \mathrm{L}^{3}$ & 4 & 36.2 & -- & -- & -- & -- & 48.6 \\
\hline $\mathrm{T} 10$ & 451607106372801 & Magnesium, dissolved, in mg/L & 4 & 17 & -- & -- & -- & -- & 28.8 \\
\hline $\mathrm{T} 10$ & 451607106372801 & Potassium, dissolved, in $\mathrm{mg} / \mathrm{L}$ & 4 & 1.92 & -- & -- & -- & -- & 3.62 \\
\hline $\mathrm{T} 10$ & 451607106372801 & Sodium-adsorption ratio (unitless) & 4 & 0.5 & -- & -- & -- & -- & 1.1 \\
\hline $\mathrm{T} 10$ & 451607106372801 & Sodium, dissolved, in $\mathrm{mg} / \mathrm{L}$ & 4 & 14.7 & -- & -- & -- & -- & 36.5 \\
\hline $\mathrm{T} 10$ & 451607106372801 & Alkalinity, in $\mathrm{mg} / \mathrm{L}$ as $\mathrm{CaCO}_{3}$ & 4 & 130 & -- & -- & -- & -- & 174 \\
\hline $\mathrm{T} 10$ & 451607106372801 & Chloride, dissolved, in $\mathrm{mg} / \mathrm{L}$ & 4 & 1.46 & -- & -- & -- & -- & 3.4 \\
\hline $\mathrm{T} 10$ & 451607106372801 & Fluoride, dissolved, in mg/L & 4 & 0.18 & -- & -- & -- & -- & 0.3 \\
\hline $\mathrm{T} 10$ & 451607106372801 & Silica, dissolved, in $\mathrm{mg} / \mathrm{L}$ & 4 & 0.67 & -- & -- & -- & -- & 5.47 \\
\hline $\mathrm{T} 10$ & 451607106372801 & Sulfate, dissolved, in $\mathrm{mg} / \mathrm{L}$ & 4 & 54.4 & -- & -- & -- & -- & 111 \\
\hline $\mathrm{T} 10$ & 451607106372801 & Dissolved solids, calculated, in $\mathrm{mg} / \mathrm{L}$ & 4 & 209 & -- & -- & -- & -- & 322 \\
\hline $\mathrm{T} 10$ & 451607106372801 & Dissolved solids, tons/day & 4 & 132 & -- & -- & -- & -- & 276 \\
\hline T11 & 06307570 & Streamflow, in cubic feet per second & 3 & 0 & -- & -- & -- & -- & 0.12 \\
\hline T11 & 06307570 & Turbidity, nephelometric turbidity ratio units & 3 & 3.9 & -- & -- & -- & -- & 14 \\
\hline T11 & 06307570 & Dissolved oxygen, in $\mathrm{mg} / \mathrm{L}$ & 1 & -- & -- & -- & -- & -- & 10 \\
\hline T11 & 06307570 & Dissolved oxygen, percent saturation & 1 & -- & -- & -- & -- & -- & 123 \\
\hline T11 & 06307570 & $\mathrm{pH}$, in standard units & 3 & 7.9 & -- & -- & -- & -- & 9.3 \\
\hline
\end{tabular}


Appendix. Summary statistics for water-quality constituents, Powder River structural basin, Wyoming and Montana, water years 2005-08.-Continued

[--, value cannot be calculated; $\mathrm{mg} / \mathrm{L}$, milligrams per liter; $\mu \mathrm{S} / \mathrm{cm}$, microsiemens per centimeter at 25 degrees Celsius; deg C, degrees Celsius; $\mathrm{CaCO}_{3}$, calcium carbonate; as $\mathrm{N}$, as nitrogen; as $\mathrm{P}$, as phosphorus; $\mu \mathrm{g} / \mathrm{L}$, micrograms per liter; $\mathrm{ng} / \mathrm{L}$, nanograms per liter; mm, millimeters; *, constituent statistics were estimated using a log-probability regression; **, too many non-detected constituent concentrations to estimate statistics with log-probablity regression]

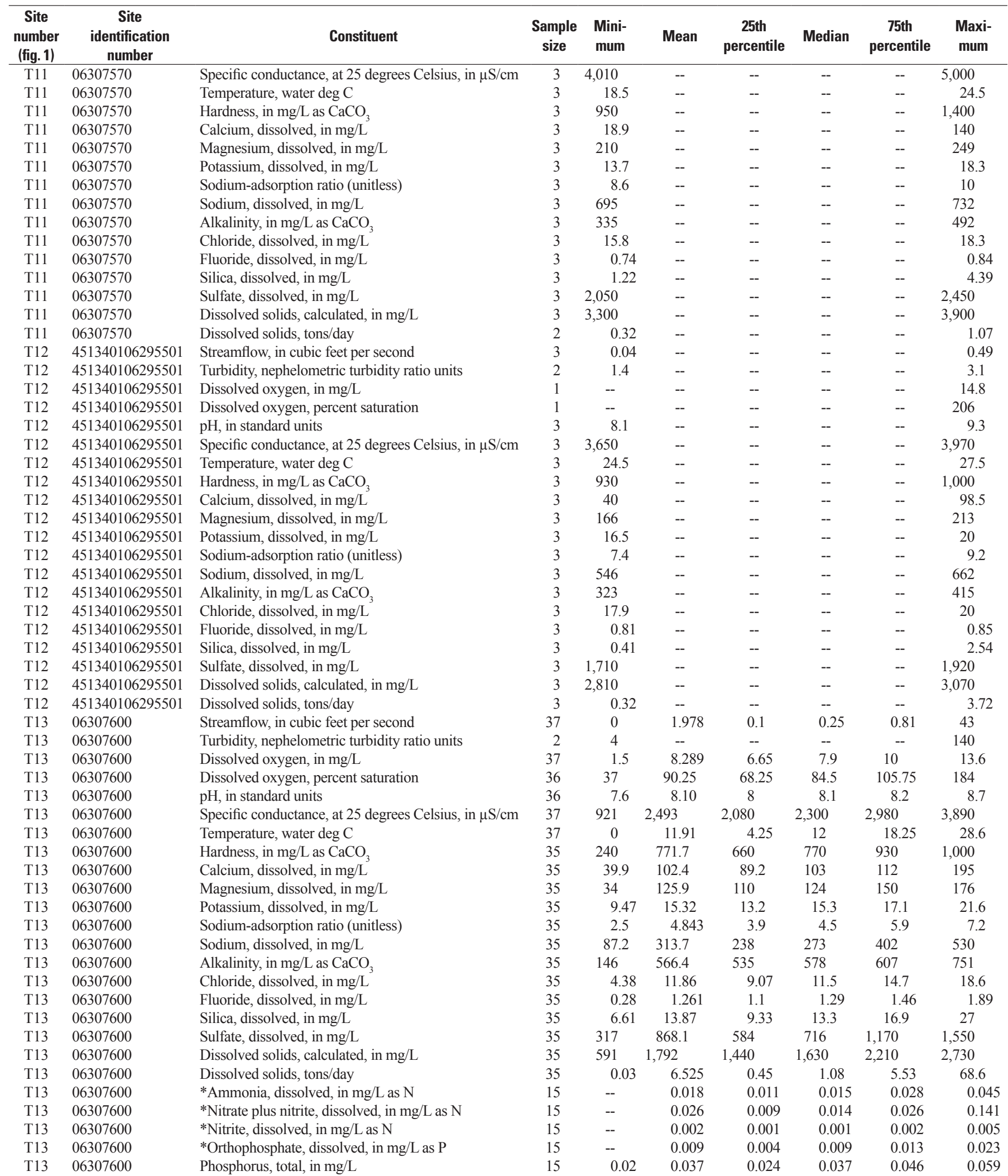


Appendix. Summary statistics for water-quality constituents, Powder River structural basin, Wyoming and Montana, water years 2005-08.-Continued

[--, value cannot be calculated; $\mathrm{mg} / \mathrm{L}$, milligrams per liter; $\mu \mathrm{S} / \mathrm{cm}$, microsiemens per centimeter at 25 degrees Celsius; deg $\mathrm{C}$, degrees $\mathrm{Celsius}^{\mathrm{C}} \mathrm{CaCO}_{3}$, calcium carbonate; as $\mathrm{N}$, as nitrogen; as $\mathrm{P}$, as phosphorus; $\mu \mathrm{g} / \mathrm{L}$, micrograms per liter; $\mathrm{ng} / \mathrm{L}$, nanograms per liter; mm, millimeters; *, constituent statistics were estimated using a log-probability regression; **, too many non-detected constituent concentrations to estimate statistics with log-probablity regression]

\begin{tabular}{|c|c|c|c|c|c|c|c|c|c|}
\hline $\begin{array}{c}\text { Site } \\
\text { number } \\
\text { (fig. 1) }\end{array}$ & $\begin{array}{c}\text { Site } \\
\text { identification } \\
\text { number }\end{array}$ & Constituent & $\begin{array}{c}\text { Sample } \\
\text { size }\end{array}$ & $\begin{array}{l}\text { Mini- } \\
\text { mum }\end{array}$ & Mean & $\begin{array}{c}25 \text { th } \\
\text { percentile }\end{array}$ & Median & $\begin{array}{c}\text { 75th } \\
\text { percentile }\end{array}$ & $\begin{array}{l}\text { Maxi- } \\
\text { mum }\end{array}$ \\
\hline T13 & 06307600 & Total nitrogen, total, in $\mathrm{mg} / \mathrm{L}$ & 15 & 0.31 & 0.489 & 0.39 & 0.49 & 0.57 & 0.63 \\
\hline T13 & 06307600 & Arsenic, dissolved, in $\mu \mathrm{g} / \mathrm{L}$ & 14 & 0.53 & 1.074 & 0.75 & 0.985 & 1.35 & 1.9 \\
\hline T13 & 06307600 & *Arsenic, total, in $\mu \mathrm{g} / \mathrm{L}$ & 14 & -- & 1.279 & 0.812 & 1.1 & 1.492 & 2.6 \\
\hline T13 & 06307600 & Barium, dissolved, in $\mu \mathrm{g} / \mathrm{L}$ & 14 & 18 & 45.07 & 41.75 & 46 & 49 & 71 \\
\hline $\mathrm{T} 13$ & 06307600 & **Beryllium, total, in $\mu \mathrm{g} / \mathrm{L}$ & 14 & -- & -- & -- & -- & -- & $<0.12$ \\
\hline T13 & 06307600 & Boron, dissolved, in $\mu \mathrm{g} / \mathrm{L}$ & 9 & 197 & 251.4 & 235.5 & 257 & 265.5 & 294 \\
\hline T13 & 06307600 & **Cadmium, dissolved, in $\mu \mathrm{g} / \mathrm{L}$ & 12 & -- & -- & -- & -- & -- & $<0.08$ \\
\hline T13 & 06307600 & *Cadmium, total, in $\mu \mathrm{g} / \mathrm{L}$ & 12 & -- & 0.04 & 0.015 & 0.023 & 0.039 & 0.2 \\
\hline T13 & 06307600 & **Chromium, dissolved, in $\mu \mathrm{g} / \mathrm{L}$ & 3 & -- & -- & -- & -- & -- & 0.42 \\
\hline T13 & 06307600 & *Chromium, total, in $\mu \mathrm{g} / \mathrm{L}$ & 12 & -- & 2.485 & 0.678 & 2 & 3.75 & 6 \\
\hline T13 & 06307600 & Lead, total, in $\mu \mathrm{g} / \mathrm{L}$ & 12 & 0.08 & 0.148 & 0.093 & 0.13 & 0.188 & 0.28 \\
\hline T13 & 06307600 & Lithium, dissolved, in $\mu \mathrm{g} / \mathrm{L}$ & 9 & 61.4 & 95.70 & 86.45 & 100 & 104.5 & 119 \\
\hline T13 & 06307600 & Manganese, dissolved, in $\mu \mathrm{g} / \mathrm{L}$ & 14 & 45.3 & 117.9 & 75.575 & 104 & 140.5 & 228 \\
\hline T13 & 06307600 & Manganese, total, in $\mu \mathrm{g} / \mathrm{L}$ & 14 & 57.1 & 123.8 & 79.375 & 113.5 & 143.5 & 234 \\
\hline T13 & 06307600 & Mercury, total, ng/L & 4 & 0.65 & -- & -- & -- & -- & 1.06 \\
\hline T13 & 06307600 & $* *$ Mercury, total, $\mu \mathrm{g} / \mathrm{L}$ & 3 & -- & -- & -- & -- & -- & $<0.01$ \\
\hline T13 & 06307600 & Nickel, dissolved, in $\mu \mathrm{g} / \mathrm{L}$ & 12 & 1.8 & 3.303 & 2.15 & 3.345 & 4.265 & 5.03 \\
\hline T13 & 06307600 & Nickel, total, in $\mu \mathrm{g} / \mathrm{L}$ & 12 & 1.6 & 4.003 & 2.727 & 3.91 & 4.935 & 8.57 \\
\hline T13 & 06307600 & Selenium, dissolved, in $\mu \mathrm{g} / \mathrm{L}$ & 14 & 0.15 & 0.531 & 0.215 & 0.515 & 0.725 & 1.2 \\
\hline T13 & 06307600 & Selenium, total, in $\mu \mathrm{g} / \mathrm{L}$ & 14 & 0.14 & 0.551 & 0.22 & 0.36 & 0.925 & 1.2 \\
\hline $\mathrm{T} 13$ & 06307600 & Strontium, dissolved, in $\mu \mathrm{g} / \mathrm{L}$ & 9 & 1,240 & 1,404 & 1,340 & 1,430 & 1,475 & 1,540 \\
\hline $\mathrm{T} 13$ & 06307600 & $*$ Zinc, dissolved, in $\mu \mathrm{g} / \mathrm{L}$ & 12 & -- & 3.015 & 1.925 & 2.24 & 3.525 & 6.5 \\
\hline T13 & 06307600 & $*$ Zinc, total, in $\mu \mathrm{g} / \mathrm{L}$ & 12 & -- & 3.085 & 2 & 2.858 & 4 & 5 \\
\hline T14 & 06307616 & Specific conductance, at 25 degrees Celsius, in $\mu \mathrm{S} / \mathrm{cm}$ & 67 & 263 & 583.5 & 436 & 623 & 715 & 807 \\
\hline T14 & 06307616 & Temperature, water deg C & 67 & 0 & 13.94 & 8 & 14 & 20.5 & 27 \\
\hline T14 & 06307616 & Hardness, in $\mathrm{mg} / \mathrm{L}$ as $\mathrm{CaCO}_{3}$ & 63 & 110 & 244.0 & 180 & 260 & 300 & 370 \\
\hline T14 & 06307616 & Calcium, dissolved, in $\mathrm{mg} / \mathrm{L}^{3}$ & 63 & 26.6 & 47.78 & 35.9 & 49.7 & 56.4 & 69.6 \\
\hline T14 & 06307616 & Magnesium, dissolved, in $\mathrm{mg} / \mathrm{L}$ & 63 & 11 & 30.26 & 19.9 & 33.2 & 38 & 47 \\
\hline T14 & 06307616 & Potassium, dissolved, in $\mathrm{mg} / \mathrm{L}$ & 63 & 1.42 & 3.202 & 2.54 & 3.38 & 3.69 & 4.67 \\
\hline T14 & 06307616 & Sodium-adsorption ratio (unitless) & 63 & 0.4 & 0.924 & 0.6 & 1 & 1.2 & 1.4 \\
\hline T14 & 06307616 & Sodium, dissolved, in mg/L & 63 & 9.04 & 33.99 & 19.8 & 39.4 & 44.8 & 54.5 \\
\hline T14 & 06307616 & Alkalinity, in $\mathrm{mg} / \mathrm{L}$ as $\mathrm{CaCO}_{3}$ & 63 & 99 & 190.9 & 144 & 198 & 226 & 282 \\
\hline T14 & 06307616 & Chloride, dissolved, in $\mathrm{mg} / \mathrm{L}$ & 63 & 1.15 & 3.685 & 2.23 & 4.04 & 4.83 & 7.59 \\
\hline T14 & 06307616 & Fluoride, dissolved, in mg/L & 63 & 0.14 & 0.295 & 0.21 & 0.33 & 0.36 & 0.43 \\
\hline T14 & 06307616 & Silica, dissolved, in $\mathrm{mg} / \mathrm{L}$ & 63 & 0.76 & 3.558 & 1.81 & 3.1 & 5.46 & 7.82 \\
\hline T14 & 06307616 & Sulfate, dissolved, in $\mathrm{mg} / \mathrm{L}$ & 63 & 32 & 121.5 & 73.5 & 142 & 157 & 184 \\
\hline T14 & 06307616 & Dissolved solids, calculated, in $\mathrm{mg} / \mathrm{L}$ & 60 & 162 & 359.0 & 257.5 & 387 & 444 & 532 \\
\hline T14 & 06307616 & Dissolved solids, tons/day & 60 & 65.2 & 362.7 & 122.25 & 213.5 & 321.75 & 2,270 \\
\hline T14 & 06307616 & *Ammonia, dissolved, in $\mathrm{mg} / \mathrm{L}$ as $\mathrm{N}$ & 20 & -- & 0.013 & 0.005 & 0.007 & 0.012 & 0.095 \\
\hline T14 & 06307616 & *Nitrate plus nitrite, dissolved, in $\mathrm{mg} / \mathrm{L}$ as $\mathrm{N}$ & 20 & -- & 0.023 & 0.007 & 0.009 & 0.022 & 0.16 \\
\hline T14 & 06307616 & *Nitrite, dissolved, in $\mathrm{mg} / \mathrm{L}$ as $\mathrm{N}$ & 20 & -- & 0.002 & 0.001 & 0.001 & 0.002 & 0.007 \\
\hline T14 & 06307616 & *Orthophosphate, dissolved, in $\mathrm{mg} / \mathrm{L}$ as $\mathrm{P}$ & 20 & -- & 0.005 & 0.003 & 0.004 & 0.006 & 0.013 \\
\hline T14 & 06307616 & Phosphorus, total, in mg/L & 20 & 0.007 & 0.036 & 0.009 & 0.018 & 0.053 & 0.13 \\
\hline
\end{tabular}


Appendix. Summary statistics for water-quality constituents, Powder River structural basin, Wyoming and Montana, water years 2005-08.-Continued

[--, value cannot be calculated; $\mathrm{mg} / \mathrm{L}$, milligrams per liter; $\mu \mathrm{S} / \mathrm{cm}$, microsiemens per centimeter at 25 degrees Celsius; deg $\mathrm{C}$, degrees Celsius; $\mathrm{CaCO}_{3}$, calcium carbonate; as $\mathrm{N}$, as nitrogen; as $\mathrm{P}$, as phosphorus; $\mu \mathrm{g} / \mathrm{L}$, micrograms per liter; ng/L, nanograms per liter; mm, millimeters; *, constituent statistics were estimated using a log-probability regression; **, too many non-detected constituent concentrations to estimate statistics with log-probablity regression]

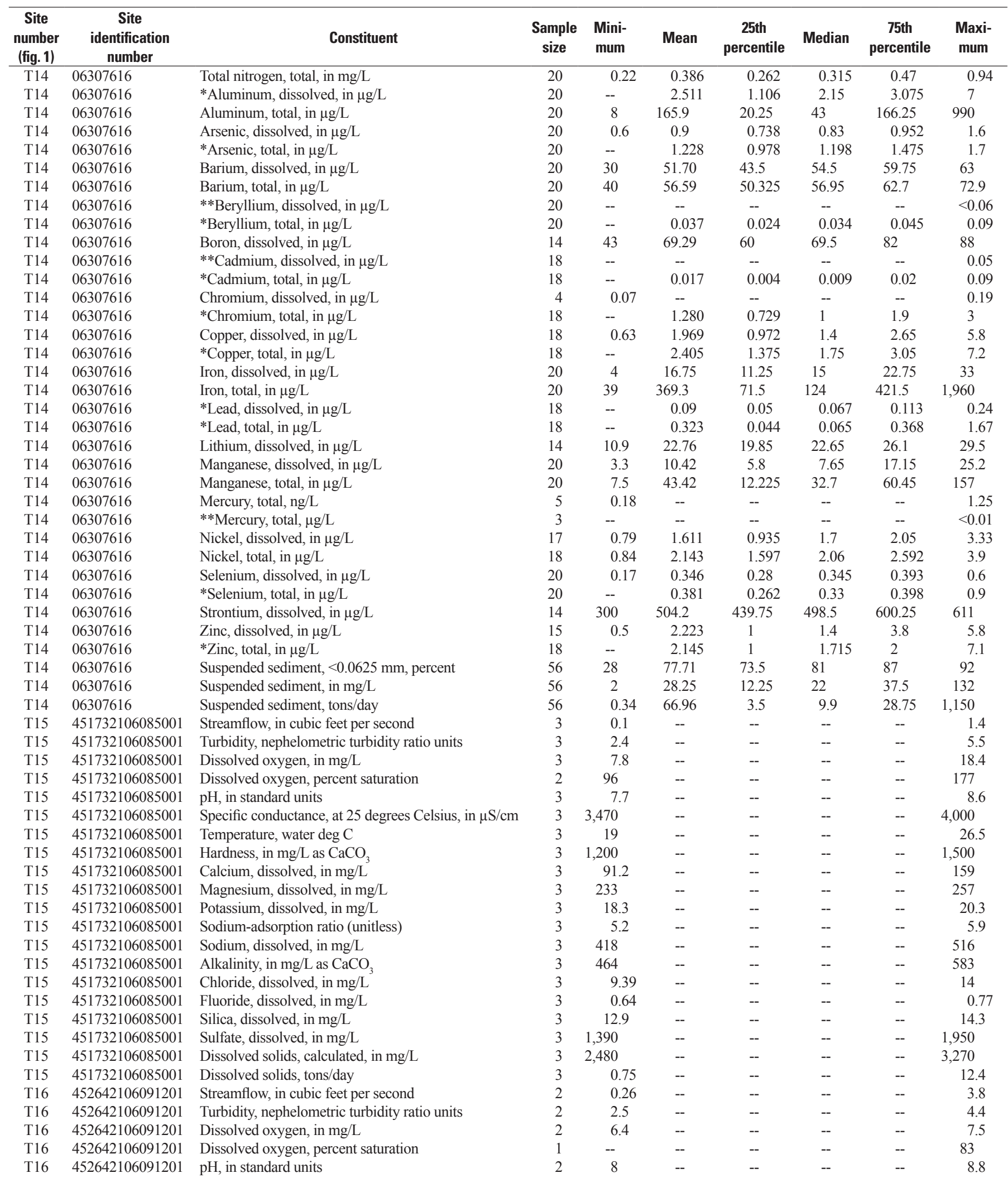


Appendix. Summary statistics for water-quality constituents, Powder River structural basin, Wyoming and Montana, water years 2005-08.-Continued

[--, value cannot be calculated; $\mathrm{mg} / \mathrm{L}$, milligrams per liter; $\mu \mathrm{S} / \mathrm{cm}$, microsiemens per centimeter at 25 degrees Celsius; deg $\mathrm{C}$, degrees Celsius; $\mathrm{CaCO}_{3}$, calcium carbonate; as $\mathrm{N}$, as nitrogen; as $\mathrm{P}$, as phosphorus; $\mu \mathrm{g} / \mathrm{L}$, micrograms per liter; $\mathrm{ng} / \mathrm{L}$, nanograms per liter; mm, millimeters; *, constituent statistics were estimated using a log-probability regression; **, too many non-detected constituent concentrations to estimate statistics with log-probablity regression]

\begin{tabular}{|c|c|c|c|c|c|c|c|c|c|}
\hline $\begin{array}{c}\text { Site } \\
\text { number } \\
\text { (fig. 1) }\end{array}$ & $\begin{array}{c}\text { Site } \\
\text { identification } \\
\text { number }\end{array}$ & Constituent & $\begin{array}{c}\text { Sample } \\
\text { size }\end{array}$ & $\begin{array}{l}\text { Mini- } \\
\text { mum }\end{array}$ & Mean & $\begin{array}{c}\text { 25th } \\
\text { percentile }\end{array}$ & Median & $\begin{array}{c}\text { 75th } \\
\text { percentile }\end{array}$ & $\begin{array}{c}\text { Maxi- } \\
\text { mum }\end{array}$ \\
\hline T16 & 452642106091201 & Specific conductance, at 25 degrees Celsius, in $\mu \mathrm{S} / \mathrm{cm}$ & 2 & 3,200 & -- & -- & -- & -- & 3,600 \\
\hline T16 & 452642106091201 & Temperature, water $\operatorname{deg} \mathrm{C}$ & 2 & 19.5 & -- & -- & -- & -- & 21.5 \\
\hline $\mathrm{T} 16$ & 452642106091201 & Calcium, dissolved, in $\mathrm{mg} / \mathrm{L}^{3}$ & 2 & 52 & -- & -- & -- & -- & 129 \\
\hline T16 & 452642106091201 & Magnesium, dissolved, in mg/L & 2 & 196 & -- & -- & -- & -- & 246 \\
\hline T16 & 452642106091201 & Potassium, dissolved, in $\mathrm{mg} / \mathrm{L}$ & 2 & 19.4 & -- & -- & -- & -- & 19.4 \\
\hline T16 & 452642106091201 & Alkalinity, in $\mathrm{mg} / \mathrm{L}$ as $\mathrm{CaCO}_{3}$ & 2 & 438 & -- & -- & -- & -- & 502 \\
\hline $\mathrm{T} 16$ & 452642106091201 & Chloride, dissolved, in $\mathrm{mg} / \mathrm{L}^{3}$ & 2 & 10.7 & -- & -- & -- & -- & 12.9 \\
\hline T16 & 452642106091201 & Fluoride, dissolved, in mg/L & 2 & 0.65 & -- & -- & -- & -- & 0.66 \\
\hline T16 & 452642106091201 & Silica, dissolved, in $\mathrm{mg} / \mathrm{L}$ & 2 & 1.16 & -- & -- & -- & -- & 7.43 \\
\hline T16 & 452642106091201 & Sulfate, dissolved, in $\mathrm{mg} / \mathrm{L}$ & 2 & 1,440 & -- & -- & -- & -- & 1,730 \\
\hline T16 & 452642106091201 & Dissolved solids, calculated, in $\mathrm{mg} / \mathrm{L}$ & 2 & 2,490 & -- & -- & -- & -- & 2,800 \\
\hline T17 & 06307740 & $\mathrm{pH}$, in standard units & 43 & 7.9 & 8.39 & 8.3 & 8.4 & 8.5 & 8.9 \\
\hline T17 & 06307740 & Specific conductance, at 25 degrees Celsius, in $\mu \mathrm{S} / \mathrm{cm}$ & 45 & 1,730 & 2,841 & 2,510 & 2,880 & 3,115 & 3,700 \\
\hline T17 & 06307740 & Temperature, water deg $\mathrm{C}$ & 45 & 0 & 14.16 & 7.5 & 14 & 22 & 29 \\
\hline T17 & 06307740 & Hardness, in $\mathrm{mg} / \mathrm{L}$ as $\mathrm{CaCO}_{3}$ & 43 & 480 & 822.1 & 720 & 820 & 910 & 1,100 \\
\hline T17 & 06307740 & Calcium, dissolved, in $\mathrm{mg} / \mathrm{L}$ & 43 & 50.1 & 79.69 & 67.3 & 79 & 87.5 & 122 \\
\hline T17 & 06307740 & Magnesium, dissolved, in $\mathrm{mg} / \mathrm{L}$ & 43 & 84 & 151.1 & 132 & 151 & 165 & 206 \\
\hline T17 & 06307740 & Potassium, dissolved, in $\mathrm{mg} / \mathrm{L}$ & 43 & 11.7 & 18.88 & 17.4 & 18.6 & 20.4 & 25.8 \\
\hline T17 & 06307740 & Sodium-adsorption ratio (unitless) & 43 & 4.6 & 6.005 & 5.5 & 6.1 & 6.4 & 6.9 \\
\hline $\mathrm{T} 17$ & 06307740 & Sodium, dissolved, in $\mathrm{mg} / \mathrm{L}$ & 43 & 233 & 396.4 & 334 & 406 & 443 & 520 \\
\hline T17 & 06307740 & Alkalinity, in $\mathrm{mg} / \mathrm{L}$ as $\mathrm{CaCO}_{3}$ & 43 & 350 & 549.8 & 508 & 554 & 592 & 807 \\
\hline $\mathrm{T} 17$ & 06307740 & Chloride, dissolved, in $\mathrm{mg} / \mathrm{L}^{3}$ & 43 & 7.12 & 11.79 & 10.1 & 11.7 & 13.1 & 16.1 \\
\hline T17 & 06307740 & Fluoride, dissolved, in mg/L & 43 & 0.58 & 0.9 & 0.83 & 0.91 & 0.97 & 1.2 \\
\hline T17 & 06307740 & Silica, dissolved, in $\mathrm{mg} / \mathrm{L}$ & 43 & 5.8 & 10.77 & 8.02 & 9.9 & 14 & 22.3 \\
\hline T17 & 06307740 & Total nitrogen, total, in $\mathrm{mg} / \mathrm{L}$ & 20 & 0.31 & 0.737 & 0.562 & 0.755 & 0.91 & 1.29 \\
\hline $\mathrm{T} 17$ & 06307740 & *Aluminum, dissolved, in $\mu \mathrm{g} / \mathrm{L}$ & 19 & -- & 1.984 & 1.5 & 1.8 & 2.112 & 4.9 \\
\hline T17 & 06307740 & Aluminum, total, in $\mu \mathrm{g} / \mathrm{L}$ & 19 & 37 & 349.5 & 104 & 299 & 522 & 1,020 \\
\hline T17 & 06307740 & Arsenic, dissolved, in $\mu \mathrm{g} / \mathrm{L}$ & 19 & 0.69 & 2.227 & 1.2 & 1.4 & 1.7 & 7.5 \\
\hline T17 & 06307740 & *Arsenic, total, in $\mu \mathrm{g} / \mathrm{L}$ & 19 & -- & 2.458 & 1.3 & 1.7 & 2.3 & 8.2 \\
\hline T17 & 06307740 & Barium, dissolved, in $\mu \mathrm{g} / \mathrm{L}$ & 19 & 11 & 33.21 & 25 & 31 & 39 & 61 \\
\hline T17 & 06307740 & Barium, total, in $\mu \mathrm{g} / \mathrm{L}$ & 19 & 11.2 & 41.14 & 29 & 38 & 50.4 & 76.8 \\
\hline T17 & 06307740 & **Beryllium, dissolved, in $\mu \mathrm{g} / \mathrm{L}$ & 19 & -- & -- & -- & -- & -- & $<0.12$ \\
\hline T17 & 06307740 & *Beryllium, total, in $\mu \mathrm{g} / \mathrm{L}$ & 19 & -- & 0.047 & 0.034 & 0.04 & 0.06 & 0.1 \\
\hline T17 & 06307740 & Boron, dissolved, in $\mu \mathrm{g} / \mathrm{L}$ & 14 & 357 & 495.6 & 407 & 498 & 541.5 & 744 \\
\hline T17 & 06307740 & *Cadmium, dissolved, in $\mu \mathrm{g} / \mathrm{L}$ & 17 & -- & 0.026 & 0.02 & 0.023 & 0.03 & 0.04 \\
\hline T17 & 06307740 & ${ }^{*}$ Cadmium, total, in $\mu \mathrm{g} / \mathrm{L}$ & 17 & -- & 0.034 & 0.02 & 0.03 & 0.05 & 0.06 \\
\hline $\mathrm{T} 17$ & 06307740 & **Chromium, dissolved, in $\mu \mathrm{g} / \mathrm{L}$ & 3 & -- & -- & -- & -- & -- & 0.45 \\
\hline T17 & 06307740 & *Chromium, total, in $\mu \mathrm{g} / \mathrm{L}$ & 17 & -- & 3.381 & 1.068 & 3 & 5.5 & 9 \\
\hline T17 & 06307740 & *Copper, dissolved, in $\mu \mathrm{g} / \mathrm{L}$ & 17 & -- & 3.893 & 1.3 & 3.3 & 4.9 & 11.3 \\
\hline T17 & 06307740 & ${ }^{*}$ Copper, total, in $\mu \mathrm{g} / \mathrm{L}$ & 17 & -- & 6.654 & 3.4 & 5.4 & 7.7 & 20.1 \\
\hline T17 & 06307740 & *Iron, dissolved, in $\mu \mathrm{g} / \mathrm{L}$ & 19 & -- & 20.40 & 12.82 & 16.646 & 28 & 43 \\
\hline $\mathrm{T} 17$ & 06307740 & Iron, total, in $\mu \mathrm{g} / \mathrm{L}$ & 19 & 220 & 849.4 & 361 & 802 & 1,100 & 2,220 \\
\hline $\mathrm{T} 17$ & 06307740 & *Lead, dissolved, in $\mu \mathrm{g} / \mathrm{L}$ & 17 & -- & 0.085 & 0.051 & 0.07 & 0.095 & 0.26 \\
\hline T17 & 06307740 & Lead, total, in $\mu \mathrm{g} / \mathrm{L}$ & 17 & 0.05 & 0.801 & 0.22 & 0.67 & 1.14 & 2.24 \\
\hline
\end{tabular}


Appendix. Summary statistics for water-quality constituents, Powder River structural basin, Wyoming and Montana, water years 2005-08.-Continued

[--, value cannot be calculated; $\mathrm{mg} / \mathrm{L}$, milligrams per liter; $\mu \mathrm{S} / \mathrm{cm}$, microsiemens per centimeter at 25 degrees Celsius; deg $\mathrm{C}$, degrees Celsius; $\mathrm{CaCO}_{3}$, calcium carbonate; as $\mathrm{N}$, as nitrogen; as $\mathrm{P}$, as phosphorus; $\mu \mathrm{g} / \mathrm{L}$, micrograms per liter; ng/L, nanograms per liter; mm, millimeters; *, constituent statistics were estimated using a log-probability regression; **, too many non-detected constituent concentrations to estimate statistics with log-probablity regression]

\begin{tabular}{|c|c|c|c|c|c|c|c|c|c|}
\hline $\begin{array}{c}\text { Site } \\
\text { number } \\
\text { (fig. 1) }\end{array}$ & $\begin{array}{c}\text { Site } \\
\text { identification } \\
\text { number }\end{array}$ & Constituent & $\begin{array}{l}\text { Sample } \\
\text { size }\end{array}$ & $\begin{array}{l}\text { Mini- } \\
\text { mum }\end{array}$ & Mean & $\begin{array}{c}\text { 25th } \\
\text { percentile }\end{array}$ & Median & $\begin{array}{c}\text { 75th } \\
\text { percentile }\end{array}$ & $\begin{array}{l}\text { Maxi- } \\
\text { mum }\end{array}$ \\
\hline T17 & 06307740 & Lithium, dissolved, in $\mu \mathrm{g} / \mathrm{L}$ & 14 & 80 & 111.1 & 94.1 & 107.5 & 128.75 & 162 \\
\hline T17 & 06307740 & Mercury, total, ng/L & 6 & 0.5 & 2.367 & 1.415 & 1.935 & 3.613 & 4.94 \\
\hline $\mathrm{T} 17$ & 06307740 & $* *$ Mercury, total, $\mu \mathrm{g} / \mathrm{L}$ & 3 & -- & -- & -- & -- & -- & $<0.01$ \\
\hline T17 & 06307740 & Nickel, dissolved, in $\mu \mathrm{g} / \mathrm{L}$ & 17 & 1.4 & 3.502 & 2.61 & 3.06 & 4.41 & 7.94 \\
\hline $\mathrm{T} 17$ & 06307740 & Selenium, total, in $\mu \mathrm{g} / \mathrm{L}$ & 19 & 0.45 & 1.126 & 0.82 & 0.94 & 1.5 & 2.1 \\
\hline T17 & 06307740 & Strontium, dissolved, in $\mu \mathrm{g} / \mathrm{L}$ & 14 & 1,120 & 1,675 & 1,325 & 1,695 & 1,978 & 2,430 \\
\hline $\mathrm{T} 17$ & 06307740 & $*$ Zinc, dissolved, in $\mu \mathrm{g} / \mathrm{L}$ & 17 & -- & 2.844 & 1.8 & 2.6 & 3.8 & 5.4 \\
\hline $\mathrm{T} 17$ & 06307740 & $*$ Zinc, total, in $\mu \mathrm{g} / \mathrm{L}$ & 17 & -- & 5.782 & 3 & 6 & 7 & 13 \\
\hline T17 & 06307740 & Suspended sediment, $<0.0625 \mathrm{~mm}$, percent & 38 & 45 & 81.63 & 73.5 & 82 & 95.25 & 98 \\
\hline T17 & 06307740 & Suspended sediment, in mg/L & 38 & 26 & 95.84 & 45 & 97.5 & 137 & 186 \\
\hline $\mathrm{T} 18$ & 06307830 & $\mathrm{pH}$, in standard units & 52 & 8 & 8.42 & 8.3 & 8.5 & 8.5 & 8.7 \\
\hline T18 & 06307830 & Specific conductance, at 25 degrees Celsius, in $\mu \mathrm{S} / \mathrm{cm}$ & 53 & 337 & 689.4 & 570.5 & 730 & 835 & 926 \\
\hline T18 & 06307830 & Temperature, water deg $\mathrm{C}$ & 53 & 0 & 14.59 & 7.75 & 15 & 23 & 29 \\
\hline T18 & 06307830 & Hardness, in $\mathrm{mg} / \mathrm{L}$ as $\mathrm{CaCO}_{3}$ & 52 & 150 & 273.3 & 222.5 & 280 & 327.5 & 380 \\
\hline T18 & 06307830 & Calcium, dissolved, in $\mathrm{mg} / \mathrm{L}^{3}$ & 52 & 32.5 & 51.16 & 43.5 & 50.7 & 58.675 & 69.8 \\
\hline T18 & 06307830 & Magnesium, dissolved, in $\mathrm{mg} / \mathrm{L}$ & 52 & 16.2 & 35.41 & 26.35 & 35.8 & 43.9 & 51.7 \\
\hline T18 & 06307830 & Potassium, dissolved, in mg/L & 52 & 2.24 & 4.067 & 3.453 & 3.945 & 4.807 & 7.31 \\
\hline T18 & 06307830 & Sodium-adsorption ratio (unitless) & 52 & 0.5 & 1.235 & 0.925 & 1.35 & 1.5 & 1.8 \\
\hline $\mathrm{T} 18$ & 06307830 & Sodium, dissolved, in $\mathrm{mg} / \mathrm{L}$ & 52 & 13.5 & 47.97 & 32.375 & 51.5 & 62.475 & 80.4 \\
\hline $\mathrm{T} 18$ & 06307830 & Alkalinity, in $\mathrm{mg} / \mathrm{L}$ as $\mathrm{CaCO}_{3}$ & 52 & 115 & 208.7 & 176.75 & 218.5 & 245.5 & 288 \\
\hline T18 & 06307830 & Chloride, dissolved, in $\mathrm{mg} / \mathrm{L}^{3}$ & 52 & 1.69 & 4.276 & 3.155 & 4.835 & 5.405 & 5.9 \\
\hline $\mathrm{T} 18$ & 06307830 & Fluoride, dissolved, in mg/L & 52 & 0.17 & 0.327 & 0.26 & 0.34 & 0.39 & 0.45 \\
\hline $\mathrm{T} 18$ & 06307830 & Silica, dissolved, in $\mathrm{mg} / \mathrm{L}$ & 52 & 1.41 & 4.732 & 3.572 & 4.555 & 5.775 & 7.58 \\
\hline $\mathrm{T} 18$ & 06307830 & Total nitrogen, total, in $\mathrm{mg} / \mathrm{L}$ & 18 & 0.17 & 0.423 & 0.245 & 0.305 & 0.425 & 1.36 \\
\hline $\mathrm{T} 18$ & 06307830 & *Aluminum, dissolved, in $\mu \mathrm{g} / \mathrm{L}$ & 17 & -- & 2.531 & 1.211 & 2 & 3.8 & 6.1 \\
\hline $\mathrm{T} 18$ & 06307830 & Aluminum, total, in $\mu \mathrm{g} / \mathrm{L}$ & 17 & 44 & 452.3 & 88.5 & 151 & 370.5 & 3,550 \\
\hline $\mathrm{T} 18$ & 06307830 & Arsenic, dissolved, in $\mu \mathrm{g} / \mathrm{L}$ & 17 & 0.6 & 0.828 & 0.695 & 0.8 & 0.885 & 1.3 \\
\hline $\mathrm{T} 18$ & 06307830 & *Arsenic, total, in $\mu \mathrm{g} / \mathrm{L}$ & 17 & -- & 1.349 & 0.99 & 1.1 & 1.45 & 3 \\
\hline T18 & 06307830 & Barium, dissolved, in $\mu \mathrm{g} / \mathrm{L}$ & 17 & 46 & 55.65 & 50 & 55 & 60.5 & 68 \\
\hline T18 & 06307830 & Barium, total, in $\mu \mathrm{g} / \mathrm{L}$ & 17 & 51.5 & 65.88 & 55.4 & 58.5 & 69.85 & 140 \\
\hline T18 & 06307830 & $* *$ Beryllium, dissolved, in $\mu \mathrm{g} / \mathrm{L}$ & 17 & -- & -- & -- & -- & -- & $<0.06$ \\
\hline T18 & 06307830 & *Beryllium, total, in $\mu \mathrm{g} / \mathrm{L}$ & 17 & -- & 0.081 & 0.037 & 0.05 & 0.093 & 0.4 \\
\hline T18 & 06307830 & Boron, dissolved, in $\mu \mathrm{g} / \mathrm{L}$ & 14 & 56 & 90.14 & 69 & 87 & 114 & 123 \\
\hline T18 & 06307830 & $* *$ Cadmium, dissolved, in $\mu \mathrm{g} / \mathrm{L}$ & 15 & -- & -- & -- & -- & -- & $<0.04$ \\
\hline T18 & 06307830 & $* *$ Cadmium, total, in $\mu \mathrm{g} / \mathrm{L}$ & 15 & -- & -- & -- & -- & -- & 0.17 \\
\hline T18 & 06307830 & Chromium, dissolved, in $\mu \mathrm{g} / \mathrm{L}$ & 1 & -- & -- & -- & -- & -- & 0.14 \\
\hline $\mathrm{T} 18$ & 06307830 & ${ }^{*}$ Chromium, total, in $\mu \mathrm{g} / \mathrm{L}$ & 15 & -- & 1.896 & 1 & 1.103 & 2.05 & 8 \\
\hline $\mathrm{T} 18$ & 06307830 & Copper, dissolved, in $\mu \mathrm{g} / \mathrm{L}$ & 15 & 0.61 & 1.938 & 1.3 & 1.8 & 2.6 & 3.4 \\
\hline T18 & 06307830 & Copper, total, in $\mu \mathrm{g} / \mathrm{L}$ & 15 & 1.1 & 3.320 & 1.5 & 2 & 4.6 & 13.1 \\
\hline $\mathrm{T} 18$ & 06307830 & *Iron, dissolved, in $\mu \mathrm{g} / \mathrm{L}$ & 17 & -- & 12.75 & 4.279 & 10 & 14.5 & 64 \\
\hline T18 & 06307830 & Iron, total, in $\mu \mathrm{g} / \mathrm{L}$ & 17 & 120 & 902.5 & 189.5 & 365 & 593.5 & 6,880 \\
\hline $\mathrm{T} 18$ & 06307830 & *Lead, dissolved, in $\mu \mathrm{g} / \mathrm{L}$ & 15 & -- & 0.096 & 0.06 & 0.075 & 0.16 & 0.2 \\
\hline T18 & 06307830 & Lead, total, in $\mu \mathrm{g} / \mathrm{L}$ & 15 & 0.1 & 0.879 & 0.15 & 0.27 & 0.48 & 6.98 \\
\hline
\end{tabular}


Appendix. Summary statistics for water-quality constituents, Powder River structural basin, Wyoming and Montana, water years 2005-08. - Continued

[--, value cannot be calculated; $\mathrm{mg} / \mathrm{L}$, milligrams per liter; $\mu \mathrm{S} / \mathrm{cm}$, microsiemens per centimeter at 25 degrees Celsius; deg $\mathrm{C}$, degrees Celsius; $\mathrm{CaCO}_{3}$, calcium carbonate; as $\mathrm{N}$, as nitrogen; as $\mathrm{P}$, as phosphorus; $\mu \mathrm{g} / \mathrm{L}$, micrograms per liter; $\mathrm{ng} / \mathrm{L}$, nanograms per liter; mm, millimeters; *, constituent statistics were estimated using a log-probability regression; **, too many non-detected constituent concentrations to estimate statistics with log-probablity regression]

\begin{tabular}{|c|c|c|c|c|c|c|c|c|c|}
\hline $\begin{array}{c}\text { Site } \\
\text { number } \\
\text { (fig. 1) }\end{array}$ & $\begin{array}{c}\text { Site } \\
\text { identification } \\
\text { number }\end{array}$ & Constituent & $\begin{array}{l}\text { Sample } \\
\text { size }\end{array}$ & $\begin{array}{l}\text { Mini- } \\
\text { mum }\end{array}$ & Mean & $\begin{array}{c}\text { 25th } \\
\text { percentile }\end{array}$ & Median & $\begin{array}{c}\text { 75th } \\
\text { percentile }\end{array}$ & $\begin{array}{l}\text { Maxi- } \\
\text { mum }\end{array}$ \\
\hline T18 & 06307830 & Lithium, dissolved, in $\mu \mathrm{g} / \mathrm{L}$ & 14 & 14.5 & 23.89 & 19.675 & 23.25 & 26.3 & 42.6 \\
\hline $\mathrm{T} 18$ & 06307830 & Manganese, dissolved, in $\mu \mathrm{g} / \mathrm{L}$ & 17 & 1 & 8.041 & 3.4 & 7 & 10.8 & 24.9 \\
\hline T18 & 06307830 & Mercury, total, ng/L & 5 & 0.83 & -- & -- & -- & -- & 2.02 \\
\hline T18 & 06307830 & $* *$ Mercury, total, $\mu \mathrm{g} / \mathrm{L}$ & 3 & -- & -- & -- & -- & -- & $<0.01$ \\
\hline T18 & 06307830 & Nickel, dissolved, in $\mu \mathrm{g} / \mathrm{L}$ & 15 & 0.89 & 2.016 & 1.4 & 2.03 & 2.7 & 3.47 \\
\hline $\mathrm{T} 18$ & 06307830 & *Selenium, total, in $\mu \mathrm{g} / \mathrm{L}$ & 17 & -- & 0.437 & 0.275 & 0.37 & 0.435 & 1.4 \\
\hline $\mathrm{T} 18$ & 06307830 & Strontium, dissolved, in $\mu \mathrm{g} / \mathrm{L}$ & 14 & 346 & 580.7 & 499.75 & 587 & 684.25 & 787 \\
\hline $\mathrm{T} 18$ & 06307830 & Zinc, dissolved, in $\mu \mathrm{g} / \mathrm{L}$ & 15 & 0.39 & 2.393 & 0.9 & 1.6 & 3 & 9.4 \\
\hline T18 & 06307830 & Zinc, total, in $\mu \mathrm{g} / \mathrm{L}$ & 15 & 1 & 4.407 & 2 & 2 & 3 & 27 \\
\hline T18 & 06307830 & Suspended sediment, $<0.0625 \mathrm{~mm}$, percent & 45 & 46 & 82.84 & 77.5 & 86 & 90 & 96 \\
\hline T18 & 06307830 & Suspended sediment, in $\mathrm{mg} / \mathrm{L}$ & 45 & 3 & 65.69 & 23 & 37 & 56.5 & 513 \\
\hline T19 & 06308400 & Specific conductance, at 25 degrees Celsius, in $\mu \mathrm{S} / \mathrm{cm}$ & 37 & 263 & 1,270 & 509.5 & 1,010 & 1,650 & 4,430 \\
\hline T19 & 06308400 & Temperature, water deg C & 37 & 0 & 12.96 & 5.25 & 13 & 19.75 & 30 \\
\hline T19 & 06308400 & Hardness, in $\mathrm{mg} / \mathrm{L}$ as $\mathrm{CaCO}_{3}$ & 37 & 4 & 181.5 & 36 & 110 & 205 & 970 \\
\hline T19 & 06308400 & Calcium, dissolved, in $\mathrm{mg} / \mathrm{L}^{3}$ & 37 & 0.97 & 31.97 & 8.695 & 24.8 & 38.35 & 132 \\
\hline T19 & 06308400 & Magnesium, dissolved, in $\mathrm{mg} / \mathrm{L}$ & 37 & 0.366 & 24.64 & 2.99 & 11.9 & 26.2 & 167 \\
\hline T19 & 06308400 & Potassium, dissolved, in $\mathrm{mg} / \mathrm{L}$ & 37 & 1.48 & 7.578 & 4.42 & 7.23 & 8.91 & 22.4 \\
\hline T19 & 06308400 & Sodium-adsorption ratio (unitless) & 37 & 2.6 & 8.332 & 6.05 & 7.7 & 10 & 15 \\
\hline T19 & 06308400 & Sodium, dissolved, in mg/L & 37 & 35.9 & 221.3 & 96.05 & 180 & 304.5 & 745 \\
\hline T19 & 06308400 & Alkalinity, in $\mathrm{mg} / \mathrm{L}$ as $\mathrm{CaCO}_{3}$ & 37 & 80 & 210.4 & 115 & 185 & 287 & 435 \\
\hline T19 & 06308400 & Chloride, dissolved, in $\mathrm{mg} / \mathrm{L}$ & 37 & 0.91 & 4.370 & 1.74 & 3.18 & 5.76 & 14.5 \\
\hline T19 & 06308400 & Fluoride, dissolved, in mg/L & 37 & 0.17 & 0.405 & 0.33 & 0.4 & 0.47 & 0.66 \\
\hline T19 & 06308400 & Silica, dissolved, in $\mathrm{mg} / \mathrm{L}$ & 36 & 0.74 & 7.469 & 6.543 & 7.665 & 8.672 & 12.9 \\
\hline T19 & 06308400 & Sulfate, dissolved, in $\mathrm{mg} / \mathrm{L}$ & 37 & 40.2 & 450.1 & 134 & 294 & 588 & 2,090 \\
\hline T19 & 06308400 & Aluminum, dissolved, in $\mu \mathrm{g} / \mathrm{L}$ & 14 & 1.6 & 9.364 & 1.85 & 10.3 & 14.825 & 18.9 \\
\hline T19 & 06308400 & Aluminum, total, in $\mu \mathrm{g} / \mathrm{L}$ & 14 & 124 & 17,083 & 352.25 & 5,865 & 34,275 & 63,900 \\
\hline T19 & 06308400 & Arsenic, dissolved, in $\mu \mathrm{g} / \mathrm{L}$ & 14 & 0.78 & 1.200 & 0.97 & 1.2 & 1.425 & 1.6 \\
\hline T19 & 06308400 & *Arsenic, total, in $\mu \mathrm{g} / \mathrm{L}$ & 14 & -- & 5.618 & 1.316 & 2.7 & 7.95 & 29 \\
\hline T19 & 06308400 & Barium, dissolved, in $\mu \mathrm{g} / \mathrm{L}$ & 14 & 13 & 66.71 & 21.5 & 56.5 & 77.25 & 247 \\
\hline T19 & 06308400 & Barium, total, in $\mu \mathrm{g} / \mathrm{L}$ & 14 & 65 & 382.1 & 93.175 & 153.5 & 667.25 & 1,310 \\
\hline T19 & 06308400 & **Beryllium, dissolved, in $\mu \mathrm{g} / \mathrm{L}$ & 14 & -- & -- & -- & -- & -- & $<0.12$ \\
\hline T19 & 06308400 & *Beryllium, total, in $\mu \mathrm{g} / \mathrm{L}$ & 14 & -- & 2.313 & 0.064 & 0.435 & 4.755 & 8.73 \\
\hline T19 & 06308400 & Boron, dissolved, in $\mu \mathrm{g} / \mathrm{L}$ & 10 & 46 & 144.0 & 53.75 & 121 & 259.5 & 274 \\
\hline T19 & 06308400 & *Cadmium, dissolved, in $\mu \mathrm{g} / \mathrm{L}$ & 13 & -- & 0.034 & 0.023 & 0.03 & 0.041 & 0.06 \\
\hline T19 & 06308400 & ${ }^{*}$ Cadmium, total, in $\mu \mathrm{g} / \mathrm{L}$ & 13 & -- & 0.666 & 0.029 & 0.2 & 1.565 & 2.2 \\
\hline T19 & 06308400 & Chromium, dissolved, in $\mu \mathrm{g} / \mathrm{L}$ & 3 & 0.14 & -- & -- & -- & -- & 0.5 \\
\hline T19 & 06308400 & ${ }^{*}$ Chromium, total, in $\mu \mathrm{g} / \mathrm{L}$ & 13 & -- & 27.83 & 1.4 & 11 & 47 & 123 \\
\hline T19 & 06308400 & Copper, dissolved, in $\mu \mathrm{g} / \mathrm{L}$ & 13 & 2.3 & 7.815 & 5.25 & 6 & 11.05 & 17 \\
\hline T19 & 06308400 & Copper, total, in $\mu \mathrm{g} / \mathrm{L}$ & 13 & 4.4 & 51.25 & 8.4 & 19.5 & 102.1 & 159 \\
\hline T19 & 06308400 & *Iron, dissolved, in $\mu \mathrm{g} / \mathrm{L}$ & 14 & -- & 36.85 & 9.889 & 23 & 52.5 & 147 \\
\hline T19 & 06308400 & Iron, total, in $\mu \mathrm{g} / \mathrm{L}$ & 14 & 94 & 21,353 & 385.75 & 5,340 & 42,275 & 97,700 \\
\hline T19 & 06308400 & *Lead, dissolved, in $\mu \mathrm{g} / \mathrm{L}$ & 13 & -- & 0.383 & 0.128 & 0.18 & 0.43 & 1.68 \\
\hline T19 & 06308400 & Lead, total, in $\mu \mathrm{g} / \mathrm{L}$ & 13 & 0.23 & 41.52 & 0.795 & 13.5 & 77.35 & 181 \\
\hline T19 & 06308400 & Lithium, dissolved, in $\mu \mathrm{g} / \mathrm{L}$ & 10 & 3.1 & 15.28 & 4.575 & 9 & 28.325 & 38.5 \\
\hline
\end{tabular}


Appendix. Summary statistics for water-quality constituents, Powder River structural basin, Wyoming and Montana, water years 2005-08.-Continued

[--, value cannot be calculated; $\mathrm{mg} / \mathrm{L}$, milligrams per liter; $\mu \mathrm{S} / \mathrm{cm}$, microsiemens per centimeter at 25 degrees Celsius; deg C, degrees Celsius; $\mathrm{CaCO}_{3}$, calcium carbonate; as $\mathrm{N}$, as nitrogen; as $\mathrm{P}$, as phosphorus; $\mu \mathrm{g} / \mathrm{L}$, micrograms per liter; $\mathrm{ng} / \mathrm{L}$, nanograms per liter; mm, millimeters; *, constituent statistics were estimated using a log-probability regression; **, too many non-detected constituent concentrations to estimate statistics with log-probablity regression]

\begin{tabular}{|c|c|c|c|c|c|c|c|c|c|}
\hline $\begin{array}{c}\text { Site } \\
\text { number } \\
\text { (fig. 1) }\end{array}$ & $\begin{array}{c}\text { Site } \\
\text { identification } \\
\text { number }\end{array}$ & Constituent & $\begin{array}{c}\text { Sample } \\
\text { size }\end{array}$ & $\begin{array}{l}\text { Mini- } \\
\text { mum }\end{array}$ & Mean & $\begin{array}{c}\text { 25th } \\
\text { percentile }\end{array}$ & Median & $\begin{array}{c}\text { 75th } \\
\text { percentile }\end{array}$ & $\begin{array}{l}\text { Maxi- } \\
\text { mum }\end{array}$ \\
\hline T19 & 06308400 & Manganese, dissolved, in $\mu \mathrm{g} / \mathrm{L}$ & 14 & 0.7 & 5.279 & 1.825 & 4.2 & 6.425 & 15.9 \\
\hline T19 & 06308400 & Manganese, total, in $\mu \mathrm{g} / \mathrm{L}$ & 14 & 25.8 & 401.3 & 44.2 & 91.05 & 760.25 & 1,620 \\
\hline $\mathrm{T} 19$ & 06308400 & $* *$ Mercury, total, $\mu \mathrm{g} / \mathrm{L}$ & 2 & -- & -- & -- & -- & -- & 0.474 \\
\hline $\mathrm{T} 19$ & 06308400 & Nickel, dissolved, in $\mu \mathrm{g} / \mathrm{L}$ & 13 & 3.1 & 5.742 & 4.3 & 6.08 & 7.18 & 7.88 \\
\hline T19 & 06308400 & Nickel, total, in $\mu \mathrm{g} / \mathrm{L}$ & 13 & 3.2 & 51.78 & 6.875 & 18 & 102.3 & 186 \\
\hline T19 & 06308400 & Strontium, dissolved, in $\mu \mathrm{g} / \mathrm{L}$ & 10 & 23 & 494.5 & 131 & 216 & 993.5 & 1,610 \\
\hline T19 & 06308400 & $*$ Zinc, dissolved, in $\mu \mathrm{g} / \mathrm{L}$ & 13 & -- & 7.891 & 2.05 & 2.582 & 13.2 & 34.5 \\
\hline T19 & 06308400 & Zinc, total, in $\mu \mathrm{g} / \mathrm{L}$ & 13 & 3.9 & 152.1 & 5.05 & 48 & 312.5 & 603 \\
\hline T19 & 06308400 & Suspended sediment, $<0.0625 \mathrm{~mm}$, percent & 33 & 89 & 97.79 & 98.5 & 99 & 99 & 99 \\
\hline T19 & 06308400 & Suspended sediment, in $\mathrm{mg} / \mathrm{L}$ & 33 & 12 & 3,600 & 58 & 666 & 5,505 & 20,100 \\
\hline T19 & 06308400 & Suspended sediment, tons/day & 33 & 0 & 694.1 & 0.055 & 2.7 & 131.5 & 12,400 \\
\hline P1 & 434056106244101 & Specific conductance, at 25 degrees Celsius, in $\mu \mathrm{S} / \mathrm{cm}$ & 3 & 2,090 & -- & -- & -- & -- & 3,470 \\
\hline P1 & 434056106244101 & Temperature, water deg $\mathrm{C}$ & 3 & 27 & -- & -- & -- & -- & 32.8 \\
\hline P1 & 434056106244101 & Hardness, in $\mathrm{mg} / \mathrm{L}$ as $\mathrm{CaCO}_{3}$ & 3 & 710 & -- & -- & -- & -- & 1,100 \\
\hline P1 & 434056106244101 & Calcium, dissolved, in $\mathrm{mg} / \mathrm{L}$ & 3 & 170 & -- & -- & -- & -- & 287 \\
\hline P1 & 434056106244101 & Magnesium, dissolved, in $\mathrm{mg} / \mathrm{L}$ & 3 & 70.8 & -- & -- & -- & -- & 89.4 \\
\hline P1 & 434056106244101 & Potassium, dissolved, in $\mathrm{mg} / \mathrm{L}$ & 3 & 7.33 & -- & -- & -- & -- & 14.2 \\
\hline P1 & 434056106244101 & Sodium-adsorption ratio (unitless) & 3 & 3.3 & -- & -- & -- & -- & 5.7 \\
\hline P1 & 434056106244101 & Sodium, dissolved, in $\mathrm{mg} / \mathrm{L}$ & 3 & 209 & -- & -- & -- & -- & 430 \\
\hline P1 & 434056106244101 & Alkalinity, in $\mathrm{mg} / \mathrm{L}$ as $\mathrm{CaCO}_{3}$ & 3 & 177 & -- & -- & -- & -- & 225 \\
\hline P1 & 434056106244101 & Chloride, dissolved, in $\mathrm{mg} / \mathrm{L}$ & 3 & 92.5 & -- & -- & -- & -- & 139 \\
\hline P1 & 434056106244101 & Fluoride, dissolved, in $\mathrm{mg} / \mathrm{L}$ & 3 & 0.46 & -- & -- & -- & -- & 0.83 \\
\hline P1 & 434056106244101 & Silica, dissolved, in $\mathrm{mg} / \mathrm{L}$ & 3 & 4.93 & -- & -- & -- & -- & 11.9 \\
\hline P1 & 434056106244101 & Sulfate, dissolved, in $\mathrm{mg} / \mathrm{L}$ & 3 & 722 & -- & -- & -- & -- & 1,610 \\
\hline $\mathrm{P} 2$ & 434124106192401 & Temperature, water deg C & 3 & 22.1 & -- & -- & -- & -- & 32.7 \\
\hline $\mathrm{P} 2$ & 434124106192401 & Hardness, in $\mathrm{mg} / \mathrm{L}$ as $\mathrm{CaCO}_{3}$ & 3 & 590 & -- & -- & -- & -- & 730 \\
\hline P2 & 434124106192401 & Calcium, dissolved, in $\mathrm{mg} / \mathrm{L}$ & 3 & 113 & -- & -- & -- & -- & 177 \\
\hline $\mathrm{P} 2$ & 434124106192401 & Magnesium, dissolved, in $\mathrm{mg} / \mathrm{L}$ & 3 & 69.7 & -- & -- & -- & -- & 79 \\
\hline P2 & 434124106192401 & Potassium, dissolved, in $\mathrm{mg} / \mathrm{L}$ & 3 & 15.6 & -- & -- & -- & -- & 28.7 \\
\hline P2 & 434124106192401 & Sodium-adsorption ratio (unitless) & 3 & 8.9 & -- & -- & -- & -- & 17 \\
\hline P2 & 434124106192401 & Sodium, dissolved, in mg/L & 3 & 554 & -- & -- & -- & -- & 932 \\
\hline P2 & 434124106192401 & Alkalinity, in $\mathrm{mg} / \mathrm{L}$ as $\mathrm{CaCO}_{3}$ & 3 & 175 & -- & -- & -- & -- & 288 \\
\hline P2 & 434124106192401 & Chloride, dissolved, in $\mathrm{mg} / \mathrm{L}$ & 3 & 444 & -- & -- & -- & -- & 943 \\
\hline P2 & 434124106192401 & Fluoride, dissolved, in mg/L & 3 & 1.03 & -- & -- & -- & -- & 1.99 \\
\hline P2 & 434124106192401 & Silica, dissolved, in $\mathrm{mg} / \mathrm{L}$ & 3 & 4.46 & -- & -- & -- & -- & 12.5 \\
\hline P2 & 434124106192401 & Sulfate, dissolved, in $\mathrm{mg} / \mathrm{L}$ & 3 & 1,030 & -- & -- & -- & -- & 1,310 \\
\hline P2 & 434124106192401 & Dissolved solids, calculated, in mg/L & 3 & 2,450 & -- & -- & -- & -- & 3,490 \\
\hline P2 & 434124106192401 & Dissolved solids, tons/day & 3 & 48.3 & -- & -- & -- & -- & 253 \\
\hline P3 & 435453106104701 & Streamflow, in cubic feet per second & 4 & 0.22 & -- & -- & -- & -- & 84 \\
\hline P3 & 435453106104701 & Turbidity, nephelometric turbidity ratio units & 4 & 3.1 & -- & -- & -- & -- & 690 \\
\hline P3 & 435453106104701 & Dissolved oxygen, in mg/L & 4 & 6.2 & -- & -- & -- & -- & 11.4 \\
\hline P3 & 435453106104701 & Dissolved oxygen, percent saturation & 3 & 106 & -- & -- & -- & -- & 172 \\
\hline P3 & 435453106104701 & $\mathrm{pH}$, in standard units & 4 & 7.8 & -- & -- & -- & -- & 8.4 \\
\hline P3 & 435453106104701 & Specific conductance, at 25 degrees Celsius, in $\mu \mathrm{S} / \mathrm{cm}$ & 4 & 2,980 & -- & -- & -- & -- & 4,940 \\
\hline
\end{tabular}


Appendix. Summary statistics for water-quality constituents, Powder River structural basin, Wyoming and Montana, water years 2005-08.-Continued

[--, value cannot be calculated; $\mathrm{mg} / \mathrm{L}$, milligrams per liter; $\mu \mathrm{S} / \mathrm{cm}$, microsiemens per centimeter at 25 degrees Celsius; deg $\mathrm{C}$, degrees $\mathrm{Celsius}^{\mathrm{C}} \mathrm{CaCO}_{3}$, calcium carbonate; as $\mathrm{N}$, as nitrogen; as $\mathrm{P}$, as phosphorus; $\mu \mathrm{g} / \mathrm{L}$, micrograms per liter; $\mathrm{ng} / \mathrm{L}$, nanograms per liter; mm, millimeters; *, constituent statistics were estimated using a log-probability regression; **, too many non-detected constituent concentrations to estimate statistics with log-probablity regression]

\begin{tabular}{|c|c|c|c|c|c|c|c|c|c|}
\hline $\begin{array}{c}\text { Site } \\
\text { number } \\
\text { (fig. 1) }\end{array}$ & $\begin{array}{c}\text { Site } \\
\text { identification } \\
\text { number } \\
\end{array}$ & Constituent & $\begin{array}{l}\text { Sample } \\
\text { size }\end{array}$ & $\begin{array}{l}\text { Mini- } \\
\text { mum }\end{array}$ & Mean & $\begin{array}{c}\text { 25th } \\
\text { percentile }\end{array}$ & Median & $\begin{array}{c}\text { 75th } \\
\text { percentile }\end{array}$ & $\begin{array}{c}\text { Maxi- } \\
\text { mum }\end{array}$ \\
\hline P3 & 435453106104701 & Temperature, water deg C & 4 & 20.1 & -- & -- & -- & -- & 29.1 \\
\hline P3 & 435453106104701 & Hardness, in $\mathrm{mg} / \mathrm{L}$ as $\mathrm{CaCO}_{3}$ & 4 & 620 & -- & -- & -- & -- & 960 \\
\hline P3 & 435453106104701 & Magnesium, dissolved, in $\mathrm{mg} / \mathrm{L}$ & 4 & 64.3 & -- & -- & -- & -- & 111 \\
\hline P3 & 435453106104701 & Potassium, dissolved, in $\mathrm{mg} / \mathrm{L}$ & 4 & 11.3 & -- & -- & -- & -- & 21.2 \\
\hline P3 & 435453106104701 & Sodium-adsorption ratio (unitless) & 4 & 7.1 & -- & -- & -- & -- & 13 \\
\hline P3 & 435453106104701 & Chloride, dissolved, in $\mathrm{mg} / \mathrm{L}^{3}$ & 4 & 344 & -- & -- & -- & -- & 733 \\
\hline P3 & 435453106104701 & Fluoride, dissolved, in mg/L & 4 & 0.65 & -- & -- & -- & -- & 1.19 \\
\hline P3 & 435453106104701 & Silica, dissolved, in $\mathrm{mg} / \mathrm{L}$ & 4 & 3.02 & -- & -- & -- & -- & 12.4 \\
\hline P3 & 435453106104701 & Sulfate, dissolved, in $\mathrm{mg} / \mathrm{L}$ & 4 & 880 & -- & -- & -- & -- & 1,550 \\
\hline P3 & 435453106104701 & Dissolved solids, calculated, in mg/L & 4 & 1,980 & -- & -- & -- & -- & 3,350 \\
\hline P3 & 435453106104701 & Dissolved solids, tons/day & 4 & 1.89 & -- & -- & -- & -- & 476 \\
\hline P4 & 440919106091401 & Specific conductance, at 25 degrees Celsius, in $\mu \mathrm{S} / \mathrm{cm}$ & 4 & 2,860 & -- & -- & -- & -- & 4,610 \\
\hline P4 & 440919106091401 & Temperature, water deg C & 4 & 18.6 & -- & -- & -- & -- & 30.3 \\
\hline P4 & 440919106091401 & Hardness, in $\mathrm{mg} / \mathrm{L}$ as $\mathrm{CaCO}_{3}$ & 4 & 150 & -- & -- & -- & -- & 700 \\
\hline P4 & 440919106091401 & Calcium, dissolved, in $\mathrm{mg} / \mathrm{L}^{3}$ & 4 & 13.9 & -- & -- & -- & -- & 153 \\
\hline P4 & 440919106091401 & Magnesium, dissolved, in $\mathrm{mg} / \mathrm{L}$ & 4 & 29.1 & -- & -- & -- & -- & 100 \\
\hline P4 & 440919106091401 & Potassium, dissolved, in $\mathrm{mg} / \mathrm{L}$ & 4 & 11.9 & -- & -- & -- & -- & 25.7 \\
\hline P4 & 440919106091401 & Sodium-adsorption ratio (unitless) & 4 & 7.3 & -- & -- & -- & -- & 25 \\
\hline P4 & 440919106091401 & Sodium, dissolved, in mg/L & 4 & 407 & -- & -- & -- & -- & 866 \\
\hline P4 & 440919106091401 & Alkalinity, in $\mathrm{mg} / \mathrm{L}$ as $\mathrm{CaCO}_{3}$ & 4 & 224 & -- & -- & -- & -- & 1,540 \\
\hline P4 & 440919106091401 & Chloride, dissolved, in $\mathrm{mg} / \mathrm{L}$ & 4 & 69.6 & -- & -- & -- & -- & 537 \\
\hline P4 & 440919106091401 & Fluoride, dissolved, in mg/L & 4 & 0.61 & -- & -- & -- & -- & 1.62 \\
\hline P4 & 440919106091401 & Silica, dissolved, in $\mathrm{mg} / \mathrm{L}$ & 4 & 5.01 & -- & -- & -- & -- & 8.74 \\
\hline P4 & 440919106091401 & Sulfate, dissolved, in $\mathrm{mg} / \mathrm{L}$ & 4 & 210 & -- & -- & -- & -- & 1,260 \\
\hline P5 & 442538106082001 & Temperature, water deg C & 4 & 18.7 & -- & -- & -- & -- & 32.9 \\
\hline P5 & 442538106082001 & Hardness, in $\mathrm{mg} / \mathrm{L}$ as $\mathrm{CaCO}_{3}$ & 4 & 400 & -- & -- & -- & -- & 620 \\
\hline P5 & 442538106082001 & Calcium, dissolved, in $\mathrm{mg} / \mathrm{L}^{3}$ & 4 & 78.8 & -- & -- & -- & -- & 135 \\
\hline P5 & 442538106082001 & Magnesium, dissolved, in $\mathrm{mg} / \mathrm{L}$ & 4 & 50 & -- & -- & -- & -- & 72.6 \\
\hline P5 & 442538106082001 & Potassium, dissolved, in $\mathrm{mg} / \mathrm{L}$ & 4 & 12.2 & -- & -- & -- & -- & 14.8 \\
\hline P5 & 442538106082001 & Sodium-adsorption ratio (unitless) & 4 & 7.1 & -- & -- & -- & -- & 11 \\
\hline P5 & 442538106082001 & Sodium, dissolved, in $\mathrm{mg} / \mathrm{L}$ & 4 & 392 & -- & -- & -- & -- & 524 \\
\hline P5 & 442538106082001 & Alkalinity, in $\mathrm{mg} / \mathrm{L}$ as $\mathrm{CaCO}_{3}$ & 4 & 202 & -- & -- & -- & -- & 342 \\
\hline P5 & 442538106082001 & Chloride, dissolved, in $\mathrm{mg} / \mathrm{L}^{3}$ & 4 & 212 & -- & -- & -- & -- & 365 \\
\hline P5 & 442538106082001 & Fluoride, dissolved, in $\mathrm{mg} / \mathrm{L}$ & 4 & 0.67 & -- & -- & -- & -- & 0.92 \\
\hline P5 & 442538106082001 & Silica, dissolved, in $\mathrm{mg} / \mathrm{L}$ & 4 & 4.32 & -- & -- & -- & -- & 8.15 \\
\hline P5 & 442538106082001 & Sulfate, dissolved, in $\mathrm{mg} / \mathrm{L}$ & 4 & 871 & -- & -- & -- & -- & 1,070 \\
\hline P5 & 442538106082001 & Dissolved solids, calculated, in $\mathrm{mg} / \mathrm{L}$ & 4 & 1,870 & -- & -- & -- & -- & 2,320 \\
\hline P5 & 442538106082001 & Dissolved solids, tons/day & 4 & 9.19 & -- & -- & -- & -- & 500 \\
\hline P6 & 441532106251301 & Streamflow, in cubic feet per second & 3 & 0 & -- & -- & -- & -- & 37 \\
\hline P6 & 441532106251301 & Turbidity, nephelometric turbidity ratio units & 3 & 11 & -- & -- & -- & -- & 240 \\
\hline P6 & 441532106251301 & Dissolved oxygen, in $\mathrm{mg} / \mathrm{L}$ & 3 & 6.8 & -- & -- & -- & -- & 8.8 \\
\hline P6 & 441532106251301 & Dissolved oxygen, percent saturation & 2 & 96 & -- & -- & -- & -- & 114 \\
\hline P6 & 441532106251301 & $\mathrm{pH}$, in standard units & 3 & 7.9 & -- & -- & -- & -- & 8.2 \\
\hline P6 & 441532106251301 & Specific conductance, at 25 degrees Celsius, in $\mu \mathrm{S} / \mathrm{cm}$ & 3 & 827 & -- & -- & -- & -- & 3,500 \\
\hline
\end{tabular}


Appendix. Summary statistics for water-quality constituents, Powder River structural basin, Wyoming and Montana, water years 2005-08.-Continued

[--, value cannot be calculated; $\mathrm{mg} / \mathrm{L}$, milligrams per liter; $\mu \mathrm{S} / \mathrm{cm}$, microsiemens per centimeter at 25 degrees Celsius; deg $\mathrm{C}$, degrees $\mathrm{Celsius}^{\mathrm{C}} \mathrm{CaCO}_{3}$, calcium carbonate; as $\mathrm{N}$, as nitrogen; as $\mathrm{P}$, as phosphorus; $\mu \mathrm{g} / \mathrm{L}$, micrograms per liter; $\mathrm{ng} / \mathrm{L}$, nanograms per liter; mm, millimeters; *, constituent statistics were estimated using a log-probability regression; **, too many non-detected constituent concentrations to estimate statistics with log-probablity regression]

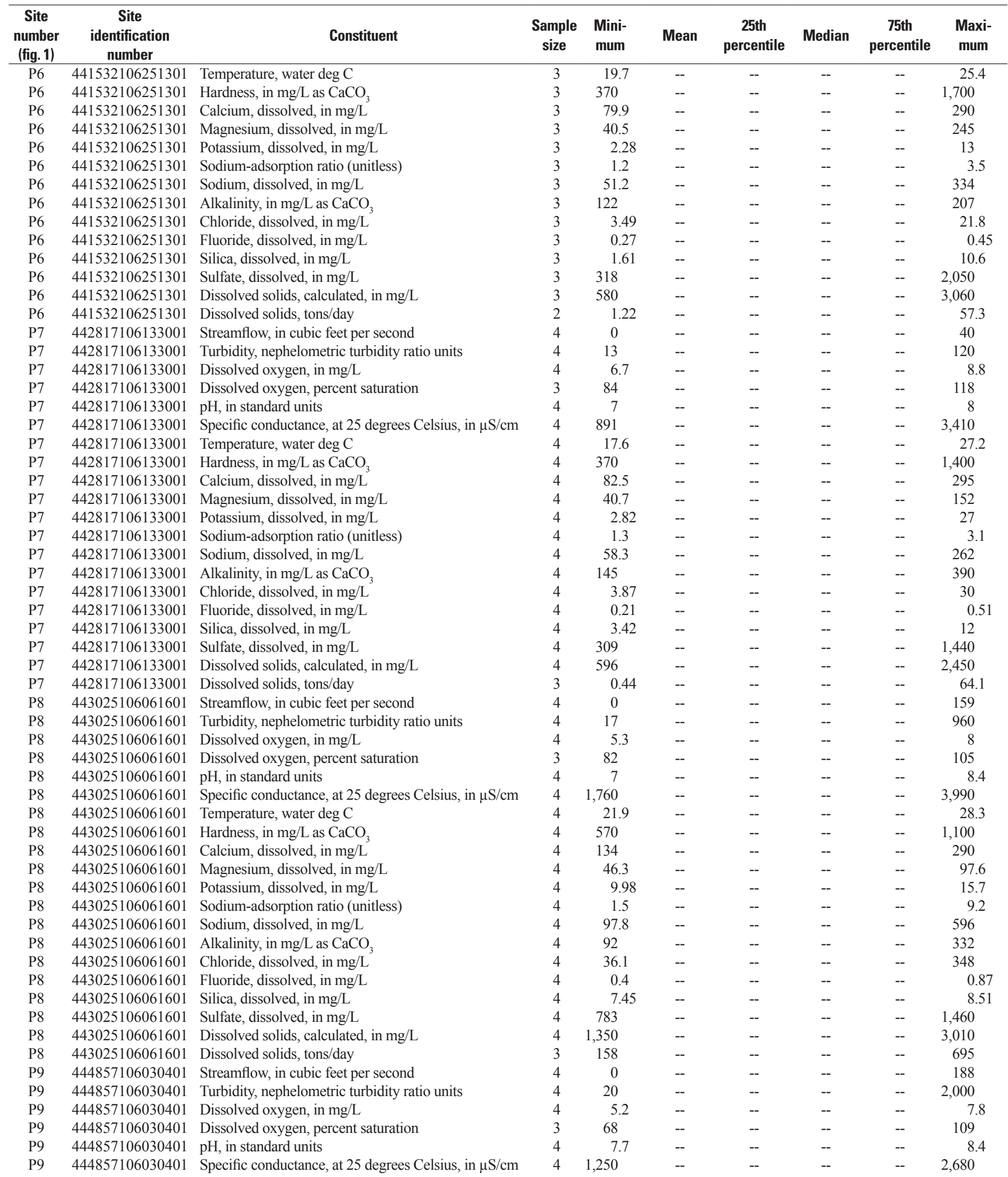


Appendix. Summary statistics for water-quality constituents, Powder River structural basin, Wyoming and Montana, water years 2005-08.-Continued

[--, value cannot be calculated; $\mathrm{mg} / \mathrm{L}$, milligrams per liter; $\mu \mathrm{S} / \mathrm{cm}$, microsiemens per centimeter at 25 degrees Celsius; deg $\mathrm{C}$, degrees Celsius; $\mathrm{CaCO}_{3}$, calcium carbonate; as $\mathrm{N}$, as nitrogen; as $\mathrm{P}$, as phosphorus; $\mu \mathrm{g} / \mathrm{L}$, micrograms per liter; $\mathrm{ng} / \mathrm{L}$, nanograms per liter; mm, millimeters; *, constituent statistics were estimated using a log-probability regression; **, too many non-detected constituent concentrations to estimate statistics with log-probablity regression]

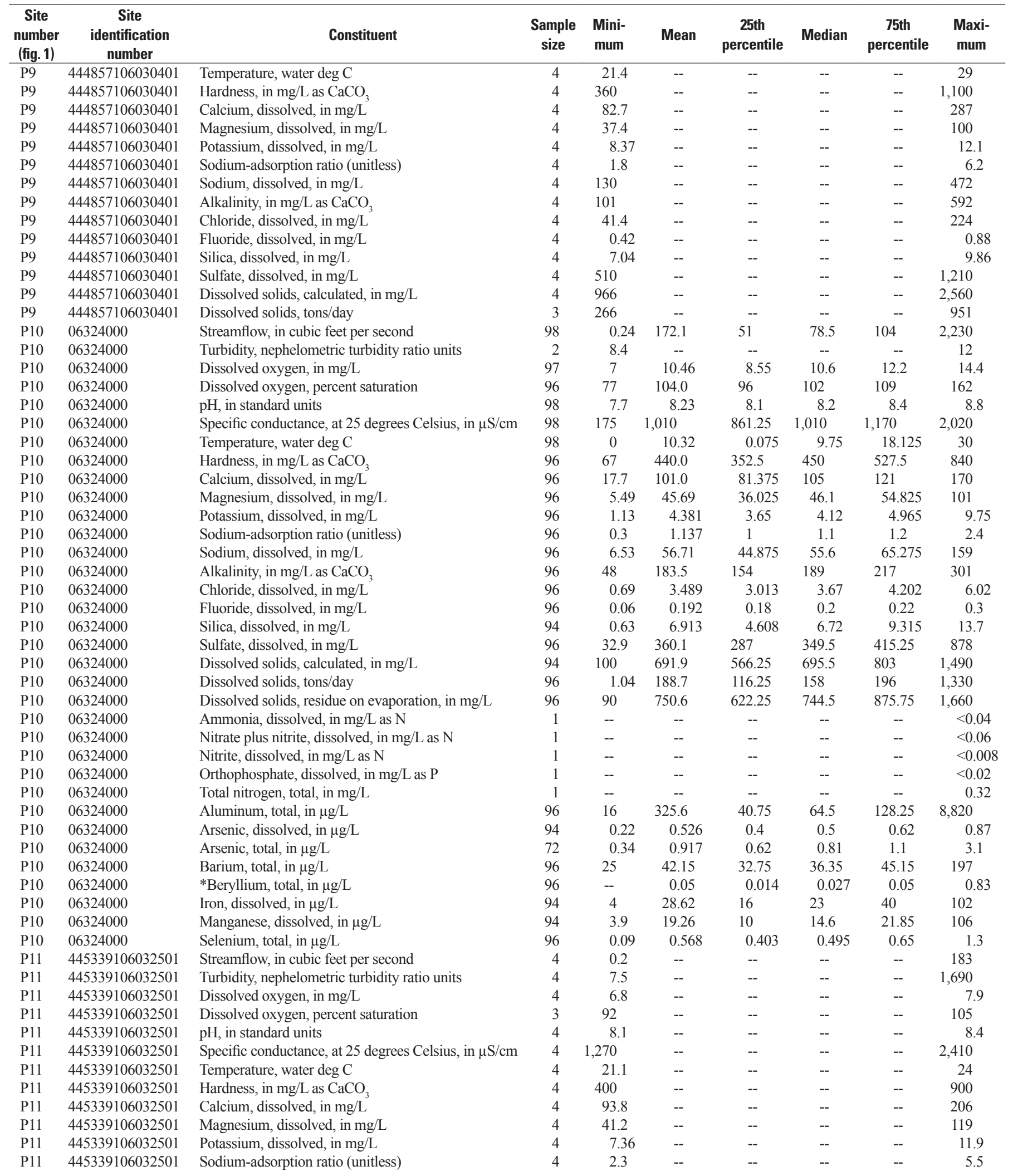


Appendix. Summary statistics for water-quality constituents, Powder River structural basin, Wyoming and Montana, water years 2005-08.-Continued

[--, value cannot be calculated; $\mathrm{mg} / \mathrm{L}$, milligrams per liter; $\mu \mathrm{S} / \mathrm{cm}$, microsiemens per centimeter at 25 degrees Celsius; deg C, degrees Celsius; $\mathrm{CaCO}_{3}$, calcium carbonate; as $\mathrm{N}$, as nitrogen; as $\mathrm{P}$, as phosphorus; $\mu \mathrm{g} / \mathrm{L}$, micrograms per liter; $\mathrm{ng} / \mathrm{L}$, nanograms per liter; mm, millimeters; *, constituent statistics were estimated using a log-probability regression; **, too many non-detected constituent concentrations to estimate statistics with log-probablity regression]

\begin{tabular}{|c|c|c|c|c|c|c|c|c|c|}
\hline $\begin{array}{l}\text { Site } \\
\text { number } \\
\text { (fig. 1) }\end{array}$ & $\begin{array}{c}\text { Site } \\
\text { identification } \\
\text { number }\end{array}$ & Constituent & $\begin{array}{l}\text { Sample } \\
\text { size }\end{array}$ & $\begin{array}{l}\text { Mini- } \\
\text { mum }\end{array}$ & Mean & $\begin{array}{c}\text { 25th } \\
\text { percentile }\end{array}$ & Median & $\begin{array}{c}\text { 75th } \\
\text { percentile }\end{array}$ & $\begin{array}{l}\text { Maxi- } \\
\text { mum }\end{array}$ \\
\hline P11 & 445339106032501 & Sodium, dissolved, in $\mathrm{mg} / \mathrm{L}$ & 4 & 128 & -- & -- & -- & -- & 302 \\
\hline P11 & 445339106032501 & Alkalinity, in $\mathrm{mg} / \mathrm{L}$ as $\mathrm{CaCO}_{3}$ & 4 & 132 & -- & -- & -- & -- & 205 \\
\hline P11 & 445339106032501 & Fluoride, dissolved, in $\mathrm{mg} / \mathrm{L}$ & 4 & 0.39 & -- & -- & -- & -- & 0.55 \\
\hline P11 & 445339106032501 & Silica, dissolved, in $\mathrm{mg} / \mathrm{L}$ & 4 & 1.1 & -- & -- & -- & -- & 6.64 \\
\hline P11 & 445339106032501 & Sulfate, dissolved, in $\mathrm{mg} / \mathrm{L}$ & 4 & 481 & -- & -- & -- & -- & 1,090 \\
\hline P12 & 06324500 & Streamflow, in cubic feet per second & 98 & 0 & 375.0 & 90.75 & 192 & 297 & 5,220 \\
\hline $\mathrm{P} 12$ & 06324500 & Turbidity, nephelometric turbidity ratio units & 2 & 41 & -- & -- & -- & -- & 110 \\
\hline P12 & 06324500 & Dissolved oxygen, in $\mathrm{mg} / \mathrm{L}$ & 98 & 6.2 & 10.24 & 8.675 & 9.9 & 11.9 & 15.7 \\
\hline P12 & 06324500 & Dissolved oxygen, percent saturation & 98 & 75 & 102.1 & 97 & 101.5 & 106.25 & 148 \\
\hline P12 & 06324500 & $\mathrm{pH}$, in standard units & 97 & 7.6 & 8.29 & 8.2 & 8.3 & 8.4 & 8.7 \\
\hline P12 & 06324500 & Specific conductance, at 25 degrees Celsius, in $\mu \mathrm{S} / \mathrm{cm}$ & 98 & 500 & 1,855 & 1,525 & 1,855 & 2,083 & 4,300 \\
\hline $\mathrm{P} 12$ & 06324500 & Sodium-adsorption ratio (unitless) & 96 & 1.5 & 4.183 & 3.45 & 4.2 & 4.6 & 9 \\
\hline P12 & 06324500 & Sodium, dissolved, in $\mathrm{mg} / \mathrm{L}$ & 96 & 46.7 & 218.6 & 162.5 & 217.5 & 257 & 520 \\
\hline P12 & 06324500 & Acid neutralizing capacity, in $\mathrm{mg} / \mathrm{L}$ as $\mathrm{CaCO}_{3}$ & 91 & 119 & 276.7 & 201 & 265 & 327 & 697 \\
\hline P12 & 06324500 & Alkalinity, in $\mathrm{mg} / \mathrm{L}$ as $\mathrm{CaCO}_{3}$ & 96 & 81 & 227.6 & 176.5 & 216.5 & 259.75 & 441 \\
\hline P12 & 06324500 & Chloride, dissolved, in $\mathrm{mg} / \mathrm{L}^{3}$ & 96 & 10 & 103.7 & 70.325 & 104 & 127.5 & 232 \\
\hline P12 & 06324500 & Fluoride, dissolved, in mg/L & 96 & 0.23 & 0.524 & 0.44 & 0.54 & 0.62 & 0.99 \\
\hline P12 & 06324500 & Silica, dissolved, in $\mathrm{mg} / \mathrm{L}$ & 96 & 0.4 & 7.314 & 5.795 & 7.06 & 9.04 & 14.1 \\
\hline P12 & 06324500 & Sulfate, dissolved, in $\mathrm{mg} / \mathrm{L}$ & 96 & 129 & 595.5 & 476.5 & 567.5 & 665.5 & 1,640 \\
\hline P12 & 06324500 & Dissolved solids, calculated, in mg/L & 96 & 302 & 1,237 & 979.75 & 1,255 & 1,428 & 2,650 \\
\hline $\mathrm{P} 12$ & 06324500 & Dissolved solids, tons/day & 96 & 4.29 & 916.9 & 383.25 & 640.5 & 1,045 & 8,880 \\
\hline $\mathrm{P} 12$ & 06324500 & Dissolved solids, residue on evaporation, in $\mathrm{mg} / \mathrm{L}$ & 96 & 314 & 1,310 & 1,060 & 1,315 & 1,495 & 2,820 \\
\hline $\mathrm{P} 12$ & 06324500 & *Ammonia, dissolved, in $\mathrm{mg} / \mathrm{L}$ as $\mathrm{N}$ & 95 & -- & 0.041 & 0.008 & 0.015 & 0.065 & 0.221 \\
\hline P12 & 06324500 & *Nitrate plus nitrite, dissolved, in $\mathrm{mg} / \mathrm{L}$ as $\mathrm{N}$ & 95 & -- & 0.301 & 0.058 & 0.222 & 0.462 & 2.33 \\
\hline $\mathrm{P} 12$ & 06324500 & *Beryllium, total, in $\mu \mathrm{g} / \mathrm{L}$ & 96 & -- & 0.69 & 0.07 & 0.315 & 0.68 & 5.92 \\
\hline $\mathrm{P} 12$ & 06324500 & Boron, total, in $\mu \mathrm{g} / \mathrm{L}$ & 48 & 58 & 199.6 & 164 & 197.5 & 223.75 & 392 \\
\hline $\mathrm{P} 12$ & 06324500 & Cadmium, total, in $\mu \mathrm{g} / \mathrm{L}$ & 48 & 0.01 & 0.521 & 0.062 & 0.18 & 0.445 & 5 \\
\hline P12 & 06324500 & Chromium, total, in $\mu \mathrm{g} / \mathrm{L}$ & 48 & 0.26 & 12.00 & 1.425 & 5.3 & 11.25 & 74.2 \\
\hline P12 & 06324500 & Copper, total, in $\mu \mathrm{g} / \mathrm{L}$ & 48 & 1.2 & 20.56 & 5.45 & 10.5 & 25.15 & 102 \\
\hline P12 & 06324500 & *Iron, dissolved, in $\mu \mathrm{g} / \mathrm{L}$ & 96 & -- & 6.425 & 4 & 5.155 & 7.258 & 44 \\
\hline P12 & 06324500 & Iron, total, in $\mu \mathrm{g} / \mathrm{L}$ & 48 & 86 & 17,606 & 1,470 & 6,910 & 14,700 & 121,000 \\
\hline P12 & 06324500 & Lead, total, in $\mu \mathrm{g} / \mathrm{L}$ & 48 & 0.12 & 16.29 & 1.35 & 5.83 & 14 & 134 \\
\hline P12 & 06324500 & Manganese, dissolved, in $\mu \mathrm{g} / \mathrm{L}$ & 96 & 0.3 & 4.175 & 1.625 & 3.45 & 5.55 & 30.2 \\
\hline P12 & 06324500 & Manganese, total, in $\mu \mathrm{g} / \mathrm{L}$ & 48 & 14.4 & 371.1 & 38.125 & 124.5 & 356.75 & 3,330 \\
\hline P12 & 06324500 & Mercury, total, $\mu \mathrm{g} / \mathrm{L}$ & 4 & 0.016 & -- & -- & -- & -- & 0.182 \\
\hline P12 & 06324500 & Nickel, total, in $\mu \mathrm{g} / \mathrm{L}$ & 48 & 1.8 & 23.15 & 5.083 & 9.375 & 19.35 & 149 \\
\hline P12 & 06324500 & Selenium, total, in $\mu \mathrm{g} / \mathrm{L}$ & 96 & 0.33 & 1.937 & 1.2 & 1.5 & 2.275 & 11.3 \\
\hline P12 & 06324500 & Zinc, total, in $\mu \mathrm{g} / \mathrm{L}$ & 48 & 2 & 67.70 & 6.875 & 26.25 & 67.75 & 418 \\
\hline P12 & 06324500 & Suspended sediment, $<0.0625 \mathrm{~mm}$, percent & 91 & 45 & 89.44 & 85 & 96 & 98 & 99 \\
\hline P12 & 06324500 & Suspended sediment, in mg/L & 91 & 11 & 1,044 & 179 & 417 & 866 & 11,000 \\
\hline $\mathrm{P} 12$ & 06324500 & Suspended sediment, tons/day & 91 & 0.02 & 2,884 & 44 & 208 & 740 & 102,000 \\
\hline P13 & 06324710 & Streamflow, in cubic feet per second & 4 & 1.1 & -- & -- & -- & -- & 222 \\
\hline P13 & 06324710 & Turbidity, nephelometric turbidity ratio units & 4 & 3 & -- & -- & -- & -- & 100 \\
\hline P13 & 06324710 & Dissolved oxygen, in mg/L & 4 & 7.4 & -- & -- & -- & -- & 9.5 \\
\hline
\end{tabular}


Appendix. Summary statistics for water-quality constituents, Powder River structural basin, Wyoming and Montana, water years 2005-08.-Continued

[--, value cannot be calculated; $\mathrm{mg} / \mathrm{L}$, milligrams per liter; $\mu \mathrm{S} / \mathrm{cm}$, microsiemens per centimeter at 25 degrees Celsius; deg $\mathrm{C}$, degrees Celsius; $\mathrm{CaCO}_{3}$, calcium carbonate; as $\mathrm{N}$, as nitrogen; as $\mathrm{P}$, as phosphorus; $\mu \mathrm{g} / \mathrm{L}$, micrograms per liter; $\mathrm{ng} / \mathrm{L}$, nanograms per liter; mm, millimeters; *, constituent statistics were estimated using a log-probability regression; **, too many non-detected constituent concentrations to estimate statistics with log-probablity regression]

\begin{tabular}{|c|c|c|c|c|c|c|c|c|c|}
\hline $\begin{array}{c}\begin{array}{c}\text { Site } \\
\text { number } \\
\text { (fig. 1) }\end{array} \\
\end{array}$ & $\begin{array}{c}\text { Site } \\
\text { identification } \\
\text { number }\end{array}$ & Constituent & $\begin{array}{c}\text { Sample } \\
\text { size }\end{array}$ & $\begin{array}{l}\text { Mini- } \\
\text { mum }\end{array}$ & Mean & $\begin{array}{c}\text { 25th } \\
\text { percentile }\end{array}$ & Median & $\begin{array}{c}\text { 75th } \\
\text { percentile }\end{array}$ & $\begin{array}{c}\text { Maxi- } \\
\text { mum }\end{array}$ \\
\hline P13 & 06324710 & Dissolved oxygen, percent saturation & 3 & 108 & -- & -- & -- & -- & 136 \\
\hline P13 & 06324710 & Temperature, water deg C & 4 & 22.5 & -- & -- & -- & -- & 28 \\
\hline P13 & 06324710 & Hardness, in $\mathrm{mg} / \mathrm{L}$ as $\mathrm{CaCO}_{3}$ & 4 & 570 & -- & -- & -- & -- & 1,400 \\
\hline P13 & 06324710 & Calcium, dissolved, in $\mathrm{mg} / \mathrm{L}$ & 4 & 130 & -- & -- & -- & -- & 304 \\
\hline P13 & 06324710 & Sodium-adsorption ratio (unitless) & 4 & 3.4 & -- & -- & -- & -- & 7.4 \\
\hline P13 & 06324710 & Sodium, dissolved, in mg/L & 4 & 188 & -- & -- & -- & -- & 639 \\
\hline P13 & 06324710 & Alkalinity, in $\mathrm{mg} / \mathrm{L}$ as $\mathrm{CaCO}_{3}$ & 4 & 198 & -- & -- & -- & -- & 250 \\
\hline P13 & 06324710 & Chloride, dissolved, in $\mathrm{mg} / \mathrm{L}$ & 4 & 72.9 & -- & -- & -- & -- & 235 \\
\hline P13 & 06324710 & Fluoride, dissolved, in mg/L & 4 & 0.31 & -- & -- & -- & -- & 0.5 \\
\hline P13 & 06324710 & Silica, dissolved, in $\mathrm{mg} / \mathrm{L}$ & 4 & 8.46 & -- & -- & -- & -- & 11.5 \\
\hline P14 & 06324790 & Dissolved oxygen, in mg/L & 2 & 10 & -- & -- & -- & -- & 11.2 \\
\hline P14 & 06324790 & Dissolved oxygen, percent saturation & 1 & -- & -- & -- & -- & -- & 147 \\
\hline P14 & 06324790 & $\mathrm{pH}$, in standard units & 2 & 8.1 & -- & -- & -- & -- & 8.5 \\
\hline P14 & 06324790 & Specific conductance, at 25 degrees Celsius, in $\mu \mathrm{S} / \mathrm{cm}$ & 2 & 2,050 & -- & -- & -- & -- & 2,060 \\
\hline P14 & 06324790 & Temperature, water deg $\mathrm{C}$ & 2 & 20.8 & -- & -- & -- & -- & 22.8 \\
\hline P14 & 06324790 & Hardness, in $\mathrm{mg} / \mathrm{L}$ as $\mathrm{CaCO}_{3}$ & 3 & 730 & -- & -- & -- & -- & 1,100 \\
\hline P14 & 06324790 & Calcium, dissolved, in $\mathrm{mg} / \mathrm{L}$ & 3 & 144 & -- & -- & -- & -- & 239 \\
\hline P14 & 06324790 & Magnesium, dissolved, in $\mathrm{mg} / \mathrm{L}$ & 3 & 89.7 & -- & -- & -- & -- & 115 \\
\hline P14 & 06324790 & Potassium, dissolved, in mg/L & 3 & 22.6 & -- & -- & -- & -- & 38.8 \\
\hline P14 & 06324790 & Sodium-adsorption ratio (unitless) & 3 & 1.5 & -- & -- & -- & -- & 3.2 \\
\hline P14 & 06324790 & Sodium, dissolved, in mg/L & 3 & 106 & -- & -- & -- & -- & 201 \\
\hline P14 & 06324790 & Alkalinity, in $\mathrm{mg} / \mathrm{L}$ as $\mathrm{CaCO}_{3}$ & 3 & 146 & -- & -- & -- & -- & 259 \\
\hline P14 & 06324790 & Chloride, dissolved, in $\mathrm{mg} / \mathrm{L}^{3}$ & 3 & 41.3 & -- & -- & -- & -- & 88.6 \\
\hline P15 & 06324970 & Dissolved oxygen, percent saturation & 48 & 55 & 102.8 & 95.25 & 101.5 & 112 & 136 \\
\hline P15 & 06324970 & $\mathrm{pH}$, in standard units & 50 & 7.6 & 8.12 & 8 & 8.1 & 8.3 & 8.5 \\
\hline P15 & 06324970 & Specific conductance, at 25 degrees Celsius, in $\mu \mathrm{S} / \mathrm{cm}$ & 50 & 500 & 3,116 & 2,285 & 3,310 & 3,945 & 5,170 \\
\hline P15 & 06324970 & Temperature, water deg C & 50 & 0 & 10.99 & 0.5 & 12.2 & 19.5 & 24.8 \\
\hline P15 & 06324970 & Hardness, in $\mathrm{mg} / \mathrm{L}$ as $\mathrm{CaCO}_{3}$ & 48 & 81 & 800.4 & 565 & 865 & 990 & 1,400 \\
\hline P15 & 06324970 & Calcium, dissolved, in $\mathrm{mg} / \mathrm{L}$ & 48 & 18.7 & 154.5 & 111.5 & 161 & 187.5 & 298 \\
\hline P15 & 06324970 & Magnesium, dissolved, in mg/L & 48 & 8.24 & 100.6 & 69.275 & 111.5 & 125.75 & 186 \\
\hline P15 & 06324970 & Potassium, dissolved, in $\mathrm{mg} / \mathrm{L}$ & 48 & 6.53 & 18.21 & 15.35 & 18.5 & 21.3 & 28.3 \\
\hline P15 & 06324970 & Sodium-adsorption ratio (unitless) & 48 & 1.9 & 6.748 & 5.325 & 7 & 8 & 10 \\
\hline P15 & 06324970 & Sodium, dissolved, in $\mathrm{mg} / \mathrm{L}$ & 48 & 54.5 & 445.1 & 326 & 479.5 & 589.25 & 836 \\
\hline P15 & 06324970 & Acid neutralizing capacity, in $\mathrm{mg} / \mathrm{L}$ as $\mathrm{CaCO}_{3}$ & 47 & 98 & 357.2 & 276 & 370 & 420 & 728 \\
\hline P15 & 06324970 & Alkalinity, in $\mathrm{mg} / \mathrm{L}$ as $\mathrm{CaCO}_{3}$ & 48 & 82 & 347.2 & 263 & 361.5 & 410.25 & 734 \\
\hline P15 & 06324970 & Chloride, dissolved, in $\mathrm{mg} / \mathrm{L}^{3}$ & 48 & 6.75 & 69.70 & 38.1 & 52.6 & 79.425 & 298 \\
\hline P15 & 06324970 & Fluoride, dissolved, in mg/L & 48 & 0.21 & 0.662 & 0.555 & 0.675 & 0.748 & 1.09 \\
\hline P15 & 06324970 & Silica, dissolved, in $\mathrm{mg} / \mathrm{L}$ & 48 & 1.92 & 9.288 & 7.483 & 9.12 & 11.15 & 15.2 \\
\hline P15 & 06324970 & Sulfate, dissolved, in $\mathrm{mg} / \mathrm{L}$ & 48 & 138 & 1,334 & 901.75 & 1,395 & 1,770 & 2,420 \\
\hline P15 & 06324970 & Dissolved solids, calculated, in $\mathrm{mg} / \mathrm{L}$ & 48 & 299 & 2,340 & 1,625 & 2,525 & 3,033 & 4,200 \\
\hline P15 & 06324970 & Dissolved solids, tons/day & 48 & 0.2 & 54.88 & 8.868 & 24.9 & 62.975 & 422 \\
\hline P15 & 06324970 & Dissolved solids, residue on evaporation, in $\mathrm{mg} / \mathrm{L}$ & 48 & 324 & 2,485 & 1,725 & 2,635 & 3,188 & 4,400 \\
\hline P15 & 06324970 & *Ammonia, dissolved, in $\mathrm{mg} / \mathrm{L}$ as $\mathrm{N}$ & 38 & -- & 0.018 & 0.009 & 0.012 & 0.02 & 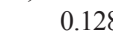 \\
\hline
\end{tabular}


Appendix. Summary statistics for water-quality constituents, Powder River structural basin, Wyoming and Montana, water years 2005-08.-Continued

[--, value cannot be calculated; $\mathrm{mg} / \mathrm{L}$, milligrams per liter; $\mu \mathrm{S} / \mathrm{cm}$, microsiemens per centimeter at 25 degrees Celsius; deg C, degrees Celsius; $\mathrm{CaCO}_{3}$, calcium carbonate; as $\mathrm{N}$, as nitrogen; as $\mathrm{P}$, as phosphorus; $\mu \mathrm{g} / \mathrm{L}$, micrograms per liter; $\mathrm{ng} / \mathrm{L}$, nanograms per liter; mm, millimeters; *, constituent statistics were estimated using a log-probability regression; **, too many non-detected constituent concentrations to estimate statistics with log-probablity regression]

\begin{tabular}{|c|c|c|c|c|c|c|c|c|c|}
\hline $\begin{array}{l}\text { Site } \\
\text { number } \\
\text { (fig. 1) }\end{array}$ & $\begin{array}{c}\text { Site } \\
\text { identification } \\
\text { number }\end{array}$ & Constituent & $\begin{array}{l}\text { Sample } \\
\text { size }\end{array}$ & $\begin{array}{l}\text { Mini- } \\
\text { mum }\end{array}$ & Mean & $\begin{array}{c}\text { 25th } \\
\text { percentile }\end{array}$ & Median & $\begin{array}{c}\text { 75th } \\
\text { percentile }\end{array}$ & $\begin{array}{l}\text { Maxi- } \\
\text { mum }\end{array}$ \\
\hline P15 & 06324970 & *Nitrate plus nitrite, dissolved, in $\mathrm{mg} / \mathrm{L}$ as $\mathrm{N}$ & 38 & -- & 0.052 & 0.014 & 0.027 & 0.05 & 0.49 \\
\hline P15 & 06324970 & *Nitrite, dissolved, in $\mathrm{mg} / \mathrm{L}$ as $\mathrm{N}$ & 38 & -- & 0.002 & 0.001 & 0.001 & 0.002 & 0.012 \\
\hline P15 & 06324970 & Total nitrogen, total, in $\mathrm{mg} / \mathrm{L}$ & 38 & 0.33 & 0.836 & 0.46 & 0.565 & 0.928 & 3.95 \\
\hline P15 & 06324970 & Aluminum, total, in $\mu \mathrm{g} / \mathrm{L}$ & 48 & 91 & 1,564 & 242.5 & 430 & 1,435 & 33,900 \\
\hline P15 & 06324970 & Arsenic, dissolved, in $\mu \mathrm{g} / \mathrm{L}$ & 48 & 0.43 & 0.93 & 0.69 & 0.84 & 1.1 & 2.3 \\
\hline P15 & 06324970 & *Beryllium, total, in $\mu \mathrm{g} / \mathrm{L}$ & 48 & -- & 0.236 & 0.058 & 0.081 & 0.138 & 6.27 \\
\hline P15 & 06324970 & Boron, total, in $\mu \mathrm{g} / \mathrm{L}$ & 48 & 55 & 214.6 & 148.5 & 194.5 & 261.25 & 615 \\
\hline P15 & 06324970 & *Cadmium, total, in $\mu \mathrm{g} / \mathrm{L}$ & 47 & -- & 0.088 & 0.021 & 0.04 & 0.05 & 2.2 \\
\hline P15 & 06324970 & ${ }^{*}$ Chromium, total, in $\mu \mathrm{g} / \mathrm{L}$ & 47 & -- & 2.027 & 0.6 & 0.86 & 1.7 & 39 \\
\hline P15 & 06324970 & ${ }^{*}$ Copper, total, in $\mu \mathrm{g} / \mathrm{L}$ & 47 & -- & 10.18 & 2.35 & 6.4 & 12.8 & 96.9 \\
\hline P15 & 06324970 & *Iron, dissolved, in $\mu \mathrm{g} / \mathrm{L}$ & 48 & -- & 18.21 & 9.139 & 14 & 18.024 & 136 \\
\hline P15 & 06324970 & Selenium, total, in $\mu \mathrm{g} / \mathrm{L}$ & 48 & 0.28 & 0.958 & 0.535 & 0.64 & 1.35 & 2.5 \\
\hline P15 & 06324970 & $*$ Zinc, total, in $\mu \mathrm{g} / \mathrm{L}$ & 47 & -- & 17.27 & 3.9 & 7 & 12 & 410 \\
\hline P15 & 06324970 & Suspended sediment, $<0.0625 \mathrm{~mm}$, percent & 25 & 55 & 89.76 & 83 & 96 & 98 & 100 \\
\hline P15 & 06324970 & Suspended sediment, in mg/L & 45 & 35 & 308.2 & 72 & 128 & 157 & 7,750 \\
\hline P15 & 06324970 & Suspended sediment, tons/day & 45 & 0 & 13.43 & 0.47 & 1.2 & 4.1 & 322 \\
\hline P16 & 06325000 & Streamflow, in cubic feet per second & 2 & 7.6 & -- & -- & -- & -- & 8.1 \\
\hline P16 & 06325000 & Turbidity, nephelometric turbidity ratio units & 2 & 23 & -- & -- & -- & -- & 810 \\
\hline P16 & 06325000 & Dissolved oxygen, in mg/L & 2 & 6.4 & -- & -- & -- & -- & 6.8 \\
\hline P16 & 06325000 & Dissolved oxygen, percent saturation & 2 & 76 & -- & -- & -- & -- & 87 \\
\hline P16 & 06325000 & $\mathrm{pH}$, in standard units & 2 & 7.9 & -- & -- & -- & -- & 8.1 \\
\hline P16 & 06325000 & Specific conductance, at 25 degrees Celsius, in $\mu \mathrm{S} / \mathrm{cm}$ & 2 & 2,720 & -- & -- & -- & -- & 4,090 \\
\hline P16 & 06325000 & Temperature, water deg C & 2 & 17.5 & -- & -- & -- & -- & 21 \\
\hline P16 & 06325000 & Hardness, in $\mathrm{mg} / \mathrm{L}$ as $\mathrm{CaCO}_{3}$ & 2 & 730 & -- & -- & -- & -- & 1,100 \\
\hline P16 & 06325000 & Silica, dissolved, in mg/L & 2 & 7.51 & -- & -- & -- & -- & 12.7 \\
\hline P16 & 06325000 & Sulfate, dissolved, in $\mathrm{mg} / \mathrm{L}$ & 2 & 1,240 & -- & -- & -- & -- & 1,990 \\
\hline P16 & 06325000 & Dissolved solids, calculated, in $\mathrm{mg} / \mathrm{L}$ & 2 & 2,060 & -- & -- & -- & -- & 3,300 \\
\hline P16 & 06325000 & Dissolved solids, tons/day & 2 & 42.1 & -- & -- & -- & -- & 72.5 \\
\hline P17 & 453209105201201 & Streamflow, in cubic feet per second & 3 & 0.01 & -- & -- & -- & -- & 85 \\
\hline P17 & 453209105201201 & Turbidity, nephelometric turbidity ratio units & 3 & 4.2 & -- & -- & -- & -- & 22 \\
\hline P17 & 453209105201201 & Dissolved oxygen, in mg/L & 3 & 5.8 & -- & -- & -- & -- & 11.1 \\
\hline P17 & 453209105201201 & Dissolved oxygen, percent saturation & 2 & 84 & -- & -- & -- & -- & 112 \\
\hline P17 & 453209105201201 & $\mathrm{pH}$, in standard units & 3 & 8.1 & -- & -- & -- & -- & 8.4 \\
\hline P17 & 453209105201201 & Specific conductance, at 25 degrees Celsius, in $\mu \mathrm{S} / \mathrm{cm}$ & 3 & 1,820 & -- & -- & -- & -- & 3,180 \\
\hline P17 & 453209105201201 & Temperature, water deg C & 3 & 25 & -- & -- & -- & -- & 32.5 \\
\hline P17 & 453209105201201 & Hardness, in $\mathrm{mg} / \mathrm{L}$ as $\mathrm{CaCO}_{3}$ & 3 & 600 & -- & -- & -- & -- & 830 \\
\hline P17 & 453209105201201 & Calcium, dissolved, in $\mathrm{mg} / \mathrm{L}$ & 3 & 136 & -- & -- & -- & -- & 189 \\
\hline P17 & 453209105201201 & Magnesium, dissolved, in $\mathrm{mg} / \mathrm{L}$ & 3 & 62.6 & -- & -- & -- & -- & 97.8 \\
\hline P17 & 453209105201201 & Potassium, dissolved, in $\mathrm{mg} / \mathrm{L}$ & 3 & 7.37 & -- & -- & -- & -- & 16.2 \\
\hline P17 & 453209105201201 & Sodium-adsorption ratio (unitless) & 3 & 3.7 & -- & -- & -- & -- & 7.1 \\
\hline P17 & 453209105201201 & Sodium, dissolved, in $\mathrm{mg} / \mathrm{L}$ & 3 & 206 & -- & -- & -- & -- & 466 \\
\hline P17 & 453209105201201 & Alkalinity, in $\mathrm{mg} / \mathrm{L}$ as $\mathrm{CaCO}_{3}$ & 3 & 201 & -- & -- & -- & -- & 289 \\
\hline P17 & 453209105201201 & Chloride, dissolved, in $\mathrm{mg} / \mathrm{L}$ & 3 & 76.1 & -- & -- & -- & -- & 128 \\
\hline P17 & 453209105201201 & Fluoride, dissolved, in $\mathrm{mg} / \mathrm{L}$ & 3 & 0.33 & -- & -- & -- & -- & 0.56 \\
\hline
\end{tabular}


Appendix. Summary statistics for water-quality constituents, Powder River structural basin, Wyoming and Montana, water years 2005-08.-Continued

[--, value cannot be calculated; $\mathrm{mg} / \mathrm{L}$, milligrams per liter; $\mu \mathrm{S} / \mathrm{cm}$, microsiemens per centimeter at 25 degrees Celsius; deg $\mathrm{C}$, degrees $\mathrm{Celsius}^{\mathrm{C}} \mathrm{CaCO}_{3}$, calcium carbonate; as $\mathrm{N}$, as nitrogen; as $\mathrm{P}$, as phosphorus; $\mu \mathrm{g} / \mathrm{L}$, micrograms per liter; $\mathrm{ng} / \mathrm{L}$, nanograms per liter; mm, millimeters; *, constituent statistics were estimated using a log-probability regression; **, too many non-detected constituent concentrations to estimate statistics with log-probablity regression]

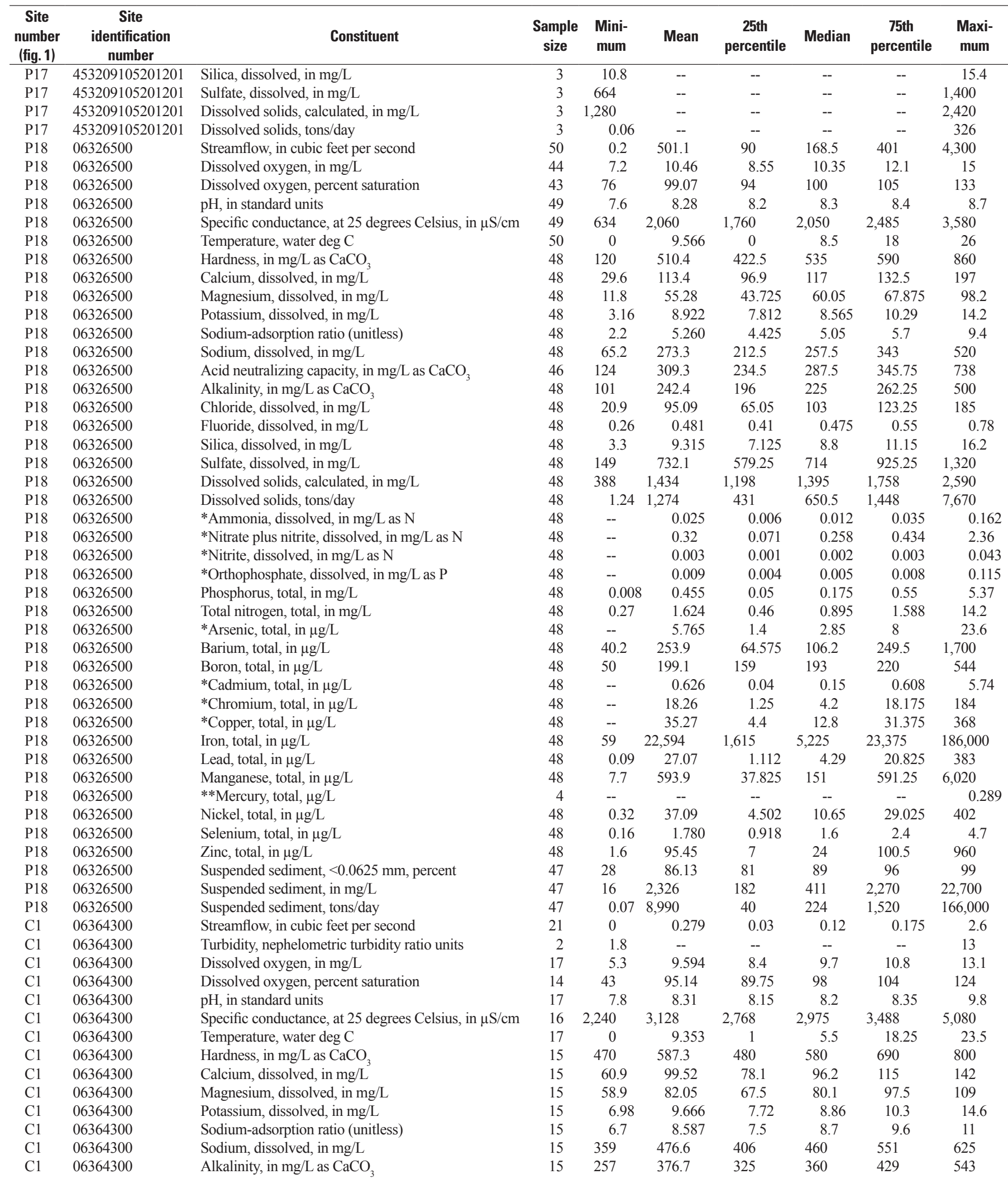


Appendix. Summary statistics for water-quality constituents, Powder River structural basin, Wyoming and Montana, water years 2005-08.-Continued

[--, value cannot be calculated; $\mathrm{mg} / \mathrm{L}$, milligrams per liter; $\mu \mathrm{S} / \mathrm{cm}$, microsiemens per centimeter at 25 degrees Celsius; deg $\mathrm{C}$, degrees Celsius; $\mathrm{CaCO}_{3}$, calcium carbonate; as $\mathrm{N}$, as nitrogen; as $\mathrm{P}$, as phosphorus; $\mu \mathrm{g} / \mathrm{L}$, micrograms per liter; ng/L, nanograms per liter; mm, millimeters; *, constituent statistics were estimated using a log-probability regression; **, too many non-detected constituent concentrations to estimate statistics with log-probablity regression]

\begin{tabular}{|c|c|c|c|c|c|c|c|c|c|}
\hline $\begin{array}{c}\text { Site } \\
\text { number } \\
\text { (fig. 1) }\end{array}$ & $\begin{array}{c}\text { Site } \\
\text { identification } \\
\text { number }\end{array}$ & Constituent & $\begin{array}{l}\text { Sample } \\
\text { size }\end{array}$ & $\begin{array}{l}\text { Mini- } \\
\text { mum }\end{array}$ & Mean & $\begin{array}{c}\text { 25th } \\
\text { percentile }\end{array}$ & Median & $\begin{array}{c}\text { 75th } \\
\text { percentile }\end{array}$ & $\begin{array}{l}\text { Maxi- } \\
\text { mum }\end{array}$ \\
\hline $\mathrm{C} 1$ & 06364300 & Chloride, dissolved, in $\mathrm{mg} / \mathrm{L}$ & 15 & 12.6 & 18.85 & 15.7 & 17.4 & 22.8 & 26.4 \\
\hline $\mathrm{C} 1$ & 06364300 & Fluoride, dissolved, in mg/L & 15 & 0.9 & 1.252 & 1.1 & 1.19 & 1.39 & 1.79 \\
\hline $\mathrm{C} 1$ & 06364300 & Sulfate, dissolved, in $\mathrm{mg} / \mathrm{L}$ & 15 & 890 & 1,205 & 983 & 1,180 & 1,290 & 1,800 \\
\hline $\mathrm{C} 1$ & 06364300 & Dissolved solids, calculated, in $\mathrm{mg} / \mathrm{L}$ & 15 & 1,580 & 2,122 & 1,790 & 2,080 & 2,380 & 2,920 \\
\hline $\mathrm{C} 1$ & 06364300 & Dissolved solids, tons/day & 15 & 0.13 & 1.132 & 0.45 & 0.9 & 1.38 & 4.79 \\
\hline $\mathrm{C} 1$ & 06364300 & Arsenic, dissolved, in $\mu \mathrm{g} / \mathrm{L}$ & 15 & 1.3 & 2.127 & 1.7 & 2 & 2.3 & 4.5 \\
\hline $\mathrm{C} 1$ & 06364300 & Arsenic, total, in $\mu \mathrm{g} / \mathrm{L}$ & 8 & 2.1 & 2.450 & 2.125 & 2.25 & 2.825 & 3.2 \\
\hline $\mathrm{C} 1$ & 06364300 & Barium, total, in $\mu \mathrm{g} / \mathrm{L}$ & 15 & 19.5 & 51.13 & 26.3 & 51.4 & 74.5 & 101 \\
\hline $\mathrm{C} 1$ & 06364300 & $* *$ Beryllium, total, in $\mu \mathrm{g} / \mathrm{L}$ & 15 & -- & -- & -- & -- & -- & $<0.12$ \\
\hline $\mathrm{C} 1$ & 06364300 & *Iron, dissolved, in $\mu \mathrm{g} / \mathrm{L}$ & 15 & -- & 28.91 & 12 & 28 & 31 & 117 \\
\hline $\mathrm{C} 1$ & 06364300 & Manganese, dissolved, in $\mu \mathrm{g} / \mathrm{L}$ & 15 & 47.2 & 150.8 & 72.9 & 118 & 166 & 515 \\
\hline $\mathrm{C} 2$ & 06364700 & $\mathrm{pH}$, in standard units & 23 & 7.1 & 7.55 & 7.3 & 7.6 & 7.7 & 8.1 \\
\hline $\mathrm{C} 2$ & 06364700 & Specific conductance, at 25 degrees Celsius, in $\mu \mathrm{S} / \mathrm{cm}$ & 23 & 1,820 & 2,994 & 2,910 & 3,090 & 3,130 & 3,200 \\
\hline $\mathrm{C} 2$ & 06364700 & Temperature, water deg C & 23 & 0.5 & 10.55 & 3.5 & 9.5 & 15 & 29.1 \\
\hline $\mathrm{C} 2$ & 06364700 & Hardness, in $\mathrm{mg} / \mathrm{L}$ as $\mathrm{CaCO}_{3}$ & 20 & 730 & 1,332 & 1,300 & 1,300 & 1,400 & 1,500 \\
\hline $\mathrm{C} 2$ & 06364700 & Calcium, dissolved, in $\mathrm{mg} / \mathrm{L}$ & 20 & 192 & 317.7 & 308.5 & 321.5 & 336.25 & 361 \\
\hline $\mathrm{C} 2$ & 06364700 & Magnesium, dissolved, in $\mathrm{mg} / \mathrm{L}$ & 20 & 61.1 & 132.2 & 132.25 & 136 & 138.75 & 151 \\
\hline $\mathrm{C} 2$ & 06364700 & Potassium, dissolved, in mg/L & 20 & 14.7 & 19.54 & 18.775 & 19.8 & 20.9 & 21.7 \\
\hline $\mathrm{C} 2$ & 06364700 & Sodium-adsorption ratio (unitless) & 20 & 1.9 & 3.050 & 2.825 & 3.15 & 3.275 & 3.7 \\
\hline $\mathrm{C} 2$ & 06364700 & Sodium, dissolved, in $\mathrm{mg} / \mathrm{L}$ & 20 & 121 & 257.2 & 239.5 & 268 & 282.75 & 308 \\
\hline $\mathrm{C} 2$ & 06364700 & Alkalinity, in $\mathrm{mg} / \mathrm{L}$ as $\mathrm{CaCO}_{3}$ & 20 & 320 & 388.9 & 353.5 & 396 & 417.5 & 438 \\
\hline $\mathrm{C} 2$ & 06364700 & Chloride, dissolved, in $\mathrm{mg} / \mathrm{L}$ & 20 & 7.62 & 20.39 & 19.725 & 21.05 & 22.45 & 23.3 \\
\hline $\mathrm{C} 2$ & 06364700 & Fluoride, dissolved, in $\mathrm{mg} / \mathrm{L}$ & 20 & 0.64 & 0.712 & 0.673 & 0.71 & 0.738 & 0.79 \\
\hline $\mathrm{C} 2$ & 06364700 & Silica, dissolved, in $\mathrm{mg} / \mathrm{L}$ & 20 & 15.3 & 19.09 & 17.6 & 18.75 & 20.95 & 24.4 \\
\hline $\mathrm{C} 2$ & 06364700 & Total nitrogen, total, in $\mathrm{mg} / \mathrm{L}$ & 12 & 0.24 & 0.379 & 0.29 & 0.325 & 0.447 & 0.7 \\
\hline $\mathrm{C} 2$ & 06364700 & Aluminum, total, in $\mu \mathrm{g} / \mathrm{L}$ & 20 & 11 & 38.40 & 15.25 & 39 & 55.75 & 119 \\
\hline $\mathrm{C} 2$ & 06364700 & Arsenic, dissolved, in $\mu \mathrm{g} / \mathrm{L}$ & 20 & 0.42 & 0.646 & 0.505 & 0.62 & 0.7 & 1.1 \\
\hline $\mathrm{C} 2$ & 06364700 & *Arsenic, total, in $\mu \mathrm{g} / \mathrm{L}$ & 12 & -- & 0.907 & 0.778 & 0.89 & 0.983 & 1.3 \\
\hline $\mathrm{C} 2$ & 06364700 & Barium, total, in $\mu \mathrm{g} / \mathrm{L}$ & 20 & 22.4 & 40.29 & 24.85 & 27.35 & 34 & 192 \\
\hline $\mathrm{C} 2$ & 06364700 & $* *$ Beryllium, total, in $\mu \mathrm{g} / \mathrm{L}$ & 20 & -- & -- & -- & -- & -- & $<0.18$ \\
\hline $\mathrm{C} 2$ & 06364700 & Iron, dissolved, in $\mu \mathrm{g} / \mathrm{L}$ & 20 & 14 & 431.6 & 25 & 207.5 & 592.5 & 1,700 \\
\hline $\mathrm{C} 2$ & 06364700 & Manganese, dissolved, in $\mu \mathrm{g} / \mathrm{L}$ & 20 & 402 & 1,735 & 1,045 & 1,545 & 2,100 & 4,850 \\
\hline $\mathrm{C} 2$ & 06364700 & $*$ Selenium, total, in $\mu \mathrm{g} / \mathrm{L}$ & 20 & -- & 0.741 & 0.135 & 0.22 & 1.475 & 2.4 \\
\hline $\mathrm{C} 3$ & 06365900 & Streamflow, in cubic feet per second & 50 & 0 & 0.103 & 0 & 0.01 & 0.04 & 4.3 \\
\hline $\mathrm{C} 3$ & 06365900 & Turbidity, nephelometric turbidity ratio units & 2 & 6 & -- & -- & -- & -- & 49 \\
\hline $\mathrm{C} 3$ & 06365900 & Dissolved oxygen, in $\mathrm{mg} / \mathrm{L}$ & 30 & 5.1 & 7.547 & 6.475 & 7.85 & 8.575 & 9.7 \\
\hline $\mathrm{C} 3$ & 06365900 & Dissolved oxygen, percent saturation & 26 & 41 & 70.96 & 63.75 & 71.5 & 80.25 & 95 \\
\hline $\mathrm{C} 3$ & 06365900 & $\mathrm{pH}$, in standard units & 31 & 7.2 & 7.69 & 7.5 & 7.8 & 7.9 & 8.1 \\
\hline $\mathrm{C} 3$ & 06365900 & Specific conductance, at 25 degrees Celsius, in $\mu \mathrm{S} / \mathrm{cm}$ & 29 & 263 & 3,814 & 3,275 & 3,870 & 4,185 & 6,560 \\
\hline $\mathrm{C} 3$ & 06365900 & Temperature, water deg C & 31 & 0 & 7.158 & 0 & 3.5 & 12.9 & 30.6 \\
\hline $\mathrm{C} 3$ & 06365900 & Hardness, in $\mathrm{mg} / \mathrm{L}$ as $\mathrm{CaCO}_{3}$ & 29 & 65 & 1,448 & 1,250 & 1,500 & 1,650 & 2,100 \\
\hline $\mathrm{C} 3$ & 06365900 & Calcium, dissolved, in $\mathrm{mg} / \mathrm{L}$ & 29 & 16.2 & 322.6 & 277 & 332 & 371.5 & 479 \\
\hline $\mathrm{C} 3$ & 06365900 & Magnesium, dissolved, in mg/L & 29 & 6.01 & 157.0 & 136.5 & 162 & 181.5 & 226 \\
\hline $\mathrm{C} 3$ & 06365900 & Potassium, dissolved, in $\mathrm{mg} / \mathrm{L}$ & 29 & 8.49 & 18.65 & 15 & 20 & 21.85 & 28.1 \\
\hline
\end{tabular}


Appendix. Summary statistics for water-quality constituents, Powder River structural basin, Wyoming and Montana, water years 2005-08. - Continued

[--, value cannot be calculated; $\mathrm{mg} / \mathrm{L}$, milligrams per liter; $\mu \mathrm{S} / \mathrm{cm}$, microsiemens per centimeter at 25 degrees Celsius; deg $\mathrm{C}$, degrees Celsius; $\mathrm{CaCO}_{3}$, calcium carbonate; as $\mathrm{N}$, as nitrogen; as $\mathrm{P}$, as phosphorus; $\mu \mathrm{g} / \mathrm{L}$, micrograms per liter; $\mathrm{ng} / \mathrm{L}$, nanograms per liter; mm, millimeters; *, constituent statistics were estimated using a log-probability regression; **, too many non-detected constituent concentrations to estimate statistics with log-probablity regression]

\begin{tabular}{|c|c|c|c|c|c|c|c|c|c|}
\hline $\begin{array}{l}\text { Site } \\
\text { number } \\
\text { (fig. 1) }\end{array}$ & $\begin{array}{c}\text { Site } \\
\text { identification } \\
\text { number }\end{array}$ & Constituent & $\begin{array}{c}\text { Sample } \\
\text { size }\end{array}$ & $\begin{array}{l}\text { Mini- } \\
\text { mum }\end{array}$ & Mean & $\begin{array}{c}\text { 25th } \\
\text { percentile }\end{array}$ & Median & $\begin{array}{c}\text { 75th } \\
\text { percentile }\end{array}$ & $\begin{array}{l}\text { Maxi- } \\
\text { mum }\end{array}$ \\
\hline $\mathrm{C} 3$ & 06365900 & Sodium-adsorption ratio (unitless) & 29 & 0.9 & 4.886 & 4.45 & 4.9 & 5.4 & 7.1 \\
\hline $\mathrm{C} 3$ & 06365900 & Sodium, dissolved, in mg/L & 29 & 17.1 & 435.5 & 372 & 438 & 502 & 732 \\
\hline $\mathrm{C} 3$ & 06365900 & Chloride, dissolved, in $\mathrm{mg} / \mathrm{L}^{3}$ & 29 & 3.28 & 27.17 & 26.15 & 28.3 & 31.05 & 34.4 \\
\hline $\mathrm{C} 3$ & 06365900 & Fluoride, dissolved, in mg/L & 29 & 0.18 & 0.632 & 0.59 & 0.64 & 0.69 & 0.86 \\
\hline $\mathrm{C} 3$ & 06365900 & Silica, dissolved, in $\mathrm{mg} / \mathrm{L}$ & 29 & 3.67 & 12.46 & 9.235 & 12.2 & 15.6 & 22.2 \\
\hline $\mathrm{C} 3$ & 06365900 & Dissolved solids, tons/day & 28 & 0.02 & 0.344 & 0.14 & 0.26 & 0.377 & 2.05 \\
\hline $\mathrm{C} 3$ & 06365900 & Dissolved solids, residue on evaporation, in $\mathrm{mg} / \mathrm{L}$ & 29 & 175 & 3,415 & 2,905 & 3,430 & 3,845 & 5,180 \\
\hline $\mathrm{C} 3$ & 06365900 & Aluminum, total, in $\mu \mathrm{g} / \mathrm{L}$ & 29 & 4 & 599.0 & 22 & 35 & 64.5 & 16,100 \\
\hline $\mathrm{C} 3$ & 06365900 & Arsenic, dissolved, in $\mu \mathrm{g} / \mathrm{L}$ & 29 & 0.45 & 0.729 & 0.525 & 0.59 & 0.95 & 1.4 \\
\hline $\mathrm{C} 3$ & 06365900 & $*$ Arsenic, total, in $\mu \mathrm{g} / \mathrm{L}$ & 20 & -- & 1.190 & 0.762 & 0.965 & 1.3 & 3.9 \\
\hline $\mathrm{C} 3$ & 06365900 & Barium, total, in $\mu \mathrm{g} / \mathrm{L}$ & 29 & 14.8 & 39.17 & 20.25 & 25.6 & 30.8 & 258 \\
\hline $\mathrm{C} 4$ & 06375600 & Turbidity, nephelometric turbidity ratio units & 3 & 19 & -- & -- & -- & -- & 42 \\
\hline $\mathrm{C} 4$ & 06375600 & Dissolved oxygen, in $\mathrm{mg} / \mathrm{L}$ & 14 & 3.4 & 7.371 & 5.475 & 7.7 & 9.325 & 10.8 \\
\hline $\mathrm{C} 4$ & 06375600 & Dissolved oxygen, percent saturation & 11 & 46 & 88.55 & 67 & 93 & 112 & 124 \\
\hline $\mathrm{C} 4$ & 06375600 & $\mathrm{pH}$, in standard units & 14 & 7.5 & 8.18 & 7.68 & 8.2 & 8.48 & 8.9 \\
\hline $\mathrm{C} 4$ & 06375600 & Specific conductance, at 25 degrees Celsius, in $\mu \mathrm{S} / \mathrm{cm}$ & 14 & 232 & 1,798 & 858.75 & 1,610 & 2,075 & 6,370 \\
\hline $\mathrm{C} 4$ & 06375600 & Temperature, water $\operatorname{deg} \mathrm{C}$ & 14 & 8 & 17.84 & 14.225 & 19 & 21.7 & 24.1 \\
\hline $\mathrm{C} 4$ & 06375600 & Hardness, in $\mathrm{mg} / \mathrm{L}$ as $\mathrm{CaCO}_{3}$ & 11 & 59 & 339.7 & 160 & 350 & 550 & 720 \\
\hline $\mathrm{C} 4$ & 06375600 & Calcium, dissolved, in $\mathrm{mg} / \mathrm{L}$ & 11 & 13.1 & 65.97 & 34.7 & 64.9 & 114 & 130 \\
\hline $\mathrm{C} 4$ & 06375600 & Magnesium, dissolved, in $\mathrm{mg} / \mathrm{L}$ & 11 & 6.47 & 42.51 & 16.6 & 42.8 & 63.8 & 94.9 \\
\hline $\mathrm{C} 4$ & 06375600 & Sodium-adsorption ratio (unitless) & 11 & 0.9 & 4.264 & 2 & 4.3 & 5.8 & 8.3 \\
\hline $\mathrm{C} 4$ & 06375600 & Sodium, dissolved, in mg/L & 11 & 15.2 & 195.7 & 57.3 & 183 & 277 & 508 \\
\hline $\mathrm{C} 4$ & 06375600 & Dissolved solids, residue on evaporation, in $\mathrm{mg} / \mathrm{L}$ & 11 & 145 & 1,046 & 394 & 987 & 1,380 & 2,460 \\
\hline $\mathrm{C} 4$ & 06375600 & **Ammonia, dissolved, in $\mathrm{mg} / \mathrm{L}$ as $\mathrm{N}$ & 9 & -- & -- & -- & -- & -- & 0.046 \\
\hline C5 & 06376300 & $\mathrm{pH}$, in standard units & 12 & 7.5 & 7.98 & 7.73 & 7.95 & 8.28 & 8.5 \\
\hline $\mathrm{C} 5$ & 06376300 & Specific conductance, at 25 degrees Celsius, in $\mu \mathrm{S} / \mathrm{cm}$ & 12 & 197 & 510.8 & 226.5 & 424 & 723.75 & 1,410 \\
\hline C5 & 06376300 & Temperature, water deg C & 12 & 0.5 & 16.79 & 15.55 & 17.35 & 22.15 & 24.4 \\
\hline $\mathrm{C} 5$ & 06376300 & Hardness, in $\mathrm{mg} / \mathrm{L}$ as $\mathrm{CaCO}_{3}$ & 10 & 40 & 113.1 & 47.75 & 104 & 162.5 & 240 \\
\hline $\mathrm{C} 5$ & 06376300 & Calcium, dissolved, in $\mathrm{mg} / \mathrm{L}$ & 10 & 8.9 & 26.39 & 11.225 & 24.2 & 37.35 & 58.5 \\
\hline C5 & 06376300 & Magnesium, dissolved, in $\mathrm{mg} / \mathrm{L}$ & 10 & 4.23 & 11.48 & 4.86 & 10.59 & 16.725 & 23.3 \\
\hline C5 & 06376300 & Potassium, dissolved, in $\mathrm{mg} / \mathrm{L}$ & 10 & 4.22 & 7.485 & 5.493 & 7.595 & 9.023 & 11.1 \\
\hline $\mathrm{C} 5$ & 06376300 & Sodium-adsorption ratio (unitless) & 10 & 0.9 & 1.720 & 1.1 & 1.75 & 2.3 & 2.4 \\
\hline $\mathrm{C} 5$ & 06376300 & Sodium, dissolved, in mg/L & 10 & 14.1 & 43.20 & 20.575 & 38.45 & 63.475 & 84.9 \\
\hline $\mathrm{C} 5$ & 06376300 & Alkalinity, in $\mathrm{mg} / \mathrm{L}$ as $\mathrm{CaCO}_{3}$ & 10 & 34 & 80.20 & 44.5 & 75.5 & 121.25 & 139 \\
\hline $\mathrm{C} 5$ & 06376300 & Chloride, dissolved, in $\mathrm{mg} / \mathrm{L}$ & 10 & 0.84 & 3.954 & 1.872 & 3.82 & 5.905 & 7.72 \\
\hline $\mathrm{C} 5$ & 06376300 & Fluoride, dissolved, in mg/L & 10 & 0.2 & 0.27 & 0.228 & 0.265 & 0.303 & 0.36 \\
\hline C5 & 06376300 & Silica, dissolved, in $\mathrm{mg} / \mathrm{L}$ & 10 & 0.68 & 5.217 & 3.257 & 5.455 & 6.948 & 8.74 \\
\hline $\mathrm{C} 5$ & 06376300 & Sulfate, dissolved, in $\mathrm{mg} / \mathrm{L}$ & 10 & 42.2 & 130.4 & 50.725 & 106.65 & 198.75 & 288 \\
\hline $\mathrm{C} 5$ & 06376300 & Dissolved solids, calculated, in $\mathrm{mg} / \mathrm{L}$ & 10 & 113 & 276.5 & 123.75 & 257 & 394.75 & 555 \\
\hline $\mathrm{C} 5$ & 06376300 & Dissolved solids, tons/day & 10 & 0.05 & 4.286 & 0.225 & 1.88 & 9.032 & 15.9 \\
\hline C5 & 06376300 & Dissolved solids, residue on evaporation, in $\mathrm{mg} / \mathrm{L}$ & 10 & 134 & 307.9 & 162.75 & 282.5 & 427.5 & 590 \\
\hline $\mathrm{C} 5$ & 06376300 & Aluminum, total, in $\mu \mathrm{g} / \mathrm{L}$ & 10 & 117 & 10,456 & 4,793 & 10,650 & 14,725 & 25,800 \\
\hline $\mathrm{C} 5$ & 06376300 & Arsenic, dissolved, in $\mu \mathrm{g} / \mathrm{L}$ & 10 & 0.49 & 0.781 & 0.623 & 0.76 & 0.903 & 1.2 \\
\hline $\mathrm{C} 5$ & 06376300 & Arsenic, total, in $\mu \mathrm{g} / \mathrm{L}$ & 8 & 0.93 & 3.741 & 2.525 & 3.9 & 4.575 & 6.4 \\
\hline
\end{tabular}


Appendix. Summary statistics for water-quality constituents, Powder River structural basin, Wyoming and Montana, water years 2005-08.-Continued

[--, value cannot be calculated; $\mathrm{mg} / \mathrm{L}$, milligrams per liter; $\mu \mathrm{S} / \mathrm{cm}$, microsiemens per centimeter at 25 degrees Celsius; deg $\mathrm{C}$, degrees Celsius; $\mathrm{CaCO}_{3}$, calcium carbonate; as $\mathrm{N}$, as nitrogen; as $\mathrm{P}$, as phosphorus; $\mu \mathrm{g} / \mathrm{L}$, micrograms per liter; ng/L, nanograms per liter; mm, millimeters; *, constituent statistics were estimated using a log-probability regression; **, too many non-detected constituent concentrations to estimate statistics with log-probablity regression]

\begin{tabular}{|c|c|c|c|c|c|c|c|c|c|}
\hline $\begin{array}{c}\text { Site } \\
\text { number } \\
\text { (fig. 1) } \\
\end{array}$ & $\begin{array}{c}\text { Site } \\
\begin{array}{c}\text { identification } \\
\text { number }\end{array} \\
\end{array}$ & Constituent & $\begin{array}{l}\text { Sample } \\
\text { size }\end{array}$ & $\begin{array}{l}\text { Mini- } \\
\text { mum }\end{array}$ & Mean & $\begin{array}{c}\text { 25th } \\
\text { percentile }\end{array}$ & Median & $\begin{array}{c}\text { 75th } \\
\text { percentile }\end{array}$ & $\begin{array}{l}\text { Maxi- } \\
\text { mum }\end{array}$ \\
\hline C5 & 06376300 & Barium, total, in $\mu \mathrm{g} / \mathrm{L}$ & 10 & 71.1 & 195.1 & 112.5 & 170 & 263.25 & 426 \\
\hline $\mathrm{C} 5$ & 06376300 & *Beryllium, total, in $\mu \mathrm{g} / \mathrm{L}$ & 10 & -- & 1.011 & 0.298 & 0.965 & 1.528 & 2.79 \\
\hline $\mathrm{C} 5$ & 06376300 & Manganese, dissolved, in $\mu \mathrm{g} / \mathrm{L}$ & 10 & 1.1 & 6.530 & 1.4 & 3 & 10.925 & 18.8 \\
\hline $\mathrm{C} 5$ & 06376300 & Selenium, total, in $\mu \mathrm{g} / \mathrm{L}$ & 10 & 0.57 & 1.213 & 0.8 & 1.05 & 1.825 & 2.1 \\
\hline C6 & 06386500 & Streamflow, in cubic feet per second & 44 & 0 & 5.893 & 0.012 & 0.12 & 1.275 & 121 \\
\hline C6 & 06386500 & Dissolved oxygen, percent saturation & 33 & 43 & 99.21 & 88.5 & 102 & 110.5 & 149 \\
\hline C6 & 06386500 & $\mathrm{pH}$, in standard units & 35 & 7.6 & 8.07 & 7.9 & 8.1 & 8.3 & 8.4 \\
\hline C6 & 06386500 & Specific conductance, at 25 degrees Celsius, in $\mu \mathrm{S} / \mathrm{cm}$ & 36 & 541 & 4,497 & 3,498 & 4,995 & 5,515 & 7,100 \\
\hline C6 & 06386500 & Temperature, water deg $\mathrm{C}$ & 36 & 0 & 13.75 & 2.125 & 13.5 & 23.85 & 30.5 \\
\hline C6 & 06386500 & Hardness, in $\mathrm{mg} / \mathrm{L}$ as $\mathrm{CaCO}_{3}$ & 34 & 120 & 1,015 & 772.5 & 1,100 & 1,300 & 1,700 \\
\hline C6 & 06386500 & Calcium, dissolved, in $\mathrm{mg} / \mathrm{L}^{3}$ & 34 & 31.1 & 236.7 & 178.5 & 247.5 & 307.75 & 407 \\
\hline C6 & 06386500 & Chloride, dissolved, in $\mathrm{mg} / \mathrm{L}^{3}$ & 34 & 5.27 & 88.75 & 65.6 & 97 & 110.75 & 148 \\
\hline C6 & 06386500 & Fluoride, dissolved, in mg/L & 34 & 0.3 & 0.564 & 0.53 & 0.57 & 0.623 & 0.72 \\
\hline C6 & 06386500 & Silica, dissolved, in $\mathrm{mg} / \mathrm{L}$ & 34 & 1.79 & 7.235 & 5.507 & 7.44 & 9.14 & 10.8 \\
\hline C6 & 06386500 & Sulfate, dissolved, in $\mathrm{mg} / \mathrm{L}$ & 34 & 150 & 2,193 & 1,593 & 2,485 & 2,815 & 3,490 \\
\hline C6 & 06386500 & Dissolved solids, calculated, in mg/L & 34 & 329 & 3,513 & 2,583 & 3,930 & 4,500 & 5,590 \\
\hline C6 & 06386500 & Dissolved solids, tons/day & 34 & 0.11 & 18.57 & 0.535 & 3.995 & 16.825 & 181 \\
\hline C6 & 06386500 & Dissolved solids, residue on evaporation, in $\mathrm{mg} / \mathrm{L}$ & 34 & 363 & 3,737 & 2,858 & 4,125 & 4,743 & 5,990 \\
\hline C6 & 06386500 & **Ammonia, dissolved, in $\mathrm{mg} / \mathrm{L}$ as $\mathrm{N}$ & 2 & -- & -- & -- & -- & -- & $<0.04$ \\
\hline C6 & 06386500 & **Nitrate plus nitrite, dissolved, in $\mathrm{mg} / \mathrm{L}$ as $\mathrm{N}$ & 2 & -- & -- & -- & -- & -- & $<0.06$ \\
\hline C6 & 06386500 & **Nitrite, dissolved, in $\mathrm{mg} / \mathrm{L}$ as $\mathrm{N}$ & 2 & -- & -- & -- & -- & -- & $<0.008$ \\
\hline C6 & 06386500 & **Orthophosphate, dissolved, in $\mathrm{mg} / \mathrm{L}$ as $\mathrm{P}$ & 2 & -- & -- & -- & -- & -- & $<0.02$ \\
\hline C6 & 06386500 & Total nitrogen, total, in $\mathrm{mg} / \mathrm{L}$ & 2 & 0.53 & -- & -- & -- & -- & 0.6 \\
\hline C6 & 06386500 & Aluminum, total, in $\mu \mathrm{g} / \mathrm{L}$ & 34 & 18 & 1,875 & 71.25 & 130.5 & 428 & 34,400 \\
\hline B1 & 06425720 & Turbidity, nephelometric turbidity ratio units & 2 & 4.3 & -- & -- & -- & -- & 5.7 \\
\hline B1 & 06425720 & Dissolved oxygen, in mg/L & 36 & 2.1 & 8.256 & 7.4 & 8.45 & 9.8 & 12.3 \\
\hline B1 & 06425720 & Dissolved oxygen, percent saturation & 34 & 17 & 82.03 & 68 & 87 & 97.5 & 136 \\
\hline B1 & 06425720 & $\mathrm{pH}$, in standard units & 37 & 7.5 & 8.08 & 7.8 & 8.1 & 8.3 & 8.8 \\
\hline B1 & 06425720 & Specific conductance, at 25 degrees Celsius, in $\mu \mathrm{S} / \mathrm{cm}$ & 37 & 1,760 & 3,068 & 2,630 & 2,920 & 3,335 & 6,210 \\
\hline B1 & 06425720 & Temperature, water deg C & 37 & 0 & 7.457 & 0 & 4.5 & 14.65 & 29.8 \\
\hline B1 & 06425720 & Hardness, in $\mathrm{mg} / \mathrm{L}$ as $\mathrm{CaCO}_{3}$ & 35 & 560 & 946.9 & 800 & 890 & 1,100 & 2,200 \\
\hline B1 & 06425720 & Calcium, dissolved, in $\mathrm{mg} / \mathrm{L}^{3}$ & 35 & 65.2 & 174.4 & 134 & 162 & 195 & 394 \\
\hline B1 & 06425720 & Magnesium, dissolved, in $\mathrm{mg} / \mathrm{L}$ & 35 & 67.4 & 124.5 & 107 & 120 & 137 & 300 \\
\hline B1 & 06425720 & Potassium, dissolved, in $\mathrm{mg} / \mathrm{L}$ & 29 & 8.47 & 13.66 & 11.3 & 12.6 & 15.3 & 26.7 \\
\hline B1 & 06425720 & Sodium-adsorption ratio (unitless) & 35 & 3.7 & 5.429 & 4.8 & 5.4 & 6.1 & 7.6 \\
\hline B1 & 06425720 & Sodium, dissolved, in mg/L & 35 & 200 & 385.2 & 319 & 372 & 445 & 825 \\
\hline B1 & 06425720 & Alkalinity, in $\mathrm{mg} / \mathrm{L}$ as $\mathrm{CaCO}_{3}$ & 29 & 90 & 295.5 & 227 & 282 & 314.5 & 778 \\
\hline B1 & 06425720 & Chloride, dissolved, in $\mathrm{mg} / \mathrm{L}$ & 29 & 10.2 & 23.51 & 17.5 & 20.4 & 28.5 & 62.1 \\
\hline B1 & 06425720 & Fluoride, dissolved, in $\mathrm{mg} / \mathrm{L}$ & 29 & 0.51 & 0.786 & 0.69 & 0.78 & 0.875 & 1.21 \\
\hline B1 & 06425720 & Silica, dissolved, in $\mathrm{mg} / \mathrm{L}$ & 29 & 0.37 & 2.489 & 1.245 & 1.83 & 3.07 & 9.68 \\
\hline B1 & 06425720 & Sulfate, dissolved, in $\mathrm{mg} / \mathrm{L}$ & 29 & 745 & 1,480 & 1,285 & 1,450 & 1,585 & 3,180 \\
\hline B1 & 06425720 & Dissolved solids, calculated, in $\mathrm{mg} / \mathrm{L}$ & 29 & 1,270 & 2,388 & 2,110 & 2,260 & 2,600 & 5,270 \\
\hline B1 & 06425720 & Dissolved solids, tons/day & 29 & 0.9 & 10.57 & 3.195 & 8.08 & 12.2 & 40.6 \\
\hline B1 & 06425720 & Dissolved solids, residue on evaporation, in $\mathrm{mg} / \mathrm{L}$ & 35 & 1,370 & 2,550 & 2,150 & 2,450 & 2,810 & 5,720 \\
\hline
\end{tabular}


Appendix. Summary statistics for water-quality constituents, Powder River structural basin, Wyoming and Montana, water years 2005-08.-Continued

[--, value cannot be calculated; $\mathrm{mg} / \mathrm{L}$, milligrams per liter; $\mu \mathrm{S} / \mathrm{cm}$, microsiemens per centimeter at 25 degrees Celsius; deg $\mathrm{C}$, degrees $\mathrm{Celsius}^{\mathrm{C}} \mathrm{CaCO}_{3}$, calcium carbonate; as $\mathrm{N}$, as nitrogen; as $\mathrm{P}$, as phosphorus; $\mu \mathrm{g} / \mathrm{L}$, micrograms per liter; $\mathrm{ng} / \mathrm{L}$, nanograms per liter; mm, millimeters; *, constituent statistics were estimated using a log-probability regression; **, too many non-detected constituent concentrations to estimate statistics with log-probablity regression]

\begin{tabular}{|c|c|c|c|c|c|c|c|c|c|}
\hline $\begin{array}{c}\text { Site } \\
\text { number } \\
\text { (fig. 1) }\end{array}$ & $\begin{array}{c}\text { Site } \\
\text { identification } \\
\text { number }\end{array}$ & Constituent & $\begin{array}{l}\text { Sample } \\
\text { size }\end{array}$ & $\begin{array}{c}\text { Mini- } \\
\text { mum }\end{array}$ & Mean & $\begin{array}{c}\text { 25th } \\
\text { percentile }\end{array}$ & Median & $\begin{array}{c}\text { 75th } \\
\text { percentile }\end{array}$ & $\begin{array}{l}\text { Maxi- } \\
\text { mum }\end{array}$ \\
\hline B1 & 06425720 & Aluminum, total, in $\mu \mathrm{g} / \mathrm{L}$ & 29 & 3 & 165.6 & 66.5 & 98 & 155 & 1,820 \\
\hline B1 & 06425720 & Arsenic, dissolved, in $\mu \mathrm{g} / \mathrm{L}$ & 29 & 0.46 & 0.955 & 0.705 & 0.81 & 1.045 & 2 \\
\hline B1 & 06425720 & Barium, total, in $\mu \mathrm{g} / \mathrm{L}$ & 29 & 20 & 37.61 & 29.8 & 35.1 & 42.95 & 81.5 \\
\hline B1 & 06425720 & $* *$ Beryllium, total, in $\mu \mathrm{g} / \mathrm{L}$ & 29 & -- & -- & -- & -- & -- & 0.15 \\
\hline B1 & 06425720 & Iron, dissolved, in $\mu \mathrm{g} / \mathrm{L}$ & 29 & 15 & 56.48 & 26 & 47 & 81 & 185 \\
\hline B2 & 06425900 & Streamflow, in cubic feet per second & 51 & 0 & 0.541 & 0 & 0 & 0.2 & 4.8 \\
\hline B2 & 06425900 & Turbidity, nephelometric turbidity ratio units & 2 & 21 & -- & -- & -- & -- & 160 \\
\hline B2 & 06425900 & Dissolved oxygen, in $\mathrm{mg} / \mathrm{L}$ & 20 & 4.7 & 9.505 & 8.2 & 9.85 & 11.125 & 12.4 \\
\hline B2 & 06425900 & Dissolved oxygen, percent saturation & 19 & 58 & 93.16 & 86 & 94 & 101 & 137 \\
\hline B2 & 06425900 & $\mathrm{pH}$, in standard units & 21 & 7.8 & 8.19 & 8 & 8.2 & 8.3 & 8.6 \\
\hline B2 & 06425900 & Specific conductance, at 25 degrees Celsius, in $\mu \mathrm{S} / \mathrm{cm}$ & 21 & 1,100 & 2,865 & 2,155 & 2,480 & 3,315 & 5,370 \\
\hline B2 & 06425900 & Sodium-adsorption ratio (unitless) & 19 & 2.3 & 4.279 & 3.5 & 4.4 & 4.9 & 6.4 \\
\hline B2 & 06425900 & Sodium, dissolved, in mg/L & 19 & 110 & 313.8 & 218 & 272 & 366 & 640 \\
\hline B2 & 06425900 & Alkalinity, in $\mathrm{mg} / \mathrm{L}$ as $\mathrm{CaCO}_{3}$ & 19 & 161 & 303.9 & 214 & 292 & 340 & 778 \\
\hline B2 & 06425900 & Chloride, dissolved, in $\mathrm{mg} / \mathrm{L}$ & 19 & 10.9 & 46.50 & 29.7 & 45.2 & 63.2 & 81.5 \\
\hline B2 & 06425900 & Fluoride, dissolved, in mg/L & 19 & 0.53 & 0.943 & 0.78 & 0.86 & 1.1 & 1.84 \\
\hline B2 & 06425900 & Silica, dissolved, in mg/L & 19 & 0.15 & 3.798 & 0.9 & 3.2 & 6.14 & 11 \\
\hline B2 & 06425900 & Sulfate, dissolved, in $\mathrm{mg} / \mathrm{L}$ & 19 & 384 & 1,309 & 810 & 1,110 & 1,580 & 3,210 \\
\hline B2 & 06425900 & Dissolved solids, calculated, in mg/L & 19 & 730 & 2,184 & 1,520 & 1,820 & 2,510 & 4,780 \\
\hline B2 & 06425900 & Dissolved solids, tons/day & 19 & 0.28 & 5.603 & 0.93 & 2.06 & 10.1 & 18.8 \\
\hline B2 & 06425900 & Dissolved solids, residue on evaporation, in $\mathrm{mg} / \mathrm{L}$ & 19 & 761 & 2,394 & 1,640 & 2,030 & 2,840 & 5,280 \\
\hline B2 & 06425900 & *Ammonia, dissolved, in $\mathrm{mg} / \mathrm{L}$ as $\mathrm{N}$ & 13 & -- & 0.023 & 0.012 & 0.017 & 0.025 & 0.067 \\
\hline B2 & 06425900 & ${ }^{* *}$ Nitrate plus nitrite, dissolved, in $\mathrm{mg} / \mathrm{L}$ as $\mathrm{N}$ & 13 & -- & -- & -- & -- & -- & $<0.06$ \\
\hline B2 & 06425900 & **Nitrite, dissolved, in $\mathrm{mg} / \mathrm{L}$ as $\mathrm{N}$ & 13 & -- & -- & -- & -- & -- & $<0.002$ \\
\hline B2 & 06425900 & Manganese, dissolved, in $\mu \mathrm{g} / \mathrm{L}$ & 19 & 6.1 & 76.89 & 15 & 55.9 & 109 & 258 \\
\hline B2 & 06425900 & Selenium, total, in $\mu \mathrm{g} / \mathrm{L}$ & 19 & 0.39 & 2.245 & 0.56 & 1.7 & 2.5 & 11.6 \\
\hline
\end{tabular}

Publishing support provided by:

Denver Publishing Service Center, Denver, Colorado

Manuscript approved for publication, June 9, 2010

For more information concerning this publication, contact: Center Director, USGS Wyoming Water Science Center 2617 E. Lincolnway, Suite B.

Cheyenne, WY 82001

(307) 778-2931

Or visit the Wyoming Water Science Center Web site at: http://wy.water.usgs.gov/index.htm/

This publication is available online at http://pubs.usgs.gov/sir/2010/5124 

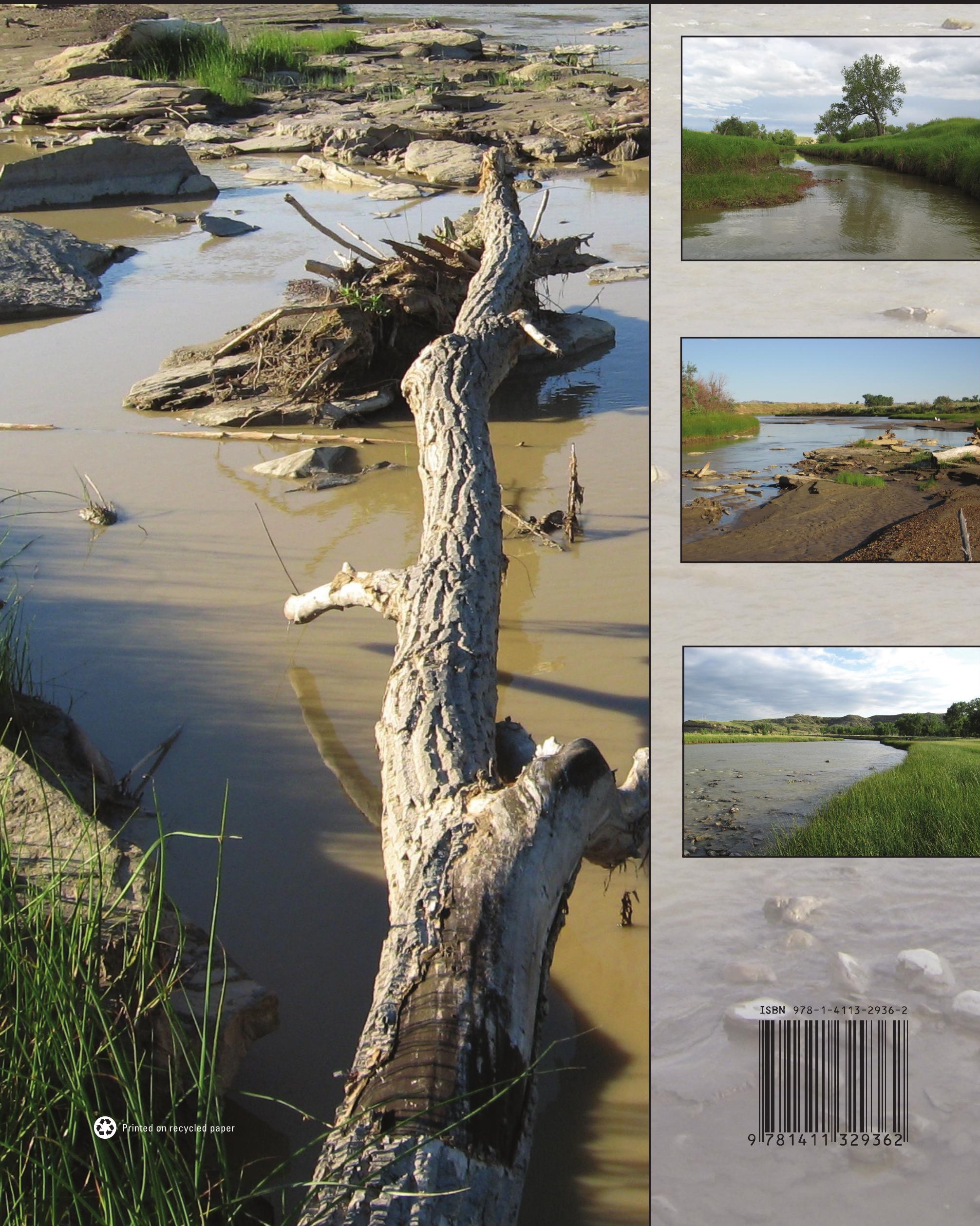

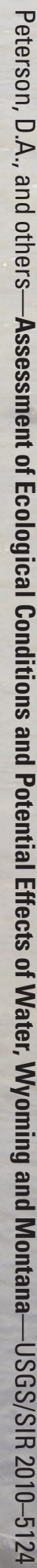
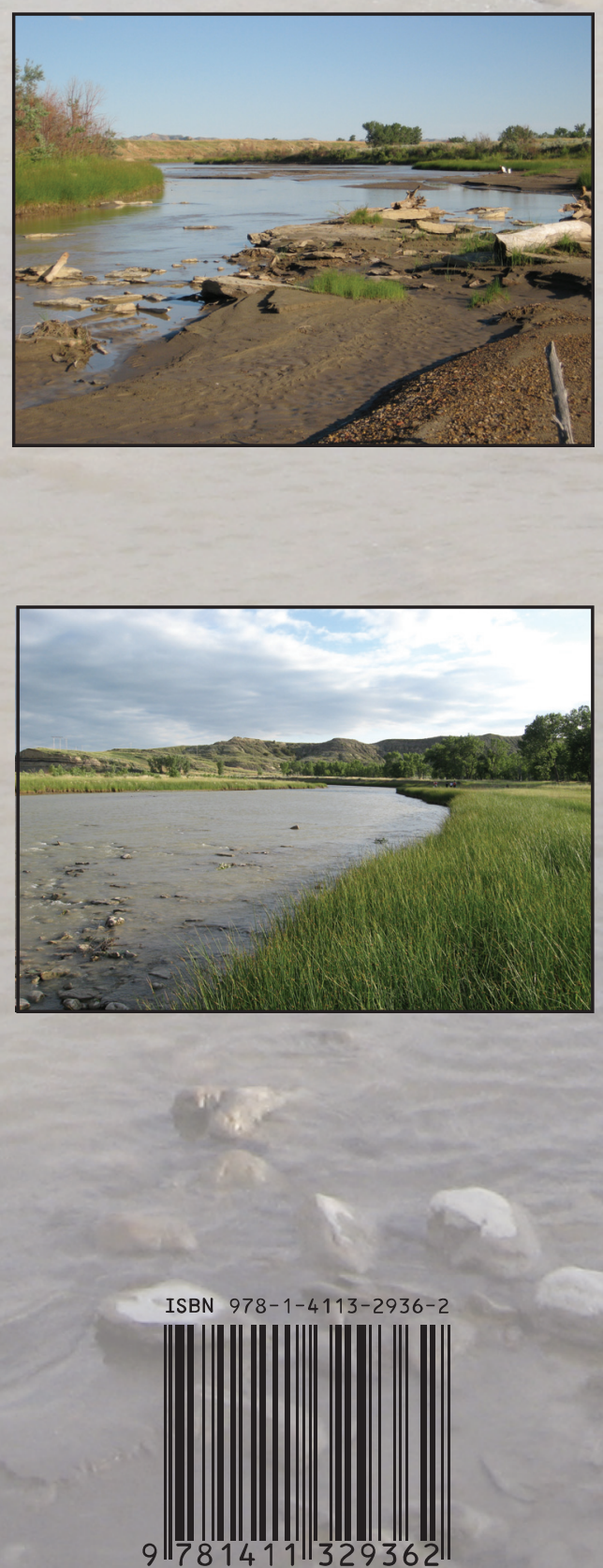